Portland State University

PDXScholar

2-11-1994

\title{
Laser Resonators Using Tiered Fresnel Mirrors
}

Bruce Dale Ulrich

Portland State University

Follow this and additional works at: https://pdxscholar.library.pdx.edu/open_access_etds

Part of the Electrical and Computer Engineering Commons Let us know how access to this document benefits you.

\section{Recommended Citation}

Ulrich, Bruce Dale, "Laser Resonators Using Tiered Fresnel Mirrors" (1994). Dissertations and Theses. Paper 4878.

https://doi.org/10.15760/etd.6754

This Thesis is brought to you for free and open access. It has been accepted for inclusion in Dissertations and Theses by an authorized administrator of PDXScholar. Please contact us if we can make this document more accessible: pdxscholar@pdx.edu. 


\section{THESIS APPROVAL}

The abstract and thesis of Bruce Dale Ulrich for the Master of Science in Electrical

Engineering were presented February 11, 1994 and accepted by the thesis committee and the department.

COMMITTEE APPROVALS:

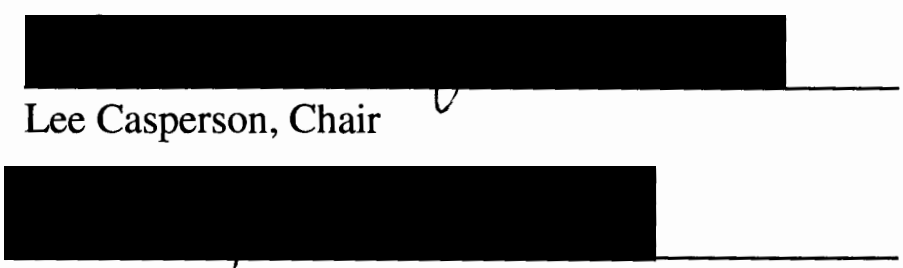

Branimir Pejcinovic

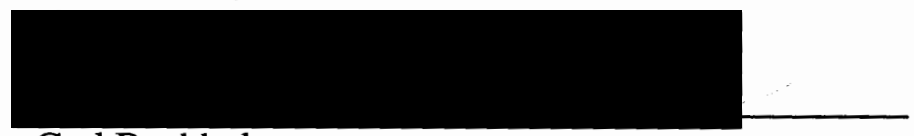

Carl Bachhuber

Representative of the Office of Graduate Studies

DEPARTMENT APPROVAL:

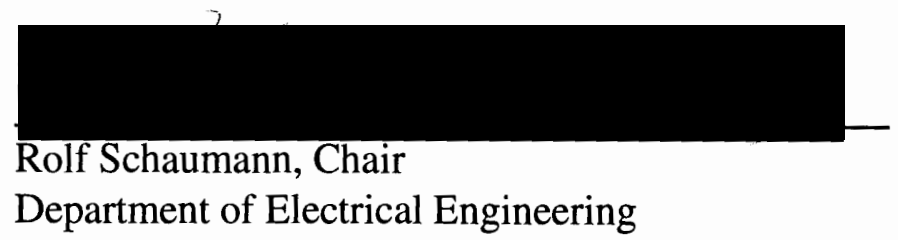

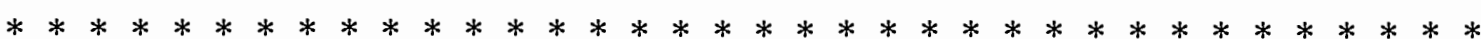




\begin{abstract}
An abstract of the thesis of Bruce Dale Ulrich for the Master of Science in Electrical Engineering presented February 11, 1994.
\end{abstract}

Title: Laser Resonators Using Tiered Fresnel Mirrors.

A reflective Tiered Fresnel Zone Plate, herein called a Tiered Fresnel Mirror TFM, with a focal length on the order of a meter is studied for use as the mirror(s) in a Fabry-Perot interferometer type of laser. The relative phase transition within the individual zones (ideally smooth from zero to pi ) is stair-stepped or tiered in the longitudinal direction of the mirror. Within an individual zone the step height is constrained to a constant whereas the width of the tiers are monotonically decreased when traversing radially outward so that the overall profile follows the ideal smooth curve. The effectiveness of the number of tiers per zone, measured by the loss per pass or round-trip, varies from a Plane Mirror (zero tiers per zone) to a Spherical Mirror (an infinite number of zones per tier).

The Fox and $\mathrm{Li}$ iterative method of determining the E-Field as the beam propagates back and forth is applied to an empty cavity resonator to determine the diffraction loss. A computer program is written to investigate the diffraction loss of various mirror configurations. The performance of the TFM is found to be not as efficient as the Spherical Mirror (the number of tiers per zone is shown to be a major variable) but may be tolerable under applications of a moderately high gain laser 
medium. The Gaussian Fundamental mode is easier to maintain since the higher order modes have a higher loss per round trip.

The manufacture of the TFM can be incorporated easily into an IC process thereby making the cost of the novel mirror relatively cheap when produced in quantities. A major cost variable is again the number of tiers per zone which is proportional to the number of processing steps. The TFM's performance with respect to the etch depth of the steps in the mirror's stair-stepped profile is simulated and found to be a very doable etch with the current plasma etch technology. 


\title{
LASER RESONATORS
}

\section{USING TIERED FRESNEL MIRRORS}

\author{
by \\ BRUCE DALE ULRICH
}

A thesis submitted in partial fulfillment of the requirements for the degree of

MASTER OF SCIENCE

in

ELECTRICAL ENGINEERING

Portland State University

1994 
TABLE OF CONTENTS

PAGE

LIST OF TABLES $\ldots \ldots \ldots \ldots \ldots \ldots \ldots \ldots \ldots$

LIST OF FIGURES . . . . . . . . . . . . . . . . . . . vi

\section{CHAPTER}

I INTRODUCTION $\ldots \ldots \ldots \ldots \ldots \ldots$

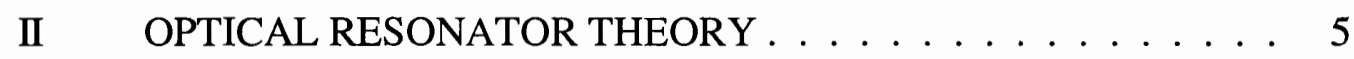

Matrix Optics . . . . . . . . . . . . . 5 5

Development of the Ray-Transfer Matrix, $\mathbf{T}$

The Stable Spherical Mirror Resonator . . . . . . . . . . . . 9

The Unbounded Lens Waveguide Method

The Self-Consistent Method

The Paraxial Wave Equation

Optical Resonator Algebra . . . . . . . . . . . . . . . 17

The Symmetric Mirror Resonator

Higher-Order Transverse Modes . . . . . . . . . . . . 22

Hermite-Gaussian Modes

Laguerre-Gaussian Modes

III RESONATOR MIRRORS . . . . . . . . . . . . . . . . 27

The Spherical Mirror . . . . . . . . . . . . . . 27

The Fresnel Mirror . . . . . . . . . . . . . . . . . . . . 29

The Fresnel Number N

The Tiered Fresnel Mirror . . . . . . . . . . . . . . . . 33 
Diffraction Losses . . . . . . . . . . . . . . . 33

The Fox and Li Method

IV COMPUTER SIMULATIONS . . . . . . . . . . . . . 42

V INTEGRATED CIRCUITS PROCESSING . . . . . . . . . . 62

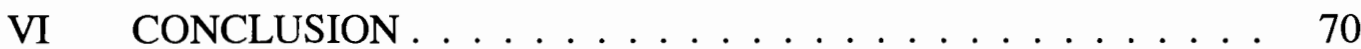

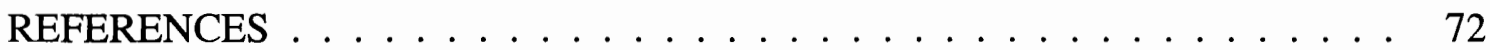

APPENDICES

A LISTING OF COMPUTER PROGRAM

"RESONATE VERSION $1.0 " \ldots \ldots 74$

B CROSS-REFERENCE MAP FOR COMPUTER

PROGRAM "RESONATE VERSION 1.0" . . . . . . . . . 142 


\section{LIST OF TABLES}

TABLE

PAGE

I Fractional Power Loss in a Symmetric Resonator . . . . . . . . 38

II Fractional Power Loss in a Non-Symmetric Resonator . . . . . . 40

III Simulated Tier Heights with Its Affect on Loss Per Pass

for a Symmetric Confocal Resonator . . . . . . . . . . . . 69 


\section{LIST OF FIGURES}

FIGURE

PAGE

1. Various Types of Zone Plates and Lenses . . . . . . . . . 2

2. Ray Propagation through a Homogeneous Dielectric of Length d . . 6

3. Ray Propagation through a "Thin" Lens . . . . . . . . . . 7

4. A Ray being reflected on a Spherical Mirror . . . . . . . . . 8

5. The Cascading of Ray-Transfer Matrices . . . . . . . . . . . . 9

6. An Empty Laser Cavity with Its Equivalent

Biperiodic Lens Sequence . . . . . . . . . . . . 10

7. The Beam Confinement (or Stability) Diagram

for Optical Resonators . . . . . . . . . . . . . . . 13

8. A Progressive Selection of Symmetric Mirror Curvatures

taken from Figure $7 \ldots \ldots \ldots$

9. The Confocal Resonator where $\mathrm{R}_{1}=\mathrm{R}_{2}=\mathrm{d} \ldots \ldots \ldots \ldots$

10. The Concentric Resonator where $\mathrm{R}_{1}=\mathrm{R}_{2}=\mathrm{d} / 2 \ldots \ldots \ldots$

11. The Plane-Parallel Resonator where $\mathrm{R}_{1}=\mathrm{R}_{2}=\infty \ldots \ldots \ldots 21$

12. The $(\pi / \lambda \mathrm{d})^{1 / 2}$ Scaled Beam Radius at the Waist, $\mathrm{w}_{0}$ and at the

Mirrors, $\mathrm{w}_{1}=\mathrm{w}_{2}$, for a Stable Symmetric Resonator as a Function of $d / R \ldots \ldots \ldots \ldots$

13. The $(\pi / \lambda \mathrm{d})^{1 / 2}$ Scaled Beam Radius at the Waist, $\mathrm{w}_{0}=\mathrm{w}_{1}$ at the

Plane Mirror and at the Concave Mirror, $\mathrm{w}_{2}$, for a

Stable Half Symmetric Resonator as a Function of $d / R \quad \ldots \quad 23$ 
14. A 3d Cross-Sectional View of: a) the Spherical Mirror, b) the Fresnel Mirror, and c) the Tiered(5) Fresnel Mirror having 5 Tiers Per Zone . . . . . . . . . . . . 28

15. Critical Points of the Spherical Mirror . . . . . . . . . . . 29

16. A Cross-Section of a Fresnel Mirror . . . . . . . . . . . 30

17. Critical Points of the Fresnel Mirror. Shown is a Radial

Plot of a Fresnel Mirror whose Size Spans the First

Three Fresnel Zones . . . . . . . . . . . . . . . . . . 31

18. A Circular Aperture with a Transmitted Plane Wave

Divided into Annular Rings. Each Ring is a

Fresnel Zone with N Equalling the Fresnel number . . . . 32

19. Critical Points of the Tiered Fresnel Mirror.

The Dotted Lines are the Edge Boundaries of the Tiers.

Each Tier Spans an Equal Phase . . . . . . . . . . . 34

20. The First Three Laguerre-Gaussian $\mathrm{TEM}_{\mathrm{p} 0}$ Modes vs.

the Waist Radius. The Modes Extend More Radially

with Increasing Mode Index p . . . . . . . . . . 35

21. Geometry of a Symmetric Resonator . . . . . . . . . . . . . 42

22. An Example Output of a Computer Simulation of a Tiered(5)

Fresnel Mirror Paired with a Spherical Mirror . . . . . . . . 44

23. The Difference Between the Theoretical and Simulated Values

of Loss Per Transit vs. the Fresnel Number of a

Symmetric Confocal Resonator $(\mathrm{d} / \mathrm{R}=1)$ and

another Symmetric Resonator where $\mathrm{d} / \mathrm{R}=1.5 \ldots 50$ 
24. The Mirror Radius Varies in a Symmetric Confocal Resonator in Figures 24 and 25. The top has $r=w$ and the bottom has $r=1.2 \mathrm{w} \ldots \ldots \ldots 52$

25. The Mirror Radius Varies in a Symmetric Confocal Resonator in Figures 24 and 25. The top has $r=1.5 \mathrm{w}$ and the bottom has $r=2 w \ldots \ldots \ldots \ldots$

26. A 25\% (Top) and a 50\% (Bottom) Completed Run of a Symmetric Resonator $(\mathrm{d} / \mathrm{R}=1.5)$.

Compare with Figure $27 \ldots \ldots$. . . . . . . . . . 54

27. A 75\% (Top) and a 100\% (Bottom) Completed Run of a Symmetric Resonator $(\mathrm{d} / \mathrm{R}=1.5)$. Compare with Figure $26 \ldots \ldots \ldots \ldots$

28. A Comparison of Mirror Types of a Symmetric Confocal

Resonator. Shown is a Spherical Type (top) and a Fresnel Type (Bottom) $\ldots \ldots \ldots \ldots$

29. A Tiered(15) Fresnel (Top) and a Tiered(5) Fresnel (Bottom)

Mirror Types of a Symmetric Confocal Resonator.

See Figure $28 \ldots \ldots \ldots$

30. A Comparison of Mirror Types of a $d / R=1.5$ Symmetric

Resonator. Shown is a Spherical Type (Top) and a Fresnel Type (Bottom) . . . . . . . . . . . . . . . 59

31. A Tiered(15) Fresnel (Top) and a Tiered(5) Fresnel (Bottom)

Mirror Types of a $d / R=1.5$ Symmetric Resonator.

See Figure $30 \ldots \ldots \ldots$

32. Mirror Performances of a $d / R=1$ and a $d / R=1.5$

Symmetric Resonator . . . . . . . . . . . . . . . . . 61 
33. A Radial Cross-Sectional View of a Fully Reflective Tiered(5) Fresnel Mirror with a Partially Transmitting

Plane Mirror. Note: This is a Half-Symmetric Resonator .

34. A Radial Cross-Sectional View of a Fully Reflective and a Partially Transmitting Tiered(5) Fresnel Mirrors . . . 64

35. A Zoom-In of the Bottom Mirror in Figure 34. The Particular Arrangement and Thicknesses of the Oxide and Nitride Film Stacks Convert a Curved Wavefront into a Planar Wavefront . . . . . . . . . . . . . . 65

36. A Process Sequence, from Start to Finish (Top to Bottom), of Radial Cross-Sections of the Tier Formation. A Reflective Type Tiered(5) Fresnel Mirror is Shown . . . . . 66

37. An Eight Inch Wafer Containing 185 Tiered(15) Fresnel Mirrors . 67

38. A Zoom-In of a Section of Figure 37. Notice There are 23 Tiers in this Three-Zone Tiered(15) Fresnel Mirror . . . 68 


\section{CHAPTER I}

\section{INTRODUCTION}

The Fresnel Zone Plate is a planar optical element with lens-like properties that can focus electromagnetic waves[1]. It is a transmission device, the electromagnetic waves transmit through it, focusing by diffraction and interference rather than by refraction.

There are various types of Fresnel Zone Plates as illustrated in Figure 1. In section $\mathrm{A}$ of the figure an amplitude type is shown where each alternate concentric ring is fully opaque (or non-transmitting) while the other rings are fully transmitting. The efficiency is not very good since close to $50 \%$ of the light is lost at the onset in front of the zone plate. In section B the Planar Lens is shown with two (or more) dielectrics being used in alternate concentric rings. The ideal is to have a relative phase difference of onehalf of a wavelength between adjacent rings. This is accomplished by the different dielectric constants of the materials. A proper choice of parameters results in a planar zone plate of constant thickness. In section $\mathrm{C}$ is a Phase-reversing Zone Plate. This is essentially the same concept as the Planar Lens Zone Plate of section B yet planarity is of no concern. In section D a Quarter-period Zone Plate is shown. Here each concentric ring is phase corrected in a stair-stepped or tiered manner. The correction spans from zero to pi radians of an optical cycle, that is a wavelength, and the zero is at the inner radius and the pi is at the outer radius of each ring. This particular zone plate is stairstepped with four tiers per ring, thus it is called a Quarter-period Zone Plate. In section E a Fresnel Lens is shown which is similar to the Quarter-period Zone Plate yet it has an infinite number of tiers within each ring making the profile a smooth transition from the 
inner to the outer part of each ring. This makes the phase perfectly corrected across each ring. The efficiency is nearly equal to the Simple Lens as is shown in section F of Figure 1.

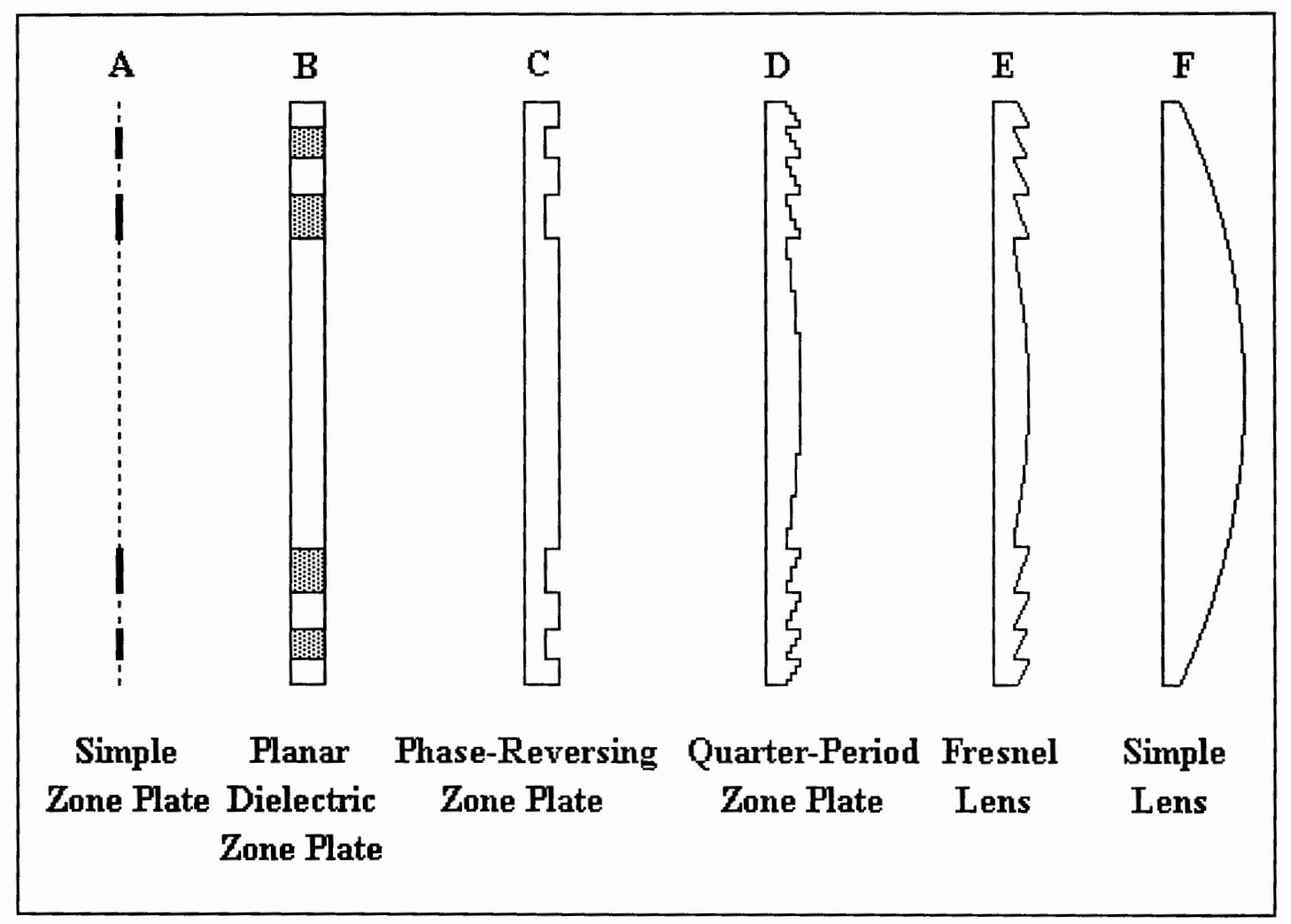

Figure 1. Various types of Zone Plates and Lenses.

Phase-correcting Fresnel Zone Plates are of particular interest since this principle is applied to a reflective type of zone plate described herein[2]. Micro-Fresnel Lenses with small apertures and large numerical apertures are required components in various optical systems such as pickup lenses in laser-disk players and coupler lenses in optical elements of optical communication systems. These have been made by laser and electron beam lithography[3,4]. A Fresnel Lens has been produced in an Integrated Circuits process on an oxidized silicon substrate using a silicon nitride waveguide[5]. A good review of reflection zone plates can be found in an article by Garrett and Wiltse[6]. 
Aspherical mirrors that are used in an optical resonator have been analyzed such that a large fundamental-mode beam width might be produced with a large transverse-mode discrimination[7].

The heart of this thesis is the study of a novel mirror called a Tiered Fresnel Mirror. It is used as the mirror component in an Optical Resonator. Generally, the mirrors at each end in a common laser system, that being basically a spherical mirror Fabry-Perot interferometer, are either both Spherical Mirrors or a combination of a Spherical and a Plane Mirror. Replacement of the Spherical Mirror with the Tiered Fresnel Mirror will be shown to be viable. Thus comparison of the Spherical Mirror to the Tiered Fresnel Mirror will be of utmost importance.

There already is the term "Fresnel Mirrors" used to describe two Plane Mirrors inclined at an angle to each other that produce an interference pattern [8]. No reference or use of these "Fresnel Mirrors" are contained in this thesis (only the terms "Fresnel Mirror" and "Tiered Fresnel Mirror" are used to identify another type of mirror). The Fresnel Mirror and the Tiered Fresnel Mirror will be defined in Chapter III and use of these terms will pertain to the definition given therein.

We will begin with a background discussion of the theory of Optical Resonators in Chapter II. This is a backbone chapter required for any study on Optical Resonators. Ray-Transfer Matrices are developed and the Stable Spherical Resonator is defined. Some important Symmetric Mirror Resonators are discussed. Finally, TEM Resonator Modes are defined.

Chapter III defines the Spherical Mirror, the Fresnel Mirror, and the Tiered Fresnel Mirror. The Fresnel Number N, an important number involved with the mirror loss due to diffraction, is discussed. Diffraction loss is also discussed and is the Figure of Merit for the performance of any mirror. Finally, the Fox and Li Method is used to determine the loss per pass in a Symmetric Resonator or the loss per round-trip in a Non- 
Symmetric Resonator. This method uses the Huygens-Fresnel Diffraction Integral via an iterated means to an end, namely the Diffraction Loss.

A computer program was written to perform the Fox and Li Method for many different resonator configurations. These computer simulations are contained in Chapter IV. In this chapter the comparison of the performance is made between the Spherical Mirror and : 1) the Fresnel Mirror; and 2) the Tiered Fresnel Mirror.

Integrated Circuits Processing is the key to the afford ability of the Tiered Fresnel Mirror. Chapter $\mathrm{V}$ is devoted to the discussion of incorporating the Tiered Fresnel Mirror instead of the Solid State Chip into an Integrated Circuits Process along with its benefits.

Chapter V is the conclusion chapter. The novel Tiered Fresnel Mirror will be deemed viable and affordable when manufactured with an Integrated Circuits Process.

A listing of the computer program "RESONATE ver 1.0" in included in Appendix A along with a cross-reference map of all the variables used in Appendix B for those interested in further work involved in diffraction integrals and/or the Fox and Li Method.

Finally, an appreciation goes to Dr. Lee Casperson for some helpful discussions, and his kindness and patience was warmly appreciated. 


\section{CHAPTER II}

\section{OPTICAL RESONATOR THEORY}

\section{MATRIX OPTICS}

Ray optics or geometrical optics is the simplest model of light propagation. This model applies when an optical system's components are much larger than the wavelength of light.

A ray can be thought of as the path that light takes at the center of a slowly diverging electromagnetic beam of small lateral extent compared to the optical components in an optical system. A ray that travels in a slight inclination to the optical axis is called a paraxial ray.

Ray optics deals with the location and direction of light rays and the redirection by an optical component. It is well known that, in an optical system, paraxial ray propagation can be characterized by a $2 \times 2$ matrix called the ray-transfer matrix $[9,10,11]$.

Matrix optics is a formal method of applying the ray-transfer matrix to characterize a paraxial optical system. Each component of this system has its own raytransfer matrix. When tracing the ray through this system, the ray-transfer matrix of the system is the product of the individual component's matrix. The output ray's location and direction can be found with relative ease, even in complex optical systems, in the paraxial approximation.

Development of the Ray-Transfer Matrix, $\mathbf{T}$. 
We will develop ray-transfer matrices for only three special optical components: the Homogeneous Dielectric; the "Thin" Lens; and the Spherical Mirror. The first and last elements are specifically used in the simple Spherical Mirror Resonator.

We begin by considering only paraxial rays in an optical system where the slope of the ray r' equals the angle measured with respect to the optical axis.

Homogeneous Dielectric Ray Propagation. Figure 2 shows the initial position $r_{\text {in }}$ and slope $r_{\text {in }}$ and the output position $r_{\text {out }}$ and slope $r_{\text {out }}^{\prime}$ of a ray passing through a Homogeneous Dielectric.

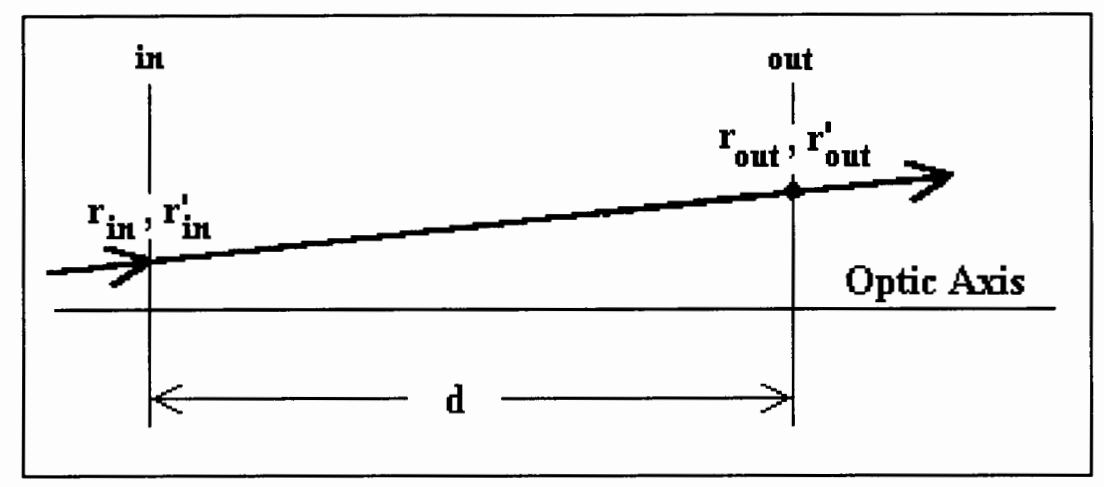

Figure 2. Ray propagation through a Homogeneous Dielectric of length d.

The output and input rays are related by:

$$
\begin{gathered}
r_{\text {out }}=1 \cdot r_{\text {in }}+d \cdot r_{\text {in }}^{\prime}=A \cdot r_{\text {in }}+B \cdot r_{\text {in }}^{\prime} \\
r_{\text {out }}^{\prime}=0 \cdot r_{\text {in }}+1 \cdot r_{\text {in }}^{\prime}=C \cdot r_{\text {in }}+D \cdot r_{\text {in }}^{\prime} \\
\text { or } \\
{\left[\begin{array}{l}
r_{\text {out }} \\
r_{\text {out }}^{\prime}
\end{array}\right]=\left[\begin{array}{ll}
1 & d \\
0 & 1
\end{array}\right] \cdot\left[\begin{array}{l}
r_{\text {in }} \\
r_{\text {in }}^{\prime}
\end{array}\right] .}
\end{gathered}
$$

The homogeneous dielectric ray-transfer matrix $\mathbf{T}_{\mathrm{HD}}$ is 


$$
\mathbf{T}_{\mathrm{HD}}=\left[\begin{array}{ll}
1 & \mathrm{~d} \\
0 & 1
\end{array}\right]=\left[\begin{array}{ll}
\mathrm{A} & \mathrm{B} \\
\mathrm{C} & \mathrm{D}
\end{array}\right] .
$$

Note that $\mathbf{T}_{\mathrm{HD}}$ is unimodular, i.e. $\mathrm{AD}-\mathrm{BC}=1$.

"Thin" Lens Ray Propagation. Figure 3 shows two cardinal rays $r_{a}$ and $r_{b}$ passing through a "thin" Lens whose thickness is negligible.

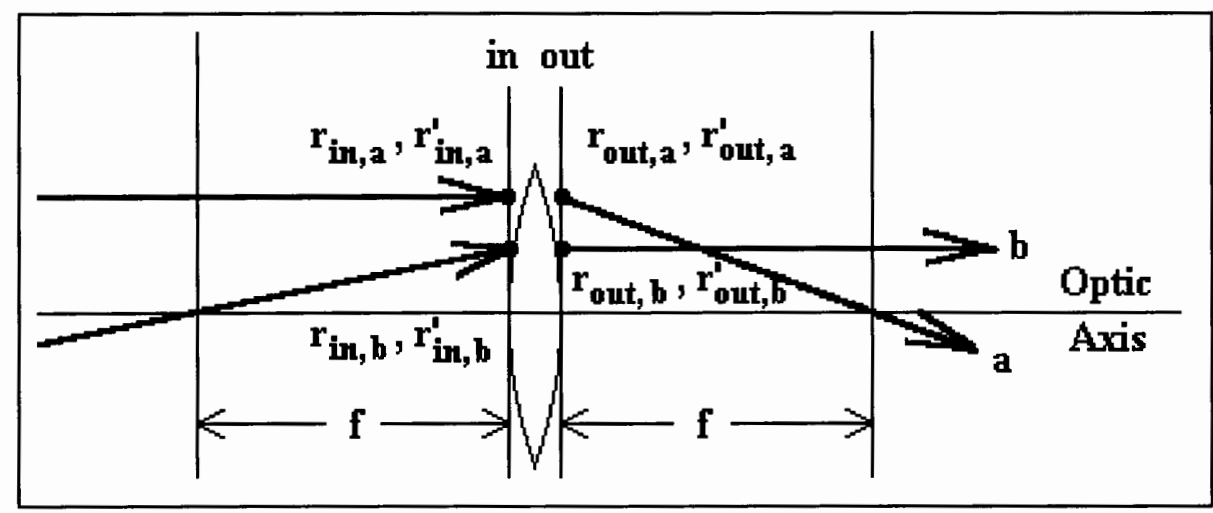

Figure 3. Ray propagation through a "Thin" Lens.

The output position equals the input position regardless of input direction, thus

$$
r_{\text {out }}=r_{\text {in }}=A \cdot r_{\text {in }}+B \cdot r_{\text {in }}^{\prime} \text {. }
$$

Therefore $\mathrm{A}=1$ and $\mathrm{B}=0$.

According to geometrical optics a ray parallel to the optic axis will pass through the back focal point $f$ as indicated by ray a in Figure 3. Here $r_{\text {in, },}^{\prime}=0$ and $\mathrm{r}_{\text {out }, \mathrm{a}}=-\mathrm{r}_{\mathrm{in}, \mathrm{a}} / \mathrm{f}$ so that

$$
r_{\text {out }, a}=-r_{i n, a} / f=C \cdot r_{i n, a}+D \cdot r_{i n, a}^{\prime}=C \cdot r_{i n, a}+D \cdot 0 \text { or } C=-1 / f .
$$

Ray $b$ passes through the front focal point $f$ and exits parallel to the optic axis. Here $r_{\text {out }, \mathrm{b}}^{\prime}=0$ so that

$$
r_{\text {out }, b}^{\prime}=0=-1 / f \cdot r_{i n, b}+D \cdot r_{\text {in, }} b \text { and } r_{\text {in, }, b}=r_{\text {in, }, b} / f .
$$

Therefore $\mathrm{D}=1$ and the ray-transfer matrix of a "Thin" Lens $\mathbf{T}_{\mathrm{TL}}$ is 


$$
\mathbf{T}_{\mathrm{TL}}=\left[\begin{array}{cc}
1 & 0 \\
-1 / \mathrm{f} & 1
\end{array}\right]=\left[\begin{array}{ll}
\mathrm{A} & \mathrm{B} \\
\mathrm{C} & \mathrm{D}
\end{array}\right]
$$

Note that $\mathbf{T}_{\mathrm{TL}}$ is unimodular.

Spherical Mirror Ray Propagation. Figure 4 shows an incident and reflected ray upon a Spherical Mirror with radius of curvature R.

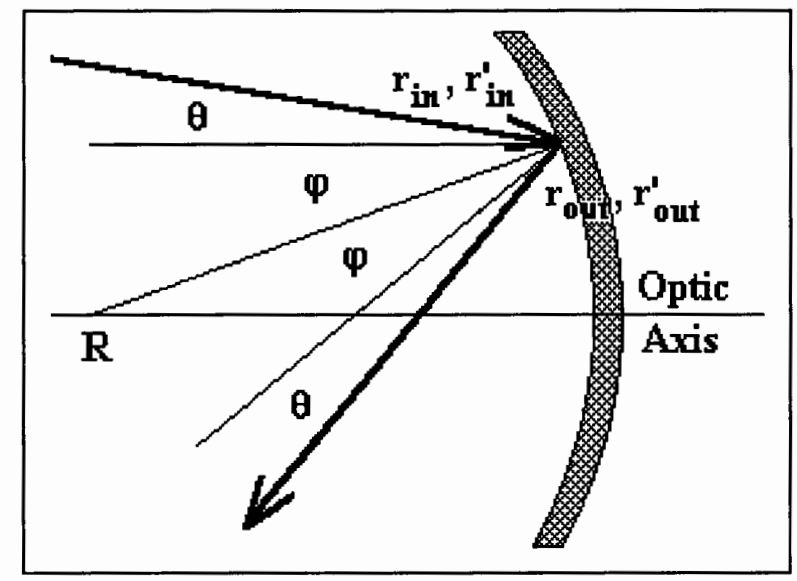

Figure 4. A Ray being reflected on a Spherical Mirror.

The input and output positions are the same so that $r_{\text {out }}=r_{\text {in }}=A \cdot r_{\text {in }}+B \cdot r_{\text {in }}^{\prime}$. Therefore $\mathrm{A}=1$ and $\mathrm{B}=0$. For the input and output slopes we have

$$
\begin{gathered}
r_{\text {in }}^{\prime}=-\tan (\theta) \approx \theta \text { and } \sin (\varphi)=r_{\text {in }} / R \approx \varphi \\
\text { and } \\
r_{\text {out }}^{\prime}=-\tan (2 \varphi+\theta) \approx-(2 \varphi+\theta), \text { or } \\
r_{\text {out }}^{\prime}=C \cdot r_{\text {in }}+D \cdot r_{\text {in }}^{\prime}=-2 / R \cdot r_{\text {in }}+1 \cdot r_{\text {in }}^{\prime} .
\end{gathered}
$$

Here $C=-2 / R$ and $D=1$ so that the spherical mirror ray-transfer matrix $\mathbf{T}_{S M}$ becomes

$$
\mathbf{T}_{\mathrm{SM}}=\left[\begin{array}{cc}
1 & 0 \\
-2 / \mathrm{R} & 1
\end{array}\right]=\left[\begin{array}{cc}
\mathrm{A} & \mathrm{B} \\
\mathrm{C} & \mathrm{D}
\end{array}\right] .
$$

Again note that $\mathbf{T}_{\mathrm{SM}}$ is unimodular. 
It is readily seen from the ray-transfer matrices $\mathbf{T}_{\mathrm{TL}}$ and $\mathbf{T}_{\mathrm{SM}}$ that reflection from a Spherical Mirror with radius of curvature R is equivalent, except the folding of the ray's path, to passage through a "Thin" Lens of focal length $f=R / 2$.

Figure 5 shows an important use of the ray-transfer matrix: that of cascading optical elements together into a single optical component of ray-transfer matrix, $\mathbf{T}_{\mathrm{SYS}}$.

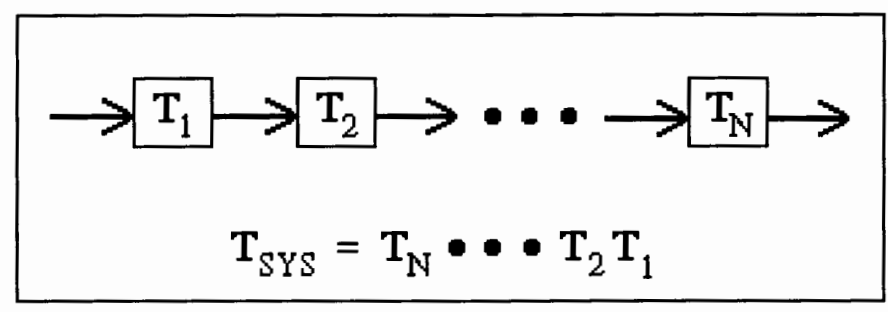

Figure 5. The cascading of Ray-Transfer Matrices.

The order of the above matrix multiplication is such that the incident ray's transfer matrix is placed to the right. This is analogous to the use of the "S" parameters in Microwave Circuit Theory.

\section{THE STABLE SPHERICAL MIRROR RESONATOR}

We will now develop the simple Spherical Mirror Resonator using the Homogeneous Dielectric and "Thin" Lens ray-transfer matrices; ultimately defining the Stability Diagram of Figure 7. The confinement condition of light rays within this resonator will be derived from two perspectives; the unbounded lens waveguide method and the self-consistent method.

The Unbounded Lens Waveguide Method 
In this method we transform the spherical mirror system into a lens waveguide and analyze the light rays' paths as they traverse the periodic sequence. Figure 6 shows an empty laser cavity with its equivalent lens waveguide comprised of an unbounded

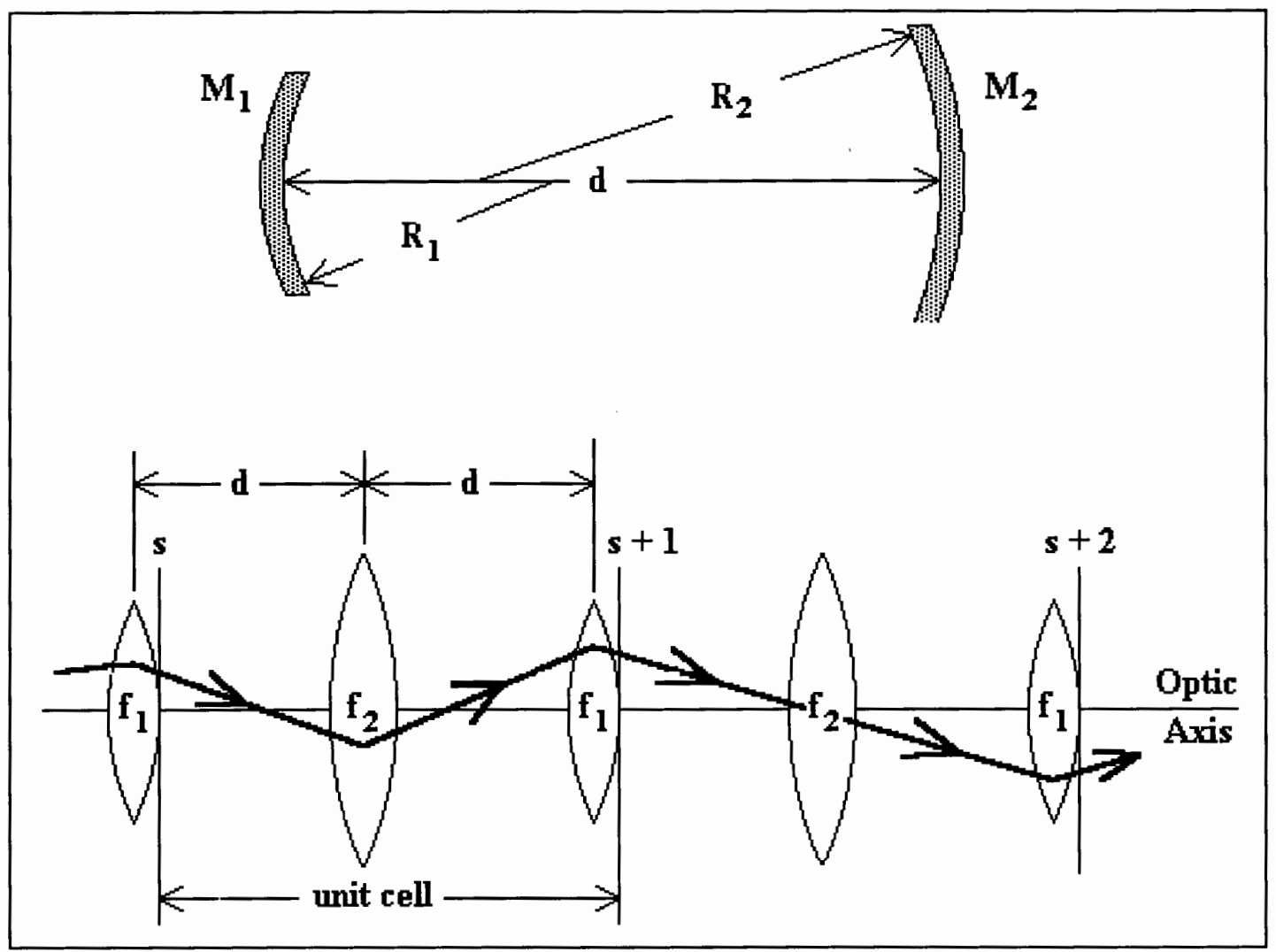

Figure 6. An empty laser cavity with its equivalent biperiodic lens sequence.

biperiodic lens sequence. We begin by making use of the ray-transfer matrices previously derived.

The ray-transfer of the unit cell is comprised of

$$
\begin{gathered}
\mathbf{T}_{\text {unit cell }}=\mathbf{T}_{\mathrm{TL}, 1} \bullet \mathbf{T}_{\mathrm{HD}} \bullet \mathbf{T}_{\mathrm{TL}, 2} \bullet \mathbf{T}_{\mathrm{HD}} \\
\text { or } \\
\mathbf{T}_{\text {unit cell }}=\left[\begin{array}{cc}
1 & 0 \\
-1 / \mathrm{f}_{1} & 1
\end{array}\right] \cdot\left[\begin{array}{ll}
1 & \mathrm{~d} \\
0 & 1
\end{array}\right] \cdot\left[\begin{array}{cc}
1 & 0 \\
-1 / \mathrm{f}_{2} & 1
\end{array}\right] \cdot\left[\begin{array}{ll}
1 & \mathrm{~d} \\
0 & 1
\end{array}\right]
\end{gathered}
$$


or finally

$$
\mathbf{T}_{\text {unit cell }}=\left[\begin{array}{cc}
1-\mathrm{d} / \mathrm{f}_{2} & \mathrm{~d}\left(2-\mathrm{d} / \mathrm{f}_{2}\right) \\
\left(\mathrm{d} / \mathrm{f}_{1}-1\right) / \mathrm{f}_{2}-1 / \mathrm{f}_{1} & \left(1-\mathrm{d} / \mathrm{f}_{1}\right)\left(1-\mathrm{d} / \mathrm{f}_{2}\right)-\mathrm{d} / \mathrm{f}_{1}
\end{array}\right] .
$$

Consider the planes denoted by $s, s+1, s+2, \cdots$ in Figure 6 . Ray propagation from one plane to the next can be written as

$$
\begin{gathered}
{\left[\begin{array}{l}
r_{s+1} \\
r_{s+1}^{\prime}
\end{array}\right]=T_{\text {unit cell }} \cdot\left[\begin{array}{c}
r_{s} \\
r_{s}^{\prime}
\end{array}\right]} \\
\text { or } \\
r_{s+1}=A \cdot r_{s}+B \cdot r_{s}^{\prime} \Rightarrow r_{s}^{\prime}=\left(r_{s+1}-A \cdot r_{s}\right) / B
\end{gathered}
$$

and

$$
r_{s+1}^{\prime}=\left(r_{s+2}-A \cdot r_{s+1}\right) / B=C \cdot r_{s}+D \cdot r_{s}^{\prime} .
$$

Substituting $r_{s}^{\prime}$ we obtain

$$
\left(r_{s+2}-A \cdot r_{s+1}\right) / B=C \cdot r_{s}+\left(r_{s+1}-A \cdot r_{s}\right) \cdot D / B
$$

Combining terms and using $\mathrm{AD}-\mathrm{BC}=1$ yields

$$
\begin{gathered}
r_{s+2}-(A+D) \cdot r_{s+1}+r_{s}=0 \\
\text { or } \\
r_{s+2}-2 b r_{s+1}+r_{s}=0, \text { where } b=(A+D) / 2=\left(1-d / f_{2}-d / f_{1}+d^{2} /\left(2 f_{1} f_{2}\right)\right) .
\end{gathered}
$$

This last equation is in equivalent form to the differential equation $r^{\prime \prime}+k r=0$

which has solutions $r(\mathrm{z})=\rho \exp \left[ \pm i(\mathrm{k})^{1 / 2} \mathrm{z}\right]$. We are thus led to try a solution in the form of $\mathrm{r}_{\mathrm{s}}=\rho e^{i \mathrm{~s} \theta}$ that when substituted into the last equation yields

$$
e^{2 i \theta}-2 \mathrm{~b} e^{i \theta}+1=0 \text {. }
$$

Thus $e^{i \theta}=\mathrm{b} \pm i\left(1-\mathrm{b}^{2}\right)^{1 / 2}$ so that $\mathrm{b}^{2} \leq 1$ and $\cos (\theta)=\mathrm{b}$. The general solution is a linear combination of the form

$$
\mathrm{r}_{\mathrm{s}}=\rho e^{i \mathrm{~s} \theta}+\rho^{*} e^{-i \mathrm{~s} \theta} \text { or } \mathrm{r}_{\mathrm{s}}=\mathrm{r}_{\max } \sin (\mathrm{s} \theta+\alpha) .
$$


The condition for ray confinement is such that $\theta$ be a real number so that the ray radius $r_{s}$ oscillates as a function of the cell number $s$ between $r_{\max }$ and $-r_{\max }$. This means that $b^{2} \leq 1$. A confined ray leads to a stable laser cavity.

There can also be the case when $b^{2}>1$. This has solutions in the form of $\mathrm{r}_{\mathrm{S}}=\mathrm{c} e^{\mathrm{s} \theta}+\mathrm{d} e^{-\mathrm{s} \theta}$, where $e^{ \pm \theta}=\mathrm{b} \pm\left(\mathrm{b}^{2}-1\right)^{1 / 2}$. Since the magnitude of either $e^{+\theta}$ or $e^{-\theta}$ exceeds unity, the ray radius will increase as a function of (distance) s. The ray is unconfined which leads to an unstable laser cavity.

In terms of system parameters, $b^{2} \leq 1$ or $|b| \leq 1$ can be written as

$$
\begin{gathered}
-1 \leq\left(1-d / f_{2}-d / f_{1}+d^{2} /\left\{2 f_{1} f_{2}\right\}\right) \leq 1 \\
\text { or } \\
0 \leq\left(1-d /\left(2 f_{1}\right)\right)\left(1-d /\left(2 f_{2}\right)\right) \leq 1 .
\end{gathered}
$$

When substituting $f_{1}=R_{1} / 2$ and $f_{2}=R_{2} / 2$ we obtain the confinement condition for simple spherical mirror resonators,

$$
0 \leq\left(1-d / R_{1}\right)\left(1-d / R_{2}\right) \leq 1 .
$$

Figure 7 shows a graphic representation of the confinement condition given in the above equation [12]. The shaded areas represent high diffraction loss where $b^{2}>1$. Here the beam is not well confined and spills over the mirror's edge. Whereas in the confined areas the beam satisfies $b^{2} \leq 1$ and a low loss condition occurs for optical resonance.

Note that when the mirror curvatures are equal $\left(R_{1}=R_{2}\right)$ the system is symmetrical and lies along the diagonal line depicted by the progression of 1 to 6 in Figure 7. These various configurations are shown in Figure 8. 


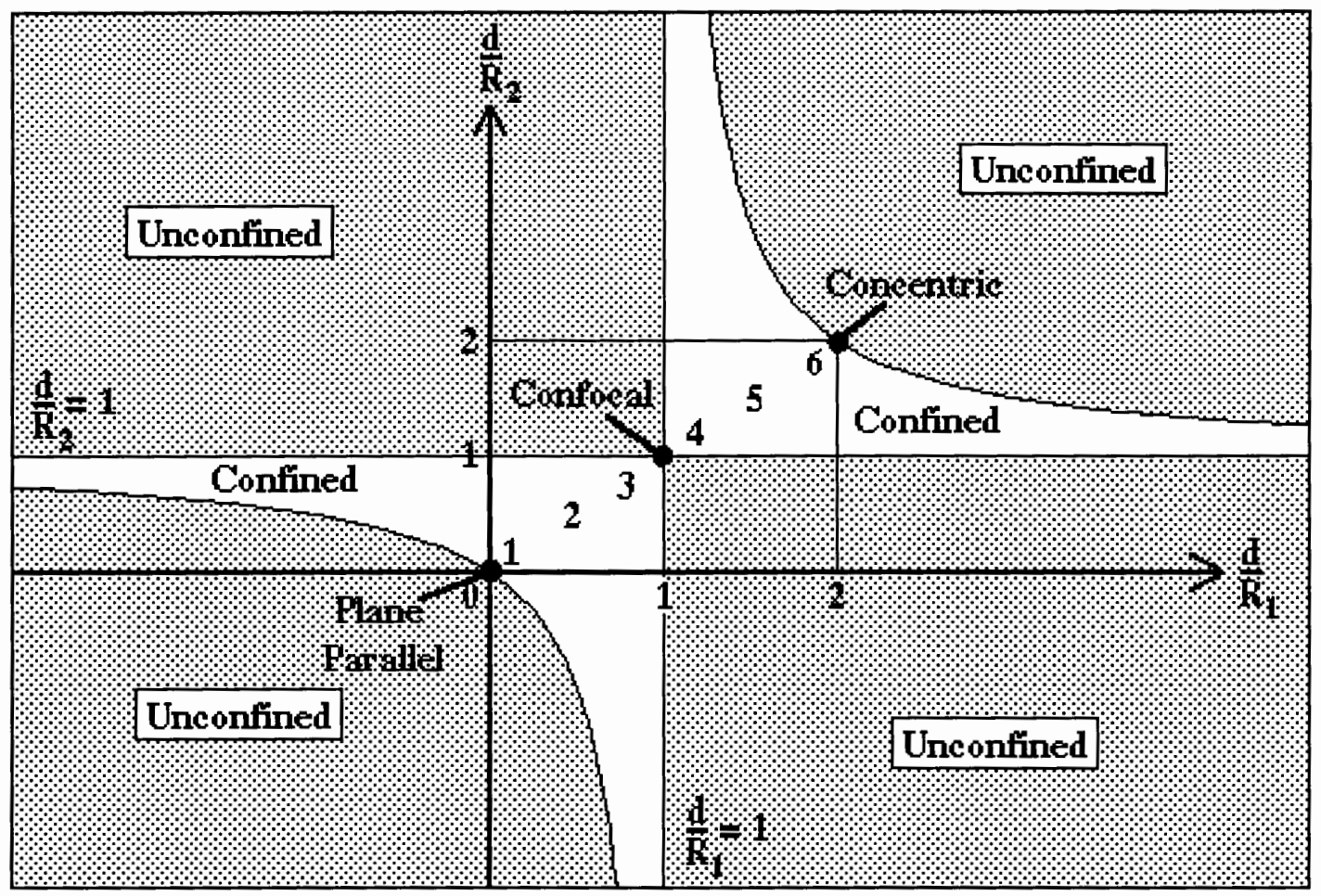

Figure 7. The Beam Confinement (or Stability) Diagram for optical resonators.
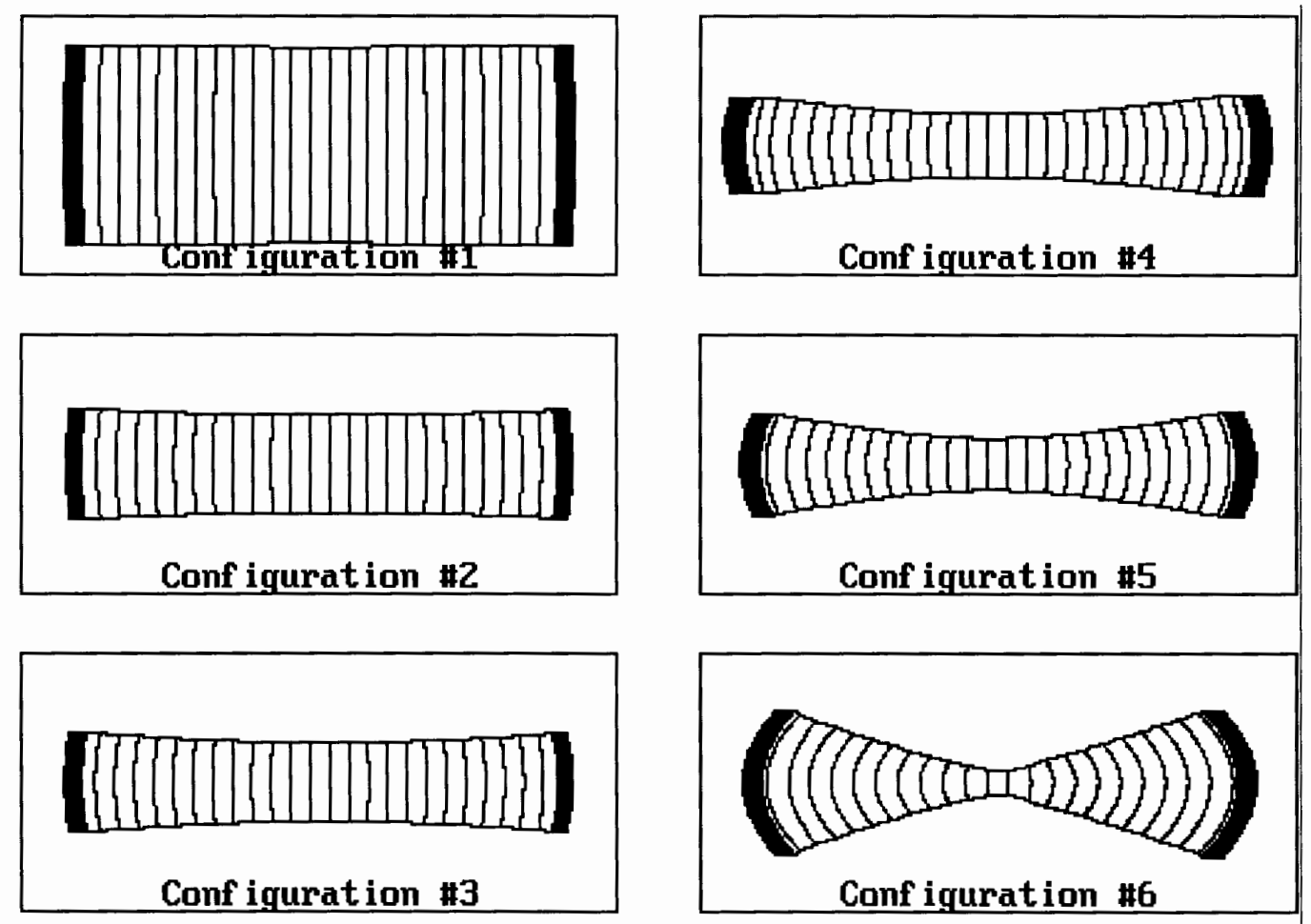

Figure 8. A progressive selection of Symmetric Mirror curvatures taken from Figure 7. 
The Gaussian Beam. The Gaussian beam wavefronts, shown in each configuration of Figure 7, all have the same curvature as that of the radius of curvature of their respective mirrors. The beam is reflected back on itself and will retrace its path back and forth within the resonator. The beam then can exist self-consistently within the cavity satisfying the Helmholtz equation $\left(\nabla^{2} \mathbf{E}+\mathrm{k}^{2}(\mathbf{r}) \mathbf{E}=0\right)$ as well as the boundary conditions imposed by the mirrors. The Gaussian beam is a mode of the spherical-mirror resonator provided that its phase also retraces itself.

The Fundamental Gaussian beam is given by

$$
\mathrm{E}(\mathrm{x}, \mathrm{y}, \mathrm{z})=\mathrm{E}_{\mathrm{o}}\left[\frac{\mathrm{w}_{\mathrm{o}}}{\mathrm{w}(\mathrm{z})}\right] \exp \left[-\frac{\mathrm{r}^{2}}{\mathrm{w}^{2}(\mathrm{z})}-i\left\{\mathrm{kz}+\frac{\mathrm{kr}^{2}}{2 \mathrm{R}(\mathrm{z})}-\eta(\mathrm{z})\right\}\right],
$$

where $\mathrm{E}(\mathrm{x}, \mathrm{y}, \mathrm{z})=$ the Electric Field,

$$
\begin{aligned}
\mathrm{E}_{\mathrm{O}}= & \text { the initial amplitude, } \\
\mathrm{w}_{\mathrm{O}}= & \text { the waist radius. The waist radius } \mathrm{w}_{\mathrm{o}} \text { is called the spot size, } \\
\mathrm{w}(\mathrm{z})= & \text { the radial distance } \mathrm{r} \text { at which the field amplitude is down by a factor of } \\
& 1 / e \text { compared to its value on the } \mathrm{z} \text { axis, } \\
\mathrm{r}= & \left(\mathrm{x}^{2}+\mathrm{y}^{2}\right)^{1 / 2} ; \text { the radial distance, } \\
i= & (-1)^{1 / 2} ; \text { the imaginary number, } \\
\eta(\mathrm{z})= & \text { the Guoy phase shift }=\text { the phase retardation relative to a plane wave, } \\
\mathrm{k}= & 2 \pi /(\mathrm{n} \lambda) ; \text { the wavenumber and } \mathrm{n}=\text { the index of refraction, and } \\
\mathrm{R}(\mathrm{z})= & \text { the radius of curvature of the wavefronts. }
\end{aligned}
$$

Some of these parameters are defined as

$$
\begin{aligned}
\mathrm{w}^{2}(\mathrm{z}) & =\mathrm{w}_{\mathrm{o}}^{2}\left[1+\left(\lambda \mathrm{z} /\left(\pi \mathrm{w}_{\mathrm{o}}^{2} \mathrm{n}\right)\right)^{2}\right]=\mathrm{w}_{\mathrm{o}}^{2}\left[1+\left(\mathrm{z} / \mathrm{z}_{\mathrm{o}}\right)^{2}\right] \\
\mathrm{R}(\mathrm{z}) & =\mathrm{z}\left[1+\left(\pi \mathrm{w}_{\mathrm{o}}^{2} \mathrm{n} /(\lambda \mathrm{z})\right)^{2}\right]=\mathrm{z}\left[1+\left(\mathrm{z}_{\mathrm{o}} / \mathrm{z}\right)^{2}\right] \\
\eta(\mathrm{z}) & =\tan ^{-1}\left[\lambda \mathrm{z} /\left(\pi \mathrm{w}_{\mathrm{o}}^{2} \mathrm{n}\right)\right]=\tan ^{-1}\left(\mathrm{z} / \mathrm{z}_{\mathrm{o}}\right) \\
\mathrm{z}_{\mathrm{o}} & \equiv \pi \mathrm{w}_{\mathrm{o}}^{2} \mathrm{n} / \lambda
\end{aligned}
$$

where $\mathrm{z}_{\mathrm{O}}=$ the confocal parameter having the following properties at the $\mathrm{z}=\mathrm{z}_{\mathrm{o}}$ plane: 
a) the intensity on the beam axis is $1 / 2$ the $z=0$ peak value;

b) the beam radius is $(2)^{1 / 2}$ larger than $w_{o}$. (the beam area is doubled vs. at $\mathrm{z}=0$ );

c) the phase on the beam axis is retarded by 90 degrees to that of a plane wave; and

d) the radius of curvature is at its smallest value, $R_{\min }=2 z_{0}$.

The confocal parameter, sometimes known as the depth of focus, is a convenient measure of the divergence of an output beam. It is also an estimate of where Fresnel diffraction ends $\left(\mathrm{z}<\mathrm{z}_{\mathrm{o}}\right)$ and where Fraunhofer diffraction begins $\left(\mathrm{z}>\mathrm{z}_{\mathrm{o}}\right)$.

\section{The Self Consistent Method}

In this method of determining the confinement condition we will make use of the complex beam radius $\mathrm{q}(\mathrm{z})$ which enables one to determine the beam radius $\mathrm{w}(\mathrm{z})$ and its radius of curvature $\mathrm{R}$ at any $\mathrm{z}$ plane. It is defined as

$$
\frac{1}{q(z)}=\frac{1}{R(z)}-\frac{i \lambda}{n \pi w^{2}(z)} .
$$

The ABCD Law. The usefulness of the q parameter is found when applying the $\mathrm{ABCD}$ law where

$$
\mathrm{q}_{\text {out }}=\frac{A q_{\text {in }}+\mathrm{B}}{C q_{\text {in }}+D}=\text { The ABCD Law. }
$$

$\mathrm{A}, \mathrm{B}, \mathrm{C}$ and $\mathrm{D}$ are the elements of the transfer matrix $\mathbf{T}$ and the output and input Gaussian beams are characterized by $\mathrm{q}_{\text {out }}$ and $\mathrm{q}_{\text {in }}$ respectively. Full characterization requires additional knowledge of the beam axis and intensity.

Gaussian beam propagation through a complex arbitrary paraxial optical system can be determined if one knows either $q_{\text {in }}$ or $q_{\text {out }}$ and the system's transfer matrix $\mathbf{T}_{\mathrm{SYS}}$. The beam radius of curvature $R(z)$ and waist $w(z)$ at any $\mathrm{z}$ plane can then be recovered according to the above two equations. 
We will now apply the $\mathrm{ABCD}$ law to a generalized resonator by what is called the self consistent method. A stable resonant eigenmode is one which reproduces itself after one round trip. An arbitrary reference plane is selected and the $A B C D$ elements for one complete round trip are then used in the $\mathrm{ABCD}$ law. At the reference plane the complex beam parameter $\mathrm{q}=\mathrm{q}_{\text {in }}=\mathrm{q}_{\text {out }}$ if the beam is to reproduce itself. We require that $q=(A q+B) /(C q+D)$. Solving for $1 / q$ using $A D-B C=1$ yields

$$
1 / \mathrm{q}=\left[(\mathrm{D}-\mathrm{A}) \pm i\left(4-(\mathrm{D}+\mathrm{A})^{2}\right)^{1 / 2}\right] / 2 \mathrm{~B} \text {. }
$$

Since 1/q must be complex, due to the waist being finite size, we have $4-(\mathrm{D}+\mathrm{A})^{2}>0$ or $\mid(\mathrm{A}+\mathrm{D}) / 2 \mathrm{I} \leq 1$. This is the confinement condition earlier denoted as $|b| \leq 1$. The radius of curvature $R$ and the waist $w$ at the reference plane are

$$
\begin{gathered}
\mathrm{R}=2 \mathrm{~B} /(\mathrm{D}-\mathrm{A}) \text { and } \\
\mathrm{w}=(\lambda / \pi n)^{1 / 2}|\mathrm{~B}|^{1 / 2} /\left[1-((\mathrm{D}+\mathrm{A}) / 2)^{2}\right]^{1 / 4} .
\end{gathered}
$$

\section{The Paraxial Wave Equation}

The paraxial wave equation is an approximation to the scalar wave equation which is derived from Maxwell's equations in free space. We begin with the scalar wave equation in the form

$$
\left[\nabla^{2}+k^{2}\right] E(x, y, z)=0,
$$

where $\mathrm{E}(\mathrm{x}, \mathrm{y}, \mathrm{z})$ is the phasor amplitude of a field distribution that is sinusoidal in time. The flow of energy is predominantly along a single direction, the $\mathrm{z}$ axis. The primary spatial dependence of $\mathrm{E}(\mathrm{x}, \mathrm{y}, \mathrm{z})$ will be an $\exp (-i \mathrm{kz})$ variation which has a spatial period of one wavelength $\lambda$ in the $\mathrm{z}$ direction. The transverse variations due to diffraction and propagation are usually slow compared one optical cycle as in the plane-wave exp(-ikz) variation. To get better resolution of the transverse dependence we write $E(x, y, z)$ in the form

$$
\mathrm{E}(\mathrm{x}, \mathrm{y}, \mathrm{z})=\psi(\mathrm{x}, \mathrm{y}, \mathrm{z}) e^{-i \mathrm{kz}},
$$


where $\psi(\mathrm{x}, \mathrm{y}, \mathrm{z})=$ a complex scalar wave amplitude which describes the transverse profile of the beam. Substituting this into the scalar wave equation yields, in Cartesian coordinates, the reduced equation

$$
\frac{\partial^{2} \psi}{\partial^{2} x}+\frac{\partial^{2} \psi}{\partial^{2} y}+\frac{\partial^{2} \psi}{\partial^{2} z}-2 i k \frac{\partial \psi}{\partial z}=0
$$

The $\mathrm{z}$ dependence in the transverse direction is assumed slow enough that

$$
\left|\frac{\partial^{2} \psi}{\partial^{2} \mathrm{z}}\right| \ll\left|2 \mathrm{k} \frac{\partial \psi}{\partial \mathrm{z}}\right| .
$$

This is the slowly varying envelop approximation or paraxial approximation. By dropping the second partial derivative in $\mathrm{z}$, the exact scalar wave equation becomes the paraxial wave equation

$$
\frac{\partial^{2} \psi}{\partial^{2} x}+\frac{\partial^{2} \psi}{\partial^{2} y}-2 i k \frac{\partial \psi}{\partial z}=0 .
$$

More generally this equation becomes

$$
\nabla^{2} \psi(\mathrm{s}, \mathrm{z})-2 i \mathrm{k} \frac{\partial \psi(\mathrm{s}, \mathrm{z})}{\partial \mathrm{z}}=0,
$$

where $s$ denotes either the $\mathrm{x}, \mathrm{y}$ or $\mathrm{r}, \theta$ coordinates in rectangular or cylindrical coordinates respectively and $\nabla_{t}^{2}$ is the laplacian operator operating on these coordinates in the transverse plane.

\section{OPTICAL RESONATOR ALGEBRA}

We now will determine the Gaussian beam whose curvature matches the mirror curvatures $R_{1}$ and $R_{2}$ at the location of the mirrors $M_{1}$ and $M_{2}$ respectively. We will find the confocal parameter $z_{0}$ and the waist $w_{0}$ from the system's parameters $\left(d, R_{1}\right.$ and $\left.R_{2}\right)$. Once $z_{0}$ and $w_{0}$ are known the Gaussian beam is thus defined except for the initial amplitude $\mathrm{E}_{0}$. The beam direction is taken along the $\mathrm{z}$ axis. The location of $\mathrm{w}_{\mathrm{O}}$ is where $\mathrm{z}=0$. The locations of $\mathrm{M}_{1}$ and $\mathrm{M}_{2}$ are where $\mathrm{z}=\mathrm{z}_{1}$ and $\mathrm{z}=\mathrm{z}_{2}$ respectively. 
To determine the waist radii at the mirrors of given $R_{1}$ and $R_{2}$ we first find $z_{0}$. Then the waist $\mathrm{w}_{0}$ is found. Finally we calculate the waist radii $\mathrm{w}_{1}$ and $\mathrm{w}_{2}$. We begin with the equations

$$
\begin{aligned}
& \mathrm{R}_{\mathrm{i}}=\mathrm{z}_{\mathrm{i}}\left[1+\left(\mathrm{z}_{0} / \mathrm{z}_{\mathrm{i}}\right)^{2}\right] \Rightarrow \mathrm{z}_{\mathrm{i}}=\mathrm{R}_{\mathrm{i}} / 2 \pm\left(\mathrm{R}_{\mathrm{i}}^{2}-4 \mathrm{z}_{0}^{2}\right) / 2 ; \mathrm{i}=1,2 \\
& \text { and } \\
& \mathrm{d}=\mathrm{z}_{2}-\mathrm{z}_{1} .
\end{aligned}
$$

Through-out this section on resonator algebra, the mirror curvature $R_{1}$ or $R_{2}$ is positive if the center of curvature is to the left of the mirror and negative otherwise. Solving for $\mathrm{z}_{0}$ we have

$$
z_{0}=\left[-d\left(R_{1}+d\right)\left(R_{2}-d\right)\left(R_{2}-R_{1}-d\right) /\left(R_{2}-R_{1}-2 d\right)^{2}\right]^{1 / 2} .
$$

The waist is given by

$$
\mathrm{w}_{0}=\left(\lambda \mathrm{z}_{0} / \pi \mathrm{n}\right)^{1 / 2} .
$$

The waist radii at the mirrors $\mathrm{M}_{1}$ and $\mathrm{M}_{2}$ are given by

$$
\mathrm{w}_{\mathrm{i}}=\mathrm{w}_{0}\left[1+\left(\mathrm{z}_{\mathrm{i}} / \mathrm{z}_{0}\right)^{2}\right]^{1 / 2} ; ; \mathrm{i}=1,2 .
$$

The following discussion will involve the Symmetrical Mirror Resonator $\left(\mathrm{R}_{1}=\mathrm{R}_{2}\right)$. Three special cases will be investigated: the Confocal Resonator; the Concentric Resonator; and the Plane-Parallel Resonator.

\section{The Symmetric Mirror Resonator}

This family of resonators lie along the diagonal line depicted in the Stability

Diagram of Figure 7 by the linear progression from 1 to 6 . This is where both mirrors are identical both being concave $\left(R=R_{1}=R_{2}>0\right)$.

We must remember to redefine the radius of mirror curvature $R_{1}$ or $R_{2}$ as positive if the center of curvature is to the left of the mirror and negative otherwise. Therefore we put $R=-R_{1}=R_{2}$ in the above equation for $z_{0}$ to yield

$$
\mathrm{z}_{0}=[\mathrm{d}(2 \mathrm{R}-\mathrm{d})]^{1 / 2 / 2} \text {. }
$$


The waist is given by

$$
\mathrm{w}_{0}=\left(\lambda \mathrm{z}_{0} / \pi \mathrm{n}\right)^{1 / 2}=(\lambda / \pi \mathrm{n})^{1 / 2}\left[\left(\mathrm{Rd}-\mathrm{d}^{2} / 2\right) / 2\right]^{1 / 4} .
$$

The waist radii at the mirrors are given by

$$
\mathrm{w}_{\mathrm{i}}=\mathrm{w}_{0}\left[1+\left(\mathrm{z}_{\mathrm{i}} / \mathrm{z}_{0}\right)^{2}\right]^{1 / 2} .=(\lambda \mathrm{d} / 2 \pi \mathrm{n})^{1 / 2}\left[2 \mathrm{R}^{2}(\mathrm{~d}(\mathrm{R}-\mathrm{d} / 2))\right]^{1 / 4} ; \mathrm{i}=1,2
$$

where $z_{1}=-d / 2$ and $z_{2}=d / 2$.

The Confocal Resonator. This is a special symmetrical resonator where the radii of curvature of both concave mirrors equals the cavity length d. Figure 9 depicts this type of resonator.

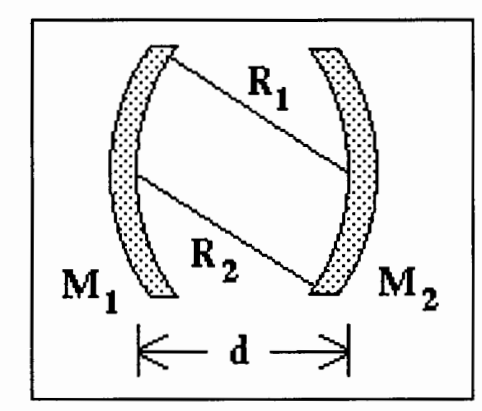

Figure 9. The Confocal Resonator where $\mathrm{R}_{1}=\mathrm{R}_{2}=\mathrm{d}$.

Here we put $\mathrm{R}=\mathrm{d}$ into the above equation for $\mathrm{z}_{0}$ and obtain

$$
\mathrm{z}_{0}=[\mathrm{d}(2 \mathrm{R}-\mathrm{d})]^{1 / 2 / 2} \Rightarrow\left(\mathrm{z}_{0}\right)_{\text {conf }}=\mathrm{d} / 2 .
$$

The waist at $\mathrm{z}=0$ becomes

$$
\left(\mathrm{w}_{0}\right)_{\mathrm{conf}}=\left(\lambda\left(\mathrm{z}_{0}\right)_{\text {conf }} / \pi \mathrm{n}\right)^{1 / 2}=(\lambda \mathrm{d} / 2 \pi \mathrm{n})^{1 / 2} .
$$

The waist at the mirrors becomes

$$
\left(\mathrm{w}_{\mathrm{i}}\right)_{\text {conf }}=\left(\mathrm{w}_{0}\right)_{\text {conf }}\left[1+\left(\mathrm{z}_{\mathrm{i}} /\left(\mathrm{z}_{0}\right)_{\text {conf }}\right)^{2}\right]^{1 / 2}=(2)^{1 / 2}\left(\mathrm{w}_{0}\right)_{\text {conf }}=(\lambda \mathrm{d} / \pi \mathrm{n})^{1 / 2} ; \mathrm{i}=1,2
$$

where $\mathrm{z}_{1}=-\mathrm{z}_{0}$ and $\mathrm{z}_{2}=\mathrm{z}_{0}$.

In the Confocal Resonator the waist radii $\left(\mathrm{w}_{1,2}\right)_{\text {conf }}$ is at the minimum value.

The Concentric Resonator. This is another special symmetrical resonator where the center of curvature of each concave mirror coincides. Figure 10 illustrates this resonator. 
We put $\mathrm{R}=\mathrm{d} / 2$ into the equation for $\mathrm{z}_{0}$ and obtain

$$
\mathrm{z}_{0}=[\mathrm{d}(2 \mathrm{R}-\mathrm{d})]^{1 / 2 / 2} \Rightarrow\left(\mathrm{z}_{0}\right)_{\text {conc }}=0 .
$$

The waist at $\mathrm{z}=0$ becomes

$$
\left(\mathrm{w}_{0}\right)_{\mathrm{conc}}=\left(\lambda\left(\mathrm{z}_{0}\right)_{\mathrm{conc}} / \pi \mathrm{n}\right)^{1 / 2}=0 .
$$

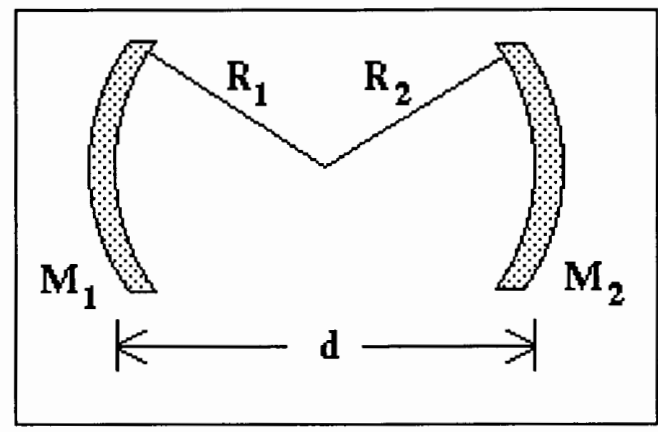

Figure 10. The Concentric Resonator where $\mathrm{R}_{1}=\mathrm{R}_{2}=\mathrm{d} / 2$.

The waist at the mirrors becomes

$$
\left(\mathrm{w}_{\mathrm{i}}\right)_{\text {conc }}=\left(\mathrm{w}_{0}\right)_{\text {conc }}\left[1+\left(\mathrm{z}_{\mathrm{i}} /\left(\mathrm{z}_{0}\right)_{\text {conc }}\right)^{2}\right]^{1 / 2}=\infty ; \mathrm{i}=1,2
$$

where $\mathrm{z}_{1}=-\mathrm{d} / 2$ and $\mathrm{z}_{2}=\mathrm{d} / 2$.

In the Concentric Resonator the waist radii $\left(\mathrm{w}_{1,2}\right)_{\mathrm{conc}}$ is at the maximum value and with $\left(\mathrm{w}_{0}\right)_{\text {conc }}=0$ implying a maximum beam divergence. This is analogous to a spherical wave. The Concentric Resonator is on the border line of the confined and unconfined regions of the Confinement Diagram.

The Plane-Parallel Resonator. This is another special symmetrical resonator where the radii of curvature of both plane mirrors equal infinity. Figure 11 illustrates this type of resonator.

We put $\mathrm{R}=\infty$ into the equation for $\mathrm{z}_{0}$ and obtain

$$
\mathrm{z}_{0}=[\mathrm{d}(2 \mathrm{R}-\mathrm{d})]^{1 / 2 / 2} \Rightarrow\left(\mathrm{z}_{0}\right)_{\text {plane }}=\infty .
$$

The waist at $\mathrm{z}=0$ becomes

$$
\left(\mathrm{w}_{0}\right)_{\text {plane }}=\left(\lambda\left(\mathrm{z}_{0}\right)_{\text {plane }} / \pi \mathrm{n}\right)^{1 / 2}=\infty .
$$




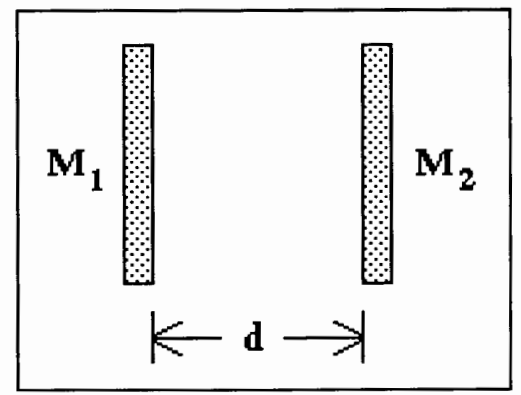

Figure 11. The Plane-Parallel Resonator where $\mathrm{R}_{1}=\mathrm{R}_{2}=\infty$.

The waist at the mirrors becomes

$$
\left(\mathrm{w}_{\mathrm{i}}\right)_{\text {plane }}=\left(\mathrm{w}_{0}\right)_{\text {plane }}\left[1+\left(\mathrm{z}_{\mathrm{i}} /\left(\mathrm{z}_{0}\right)_{\text {plane }}\right)^{2}\right]^{1 / 2}=\infty ; \mathrm{i}=1,2
$$

where $\mathrm{z}_{1}<0$ and $\mathrm{z}_{2}>0$

In the Plane-Parallel Resonator the waist radii $\left(\mathrm{w}_{1,2}\right)_{\text {plane }}$ is also at the maximum value and with the waist $\left(\mathrm{w}_{0}\right)_{\text {plane }}=\infty$ implying a minimum beam divergence. This is analogous to a plane wave. The Plane-Parallel Resonator is on the border of the confined and unconfined regions of the Confinement Diagram.

It is helpful to plot $\left(\pi \mathrm{w}^{2} / \lambda \mathrm{d}\right)^{1 / 2} \mathrm{vs}$. $d / \mathrm{R}$ to get a feel for the way the waist at the mirrors vary with $d / R$ by keeping the mirror curvatures constant while changing the mirror spacing. A plot of this function is shown in Figure 12.

The Half-Symmetric Resonator. In this resonator one of the mirrors is plane $(R=\infty)$ and the other is concave $\left(R=R_{2}\right)$. The allowed value for $d / R_{2}$ must be between 0 and 1 as can be seen in the Confinement Diagram of Figure 6. This resonator can be transformed into a symmetric resonator by substituting a mirror identical to the concave one for the plane mirror. The cavity length must then be doubled, i.e. $d \Rightarrow 2 d$.

The beam waist is located on the plane mirror, say mirror $\mathrm{M}_{1}$. In this case the expression for the waist radius at the plane and concave mirrors is given by

$$
\begin{gathered}
\mathrm{w}_{0}=\mathrm{w}_{1}=(\lambda \mathrm{d} / \pi \mathrm{n})^{1 / 2}\left[\left(1-\mathrm{d} / \mathrm{R}_{2}\right) /\left(\mathrm{d} / \mathrm{R}_{2}\right)\right]^{1 / 4} \\
\text { and } \\
\mathrm{w}_{2}=(\lambda \mathrm{d} / \pi \mathrm{n})^{1 / 2}\left[1 /\left(\left(1-\mathrm{d} / \mathrm{R}_{2}\right)\left(\mathrm{d} / \mathrm{R}_{2}\right)\right)\right]^{1 / 4}
\end{gathered}
$$




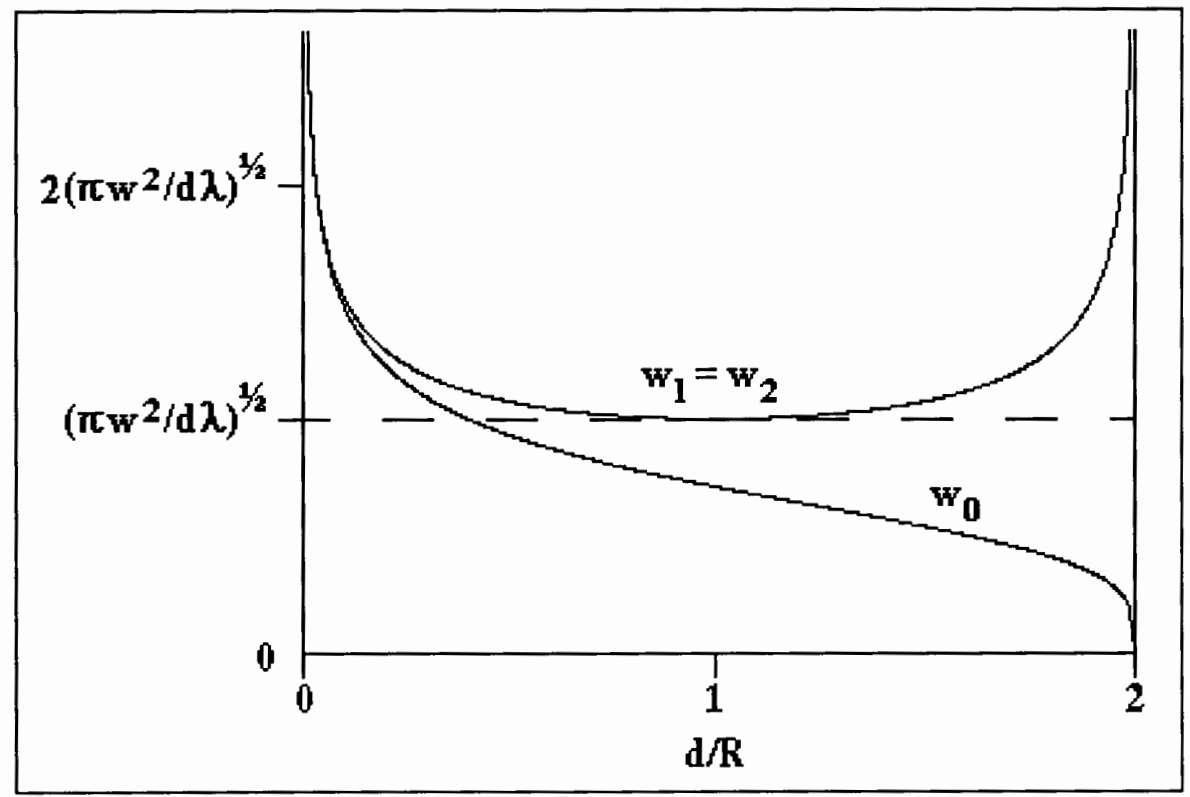

Figure 12. The $(\pi / \lambda d)^{1 / 2}$ scaled beam radius at the waist, $\mathrm{w}_{0}$ and at the mirrors, $w_{1}=w_{2}$, for a stable Symmetric Resonator as a function of $d / R$.

Again we plot $\left(\pi w^{2} / \lambda d\right)^{1 / 2} v s . d / R_{2}$ and see how the beam waist varies as one keeps the mirror curvature constant while changing the mirror distance. This is shown in Figure 13.

\section{HIGHER-ORDER TRANSVERSE MODES}

Modes of a resonator are different intensity distributions that retrace themselves when reflected between the resonator mirrors. Each mode's wave front matches the curvature of the mirrors at the mirrors.

We will discuss the Hermite-Gaussian beam which is used in rectangular geometry and the Laguerre-Gaussian beam which is used in cylindrical geometry.

\section{Hermite-Gaussian Modes}

These modes are the most widely used for the complete solution set to the paraxial wave equation since most lasers exhibit $\mathrm{x}, \mathrm{y}$ astigmatism. Siegman shows that by solving 


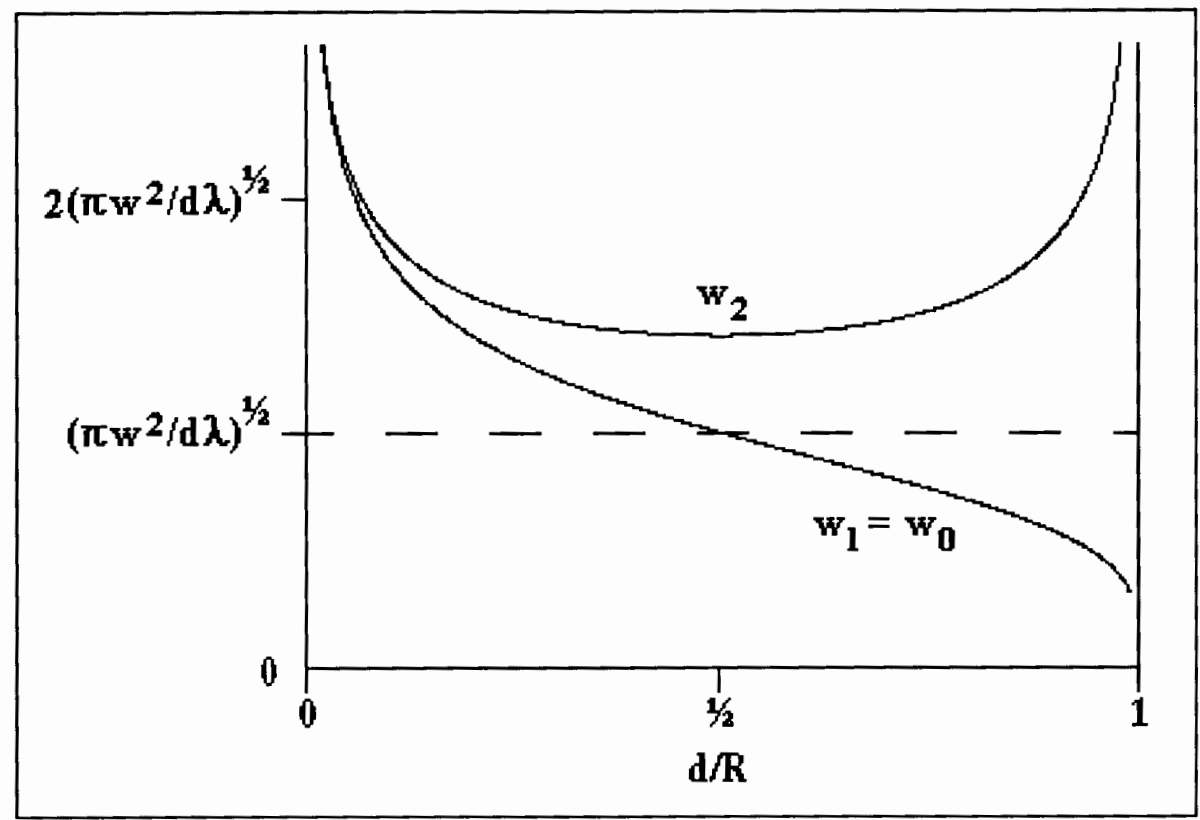

Figure 13. The $(\pi / \lambda d)^{1 / 2}$ scaled beam radius at the waist, $w_{0}=w_{1}$ at the Plane Mirror and at the Concave Mirror, $w_{2}$, for a stable Half Symmetric Resonator as a function of $d / R$.

the paraxial wave equation in Cartesian coordinates one obtains the Hermit-Gaussian modes [13]. The result is

$$
\begin{aligned}
& \mathrm{E}(\mathrm{x}, \mathrm{y}, \mathrm{z})_{\mathrm{n}, \mathrm{m}}=\left(\mathrm{E}_{\mathrm{o}}\right)_{\mathrm{n}, \mathrm{m}}\left[\frac{\mathrm{w}_{\mathrm{O}}}{\mathrm{w}(\mathrm{z})}\right] \mathrm{H}_{\mathrm{n}}\left[\frac{(2)^{1 / 2} \mathrm{x}}{\mathrm{w}(\mathrm{z})}\right] \mathrm{H}_{\mathrm{m}}\left[\frac{(2)^{1 / 2} \mathrm{y}}{\mathrm{w}(\mathrm{z})}\right] \\
& \times \exp \left[-\frac{\mathrm{x}^{2}+\mathrm{y}^{2}}{\mathrm{w}^{2}(\mathrm{z})}-i\left\{\mathrm{kz}+\mathrm{k}\left[\frac{\mathrm{x}^{2}+\mathrm{y}^{2}}{2 \mathrm{R}(\mathrm{z})}\right]-(\mathrm{n}+\mathrm{m}+1) \eta(\mathrm{z})\right],\right.
\end{aligned}
$$

where the integers $n, m \geq 0$ are the $x, y$ modal indices respectively and the functions $H_{n}$ and $\mathrm{H}_{\mathrm{m}}$ are the Hermite polynomials of order $\mathrm{n}$, m respectively. All other variables are as previously defined. These modes exhibit $\mathrm{x}, \mathrm{y}$ symmetry about the $\mathrm{x}, \mathrm{y}$ modal axes respectively.

Resonance Frequencies of the Hermite-Gaussian Modes. The phase of the (n,m) mode on the beam axis is the imaginary component of the E-Field. From the above equation we have

$$
\varphi(x=0, y=0, z)=k z-(n+m+1) \eta(z) .
$$


The phase delay of a beam in a complete round trip in a resonator of length $\mathrm{d}$ must be set to a muitiple of $2 \pi$ in order that the beam retrace itself [14]. Thus

$$
2 k d-2(n+m+1) \Delta \eta=2 \pi q,
$$

where $\mathrm{k}=$ the wave number,

$\mathrm{d}=$ the cavity length,

$\mathrm{n}, \mathrm{m}=$ the $\mathrm{x}, \mathrm{y}$ modal indices,

$\Delta \eta=\eta\left(z_{2}\right)-\eta\left(z_{1}\right)$,

$\mathrm{z}_{2}, \mathrm{z}_{1}=\mathrm{M}_{2}, \mathrm{M}_{1}$ mirror positions,

$\eta(\mathrm{z})=\tan ^{-1}\left(\mathrm{z} / \mathrm{z}_{0}\right)$,

$\mathrm{z}_{0}=$ the confocal parameter and,

$\mathrm{q}=$ the axial mode index $=0, \pm 1, \pm 2, \cdots$.

This leads to resonance frequencies of the Hermite-Gaussian modes defined by

$$
v_{n, m, q}=c /(2 d)[q+(n+m+1) \Delta \eta / \pi] .
$$

Modes of different $q$, but the same $(n, m)$, have identical intensity distributions. They are called longitudinal or axial modes. The $(\mathrm{n}, \mathrm{m})$ modes refer to the transverse $\mathrm{x}, \mathrm{y}$ dimensions and are called transverse modes.

These resonance frequencies satisfy the following properties:

a) Longitudinal modes corresponding to the transverse mode $(n, m)$ have a resonance frequency spacing of $c /(2 d)=v_{n, m, q+1}-v_{n, m, q}$;

b) All transverse modes, for which the sum of the indices $n+m$ is the same, have the same resonance frequencies; and

c) Two transverse modes $(n, m)$ and $\left(n^{\prime}, m^{\prime}\right)$ corresponding to the same longitudinal mode $\mathrm{q}$ have resonance frequencies spaced by

$$
v_{n, m, q}-v_{n^{\prime}, m^{\prime}, q}=c /(2 d)\left[(n+m)-\left(n^{\prime}+m^{\prime}\right)\right] \Delta \eta / \pi .
$$

This expression determines the frequency shift between the sets of longitudinal modes of indices $(n, m)$ and $\left(n^{\prime}, m^{\prime}\right)$. 


\section{Laguerre-Gaussian Modes}

Siegman also gives an equally valid set of complete solutions to the paraxial wave equation in cylindrical coordinates [15]. These Laguerre-Gaussian solutions have the form

$$
\begin{gathered}
E(r, \theta, z)_{p, m}=\left(E_{o}\right)_{p, m}\left[\frac{w_{o}}{w(z)}\right]\left[\frac{(2)^{1 / 2} r}{w(z)}\right]^{m} L_{p} m\left[\frac{2 r^{2}}{w^{2}(z)}\right] \\
\times \exp \left[-\left[\frac{\mathrm{r}^{2}}{w^{2}(z)}\right]-i\left\{k z+k\left[\frac{r^{2}}{2 R(z)}\right]-(2 p+m+1) \eta(z)+m \theta\right\}\right],
\end{gathered}
$$

where the integer $p \geq 0$ is the radial index and the integer $m=0, \pm 1, \pm 2, \cdots$ is the azimuthal mode index; the $\mathrm{L}_{\mathrm{p}}{ }^{\mathrm{m}}$ functions are the Laguerre polynomials; and all other quantities are as previously defined.

These modes exhibit cylindrical symmetry, with modes having circles of constant intensity in the radial direction and an $e^{i m \theta}$ variation in the azimuthal direction. Alternately, linear combinations of the $\pm m$ terms can be formed to give $\cos (m \theta)$ and/or $\sin (m \theta)$ variations, leading to $2 m$ nodal lines running radial outward from the mode axis.

Resonance Frequencies of the Laguerre-Gaussian Modes. Again, we proceed as in the Hermite-Gaussian case. The phase of the $(p, m)$ mode on the beam axis is the imaginary component of the E-Field. Thus

$$
\varphi(r=0, \theta, z)=k z-(2 p+m+1) \eta(z)-m \theta .
$$

The phase delay of a beam in a complete round trip in a resonator of length $\mathrm{d}$ must be set to a multiple of $2 \pi$ in order that the beam retrace itself. Thus

$$
2 k d-2(2 p+m+1) \Delta \eta-2 m \theta=2 \pi q,
$$

This leads to resonance frequencies of the Laguerre-Gaussian modes defined by

$$
v_{p, m, q}=c /(2 d)[q+(2 p+m+1) \Delta \eta / \pi+m \theta / \pi] .
$$

Modes of different $\mathrm{q}$, but the same $(\mathrm{p}, \mathrm{m})$, have identical intensity distributions. They are called longitudinal or axial modes. The $(\mathrm{p}, \mathrm{m})$ modes refer to the transverse $\mathrm{r}, \boldsymbol{\theta}$ dimensions and are called transverse modes. 
These resonance frequencies satisfy the following properties:

a) Longitudinal modes corresponding to the transverse mode $(\mathrm{p}, \mathrm{m})$ have a resonance frequency spacing of $c /(2 d)=v_{p, m, q+1}-v_{p, m, q}$;

b) All transverse modes, for which the sum of the indices $p+m$ is the same, have the same resonance frequencies; and

c) Two transverse modes $(\mathrm{p}, \mathrm{m})$ and $\left(\mathrm{p}^{\prime}, \mathrm{m}^{\prime}\right)$ corresponding to the same longitudinal mode q have resonance frequencies spaced by $v_{p, m, q}-v_{p^{\prime}, m^{\prime}, q}=c /(2 d)\left[\left[(2 p+m)-\left(2 p^{\prime}+m^{\prime}\right)\right] \Delta \eta / \pi+\left(m-m^{\prime}\right) \theta / \pi\right]$.

This expression determines the frequency shift between the sets of longitudinal modes of indices (p,m) and (p', m').

Since either solution set (Hermite-Gaussian or Laguerre-Gaussian) can be used, we must be able to expand the Hermite solutions in terms of Laguerre functions and vice versa.

Most laser systems incorporate rectangular geometry, such as Brewster's mirrors or tilted components, such that the beam elects to oscillate in near-Hermite-Gaussian modes. The work in this thesis solely has cylindrical symmetry with the azimuthal index $m=0$. Therefore the beam elects to oscillate in Laguerre-Gaussian $L_{p}$ modes only. 


\section{CHAPTER III}

\section{RESONATOR MIRRORS}

In this chapter we will discuss the design of the following three types of resonator mirrors: 1) the Spherical Mirror, a well known type used in most laser systems; 2) the Fresnel Mirror, not used in laser systems probably due to it's manufacturability; and 3) the Tiered Fresnel Mirror, a novel type that can be efficiently manufactured using segments of the Integrated Circuits Process. These mirrors are shown in Figure 14.

Also discussed is diffraction losses in an optical resonator system. This is important because it is a figure of merit to compare the novel Tiered Fresnel Mirror to the Spherical Mirror.

\section{THE SPHERICAL MIRROR}

The design of the Spherical Mirror is simple. It has a constant radius of curvature $\mathrm{R}$, where $\mathrm{R}$ equals the distance that is normal to the mirror's surface at the center of the mirror to it's center of curvature. This is shown in Figure 15. Also shown is the mirror's radial dimension $r$, where $r$ equals the distance that is tangent to the mirror's surface at it's center to the edge.

Different mirrors, $\mathrm{r}$ and/or $\mathrm{R}$ being different, will naturally yield a different performance in a laser system. The mirror performance or diffraction loss associated with a resonator mirror is a function proportional to the resonator Fresnel number N, where

$$
\mathrm{N} \equiv \mathrm{r}^{2} /(\lambda \mathrm{d}) .
$$



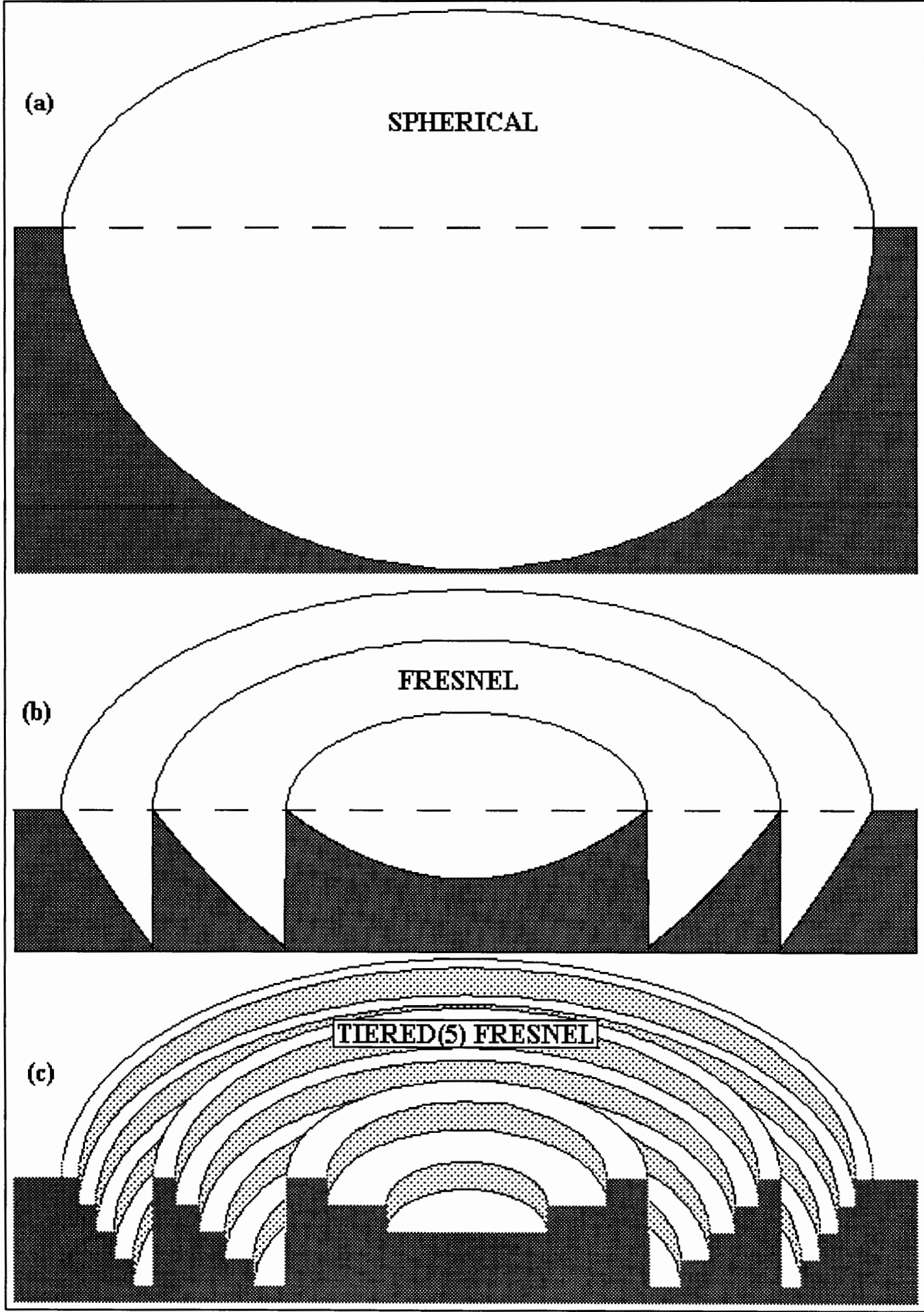

Figure 14. A 3d cross-sectional view of: a) the Spherical Mirror, b) the Fresnel Mirror, and c) the Tiered(5) Fresnel Mirror having 5 tiers per zone. 


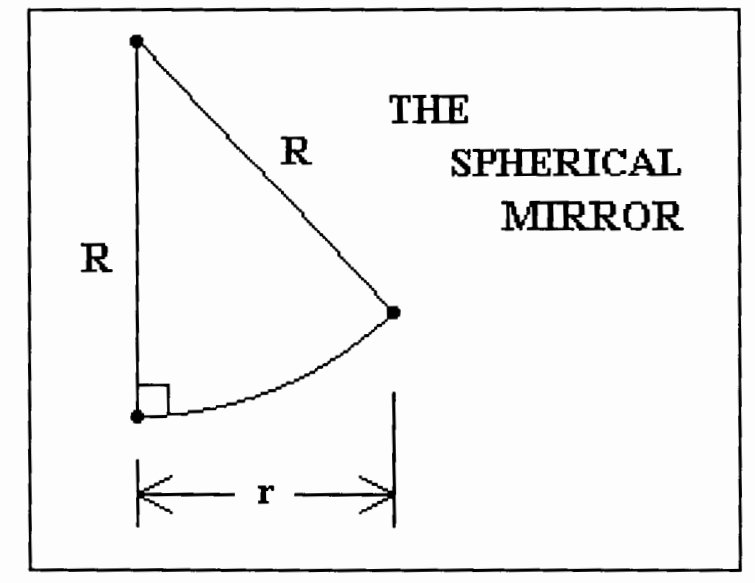

Figure 15. Critical points of the Spherical Mirror.

Here $\mathrm{r}$ is the mirror radius, $\mathrm{d}$ is the mirror separation or cavity length and $\lambda$ is the wavelength of the laser light.

\section{THE FRESNEL MIRROR}

The Fresnel Mirror is essentially sections of a Spherical Mirror that are set side by side in a semi-planar manner as shown in Figure 16. Imagine drawing some concentric circles spaced $\lambda / 2$ apart. Then draw a line tangent to one of the circles. The intersection of the line with the circles defines the planar direction of the mirror. Next we draw two parallel dashed lines a quarter of a wavelength above and below the solid line. Then the mirror is defined by tracing each circle between the dashed lines as shown in Figure 16 (The scale of Figures 13-15 is greatly skewed. In practice, the mirror curvature $\mathrm{R}$ is on the order of one meter whereas the mirror radius $r$ is on the order of ten millimeters).

In actuality, the transition between circles is almost a vertical transition. Note that the transition distance is purposely selected to be a half a wavelength. Two reflected waves, one on each side of the transition, will have a phase difference equal to $2 \pi$, i.e. a wavelength. This is because one of the waves travels a half wavelength farther before being reflected as well as a half wavelength farther after the reflection. Thus this wave 


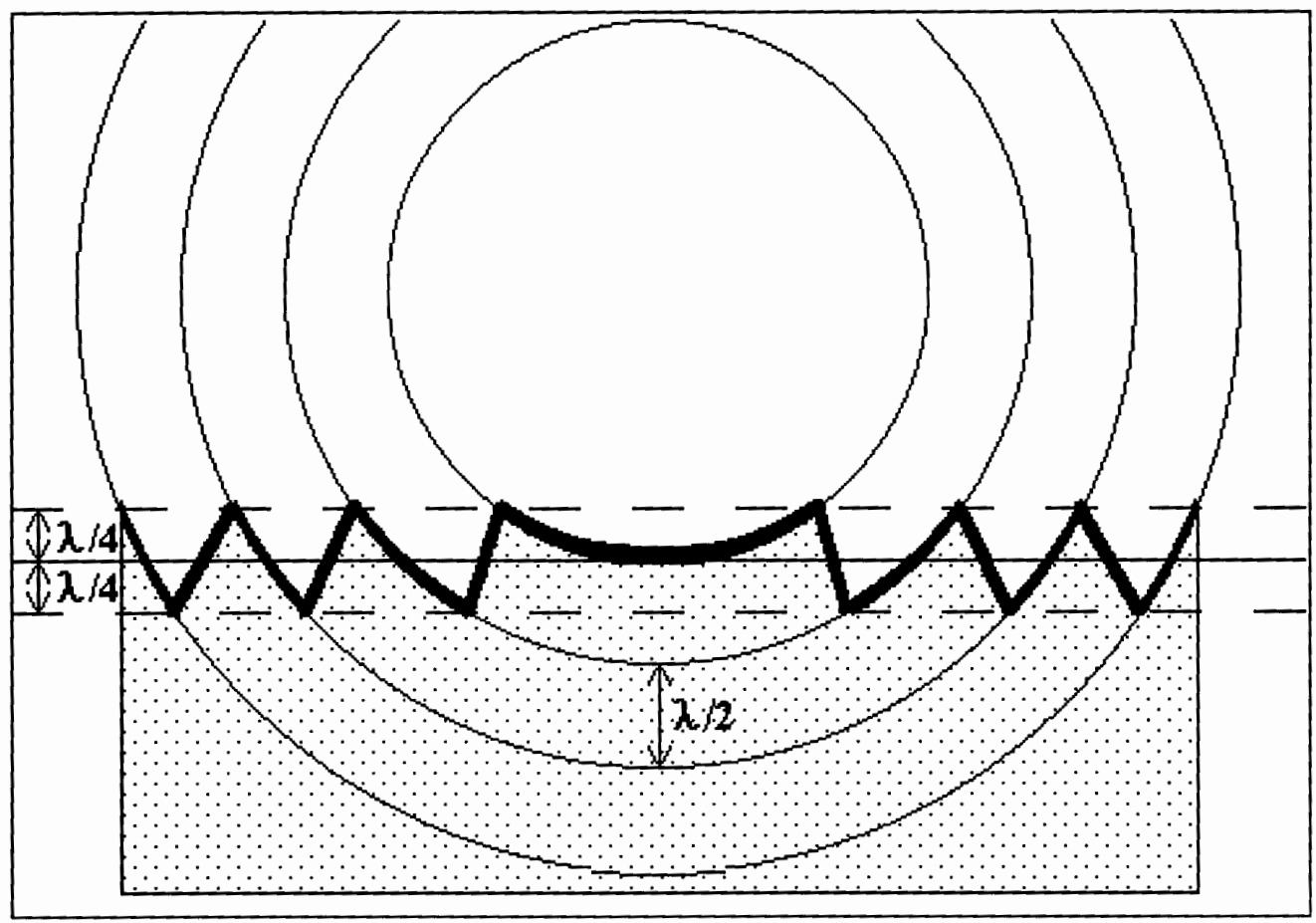

Figure 16. A cross-section of a Fresnel Mirror.

will lag the other in phase by $2 \pi$. Figure 17 shows the critical points in the design of the Fresnel Mirror.

\section{The Fresnel Number $\mathrm{N}$}

The Fresnel number is an important number in the discussion of resonator mirrors with circular symmetry. It accounts for the mirror size which affects the diffraction loss or beam spill over at the edge and also the number of TEM modes allowed to oscillate without being quenched by diffraction losses. It is derived as follows [16].

Assume a plane wave is originated from a circular aperture as shown in Figure 18. The wave front is divided into a number of annular regions called Fresnel zones such that the boundaries are increments of $\lambda / 2$. The Fresnel zone radii are defined from the figure as

$$
\mathrm{r}_{\mathrm{N}}^{2}=(\mathrm{d}+\mathrm{N} \lambda / 2)^{2}-\mathrm{d}^{2} \text { or } \mathrm{r}_{\mathrm{N}}^{2}=\mathrm{Nd} \lambda+(\mathrm{N} \lambda / 2)^{2}
$$




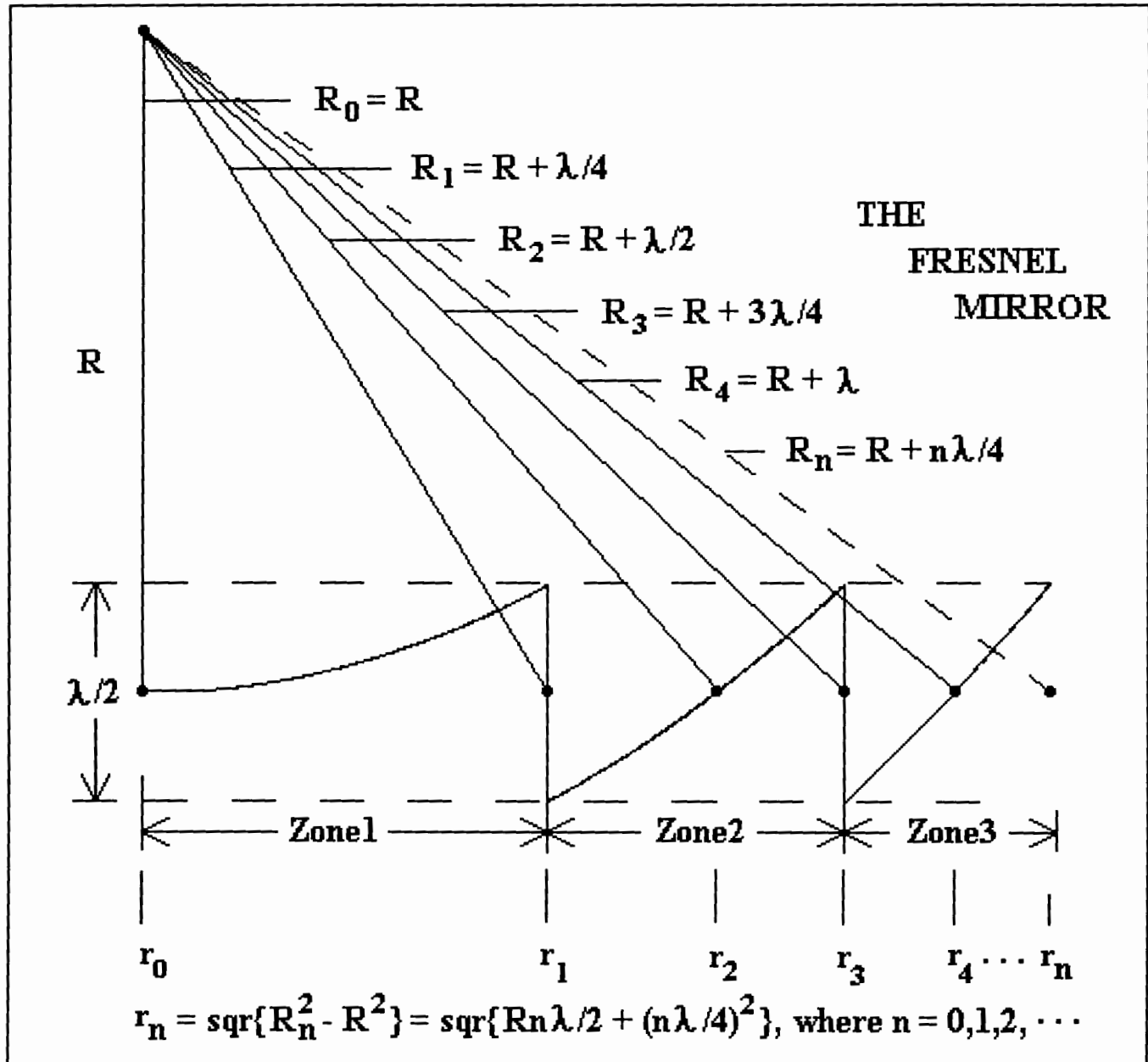

Figure 17. Critical points of the Fresnel Mirror. Shown is a radial plot of a Fresnel Mirror whose size spans the first three Fresnel zones.

With $d \gg>\lambda$ and $N$ not being extremely large, we can neglect the second term. Thus

$$
r_{N}^{2}=N \lambda d \Rightarrow N=r_{N}{ }^{2} / \lambda d \text {. }
$$

Here $\mathrm{N}$ is an integer yet in general $\mathrm{N}$ is a positive number.

We now ask the question, how many Fresnel zones are encompassed by the circular aperture? From the right triangle indicated by AOP in Figure 18, we have

$$
N=r_{N}^{2} / \lambda d
$$

where $N=$ the number of Fresnel zones within the aperture as seen from point $P$. 


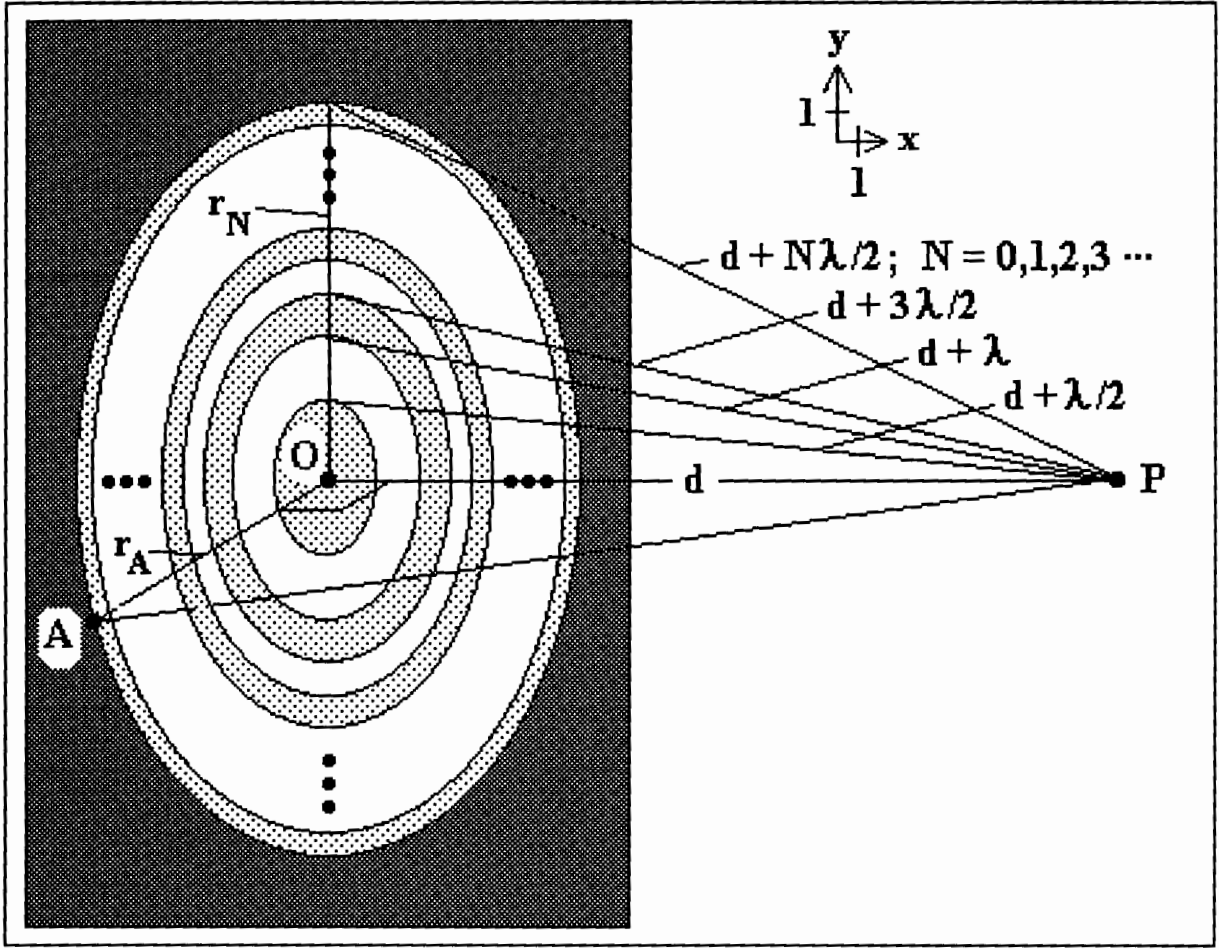

Figure 18. A circular aperture with a transmitted plane wave divided into annular rings. Each ring is a Fresnel zone with $\mathrm{N}$ equalling the Fresnel number.

The intensity at point $\mathrm{P}$ will rise periodically from zero to a maximum and back again to a minimum as the number of Fresnel zones within the aperture is increased from zero. This is because successive Fresnel zones tend to cancel each other. The resultant phase angle when radially traversing any Fresnel zone is equal to $\pi$ radians.

For each additional Fresnel zone the vibration curve rotates one-half turn and a phase angle of $\pi$ as it spirals inward. Thus when traversing any two adjacent zones, the resultant phase angle equals zero radians yet the resultant amplitude does not quite equal zero due to the obliquity factor.

If we replace the circular aperture with a reflective mirror, one can change the phase difference between odd and even zones so that both sets are in phase with each other as seen at the point $P$. This is accomplished by having a step height difference between the odd and even zones equal to $\lambda / 4$. Note that the phase is not constant 
throughout any zone due to the planar structure. Ideally the phase should not change as in the case of the Spherical Mirror[17].

\section{THE TIERED FRESNEL MIRROR}

The Tiered Fresnel Mirror is an approximated version of the Fresnel Mirror. The degree of approximation is directly proportional to the number of tiers per zone, where a tier is of constant step height and a zone is a group of tiers. The first zone covers the first half of the first Fresnel zone. The second zone covers the second half of the first Fresnel zone to the first half of the next Fresnel zone and all succeeding zones follow the pattern of the second zone. This is indicated in Figure 19. Also shown are the tier radii $r_{\mathrm{Z}}$. These are phase matched such that each tier covers the same phasor angle in the vibration curve equal to $\pi /($ tiers_per_zone) radians.

\section{DIFFRACTION LOSSES}

As the mirror radius increases, more higher order transverse modes begin to oscillate since the peak of the outermost ripple of each mode extends outward in the radial direction. To illustrate this Figure 20 shows the first three Laguerre-Gaussian $\mathrm{TEM}_{\mathrm{p} 0}$ transverse modes.

The mode half-width $x_{p}$ is defined as the peak of the outermost ripple of the Laguerre-Gaussian pattern [18]. This half-width or spread is proportional to the radial index $\mathrm{p}$ in approximately the form

$$
x_{p} \approx(p)^{1 / 2} w
$$

The number of transverse modes that will fit within the mirror radius $r$ is given by the radial index $p$ so that

$$
x_{p} \leq r \text { or }
$$




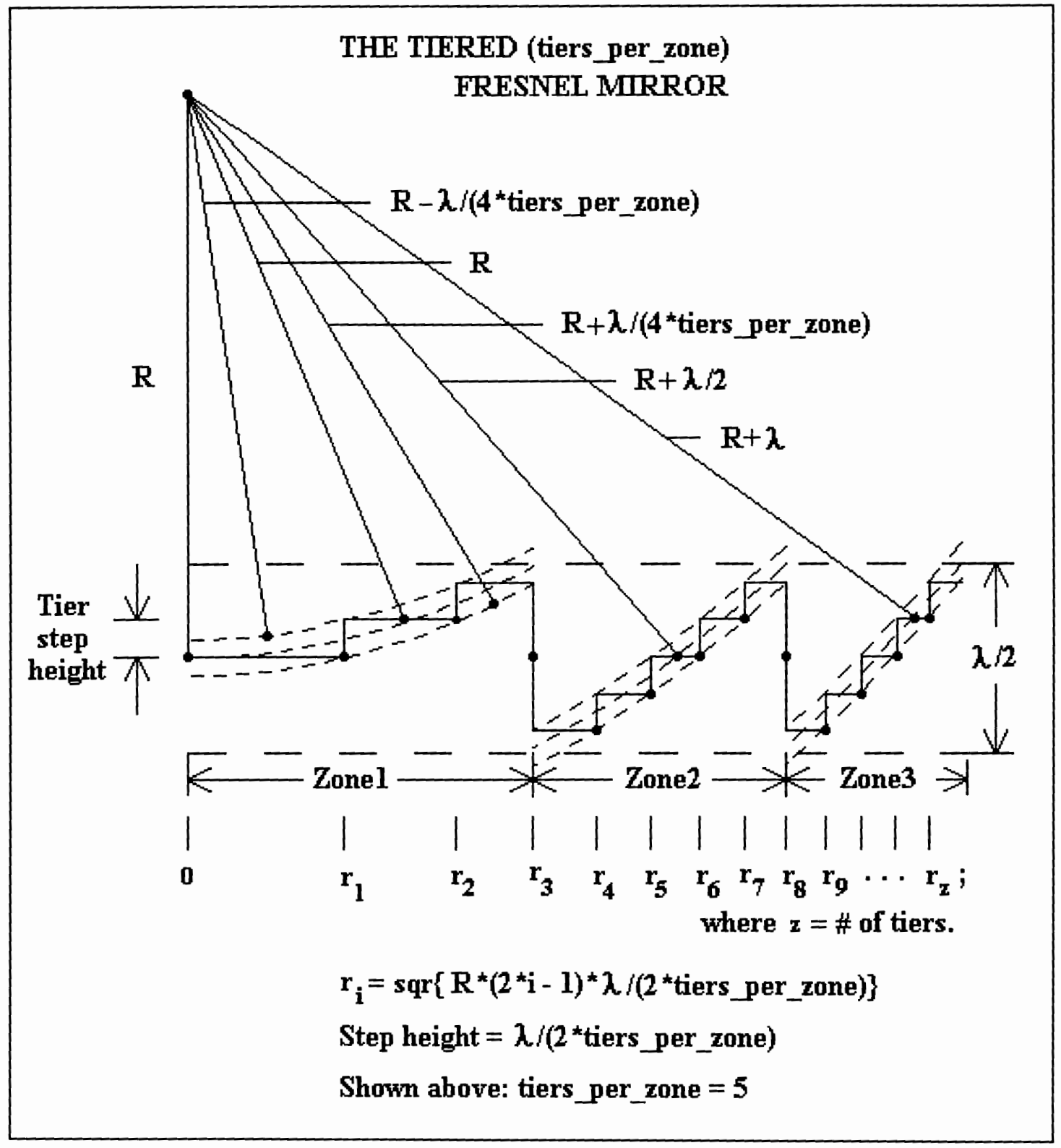

Figure 19. Critical points of the Tiered Fresnel Mirror. The dotted lines are the edge boundaries of the tiers. Each tier spans an equal phase.

$$
w(p)^{1 / 2} \leq r \Rightarrow p \leq r^{2} / w^{2} .
$$

For the symmetric confocal resonator $\mathrm{w}^{2}=\lambda \mathrm{d} / \pi$ and using the Fresnel Number $\mathrm{N}$ we have

$$
\mathrm{p} \leq \mathrm{r}^{2} / \mathrm{w}^{2} \Rightarrow \mathrm{p} \leq \pi \mathrm{N} .
$$




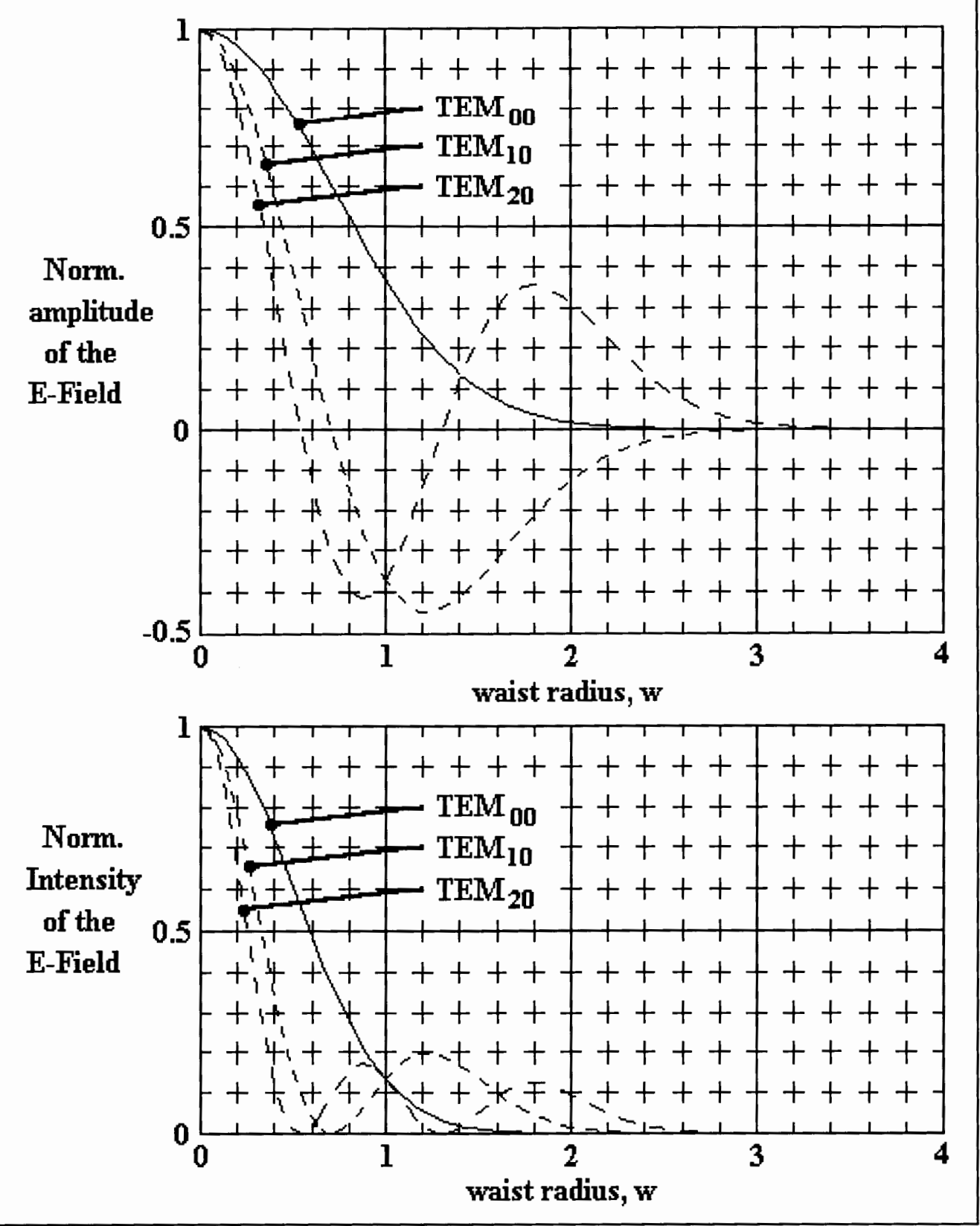

Figure 20. The first three Laguerre-Gaussian $\mathrm{TEM}_{\mathrm{p} 0}$ modes vs. the waist radius. The modes extend more radially with increasing mode index $\mathrm{p}$.

For example, if the mirror radius equals the waist at the mirror then both $\mathrm{TEM}_{00}$ and $\mathrm{TEM}_{10}$ modes will fit within the mirror and higher modes will spill over the mirror's edge and will quickly die out due to diffraction. Figure 20 indicates, that when r equals $\mathrm{w}$, the $\mathrm{TEM}_{10}$ mode is going to be attenuated much more rapidly than the $\mathrm{TEM}_{00}$ mode 
since a major portion of the $\mathrm{TEM}_{10}$ mode's spatial extent is truncated by the mirror's edge.

As the mirror radius increases, more TEM modes will oscillate in a low loss condition. Notice that the $\mathrm{TEM}_{00}$ mode at any mirror radius has the least loss per transit or pass of any of the TEM modes. Thus the term dominant or fundamental mode is commonly referred to the $\mathrm{TEM}_{00}$ mode. The term transit refers to the beam's traverse from one mirror to the other.

\section{The Fox and Li Method}

In 1961, when the laser was in its infancy, A.G. Fox and Tingye Li wrote a classical paper investigating diffraction effects in symmetric laser resonators[19]. They introduced a method to determine the steady state Electric Field or E-Field from an initially launched wave within the resonator. The method they used is still valid and used today. The Fox and Li method is used to study the diffraction loss in a variety of different mirrors such as a hole in the center or a mirror consisting of annular rings. Any arbitrary mirror shape can be analyzed as long as the physical dimensions of the mirrors are accurately known. This includes sensitivity studies where the effects of mirror imperfections can be modeled.

The method used is as follows: 1) an initial arbitrary wave is launched from one mirror, say $\mathrm{M}_{2}$, towards the other mirror $\mathrm{M}_{1} ; 2$ ) the E-Field distribution at $\mathrm{M}_{1}$; is computed by use of the Huygens-Fresnel diffraction integral evaluated at $\mathrm{M}_{2} ; 3$ ) the wave is then reflected from $\mathbf{M}_{1}$ and the new E-Field at $\mathbf{M}_{2}$ is re-computed in similar manner from the calculated E-Field at $\mathrm{M}_{1}$; and 4) the computation of the E-Field distribution is repeated over and over again for subsequent successive transits of the transformed wave until a steady state is reached. 
This iterative method is analogous to the physical process involved in the resonator when the laser beam is first initiated by noise or the spontaneous emission of the laser medium.

Symmetric Resonators. We now will apply the Fox and Li method to determine the steady state E-Field distribution in a symmetric resonator.

If the mirror radius is large compared to the wavelength, the E-Field is very nearly transverse in spatial extent, and the E-Field is uniformly polarized in one dimension then the scalar form of the Huygens-Fresnel diffraction integral can be used [20]. The E-Field due to the illuminated aperture $\mathrm{A}$ is given by

$$
\mathrm{E}_{\mathrm{qpm}}=\frac{i}{2 \lambda} \int_{\mathrm{A}} \mathrm{E}_{(\mathrm{q}-1) \mathrm{pm}} \frac{e^{-i \mathrm{kD}}}{\mathrm{D}}(1+\cos (\theta)) \mathrm{dS},
$$

where $\mathrm{k}$ is the propagation constant of the medium; $\mathrm{D}$ is the distance from a point on the aperture to the point of observation; $\theta$ is the angle that $\mathrm{D}$ makes with the unit normal to the aperture; $q$ is the number of transits that the beam makes; $\mathrm{E}_{0 \mathrm{pm}}$ is the initial wave launched from the aperture $M_{2}$; the aperture $A$ is either $M_{1}$ if $q$ is even or $M_{2}$ when $q$ is odd; and pm are the radial and azimuthal Laguerre-Gaussian TEM mode indices.

After many q transits the initial E-Field will eventually reach a steady state. This is when the E-Fields at each mirror differ only by a complex constant. Thus we can write

$$
\mathrm{E}_{\mathrm{qpm}}=\left(\gamma_{\mathrm{ST}}\right)_{\mathrm{pm}}{ }^{-\mathrm{q}} \mathrm{v}_{\mathrm{pm}}=\exp \left[-\mathrm{q} \ln \left(\gamma_{\mathrm{ST}}\right)_{\mathrm{pm}}\right] \mathrm{v}_{\mathrm{pm}},
$$

where $\mathrm{v}_{\mathrm{pm}}$ is a constant distribution function, $\left(\gamma_{\mathrm{ST}}\right)_{\mathrm{pm}}$ is a complex constant independent of position coordinates and the ST denotes a single transit.

The logarithm of $\left(\gamma_{\mathrm{ST}}\right)_{\mathrm{pm}}$ is the single transit propagation constant associated with the normal mode corresponding to a steady-state solution and specifies the attenuation and phase shift that the wave suffers during each transit.

When we substitute $\left(\gamma_{S T}\right)_{p m}{ }^{-q} v_{p m}$ for $E_{q p m}$ and $\left(\gamma_{S T}\right)_{p m}{ }^{-(q-1)} v_{p m}$ for $E_{(q-1) p m}$ in the diffraction integral we obtain the integral equation 


$$
\mathrm{v}_{\mathrm{pm}}=\left(\gamma_{\mathrm{ST}}\right)_{\mathrm{pm}} \int_{\mathrm{A}} \mathrm{v}_{\mathrm{pm}} \mathrm{K}_{\mathrm{A}} \mathrm{dS} \mathrm{S}_{\mathrm{A}}
$$

where $\mathrm{K}_{\mathrm{A}}=(i / 2 \lambda \mathrm{D})(1+\cos (\theta)) e^{-i \mathrm{kD}}=$ the kernel of the integral equation, and $\left(\gamma_{\mathrm{ST}}\right)_{\mathrm{pm}}$ is the eigenvalue to the eigensolution $\mathrm{v}_{\mathrm{pm}}$ of the integral equation. The distribution function $\mathrm{v}_{\mathrm{pm}}$, which satisfies the integral equation, is the normal mode of the symmetric resonator defined at the mirror surface.

The ratio of $E_{(q+1) p m}$ to $E_{q p m}$ is less than one due to spill over of the beam at the mirror edge caused by diffraction. This is given by

$$
\frac{\mathrm{E}_{(q+1) p m}}{E_{\mathrm{qpm}}}=\frac{\left(\gamma_{S T}\right)_{\mathrm{pm}}{ }^{-(q+1)} v_{\mathrm{pm}}}{\left(\gamma_{S T}\right)_{\mathrm{pm}}{ }^{-q} v_{\mathrm{pm}}}=\left(\gamma_{\mathrm{ST}}\right)_{\mathrm{pm}}{ }^{-1}
$$

The fractional power loss at the mirrors is given in Table I.

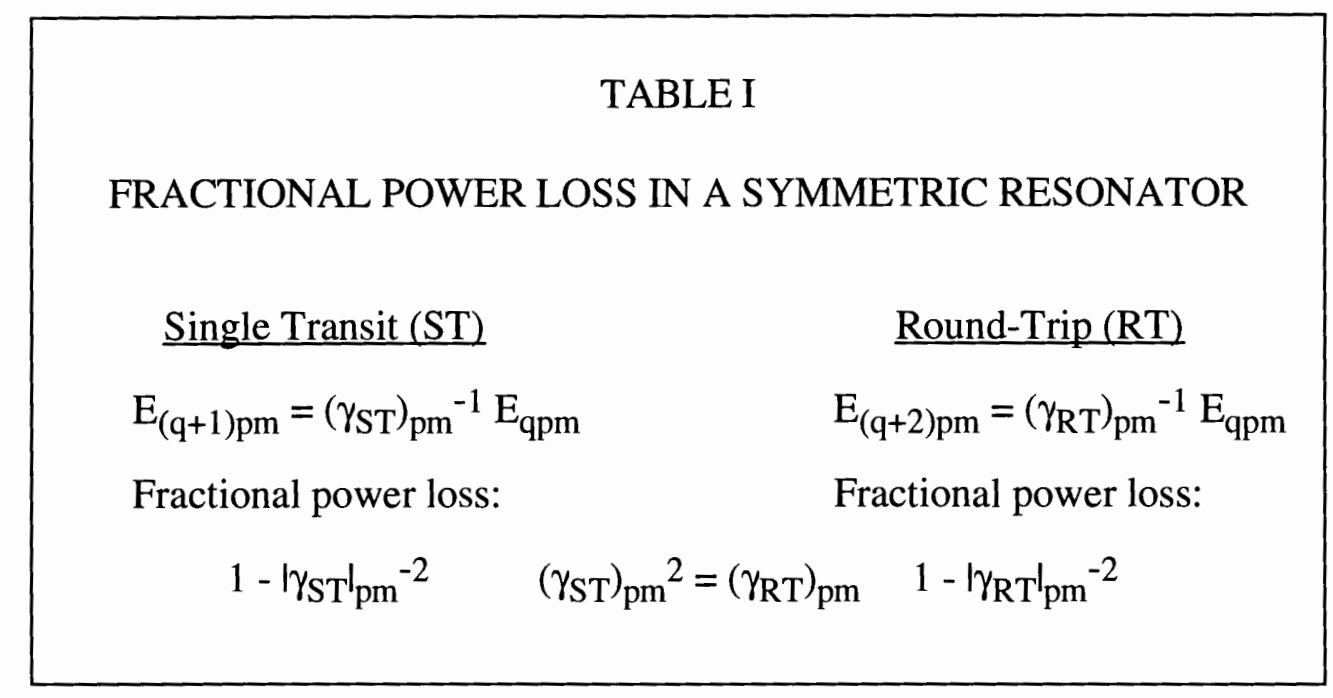

Non-Symmetric Resonators. We will now apply the Fox and Li method to a nonsymmetric resonator. A round-trip must be studied since no eigenvalues of the integral equation exist for the single pass. This is because the different mirrors cause the E-Fields at each mirror to be different in spatial extent during the steady state condition. We will end up with a double integral equation since integration is performed over each mirror. 
This round-trip analysis can be considered as general since a symmetric resonator can be analyzed by using this technique.

We begin with the diffraction integral with an initial wave $E_{0 p m}$ propagating one transit from mirror $\mathrm{M}_{2}$ in the form

$$
\mathrm{E}_{1 \mathrm{pm}}=\frac{i}{2 \lambda} \int_{\mathrm{M}_{2}} \mathrm{E}_{0 \mathrm{pm}} \frac{e^{-i \mathrm{kD}} 2}{\mathrm{D}_{2}}\left(1+\cos \left(\theta_{2}\right)\right) \mathrm{d} \mathrm{S}_{2} .
$$

After a reflection at $\mathrm{M}_{1}$, the beam completes a round-trip by making another transit and we obtain

$$
\mathrm{E}_{2 \mathrm{pm}}=\frac{i}{2 \lambda} \int_{\mathrm{M}_{1}} \mathrm{E}_{1 \mathrm{pm}} \frac{e^{-i \mathrm{kD}}}{\mathrm{D}_{1}}\left(1+\cos \left(\theta_{1}\right)\right) \mathrm{d} \mathrm{S}_{1} .
$$

By substitution of $E_{1}$ into the equation for $E_{2}$ we have

$$
E_{2 p m}=\int_{M_{1} M_{2}} E_{0 p m} K_{1} K_{2} d S_{2} d S_{1}
$$

where $K_{j}=\left(i / 2 \lambda D_{j}\right)\left(1+\cos \left(\theta_{j}\right)\right) e^{-i k D_{j}} ;(j=1,2)$ is the kernel of the double integral equation.

After many t round-trips a steady state evolves. The rate of convergence is a function of: 1) the form of the input wave $\mathrm{E}_{0 \mathrm{pm}}$; and 2) the Fresnel number N. Again we describe the E-Field after $t$ round-trips as

$$
E_{t p m}=\left(\gamma_{R T}\right)_{p m}{ }^{-t} v_{p m}
$$

where the subscript RT denotes a round-trip and again $v_{p m}$ is a constant distribution function and $\left(\gamma_{\mathrm{RT}}\right)_{\mathrm{pm}}$ is a complex constant independent of position coordinates. The logarithm of $\left(\gamma_{\mathrm{RT}}\right)_{\mathrm{pm}}$ is the round-trip propagation constant associated with the normal mode and specifies the attenuation and phase shift that the wave suffers during each round-trip.

When we substitute $\left(\gamma_{\mathrm{RT}}\right)_{\mathrm{pm}}{ }^{-\mathrm{t}} \mathrm{v}_{\mathrm{pm}}$ for $\mathrm{E}_{2 \mathrm{pm}}$ and $\left(\gamma_{\mathrm{RT}}\right)_{\mathrm{pm}}{ }^{-(\mathrm{t}-1)} \mathrm{v}_{\mathrm{pm}}$ for $\mathrm{E}_{0 \mathrm{pm}}$ in the diffraction integral, we obtain the double integral equation 


$$
v_{\mathrm{pm}}=\left(\gamma_{\mathrm{RT}}\right)_{\mathrm{pm}} \int_{\mathrm{M}_{1} \mathrm{M}_{2}} \mathrm{v}_{\mathrm{pm}} \mathrm{K}_{1} \mathrm{~K}_{2} \mathrm{dS}_{2} \mathrm{dS} \mathrm{S}_{1}
$$

The ratio of the E-Fields at each mirror within a round-trip is meaningless since each has different spatial extents, while $E_{(t+1) p m}$ to $E_{t p m}$ specifies the attenuated field given by

$$
\frac{E_{(t+1) p m}}{E_{t p m}}=\frac{\left(\gamma_{R T}\right)_{p m}-(t+1) v_{p m}}{\left(\gamma_{R T}\right)_{p m}^{-t} v_{p m}}=\left(\gamma_{R T}\right)_{p m}^{-1}
$$

The fractional power loss per round-trip is given in Table II.

\section{TABLE II}

FRACTIONAL POWER LOSS IN A NON-SYMMETRIC RESONATOR

Single Transit (ST)

$\mathrm{E}_{(\mathrm{t}+1 / 2) \mathrm{pm}} \neq\left(\gamma_{\mathrm{RT}}\right)_{\mathrm{pm}}{ }^{-1 / 2} \mathrm{E}_{\mathrm{tpm}}$

Fractional power loss:

\section{Round-Trip(RT)}

$\mathrm{E}_{(\mathrm{t}+1) \mathrm{pm}}=\left(\gamma_{\mathrm{RT}}\right)_{\mathrm{pm}}{ }^{-1} \mathrm{E}_{\mathrm{tpm}}$

Fractional power loss:

$$
\text { undefined } \quad\left(\gamma_{\mathrm{ST}}\right)_{\mathrm{pm}} \text { is undefined } \quad 1-\left|\gamma_{\mathrm{RT}}\right|_{\mathrm{pm}}{ }^{-2}
$$

To be thorough when discussing the losses in a resonator we must include all Laguerre-Gaussian TEM modes. Any transverse wave can be decomposed into a complete set of modes.

For example, the input wave specified by $\mathrm{E}_{0}$ is actually a composite of LaguerreGaussian modes, that by superposition, comprise $\mathrm{E}_{0}$ as

$$
\mathrm{E}_{0}(\mathrm{r}, \varphi)=\sum_{\mathrm{pm}} \mathrm{c}_{\mathrm{pm}} \mathrm{E}_{\mathrm{pm}}(\mathrm{r}, \varphi)
$$

Each transverse mode in the integral equation has its own eigenvalue: $\left(\gamma_{\mathrm{ST}}\right)_{\mathrm{pm}}$ for a symmetrical resonator; and $\left(\gamma_{\mathrm{RT}}\right)_{\mathrm{pm}}$ for a non-symmetric resonator. 
In a symmetrical resonator, when a steady state is achieved after q transits, the EField at the mirror can be written as

$$
E^{[q]}(r, \varphi)=\sum_{p m} c_{p m}\left(\gamma_{S T}\right)_{p m}-q E_{p m}(r, \varphi)
$$

The relative amplitude of each transverse mode after $q$ transits will in general be different and will be exponentially attenuated by $\gamma_{S T} \mathrm{pm}^{\mathrm{q}}$. The TEM 00 mode will have the largest eigenvalue or lowest loss per transit. All other modes have smaller eigenvalues and thus will die out quicker at different rates depending on the value of their particular eigenvalue.

To regress, we rewrite the integral equations for a symmetric and a non-symmetric resonator as

$$
\begin{gathered}
\mathrm{v}_{\mathrm{pm}}=\left(\gamma_{\mathrm{ST}}\right)_{\mathrm{pm}} \int_{\mathrm{A}} \mathrm{v}_{\mathrm{pm}} \mathrm{K}_{\mathrm{A}} \mathrm{d} \mathrm{S}_{\mathrm{A}} \\
\text { and } \\
\mathrm{v}_{\mathrm{pm}}=\left(\gamma_{\mathrm{RT}}\right)_{\mathrm{pm}} \int_{\mathrm{M}_{1} \mathrm{M}_{2}} \mathrm{v}_{\mathrm{pm}} \mathrm{K}_{1} \mathrm{~K}_{2} \mathrm{dS}_{2} \mathrm{~d} S_{1}
\end{gathered}
$$

respectively. 


\section{CHAPTER IV}

\section{COMPUTER SIMULATIONS}

For the study of Tiered Fresnel Mirrors, we will use only circular symmetric mirrors and a Laguerre-Gaussian beam where the azimuthal index m equals zero. We will work in cylindrical coordinates with the set-up shown in Figure 21.

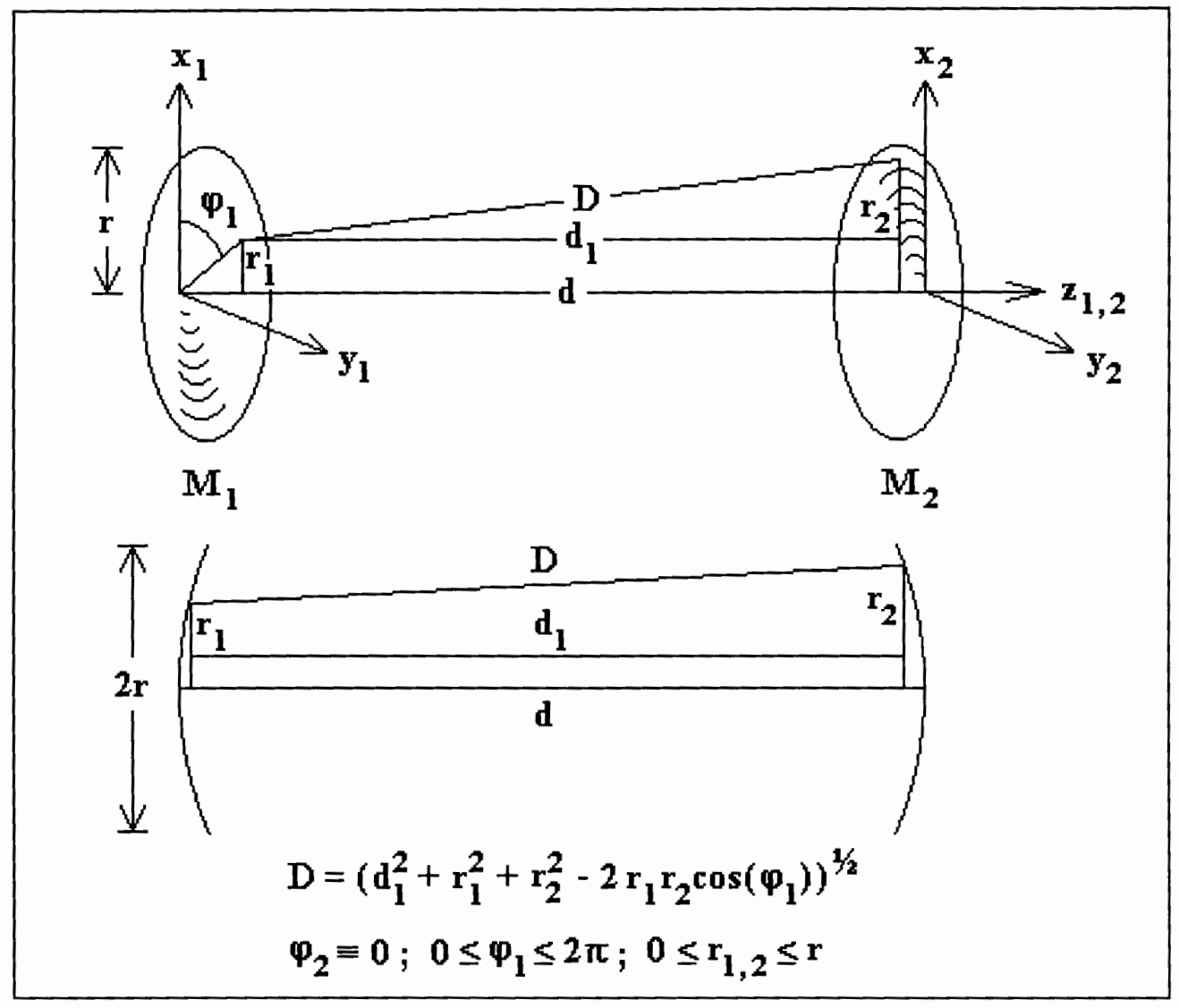

Figure 21. Geometry of a Symmetric Resonator. 
The Fox and Li method is used where, at the observation mirror, a radial line at $\varphi=0$ is taken and the E-Field is determined at equal incremented points from the mirror center to its edge along this radial line. Since cylindrical symmetry exists, the field along this radial line can be rotated from 0 to $2 \pi$ in equal increments, and duplicated to fill the observation mirror with the observed E-Field distribution.

This process is replicated at the other mirror due to the beam's reflection and iterated back and forth until the E-Field varies negligibly in three consecutive transits.

We begin with the Huygens-Fresnel diffraction integral where once again stated is

$$
\mathrm{E}_{\mathrm{qpm}}=\frac{i}{2 \lambda} \int_{\mathrm{A}} \mathrm{E}_{(\mathrm{q}-1) \mathrm{pm}} \frac{e^{-i \mathrm{kD}}}{\mathrm{D}}(1+\cos (\theta)) \mathrm{dS},
$$

This integral can be separated into two integrals since both $\mathrm{E}_{\mathrm{qpm}}$ and $\mathrm{E}_{(\mathrm{q}-1) \mathrm{pm}}$ have real and imaginary components.

We can set $\cos (\theta)=d_{1} / D$ since the mirror sizes are small compared to the spacing $d\left(d_{1}\right.$ is shown in Figure 21). We will also replace the following: $E_{q p m}$ by $\left(E_{q p m}\right)_{R}+$ $i\left(\mathrm{E}_{\mathrm{qpm}}\right)_{\mathrm{I}} ; \mathrm{E}_{(\mathrm{q}-1) \mathrm{pm}}$ by $\left(\mathrm{E}_{(\mathrm{q}-1) \mathrm{pm}}\right)_{\mathrm{R}}+i\left(\mathrm{E}_{(\mathrm{q}-1) \mathrm{pm}}\right)_{\mathrm{I}} ; e^{-i \mathrm{kD}}$ by $[\cos (\mathrm{kD})-i \sin (\mathrm{kD})] ;$ and $\mathrm{dS}$ by $\rho d \rho d \varphi$. After some manipulation we obtain the real and imaginary parts of the E-Field as

$\left(E_{\mathrm{qpm}}\right)_{\mathrm{R}}=\int_{0}^{2 \pi} \frac{1}{2 \lambda} \int_{0}^{\mathrm{r}} \frac{\left(1+\mathrm{d}_{1} / \mathrm{D}\right)}{\mathrm{D}}\left[\left(\mathrm{E}_{(\mathrm{q}-1) \mathrm{pm}}\right)_{\mathrm{R}} \cos (\mathrm{kD})-\left(\mathrm{E}_{(\mathrm{q}-1) \mathrm{pm}}\right)_{\mathrm{I}} \sin (\mathrm{kD})\right] \rho \mathrm{d} \rho \mathrm{d} \varphi$

and

$\left(E_{\mathrm{qpm}}\right)_{\mathrm{I}}=\int_{0}^{2 \pi} \frac{1}{2 \lambda} \int_{0}^{\mathrm{r}} \frac{\left(1+\mathrm{d}_{1} / \mathrm{D}\right)}{\mathrm{D}}\left[\left(\mathrm{E}_{(\mathrm{q}-1) \mathrm{pm}}\right)_{\mathrm{R}} \sin (\mathrm{kD})+\left(\mathrm{E}_{(\mathrm{q}-1) \mathrm{pm}}\right)_{\mathrm{I}} \cos (\mathrm{kD})\right] \operatorname{\rho d} \rho \mathrm{d} \varphi$.

These two equations are iterated with each transit that the beam makes until a steady state solution of the E-Field distribution is found. 
A computer program was written to determine the loss per pass (or performance) of various resonator types and in particular to compare the performance of the novel Tiered Fresnel Mirror to that of the common Spherical Mirror. The program is listed in Appendix A along with a cross reference mapping of all the variables used which is found in Appendix B.

An example output of a short computer run having only five round-trips is shown in Figure 22. Notice that the input wave is a plane wave and already it has been drastically transformed from a horizontal line equal to 1 for the normalized amplitude to that of the solid curve in sections $\mathrm{U}$ and $\mathrm{V}$ in Figure 22.

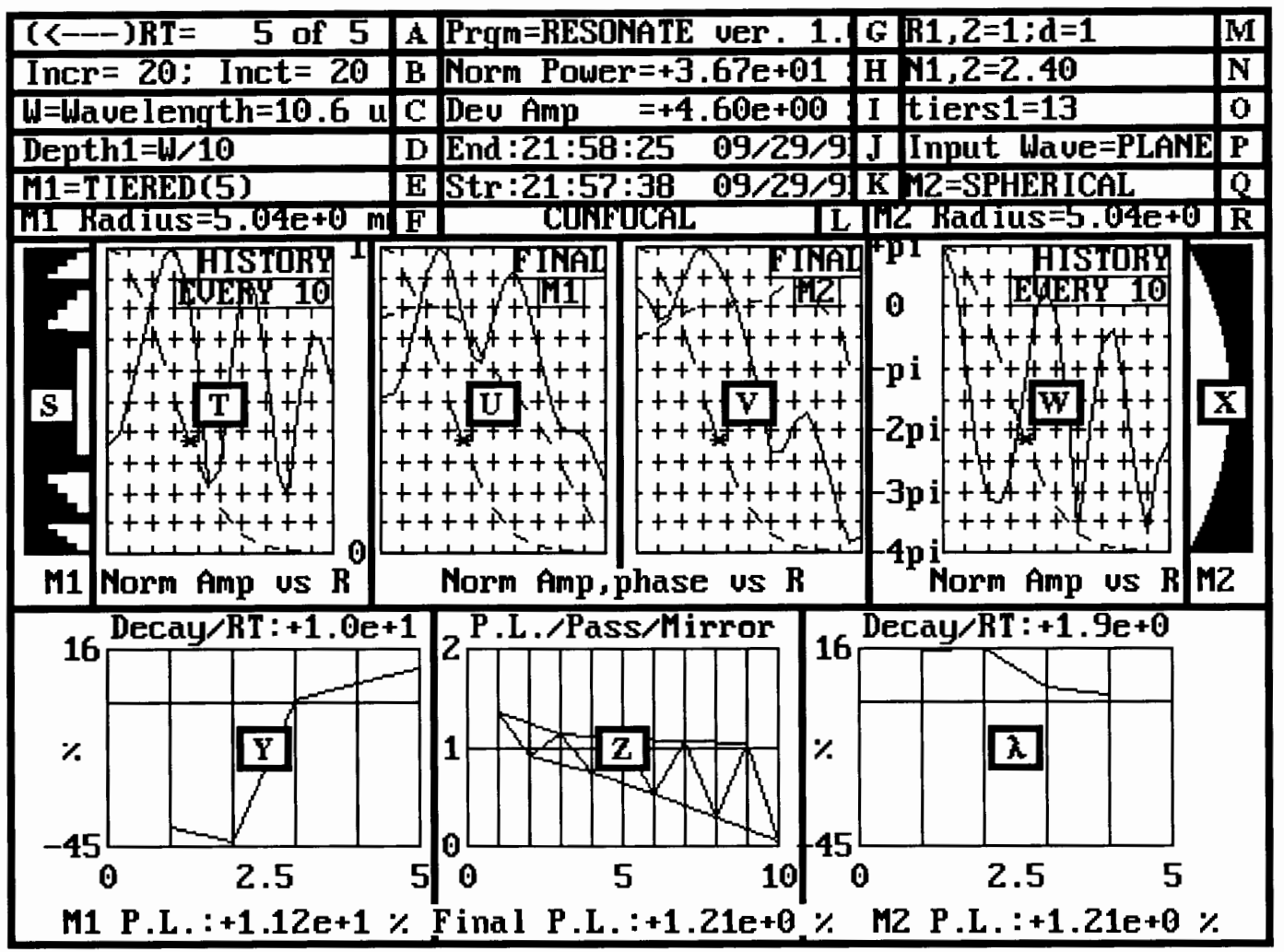

Figure 22. An example output of a computer simulation of a Tiered(5) Fresnel Mirror paired with a Spherical Mirror. 
Each section of the figure is labeled with a letter A-Z and $\lambda$. The explanation of each section is as follows:

A) The arrow indicates the direction the beam travels. "RT" stands for roundtrips.

B) "Incr" is the number of radial increments and "Inct" is the number of azimuthal increments used in the integration of the mirror.

C) "W=Wavelength" is the wavelength of the oscillating beam.

D) "Depth1,2" indicates the etch depth or step height of the tier on mirrors $M_{1}$ and $\mathrm{M}_{2}$. Displayed only for Tiered Fresnel Mirrors.

E) "M1" is the mirror on the left. The available mirror types for $\mathrm{M}_{1}$ and $\mathrm{M}_{2}$ are Spherical, Parabolic, Plane, Tiered and Fresnel. For the Spherical type one can use either positive or negative mirror curvatures. For the Tiered Fresnel Mirror one can use any odd number of tiers per zone. This number is denoted within the parentheses.

F) "M1 Radius" is the radial dimension of mirror $\mathbf{M}_{1}$.

G) $" P r g m=$ RESONATE ver 1.0" is the program name and current version.

H) "Norm Power" is the current power illuminated on the proper mirror relative to the power of the input beam. This value decreases with each reflection of the beam due to beam spill over at the mirror's edge.

I) "Dev Amp" is the deviation or change in area under the amplitude curve relative to the last calculated area of the particular mirror. In other words the value 1 - $\left(d e v \_a m p_{q} / d e v_{-} a m p_{q-1}\right)$, where $q$ is the transit number. This value is evaluated at $M_{1}$ if $q$ is even or at $M_{2}$ if $q$ is odd. If three consecutive round-trips of the beam all yield dev_amp values of less than $7 e-4 \%$ then the program terminates and it is assumed that a steady state has been reached. 
NOTE: The program actually plots the square root of the intensity yielding curves in the positive domain. Hereafter use of the term amplitude pertaining to the program will actually be the square root of the intensity.

J) "End" indicates the time that the program stops.

K) "Str" indicates the time that the program starts.

L) "CONFOCAL" is the special resonator type. The types displayed are dependent upon the system parameters. The available resonator types are Plane-Parallel, Half Confocal, Half Concentric, Confocal, Concentric, and for non-special resonators; Confined Beam or Unconfined Beam.

M) "R1,2" indicates the radius of mirror curvature of mirrors $M_{1}$ and $M_{2}$. This value is variable for the Spherical Mirror and fixed at one meter for both Tiered and Fresnel Mirrors. If the mirror is Parabolic the value displayed is PARAB or if the mirror is Plane then PLANE is displayed. " $\mathrm{d}$ " is the cavity length or mirror separation.

$\mathrm{N})$ "N1,2" is the Fresnel number of mirrors $\mathrm{M}_{1}$ and $\mathrm{M}_{2}$. This is the number of Fresnel zones that span a mirror as seen from the center of the other mirror.

O) "tiers1,2" is the total number of tiers of mirrors $\mathrm{M}_{1}$ and $\mathrm{M}_{2}$. Displayed only for Tiered Fresnel Mirrors.

P) "Input Wave" is the form of the initial wave. A plane or Gaussian wave are the available choices.

Q) "M2" is the mirror type shown on the right side.

R) "M2 Radius" is the radial dimension of mirror $\mathbf{M}_{2}$.

S) This is an illustration of mirror $\mathrm{M}_{1}$.

T) This graph is a history of mirror $\mathbf{M}_{1}$ 's normalized amplitude vs. the mirror radius. Every 10th round-trip the E-Field distribution is plotted, i.e. 1, 
$11,21,31, \cdots$ round-trips are plotted. In this section (as well as sections $\mathrm{U}$, $\mathrm{V}$, and $\mathrm{W}$ ) the following pertains: 1) the horizontal axis is the mirror radius where the center $(r=0)$ is located at the left and the mirror's edge $\left(r=r_{\max }\right)$ is on the right;2) the vertical axis is the normalized amplitude with the value of one at the top and zero at the bottom; 3) a dashed Gaussian curve is the theoretical E-Field distribution of the Laguerre-Gaussian $\mathrm{TEM}_{00}$ mode independent of the mirror radius; and 4) an asterisk on the dotted Gaussian curve that indicates the $1 / e$ point which is the waist radius at the mirror.

$\mathrm{U}$ ) This graph is mirror $\mathrm{M}_{1}$ 's final E-Field distribution (the solid curve) and a plot of the phase of the final E-Field both vs. the mirror radius. In this section (as well as in section V) the following pertains: 1) the value of the phase at the top is $+\pi$ whereas at the bottom the phase value is $-4 \pi$; and 2) the phase curve (the dashed curve other than the dashed Gaussian curve) is relative to the maximum value of the normalized amplitude of the E-Field distribution where at this point the phase is defined as zero radians. A positive phase value indicates a leading value of the phase of the wave front and a negative phase value indicates a lag in phase with respect to the phase of the maximum value of the normalized amplitude.

V) This graph applies to mirror $\mathrm{M}_{2}$. Refer to section U's explanation.

W) This graph applies to mirror $\mathrm{M}_{2}$. The explanation of this section is similar to section $\mathrm{T}$.

$\mathrm{X})$ This is an illustration of mirror $\mathrm{M}_{2}$.

$\mathrm{Y})$ This graph is mirror $\mathrm{M}_{1}$ 's convergence of decay per round-trip vs. roundtrip. The decay/RT, the vertical axis, is a linear scale. The graph is a measure of the change in $\mathrm{M}_{1}$ 's area under the E-Field distribution relative to the last area's value. The horizontal line in the graph show the zero level. 
When the decay/RT is plotted at the zero level, no change in the area under the normalized amplitude curve has occurred in two consecutive round-trips at mirror $\mathrm{M}_{1}$. At the bottom of the section is $\mathrm{M}_{1}$ 's power loss relative to the energy reflected from mirror $\mathrm{M}_{2}$.

Z) This is a graph of the percent power loss P.L. per pass per mirror vs. pass or transit. The P.L./Pass/Mirror, the vertical axis, is a log to the base 10 scale. For example: A value of two, the maximum attainable value, represents a $100 \%$ power loss and a value of zero represents a $1 \%$ power loss per pass. Three curves are plotted, one is a zigzag and the others are envelopes. One of the envelope curves begins at the first transit, this is the power loss due to mirror $\mathrm{M}_{1}$. The other envelope curve begins at the second transit and is $\mathrm{M}_{2}$ 's power loss curve. The zigzag curve just connects the two envelope curves and indicates sequential power loss of the system of two mirrors. At the bottom of the section is the either $\mathbf{M}_{1}$ 's or $\mathbf{M}_{2}$ 's power loss relative to the energy reflected from the other mirror depending upon which transit the beam is on.

$\lambda$ ) This is $\mathbf{M}_{2}$ 's graph of the decay/RT vs. round-trip. The explanation of this section is similar to section $\mathrm{Y}$.

We begin the computer simulations by investigating the effect of mirror radius with respect to the theoretical waist at the mirrors of a symmetric confocal resonator. The theory described in Chapter II does not take the mirror radius into account.

First, let us determine the power in a transverse infinite plane that is carried by the fundamental mode of a Gaussian beam. Then, find the power contained in a transverse circular plane of finite radius equalling the mirror radius.

The power $\mathrm{P}$ is defined as

$$
P=\int I(x, y, z) d a,
$$


where $I(x, y, z)=|E(x, y, z)|^{2}=$ the optical intensity and da is an incremental area. This can be rewritten as

$$
P=\int I(\rho, z) d a=\int I_{0}\left[\frac{w_{0}}{w(z)}\right]^{2} \exp \left[-\frac{2 \rho^{2}}{w^{2}(z)}\right] d a,
$$

where $I_{0}=\left|E_{0}\right|^{2}$ and $\rho^{2}=x^{2}+y^{2}$.

The power carried by the beam of infinite extent in the transverse direction is

$$
\mathrm{P}_{\rho=\infty}=\mathrm{I}_{0}\left[\frac{\mathrm{w}_{0}}{\mathrm{w}(\mathrm{z})}\right]^{2} \int_{0}^{\infty} \exp \left[-\frac{2 \rho^{2}}{\mathrm{w}^{2}(\mathrm{z})}\right] \rho d \rho \int_{0}^{2 \pi} \mathrm{d} \varphi
$$

which reduces to

$$
\mathrm{P}_{\rho=\infty}=1 / 2 \mathrm{I}_{0}\left(\pi \mathrm{w}_{0}{ }^{2}\right) .
$$

Thus the power is $1 / 2$ the peak intensity times the beam area.

Now we will determine the power contained within the finite circular area of radius equal to the mirror radius $r$. We have

$$
P_{\rho=r}=I_{0}\left[\frac{w_{0}}{w(z)}\right]^{2} \int_{0}^{r} \exp \left[-\frac{2 \rho^{2}}{w^{2}(z)}\right] \rho d \rho \int_{0}^{2 \pi} d \varphi,
$$

which reduces to

$$
P_{\rho=r}=1 / 2 I_{0}\left(\pi w_{0}^{2}\right)\left[1-\exp \left[-\frac{2 r^{2}}{w^{2}(z)}\right]\right]
$$

The percent of the total power $\mathrm{P}_{\mathrm{T}}$ carried within a circle of radius $r$ is given by

$$
\% \mathrm{P}_{\mathrm{T}}=\frac{\mathrm{P}_{\rho=\mathrm{r}}}{\mathrm{P}_{\rho=\infty}} \times 100 \%=\left[1-\exp \left[-\frac{2 \mathrm{r}^{2}}{\mathrm{w}^{2}(\mathrm{z})}\right]\right] \times 100 \% .
$$

For example, the power contained inside a circle of radius $\mathrm{r}=\mathrm{w}(\mathrm{z})$ is about $86 \%$ of the total power. In other words, about $16 \%$ of the total power spills over the mirror's edge and is lost in the open unwalled resonator.

The beam radius at the mirrors of a Symmetric Confocal Resonator is 
$w=(\lambda d / \pi)^{1 / 2}$. Since the Fresnel number $N$ equals $r^{2} / \lambda d$, we can say $N=r^{2} / \pi w^{2}$. The loss in the Symmetric Confocal Resonator is governed by this Fresnel number N; a higher value of $\mathrm{N}$ means a smaller loss.

Figure 23 shows a plot of the difference between the theoretical and simulated power that spills over the mirror's edge in a Symmetric Confocal Resonator $(d / R=1)$ as well as a Symmetric Resonator where $d / R=1.5$. The percent theoretical power loss is $\% \mathrm{P}_{\mathrm{T}}$ as is defined above (the value of the mirror radius $\mathrm{r}$ is found from a given $\mathrm{N}$ value where $\mathrm{r}=(\mathrm{N} \lambda \mathrm{d})^{1 / 2}$ and the value of the waist at the mirrors $\mathrm{w}_{1,2}$ in a Symmetric Resonator is given by $\left.\mathrm{w}_{1,2}=(\lambda \mathrm{d} / 2 \pi \mathrm{n})^{1 / 2}\left[2 \mathrm{R}^{2}(\mathrm{~d}(\mathrm{R}-\mathrm{d} / 2))\right]^{1 / 4}\right)$. The simulated power loss is obtained

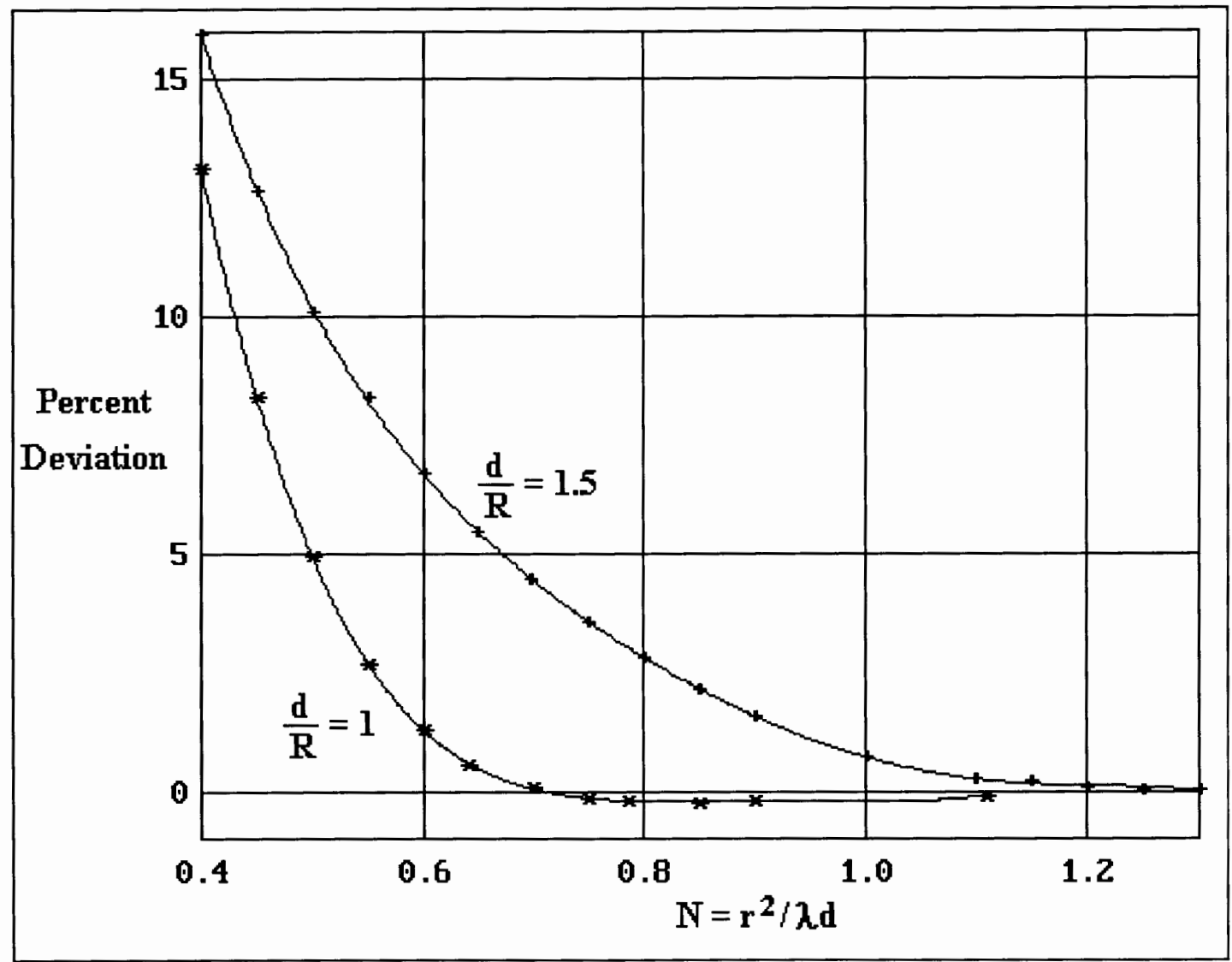

Figure 23. The difference between the theoretical and simulated values of loss per transit vs. the Fresnel number of a Symmetric Confocal Resonator $(d / R=1)$ and another Symmetric Resonator where $d / R=1.5$. 
from a computer run for a particular value of $\mathrm{N}$. The Percent Deviation axis in the figure is defined as the difference between the simulated and theoretical power losses. The figure indicates that as $\mathrm{N}$ increases the simulated loss per transit approaches the theoretical value. This is because the theory assumes an effective mirror radius equal to infinity.

A trend-plot of four computer runs of a Symmetric Confocal Resonator where the mirror radius is varied from $r=w$ to $r=2 w$ is shown in Figures 24 and 25. Notice the quick convergence of the E-Field to the theoretical dotted Gaussian profile in the number of round-trips. In the bottom of Figure 25 convergence to this Gaussian profile is not even close. The higher transverse modes are still in competition for oscillation even though 300 round-trips have transpired. In this instance 500 to 600 round-trips may be required such that the steady state profile is reasonably close to the theoretical Gaussian profile. Another trend-plot of the percent completion of a Symmetric Resonator where $\mathrm{d} / \mathrm{R}=1.5$ is shown in Figures 26 and 27 . The percent completion is varied from $25 \%$ to $100 \%$. Actually the steady state has not quite been reached as indicated in the bottom of Figure 27 by the dev_amp value of $-1.85 e-01 \%$ as well as the decay graphs not quite reaching zero percent. This is close enough though for comparison purposes to call this $100 \%$ complete after 200 round-trips where 250 round-trips may be required for full convergence. The important issue of this trend plot is the profile of the beam that is shown in the middle graphs (sections $\mathrm{U}$ and $\mathrm{V}$ ) of the four computer simulations.

A comparison between four Symmetric Confocal Resonators is shown in Figures 28 and 29 where the mirror type is varied. Figure 28 shows there is no difference in the loss per transit between Spherical and Fresnel Mirror types. Figure 29 shows that as the number of tiers per zone of the Tiered Fresnel Mirror is increased the loss per transit is decreased. Remember, as the limit of the number of tiers per zone approaches infinity the Tiered Fresnel Mirror becomes the Fresnel Mirror. 


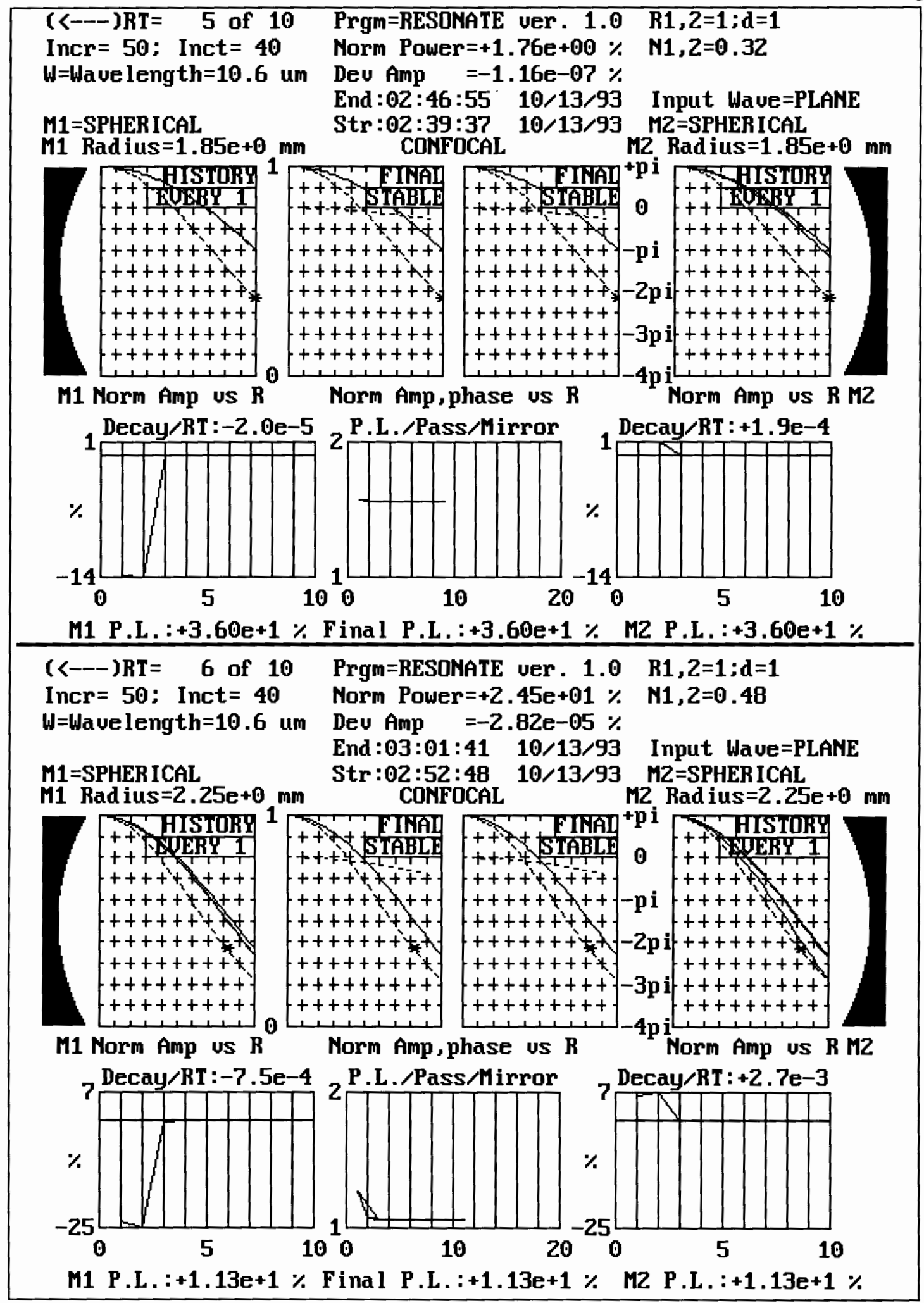

Figure 24. The mirror radius varies in a Symmetric Confocal Resonator in Figures 24 and 25. The top has $r=w$ and the bottom has $r=1.2 \mathrm{w}$. 


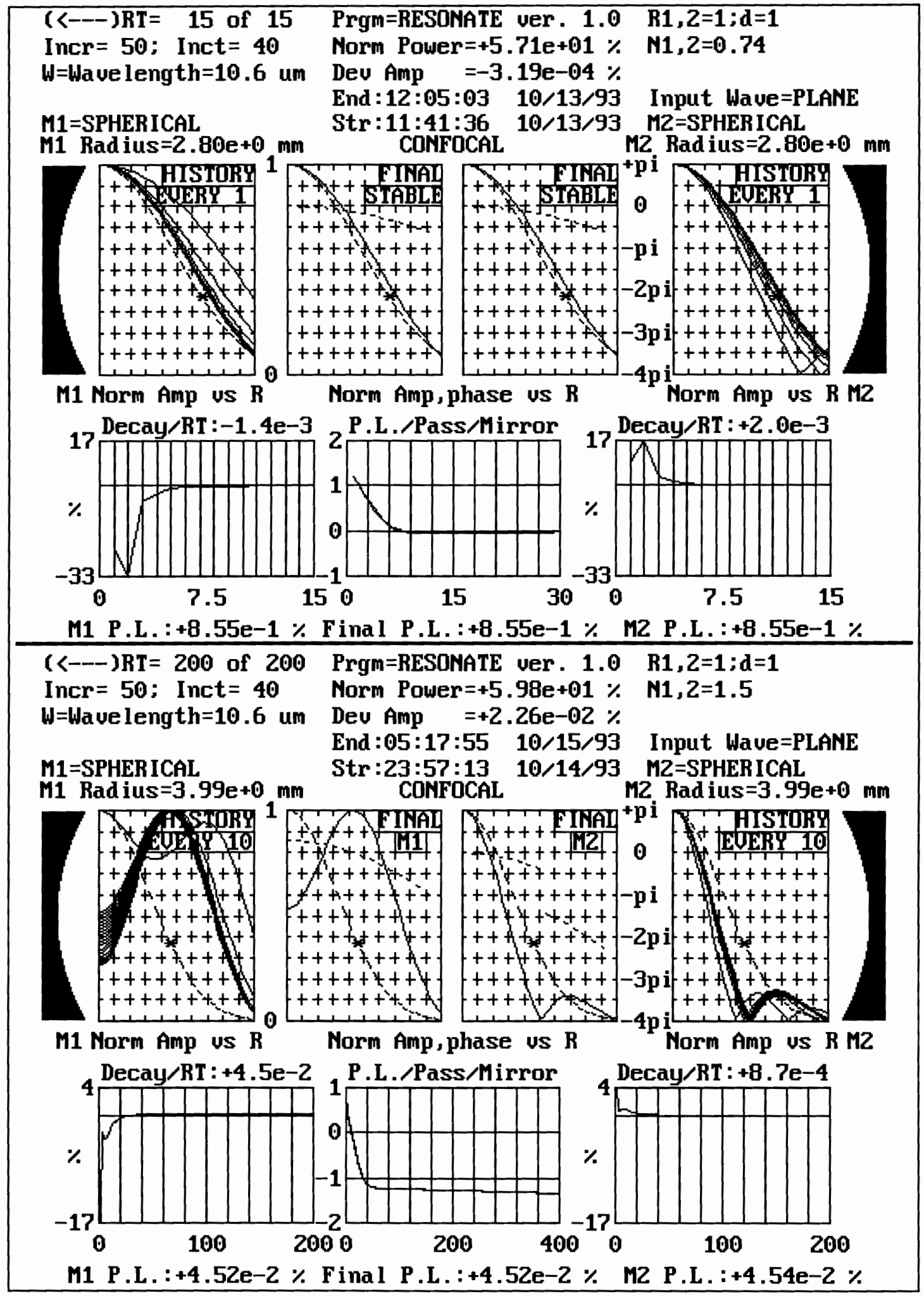

Figure 25. The mirror radius varies in a Symmetric Confocal Resonator in Figures 24 and 25. The top has $r=1.5 \mathrm{w}$ and the bottom has $r=2 \mathrm{w}$. 


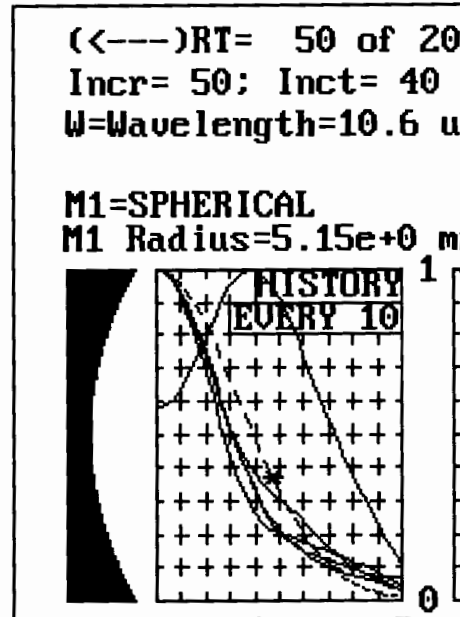

M1 Norm Amp us $R$
Prgm=RESOMATE ver .1 .0

Norm Power $=+4.83 e+01 \%$

Dev Amp $=-1.19 \mathrm{e}+00 \%$

End : 15:04:34 10/12/93

Str:13:44:45 10/12/93
$R 1,2=1: d=1.5$

$N 1,2=1.66$

Input Wave $=$ PLANE

$M 2=$ SPHERICAL

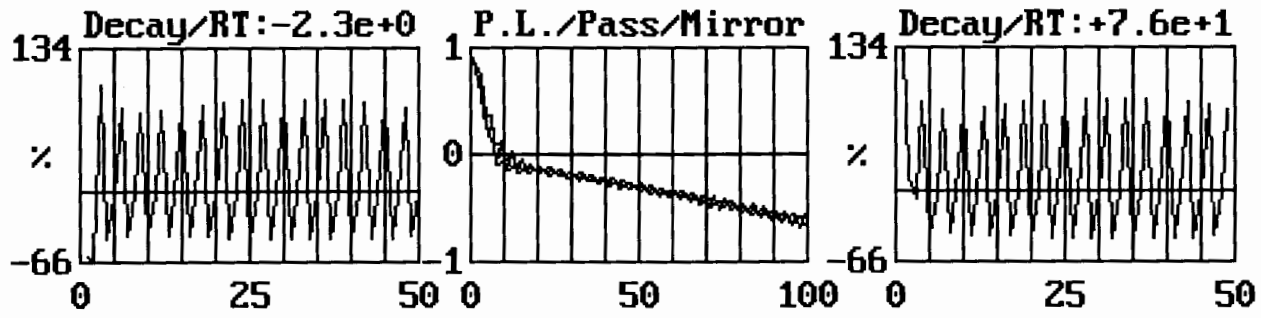

M1 P.L. : +2.29e-1\% Final P.L. : $+2.29 \mathrm{e}-1 \%$ MZ P.L. $:+2.40 \mathrm{e}-1 \%$

$(<--) R T=100$ of 200 Prgm=RESONATE ver. $1.0 \quad \mathrm{R} 1, z=1 ; \mathrm{d}=1.5$

Incr $=50 ;$ Inct $=40$

$W=$ Wave length $=10.6 \mathrm{um}$

Norm Power $=+4.31 \mathrm{e}+01 \%$

$N 1, Z=1.66$

Dev Amp $=-1.14 \mathrm{e}+01 \%$

End:13:41:13 10/12/93 Input Wave=PLANE

M1=SPHERICAL

Str:11:01:32 10/12/93 M2=SPHERICAL

M1 Radius $=5.15 \mathrm{e}+0 \mathrm{~mm}$

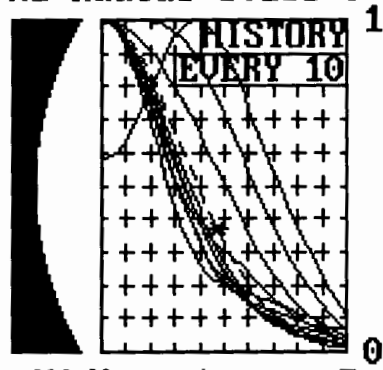

M1 Norm Amp us $R$

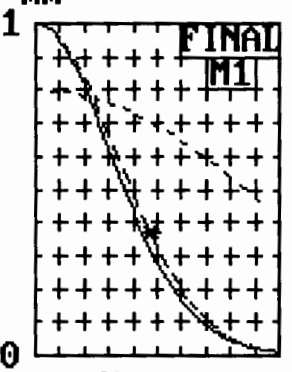

Norm Amp, phase us $R$

M2 Radius $=5.15 \mathrm{e}+0 \mathrm{~mm}$

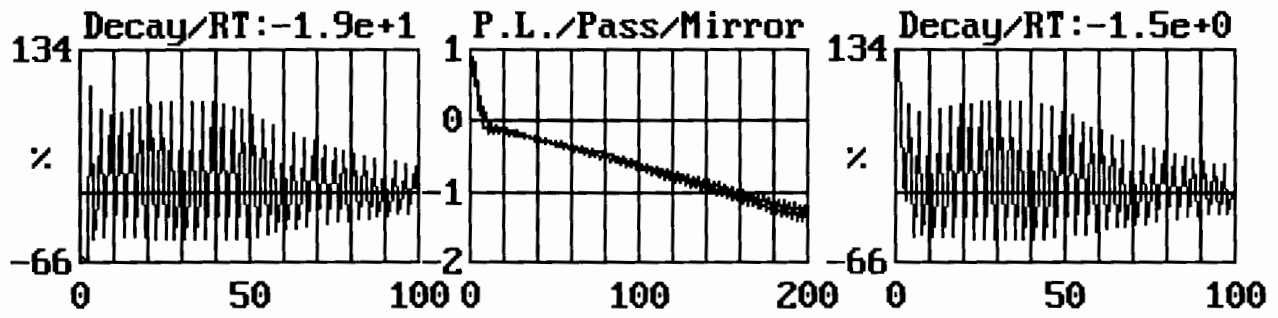

M1 P.L.:+3.57e-2 \% Final P.L.: +3.57e-2\% M2 P.L. :+3.75e-2\%

Figure 26. A 25\% (top) and a 50\% (bottom) completed run of a Symmetric Resonator $(\mathrm{d} / \mathrm{R}=1.5)$. Compare with Figure 27. 
$(<--) R T=150$ of 200

Incr $=50 ;$ Inct $=40$

$W=$ Wave length $=10.6 \mathrm{um}$

$M 1=$ SPHER ICAL

M1 Radius $=5.15 e+0 \mathrm{~mm}$
Prgm=RESOMATE ver $.1 .0 \quad R 1,2=1 ; d=1.5$

Morm Power $=+4.19 \mathrm{e}+01 \% N 1,2=1.66$

Deu Amp $=+4.12 \mathrm{e}+00 \%$

End :08:54:18 10/12/93 Input Wave=PLANE

Str:04:54:49 10/12/93 M2=SPHERICAL M2 Radius $=5.15 \mathrm{e}+0 \mathrm{~mm}$

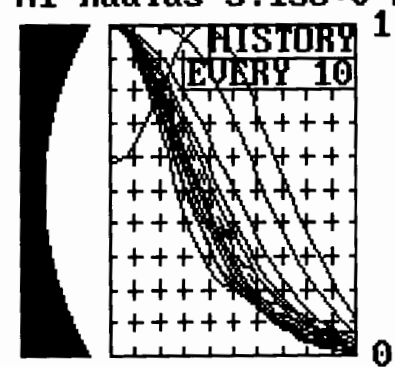

M1 Morm Amp us $R$

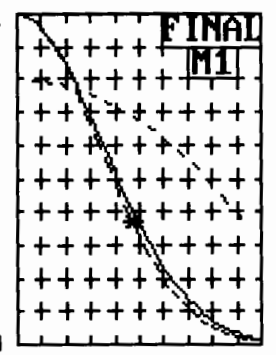

Norm Amp, phase us $\mathbf{R}$

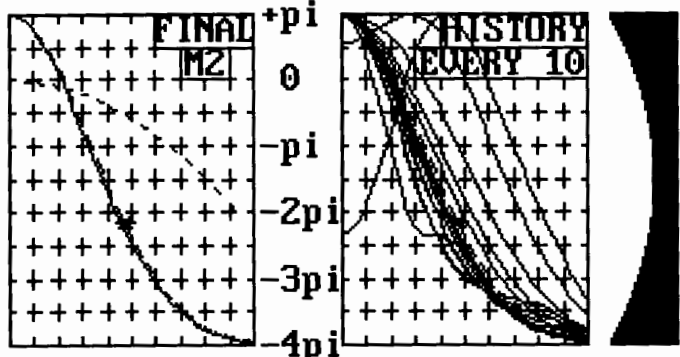

Norm Amp us R M2

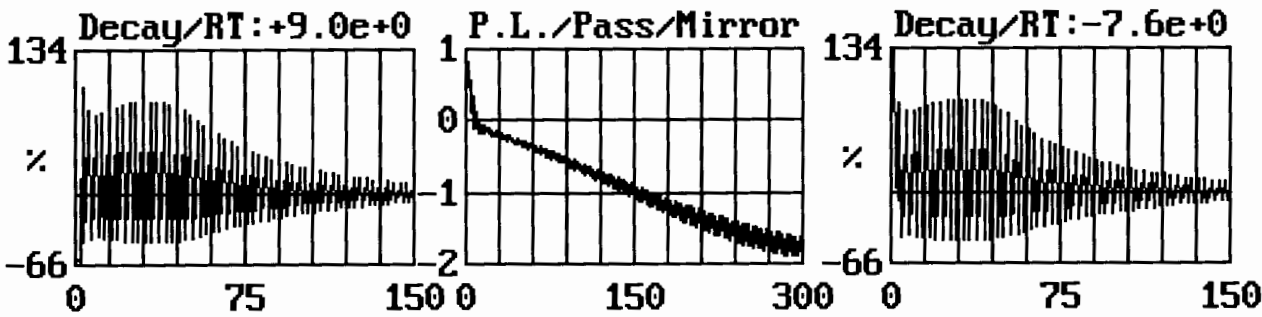

M1 P.L. : +2.21e-2 \% Final P.L. $:+2.21 \mathrm{e}-2 \%$ M2 P.L. $:+2.26 \mathrm{e}-2 \%$

$(<--) R T=200$ of 200 Prgm=RESONATE ver . 1.0 R1, $2=1 ; d=1.5$

Incr $=50 ;$ Inct $=40 \quad$ Norm Power $=+4.14 \mathrm{e}+01 \% \quad \mathrm{~N} 1, Z=1.66$

$W=$ Wave length $=10.6 \mathrm{um}$

Dev Amp $=-1.85 \mathrm{e}-01 \%$

End:06:48:53 10/01/93 Input Wave=PLANE

M1=SPHERICAL

Str:01:22:16 10/01/93 M2=SPHERICAL

M1 Radius $=5.15 \mathrm{e}+0 \mathrm{~mm}$

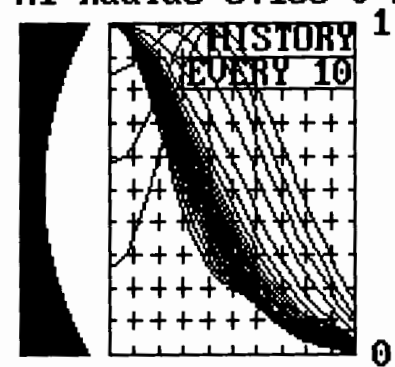

M1 Norm Amp us $\mathbf{R}$

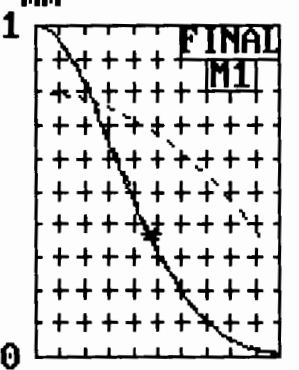

Norm Amp, phase us $R$

MZ Radius $=5.15 \mathrm{e}+0 \mathrm{~mm}$

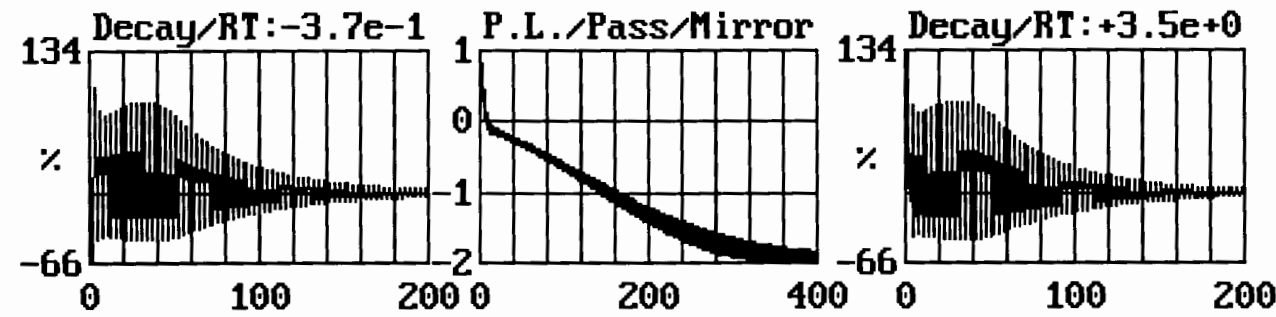

M1 P.L.: +1.16e-2 \% Final P.L.:+1.16e-2\% M2 P.L.:+1.16e-2\%

Figure 27. A $75 \%$ (top) and a 100\% (bottom) completed run of a Symmetric Resonator $(\mathrm{d} / \mathrm{R}=1.5)$. Compare with Figure 26. 


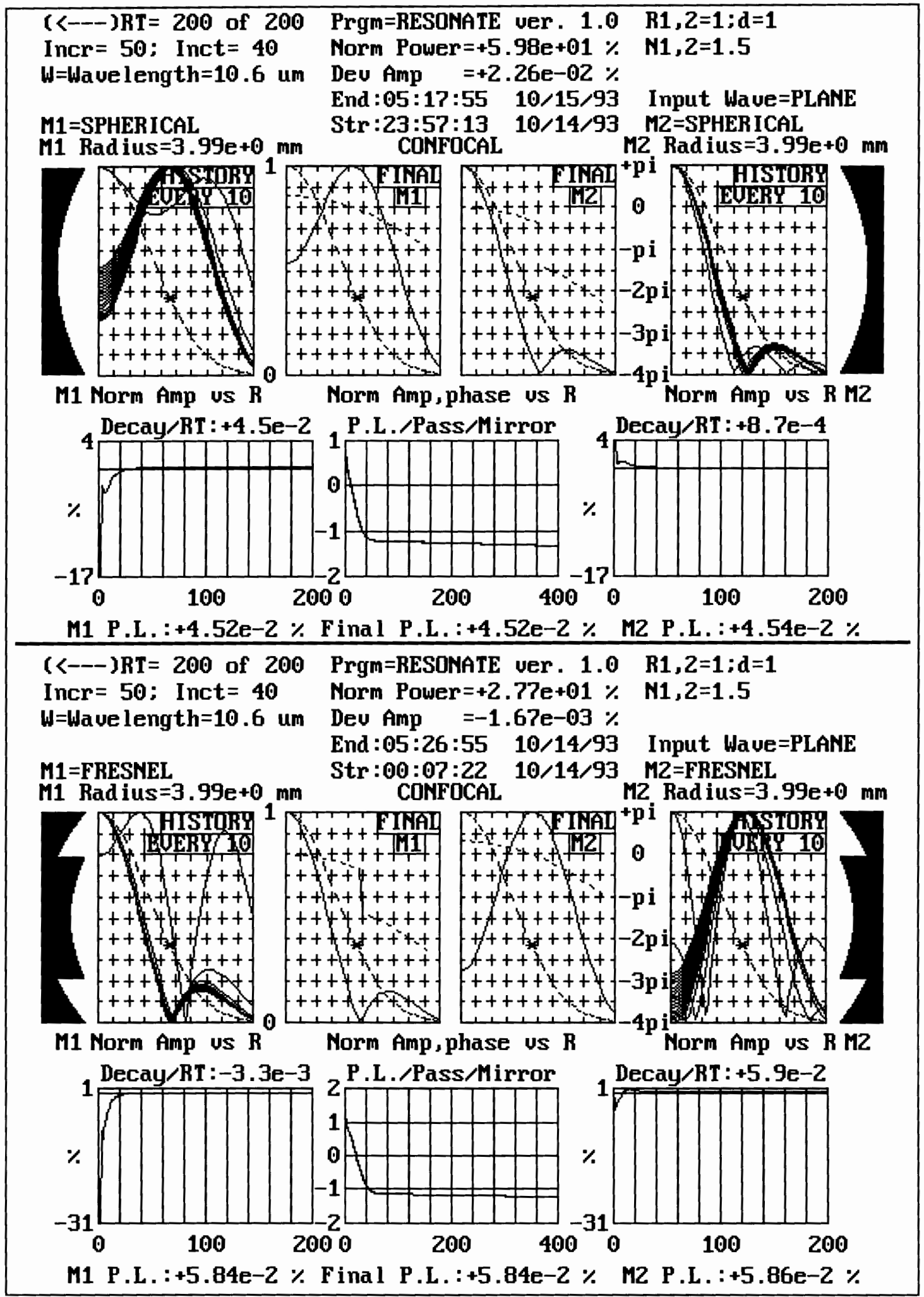

Figure 28. A comparison of mirror types of a Symmetric Confocal Resonator. Shown is a Spherical type (top) and a Fresnel type (bottom). 
$(\angle--) R T=200$ of 200

Incr $=50:$ Inct $=40$

$W=$ Wave length $=10.6 \mathrm{um}$

Depth1 $2=W /(30,30)$

$M 1=T$ IERED (15)

M1 Radius $=3.99 \mathrm{e}+0 \mathrm{~mm}$
Prgm=RESONATE ver, 1.0

Morm Power $=+5.41 \mathrm{e}-02 \%$

Deu Amp $=+3.55 e-02 \%$

End : $22: 21: 12 \quad 10 / 14 / 93$

Str:17:01:02 10/14/93
$R 1,2=1 ; d=1$

$M 1,2=1.5$

tiers1,2=23,23

Input Wave=PLANE

MZ= T IERED (15)

12 Radius $=3.99 \mathrm{e}+0 \mathrm{~mm}$

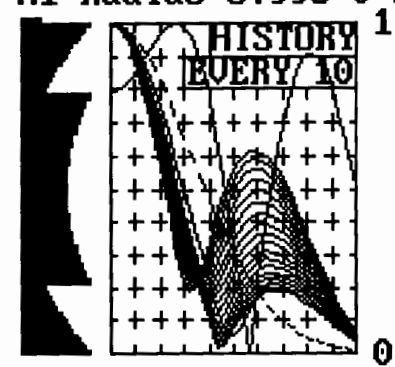

M1 Norm Amp us $R$

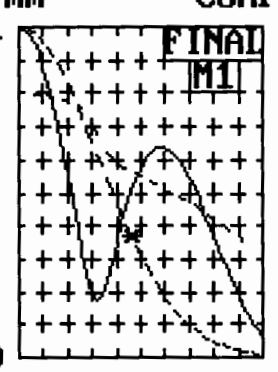

Norm Amp, phase us $\mathbf{R}$

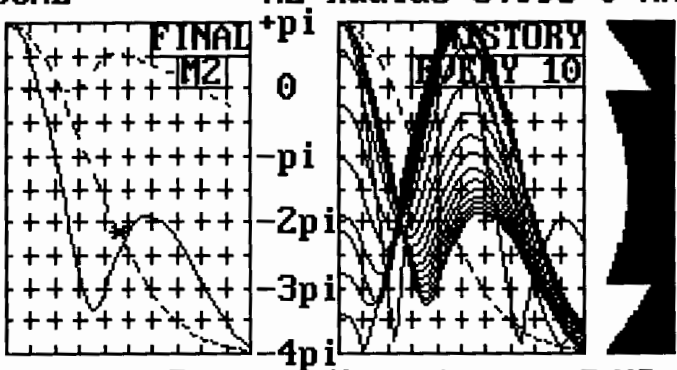

Norm Amp us $\mathrm{R}$ M2

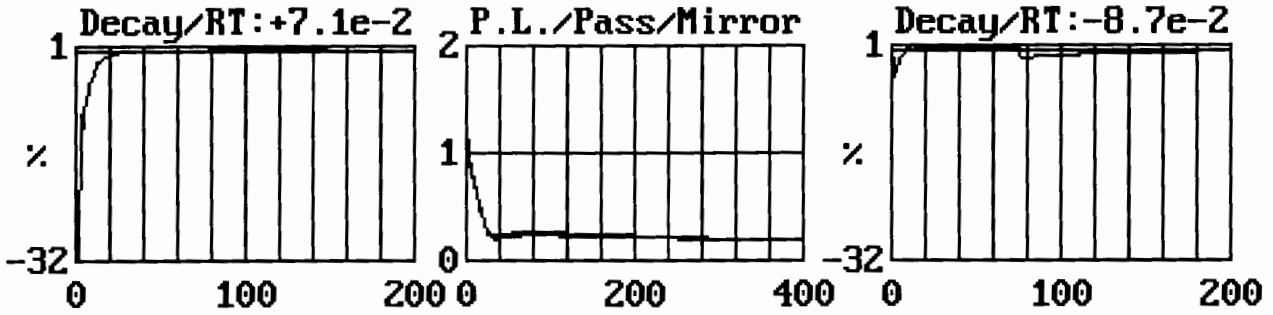

M1 P.L.: $+1.55 \mathrm{e}+0 \%$ Final P.L. $:+1.55 \mathrm{e}+0 \%$ M2 P.L. $:+1.52 \mathrm{e}+0 \%$

$(<--) R T=200$ of 200 Prgm=RESONATE ver. $1.0 \quad R 1,2=1 ; d=1$

Incr $=50 ;$ Inct $=40$

$W=$ Wave length $=10.6 \mathrm{um}$

Depth1, $Z=W /(10,10)$

M1=T IERED (5)

Norm Power $=+2.66 \mathrm{e}-20 \% \mathrm{N1}, 2=1.5$

Dev Amp $=+3.06 \mathrm{e}-02 \%$ tiers $1,2=8,8$

End:16:01:53 10/14/93 Input Wave=PLANE

Str: 10:42:27 10/14/93 MZ=T IERED(5)

M1 Radius $=3.99 \mathrm{e}+0 \mathrm{~mm}$ CONFOCAL

M2 Radius $=3.99 \mathrm{e}+0 \mathrm{~mm}$

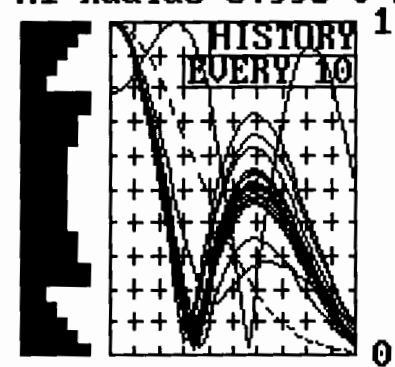

M1 Norm Amp us $R$

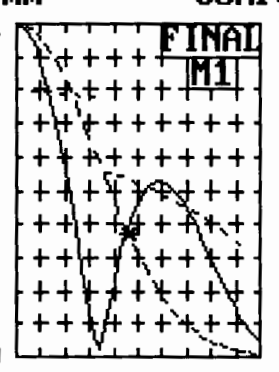

Norm Amp, phase us $\mathbf{R}$

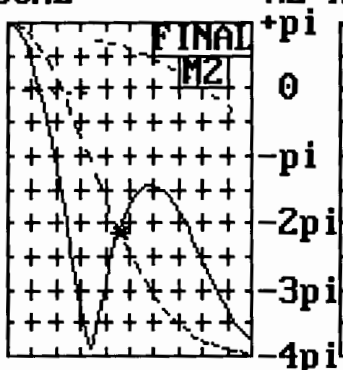

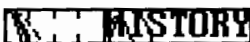

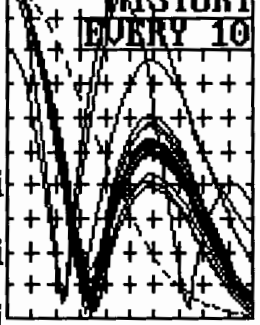

Norm Amp us $\mathrm{R}$ M2

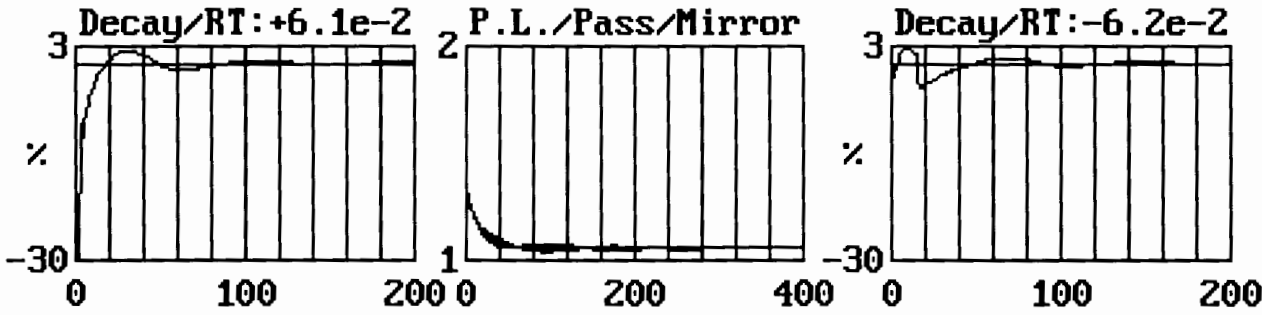

M1 P.L.: $+1.13 e+1 \%$ Final P.L.: $+1.13 e+1 \%$ MZ P.L. $:+1.14 \mathrm{e}+1 \%$

Figure 29. A Tiered(15) Fresnel (top) and a Tiered(5) Fresnel (bottom) Mirror types of a Symmetric Confocal Resonator. See Figure 28. 
Another comparison between these four mirror types is shown in Figures 30 and 31 where a symmetric resonator of $\mathrm{d} / \mathrm{R}=1.5$ is used. Notice that for a given Fresnel number both the waist at the mirrors and the loss per transit are larger than for the symmetric confocal resonator. The diffraction loss can be readily seen from Figure 32.

Figure 32 is a paramount figure because it describes the feasibility of the tiered mirror's performance. It is shown that a Fresnel Mirror having more tiers per zone will have a better performance. This is because, when going from a more planar structure to that of a more curved one, better confinement of the beam occurs. Note also that the Fresnel Mirror and the Spherical Mirror has virtually identical loss profiles. This is due to both of the mirror's surfaces are constant phase surfaces. Also note that the leveling off of the power loss for the Tiered Fresnel Mirrors as the Fresnel Number increases is a possible artifact of the the resolution of the integration performed over the mirror surfaces. It is conjectured that as the number of increments is increased a more of a gradual leveling would occur.

A designer of a laser resonator system has to know the diffraction loss of the mirrors (found from Figure 32) as well as the gain of the laser medium. A high loss mirror teamed with a low gain laser medium is not a good match but rather just the opposite. For example, a tiered(5) Fresnel mirror (a tiered mirror with five zones per tier) in a $\mathrm{d} / \mathrm{R}=1.5$ symmetric resonator has a diffraction loss per transit of approximately $14 \%$ for a Fresnel number of one (see bottom of Figure 32). When teamed with a HeNe lasing medium of approximately $2 \%$ gain per transit it will not operate because amplification of the light is impossible due to a higher loss than gain. However, when a tiered(15) Fresnel mirror in a symmetric resonator of $\mathrm{d} / \mathrm{R}=1.5$ is teamed with a $\mathrm{CO}_{2}$ laser medium, operation is now possible. This is because the diffraction loss per transit is approximately $1.5 \%$ while the $\mathrm{CO}_{2}$ laser medium is approximately $10 \%$ gain per transit. 


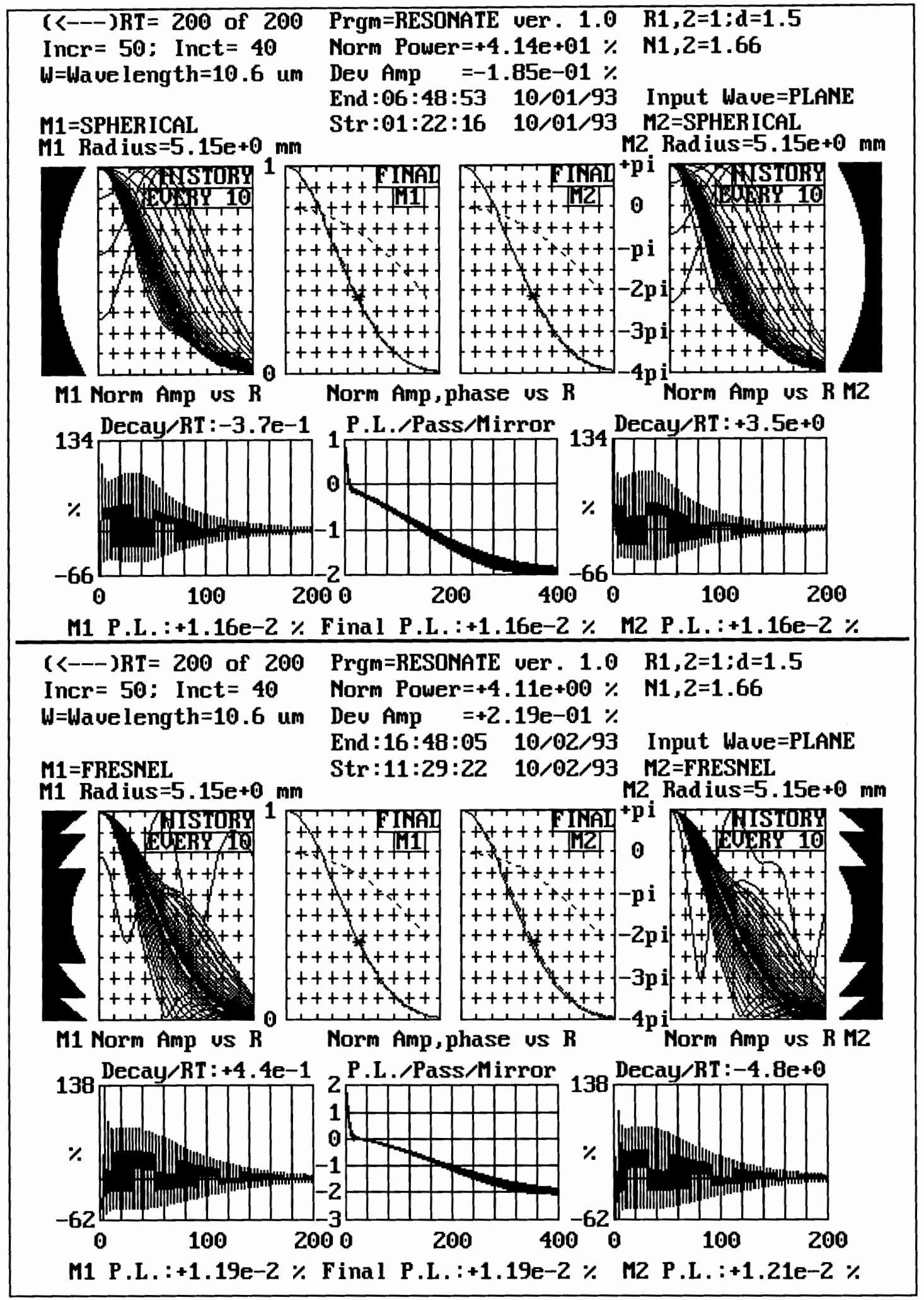

Figure 30. A comparison of mirror types of a $\mathrm{d} / \mathrm{R}=1.5$ Symmetric

Resonator. Shown is a Spherical type (top) and a Fresnel type (bottom). 


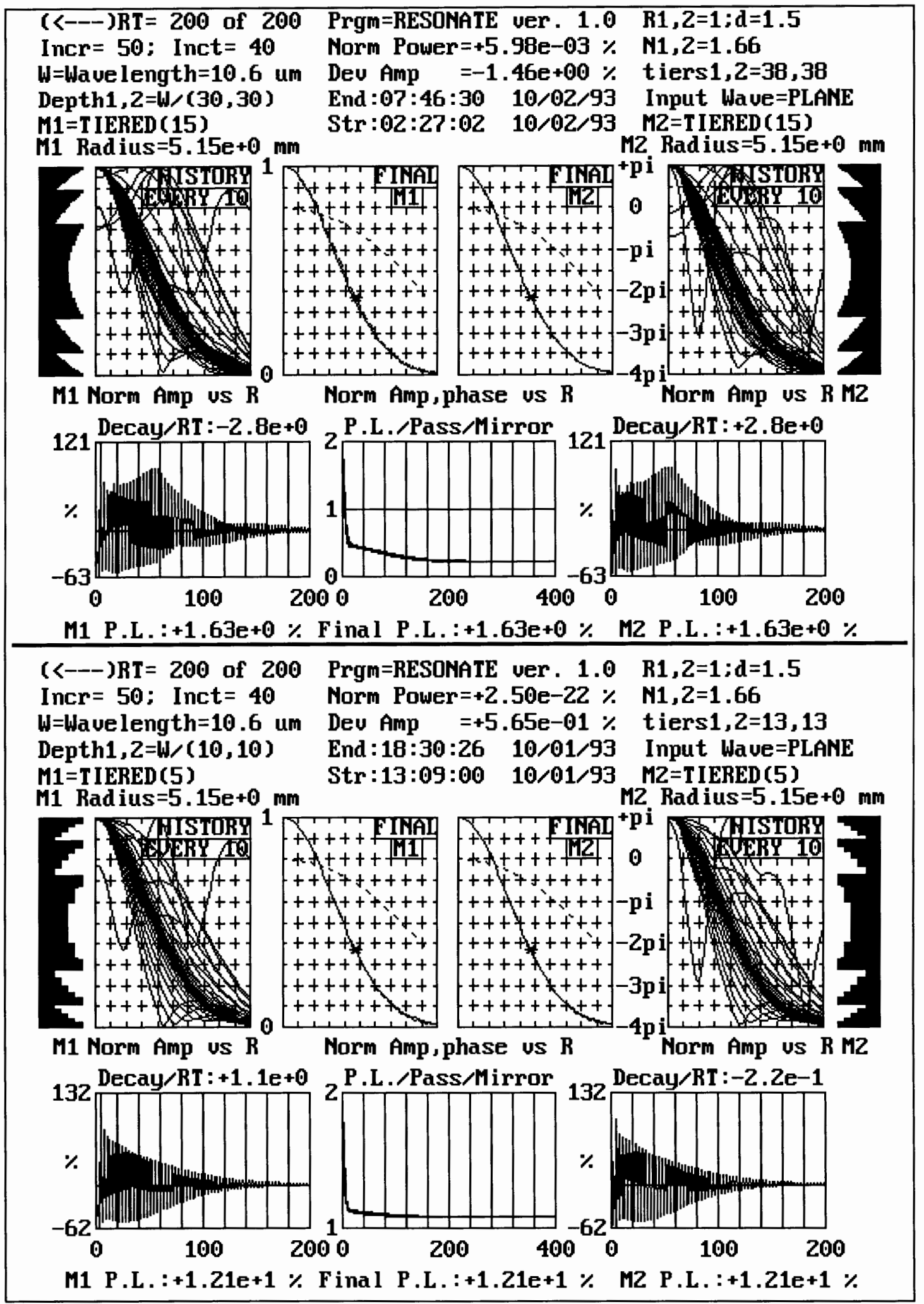

Figure 31. A Tiered(15) Fresnel (top) and a Tiered(5) Fresnel (bottom) Mirror types of a d/R=1.5 Symmetric Resonator. See Figure 30. 


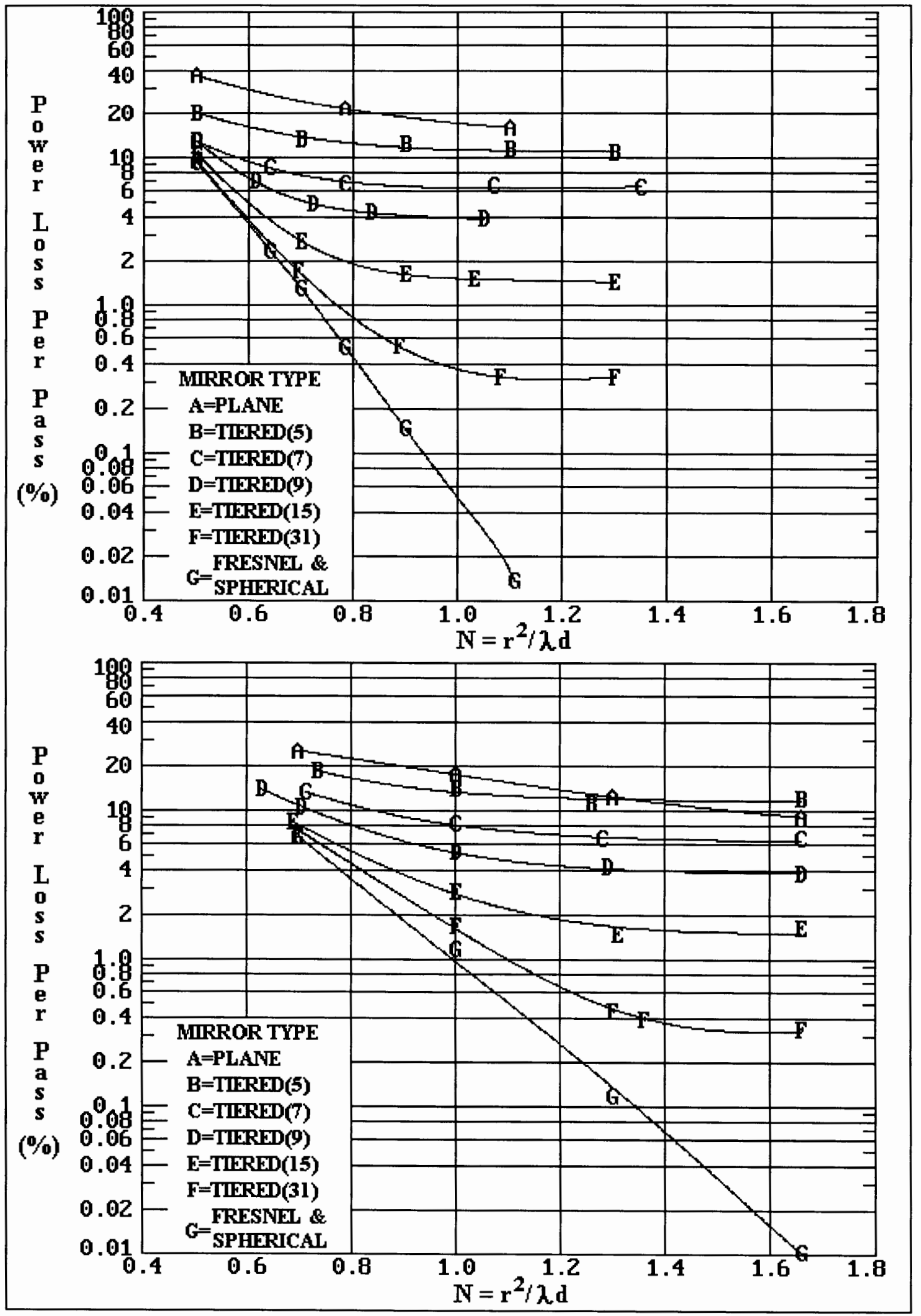

Figure 32. Mirror performances of a $d / R=1$ and a $d / R=1.5$ Symmetric Resonator. 


\section{CHAPTER V}

\section{INTEGRATED CIRCUITS PROCESSING}

Tiered Fresnel Mirrors can be made efficiently in quantity by using segments of the Integrated Circuits Process. These segments consist of : 1) Photolithography; 2) Plasma Etching and 3) Metal Deposition. Also Low Pressure Chemical Vapor Deposition, LPCVD, oxide and LPCVD nitride may be used.

Two types of Tiered Fresnel Mirrors can be manufactured. One type is a simpler reflective mirror whereas the other type is a more involved transmittant mirror. The reflective type may be best used with a plane mirror. This configuration is the HalfSymmetric Resonator. The reflective mirror is made by sequential patterning and etching to form the tiers. Then a metal deposition is performed to form the fully reflective surface of the mirror. This mirror configuration is shown in Figure 33.

The other transmittant mirror type can be made by multiple depositions of LPCVD oxide and LPCVD nitride, patterning, etching and a metal deposition for the partially transmittant mirror surface. A cross-sectional view is shown in Figure 34. A zoom of this mirror is shown in Figure 35. The wavefront of the beam is concave curved when arriving at the mirror surface. What transmits through the metallic film exits through the mirror with a wavefront that is parallel to the back side of the mirror surface. This can be seen by observing that the sinusoidal curves, representing the E-Field distribution of the beam, are all in the same phase at the back side of the mirror. The amplitude of the sinusoidal curves is the same to illustrate the phase retardation when travelling through the mirror whereas the amplitude is exponentially diminished in the radial dimension as shown in Figure 34. 


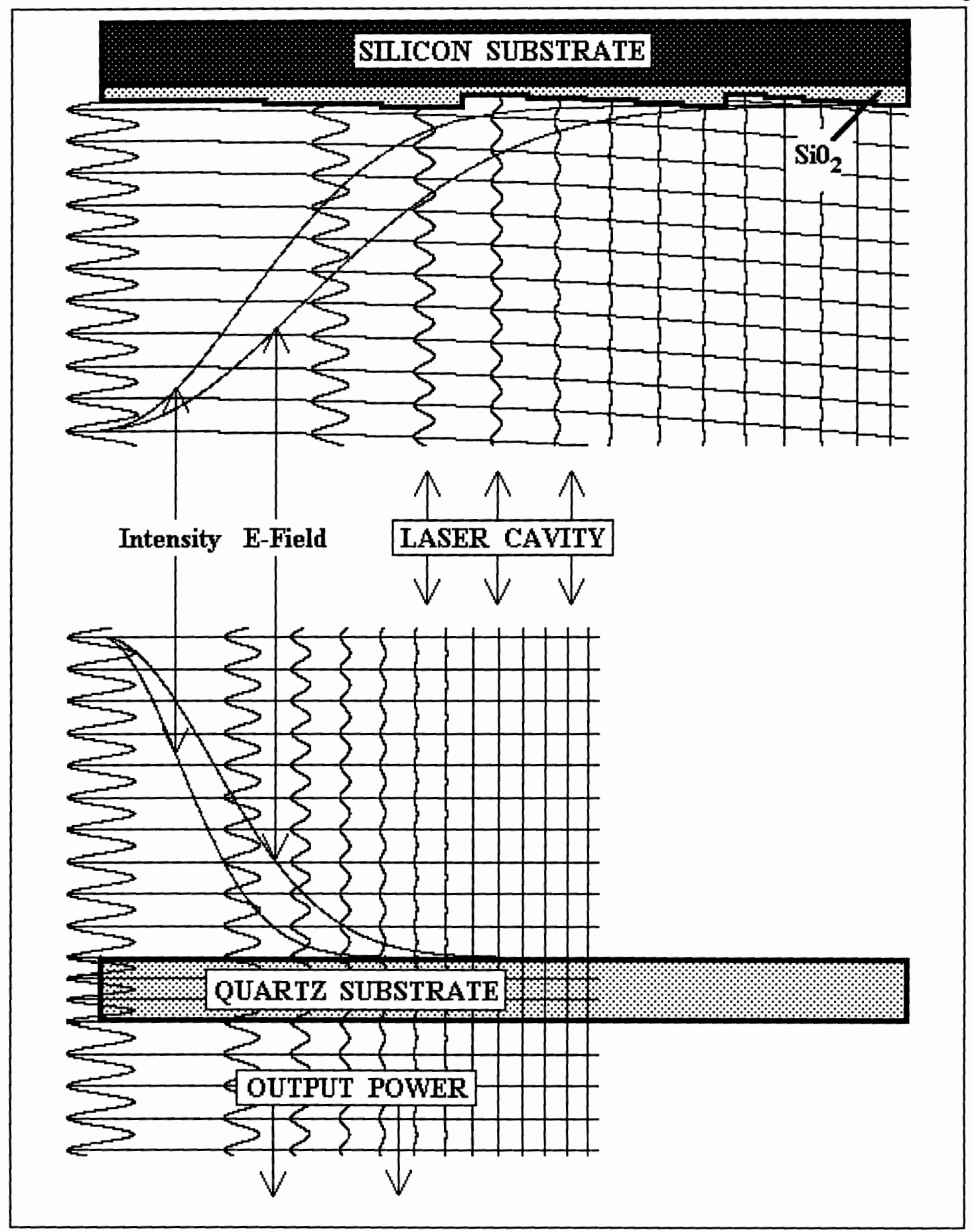

Figure 33. A radial cross-sectional view of a fully reflective Tiered(5) Fresnel Mirror with a partially transmitting Plane Mirror. Note: This is a Half-Symmetric Resonator. 


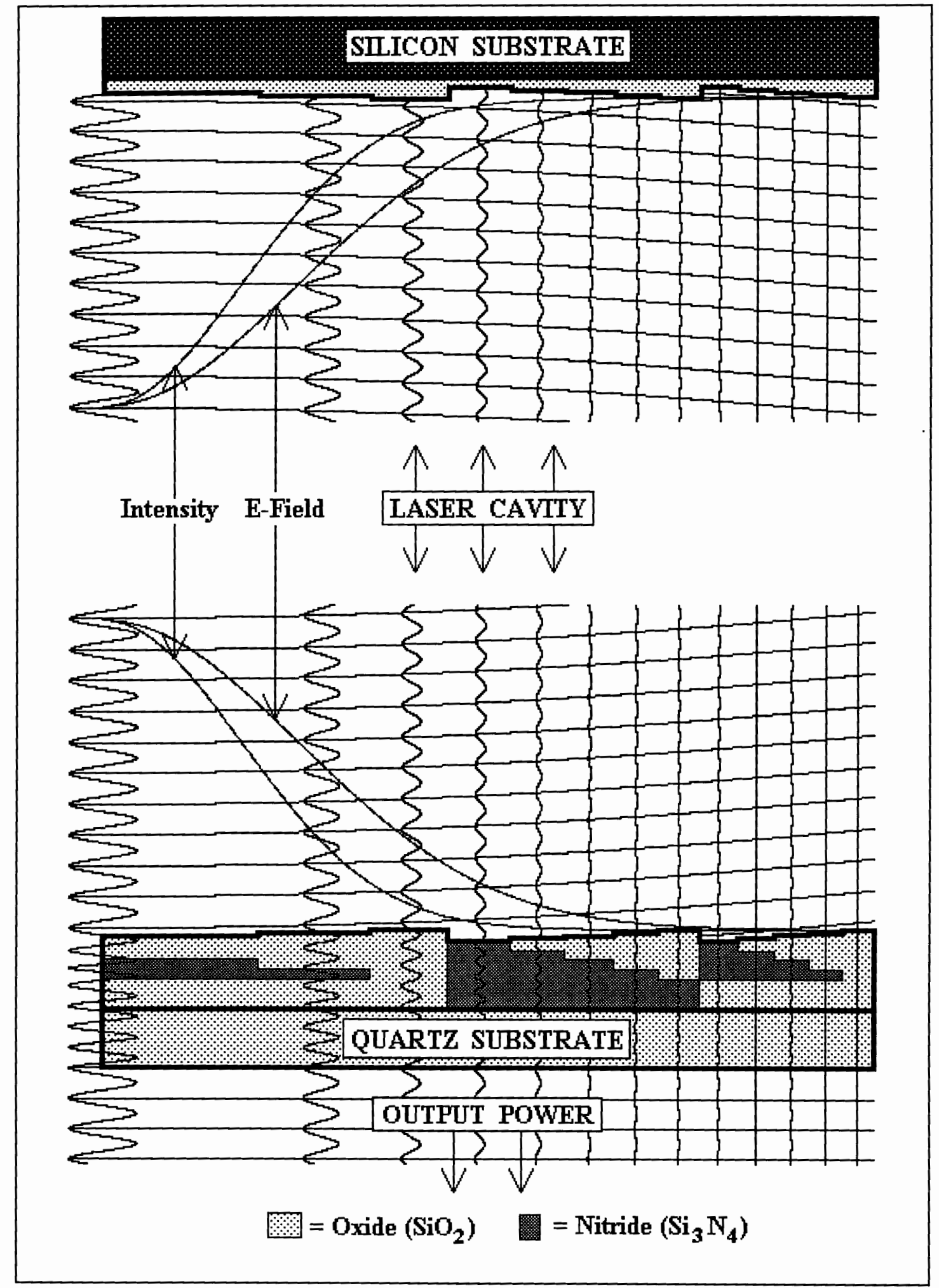

Figure 34. A radial cross-sectional view of a fully reflective and a partially transmitting Tiered(5) Fresnel Mirrors. 


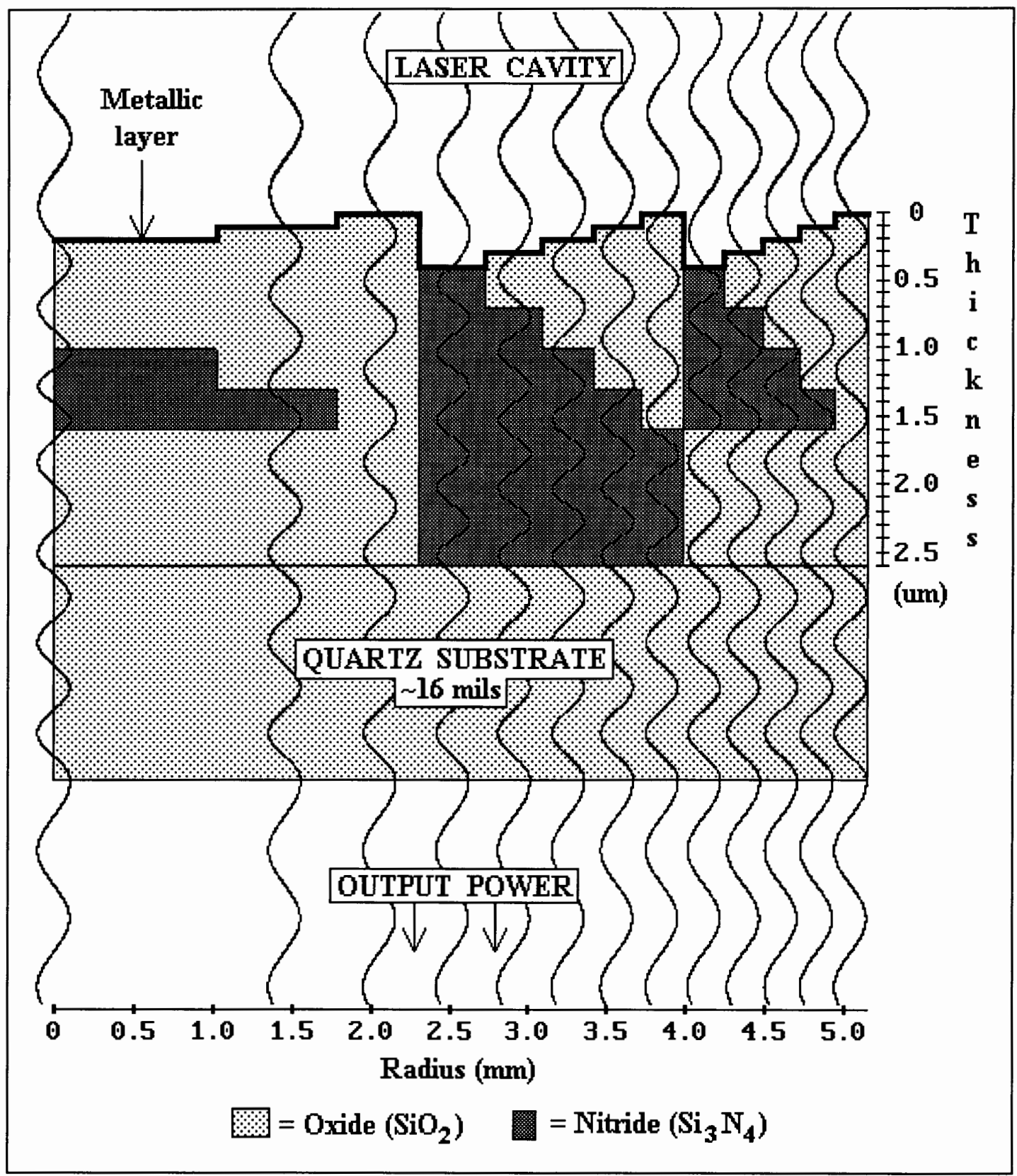

Figure 35. A zoom-in of the bottom mirror in Figure 34. The particular arrangement and thicknesses of the Oxide and Nitride film stacks convert a curved wavefront into a planar wavefront.

Figure 37 shows a sequence of cross-sections for the formation of the tiers from start to finish of a "five tiers per zone " reflective type Tiered Fresnel Mirror, i.e. a reflective type Tiered(5) Fresnel Mirror. A photomask is used to define the photoresist 


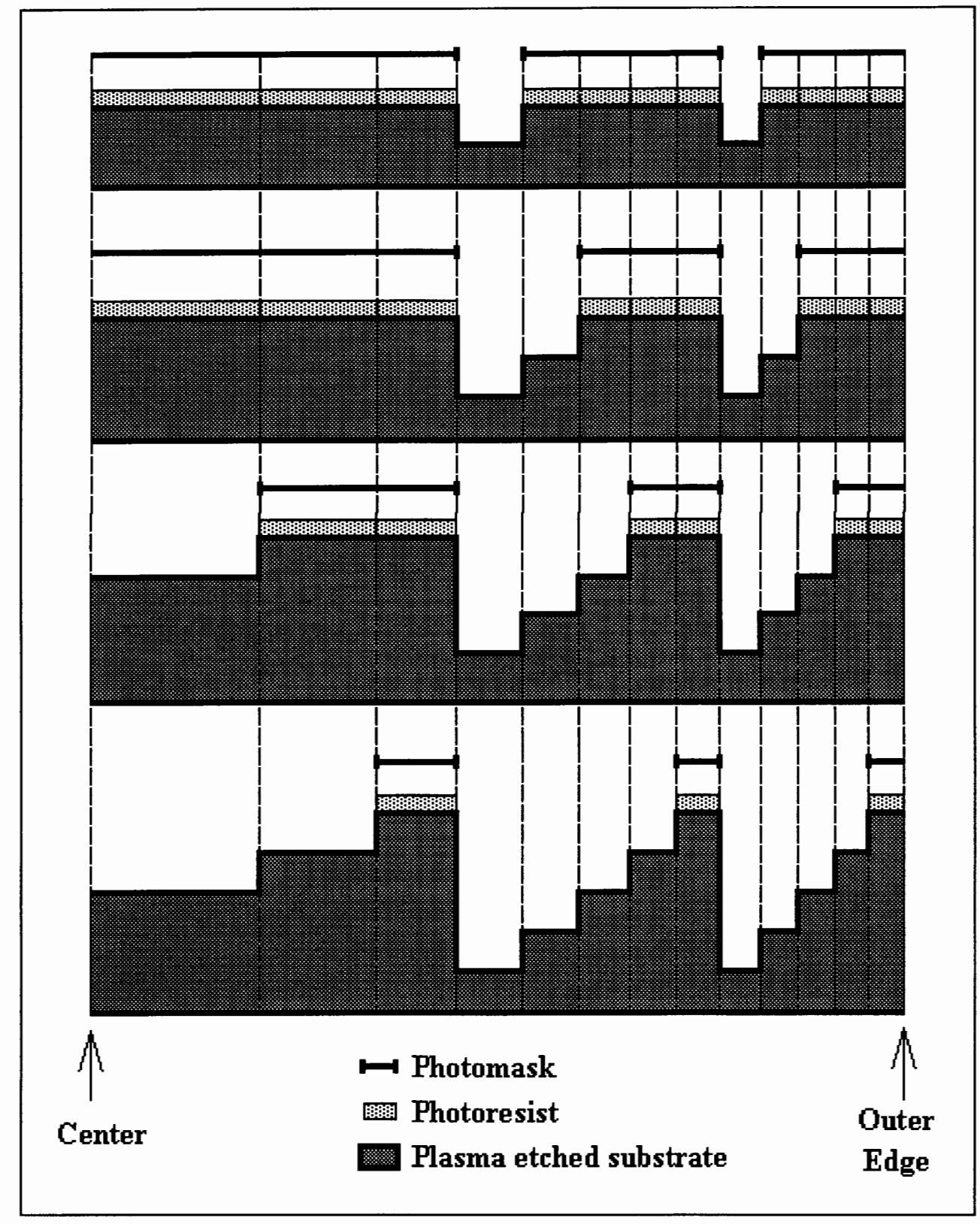

Figure 36. A process sequence, from start to finish (top to bottom), of radial cross-sections of the tier formation. A reflective type Tiered(5) Fresnel Mirror is shown.

pattern which is used to mask the etching of the substrate or some other layer. A PatternEtch cycle is required to define each tier. Therefore the cost of manufacturing is proportional to the number of tiers per zone of the Tiered Fresnel Mirror. The more tiers per zone the better the mirror performance yet the higher the cost to manufacture. The 
cost per performance must be determined prior to production since this defines the design of the Tiered Fresnel Mirror.

However, the main advantage of IC processing is the low cost per mirror realized due to batch processing where thousands of Tiered Fresnel Mirrors will be produced economically. Figure 37 shows a top view of an eight inch wafer having 185 Tiered(15) Fresnel Mirrors. Figure 38 shows a zoom-in of Figure 36 illustrating the individual tiers.

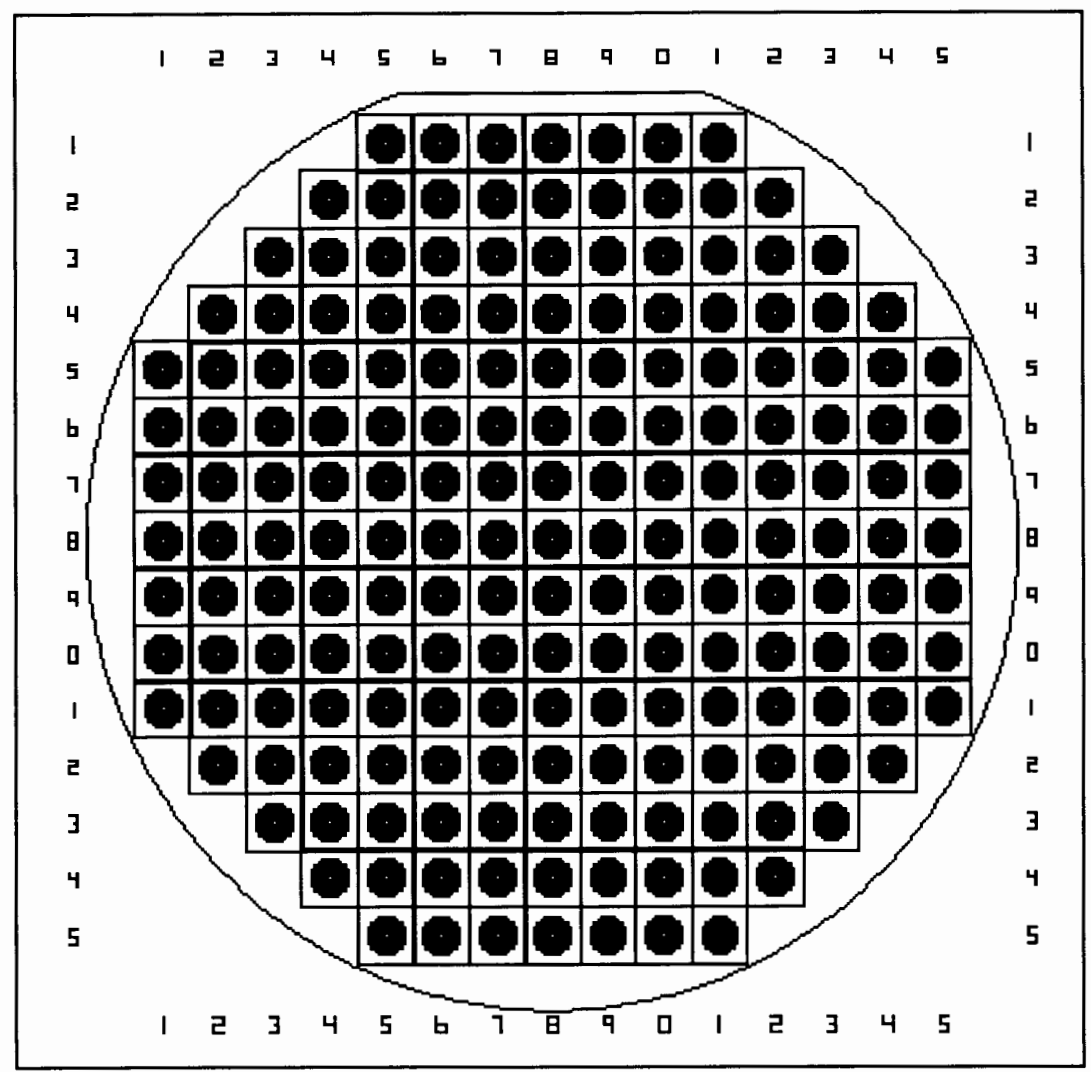

Figure 37. An eight inch wafer containing 185 Tiered(15) Fresnel Mirrors.

If a Lot of wafers consists of 20 eight inch wafers and if the yield is $90 \%$ one can obtain 3,330 Tiered(15) Fresnel Mirrors. If 10 Lots were processed one can obtain 33,300 Tiered(15) Fresnel Mirrors. So one can see that the cost per mirror decreases drastically from one wafer to a Lot of wafers. The price per Lot remains fixed. In other words, the cost to process one wafer is a little less than to process a whole Lot and the 


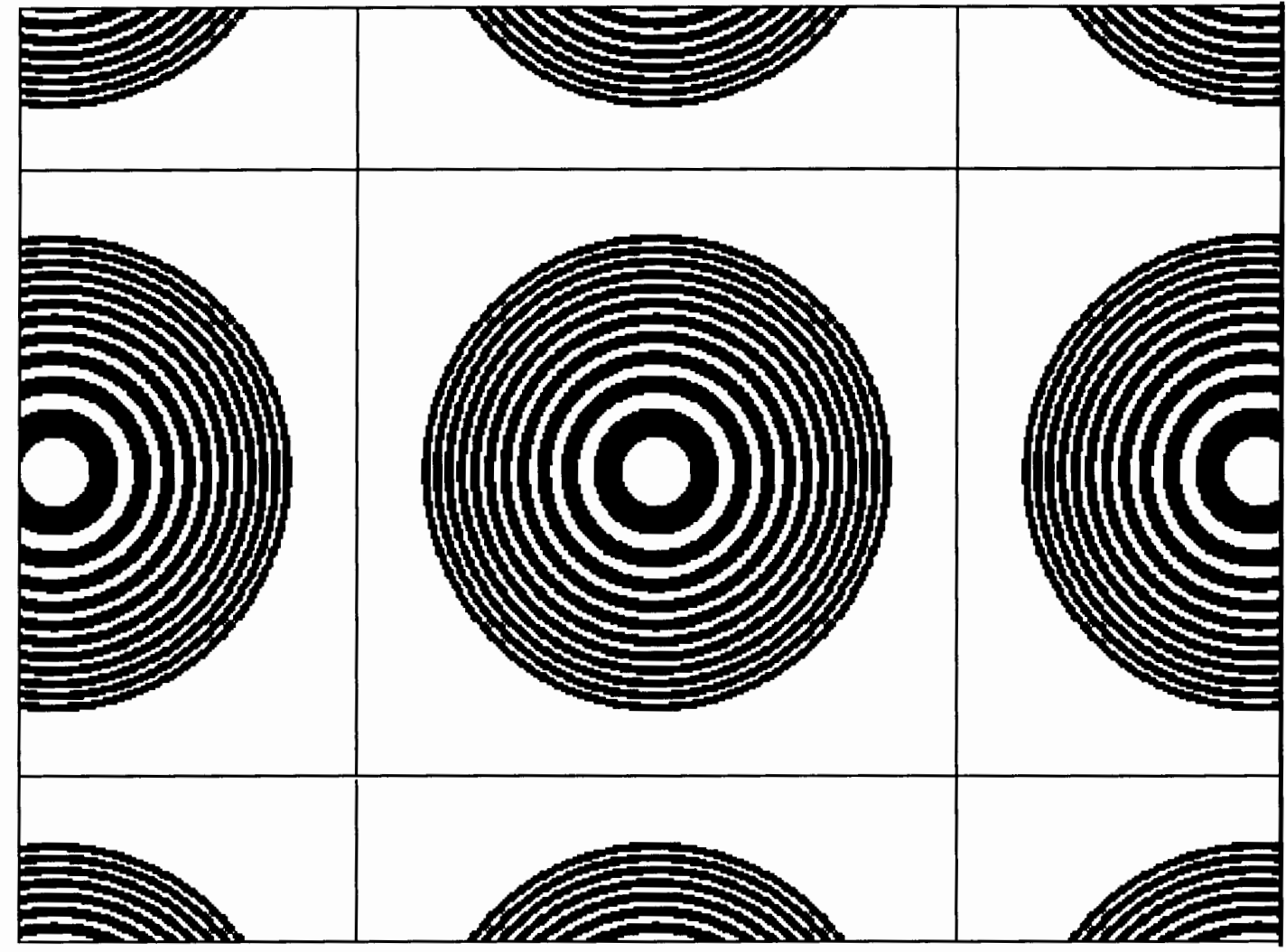

Figure 38. A zoom-in of a section of Figure 37. Notice there are 23 tiers in this three-zone Tiered(15) Fresnel Mirror.

one wafer yields say 166 mirrors whereas the Lot yields say 3,330 mirrors. The cost per mirror may be 10-20 times cheaper for the Lot compared to the single wafer.

A process simulation was performed where the variable of concern was the tier step height. A two zone Tiered(15) Fresnel Mirror was used to determine the performance in the form of a loss per pass value given in percent. A wavelength of 10.6 microns was used which determines the target tier step height at 3533 angstroms. An etch depth specification of $3533 \pm 350$ angstroms $(3 \sigma)$ was incorporated into the simulation. Three separate trial runs were compared to the ideal case where all tier step heights were equal to 3533 angstroms. Table III shows the data for this simulation. Note that the loss per pass does not vary much which says that a 350 angstrom tolerance is 
satisfactory for the performance of the mirror. $\mathrm{A} \pm 350$ angstrom tolerance range is a very doable specification range for a plasma etch process step.

TABLE III

SIMULATED TIER HEIGHTS

WITH ITS AFFECT ON LOSS PER PASS

FOR A SYMMETRIC CONFOCAL RESONATOR

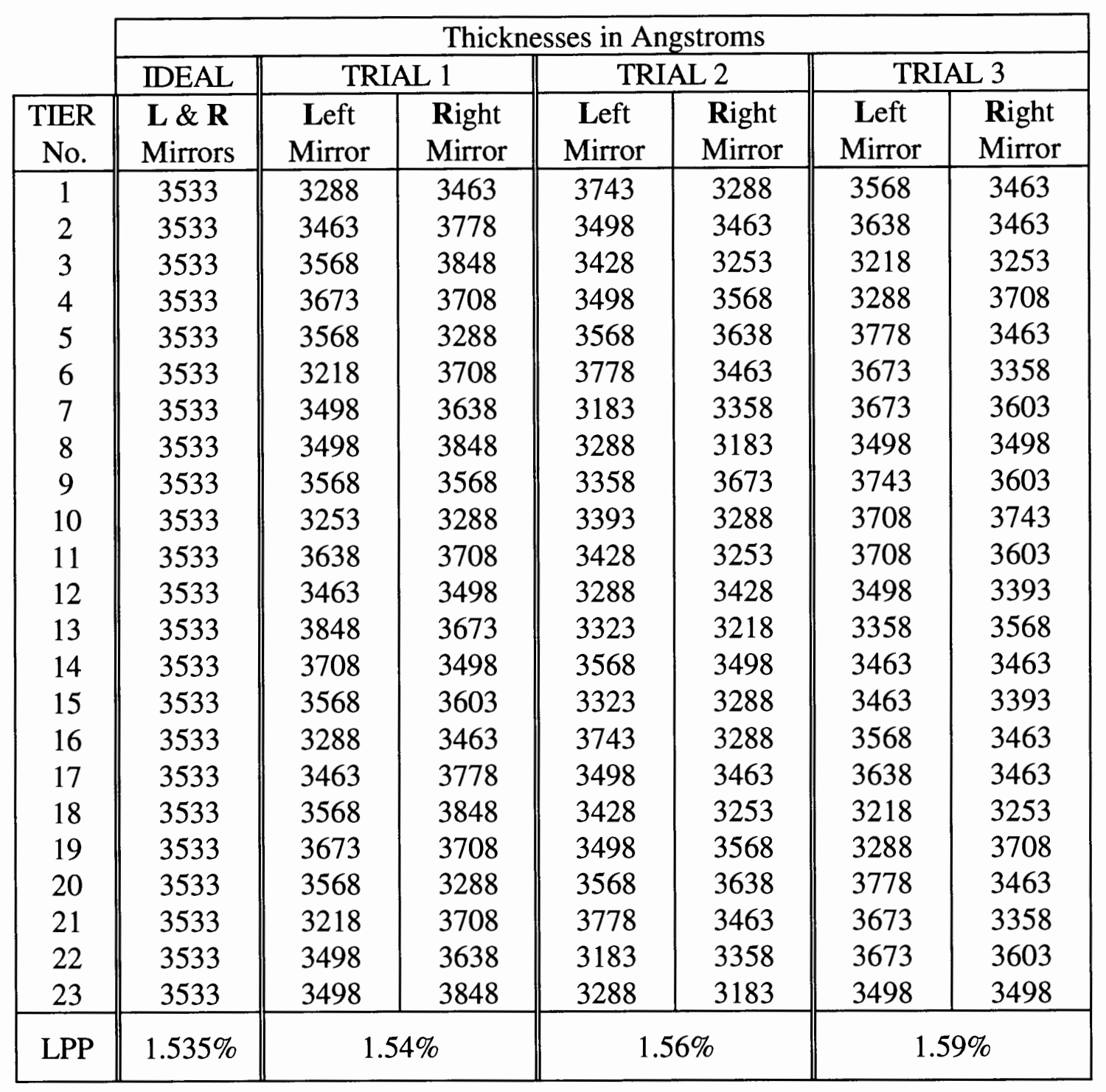




\section{CHAPTER VI}

\section{CONCLUSION}

The use of Tiered Fresnel Mirrors in Optical Resonators has been shown to be feasible. The appealling aspect of the low cost per mirror makes it desireable to produce. The Integrated Circuits Process has proven to be cost effective with Solid State Chips and for the same reason the Tiered Fresnel Mirror can be produced at a low cost per mirror. The performance was shown to be less than the Spherical Mirror yet the cost per performance ratio can be lower. In many instances one may settle for using a less efficient laser with the Tiered Fresnel Mirror depending upon the usage.

It was shown that the Fresnel Mirror acts like the Spherical Mirror and that the Tiered Fresnel Mirror is a modified Fresnel Mirror. The Tiered Fresnel Mirror can be made to act as a either a Spherical Mirror, a Plane Mirror, or somewhere between the two. How this is done is in the design, i.e. the number of tiers per zone. To emulate a Spherical Mirror one would use an infinite number of tiers per zone while on the other hand using zero tiers per zone emulates the Plane Mirror.

The Tiered Fresnel Mirror will naturally discriminate against higher transverse modes from oscillating in favor of the fundamental mode to a higher degree than that of the Spherical Mirror. With this in mind, it is easier to produce and maintain the fundamental mode and thus the TFM is an inherent mode discriminator.. Laser operation in the fundamental mode is desirable because it is generally more useful due to the beam's compact size and shape.

Finally the TFM is an inherent frequency or wavelength filter. The major variable in the design of the mirror is the wavelength from which the tier widths are determined. 
Any deviance from this wavelength causes the performance to suffer. Thus the halfwidth of the Lorentzian lineshape function may be narrowed compared to that of a conventional spherical mirror system where less discrimination of the wavelength occurs. 


\section{REFERENCES}

[1] Sobel, F., Wentworth, L. \& Wiltse C., "Quasi-optical surface waveguide and other components for the 100 to $300-\mathrm{GHz}$ region", IRE Trans. on Microwave Theory Tech., Vol MTT-9, Nov 1961, pp 512-518.

[2] Black D. \& Wiltse C., "Millimeter-Wave Characteristics of Phase-Correcting Fresnel Zone Plates", IEEE Trans.on Microwave Theory and Techniques, Vol MTT-35, Dec 1987, pp 1122-1128.

[3] Haruna M., Takahaski M., Wakahayashi K. \& Nioshihara H., "Laser Beam Lithographed Micro-Fresnel Lenses", Applied Optics, Vol 29 No. 34, 1 Dec 1990, pp 5120-5126.

[4] Nishihara H., \& Suhara T., "Micro Fresnel Lenses", Progress in Optics XXIV, E. Wolf (Elsevier Science Publishers B.V., 1987).

[5] Mottier P. \& Valette S., "Integrated Fresnel Lens on Thermally Oxidized Silicon Substrate", Applied Optics, Vol 20, No 9, 1 May 1981, pp 1630-1634.

[6] Garrett J. \& Wiltse J., "Fresnel Zone Plate Antennas at Millimeter Wavelengths", International Journal of Infrared and Millimeter Waves, Vol 12, No 3, 1991, pp 195-221.

[7] Belanger P. \& Pare C., "Optical Resonators Using Graded-Phase Mirrors", Optics Letters, Vol 16, No 14, July 15, 1991, pp 1057-1059.

[8] Born M., \& Wolf E., "Principles of Optics", (Pergamon Press: New York, NY, 6th Ed., 1980) pg 262. 
[9] Siegman, A.E., "Lasers",

(University Science Books: Mill Valley, Ca, 1986) pp581-593.

[10] Yariv, A., "Quantum Electronics",

(John Wiley \& Sons: New York, N.Y., 3rd Ed., 1989) pp 106-109.

[11] Saleh, B.E.A., \& Teich, M.C., "Fundamentals of Photonics",

(John Wiley \& Sons: New York, N.Y., 1991) pp27-30.

[12] Yariv, A., pp 142-144.

[13] Siegman, A.E., pp642-646, 686.

[14] Saleh, B.E.A., \& Teich, M.C., pp335-337.

[15] Siegman, A.E., pp647.

[16] Hecht,E. \& Zajac, A., "Optics",

(Addison-Wesley Publishing Company, Inc.: Menlo Park, Ca.,1974) pp366-372.

[17] Sussman, M., "Elementary Diffraction Theory of Zone Plates", American Journal of Physics, No. 28, 1960, pp394-398.

[18] Siegman, A.E.,pg 690.

[19] Fox, A.G., \& Li, T., "Resonant Modes in a Maser Interferometer", The Bell System Technical Journal, 1961, pp453-488.

[20] Hildebrand, F.B., "Methods of Applied Mathematics", (Prentice-Hall: Englewood Cliffs, N.J., 1952), pp 421-428. 
APPENDIX A

LISTING OF COMPUTER PROGRAM

"RESONATE VERSION 1.0" 


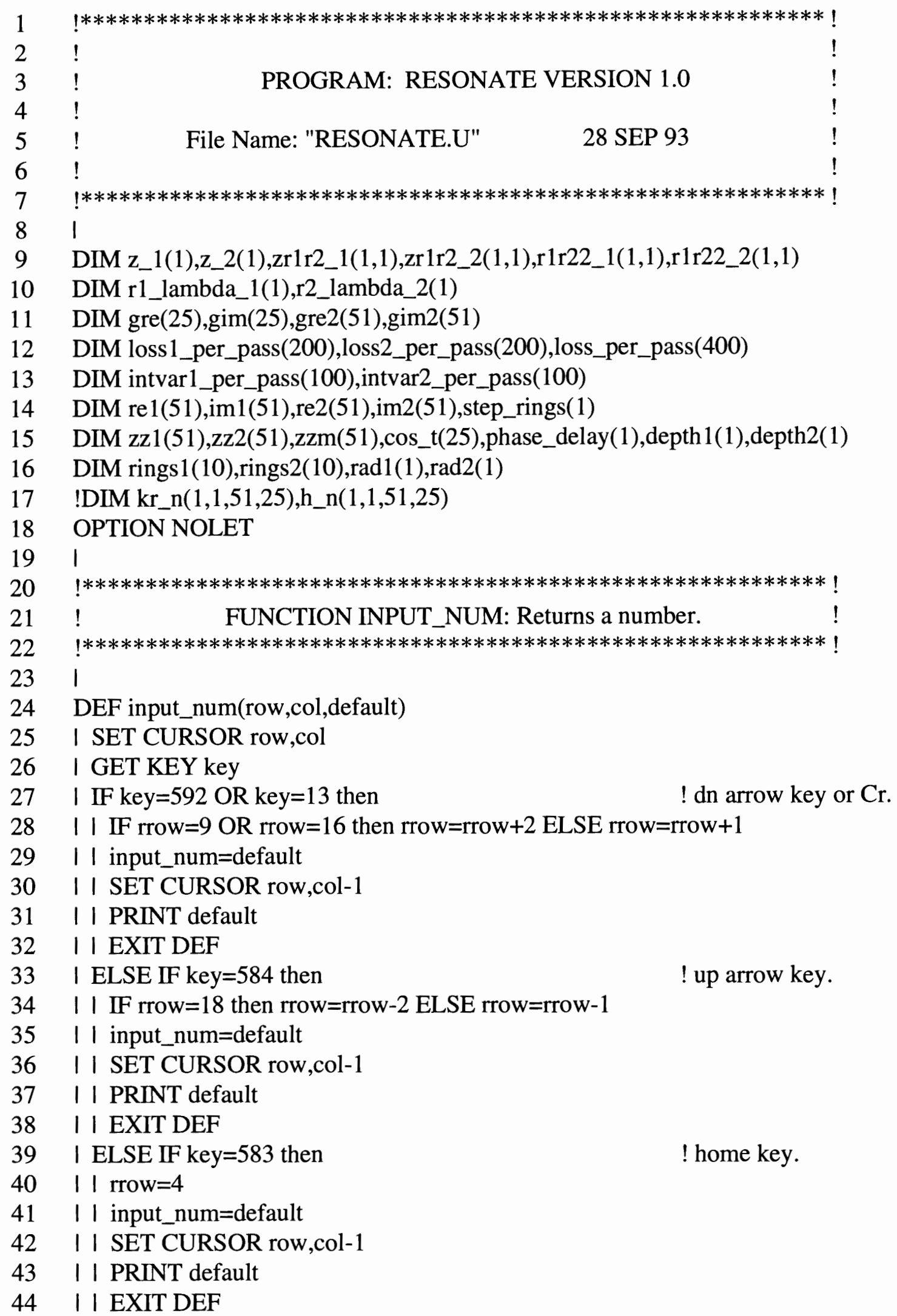


45 I ELSE IF key $=591$ then

! end key.

46 | । rrow $=24$

47 I । input_num=default

48 I I SET CURSOR row,col-1

49 I I PRINT default

50 I । EXIT DEF

$51 \mid$ END IF

52 I WHEN ERROR IN

53 I । PRINT CHR\$(key);

54 I CALL input_.

55 | 1 a $\$=$ CHR $\$($ key $) \& a$

56 I I input_num=val(a $\$$ )

57 । I IF rrow=9 OR rrow=16 then rrow=rrow +2 ELSE rrow=rrow +1

58 I USE

59 | । IF EXTYPE $=4001$ then

60 । । I SET COLOR "black/white"

61 I । I SET CURSOR 2,1

62 I । I PRINT "Invalid \# format, Cr to continue:";

63 I। I SET COLOR "white/black"

64 I I I CALL input_

65 | । I SET CURSOR 2,1

66 | | | PRINT erase_line\$

67 | || input_num=default

68 । 1 ELSE

69 I । I SET COLOR "black/white"

70 I । । SET CURSOR 2,1

71 I I I PRINT EXTYPE;EXTEXT\$;", Cr to continue:";

72 I। I SET COLOR "white/black"

73 I I C CALL input_

74 | I I SET CURSOR 2,1

75 । । I PRINT erase_line\$

76 I 1 input_num=default

77 | । END IF

78 I END WHEN

79 END DEF

801

81

82

!************************************************************!

83

$!$

FUNCTION INPUT_STRING: Returns a string.

841

85 DEF input_string $\$$ (row,col,a $1 \$, \mathrm{a} 2 \$, \mathrm{a} 3 \$, \mathrm{a} 4 \$, \mathrm{a} 5 \$$,response $\$$,default $\$$ )

86 I SET CURSOR row,col

87 I GET KEY key

88 I IF key=592 OR key=13 then

! dn arrow key or Cr. 
89 | । rrow=rrow +1

90 | $\mid$ input_string $\$=$ default $\$$

91 I I PRINT default\$

92 । । EXIT DEF

93 I ELSE IF key=584 then

94 | । IF rrow=11 then rrow=rrow-2 ELSE rrow=rrow-1

! up arrow key.

95 | 1 input_string $\$=$ default $\$$

96 I I PRINT default\$

97 । । EXIT DEF

98 I ELSE IF key=583 then

! home key.

99 I । rrow $=4$

100 | $\mid$ input_string $\$=$ default $\$$

101 | I PRINT default\$

102 | । EXIT DEF

103 | ELSE IF key=591 then

! end key.

104 | | rrow=24

10511 input_string $\$=$ default $\$$

106 I I PRINT default\$

107 । । EXIT DEF

108 I END IF

109 | WHEN ERROR IN

110 I I PRINT CHR\$(key);

111 I C CALL input_

112 | I a $\$=\mathrm{CHR} \$$ (key) \& a $\$$

113 | $\mid$ a $\$=$ UCASE $\$(\mathrm{a} \$)$

114 I I IF $a \$=a 1 \$$ OR $a \$=a 2 \$$ OR a $\$=a 3 \$$ OR $a \$=a 4 \$$ OR $a \$=a 5 \$$ then

115 | | | input_string $\$=a$

116 | | | rrow=rrow +1

117 | $\mathrm{ELSE}$

118 I I SET COLOR "black/white"

119 । । I SET CURSOR 2,1

120 I I I PRINT response\$;", Cr to continue:";

121 I I I SET COLOR "white/black"

122 I I I CALL input

123 | । | SET CURSOR 2,1

124 | | | PRINT erase_line\$

125 | | | input_string $\$=$ default $\$$

126 | | END IF

127 I USE

128 I I SET COLOR "black/white"

129 I । SET CURSOR 2,1

130 I । PRINT EXTYPE;EXTEXT\$;", Cr to continue:";

131 I I SET COLOR "white/black"

132 I I CALL input_ 
133 | I SET CURSOR 2,1

134 | | PRINT erase_line\$

135 | 1 input_string $\$=$ default $\$$

136 I END WHEN

137 END DEF

1381

139

!****************************************************************)!

140 ! SUBROUTINE INPUTS: Retains current inputs after CNTRL-BRK. !

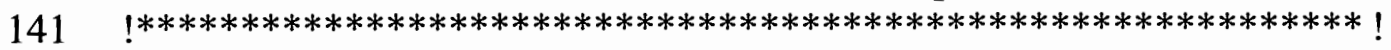

1421

143 SUB inputs

144 I inputs $\$=" Y "$

145 I CALL main

146 END SUB

147

148

149

150

1

151

152 SUB open_viewports

153 ।

154 | FOR $\mathrm{i}=1$ to 15

155 I I CLOSE \# $\mathrm{i}$

156 I NEXT i

157 | OPEN \#1 : SCREEN 0.0500,0.9500,0,1

158 I OPEN \#2 : SCREEN 0.0500,0.1100,0.45,0.8

159 I OPEN \#3 : SCREEN 0.1100,0.2753,0.45,0.8

160 I OPEN \#4 : SCREEN 0.3113,0.4766,0.45,0.8

161 I OPEN \#5 : SCREEN 0.4994,0.6647,0.45,0.8

162 I OPEN \#6 : SCREEN 0.7247,0.8900,0.45,0.8

163 I OPEN \#7 : SCREEN 0.890,0.950,0.45,0.8

164 I OPEN \#8 : SCREEN 0.110,0.338,0.08,0.4

165 I OPEN \#9 : SCREEN 0.374,0.602,0.08,0.4

166 I OPEN \#10: SCREEN 0.662,0.890,0.08,0.4

167 I OPEN \#11: SCREEN 0.110,0.338,0.04,0.07

168 I OPEN \#12: SCREEN 0.374,0.602,0.04,0.07

169 I OPEN \#13: SCREEN 0.662,0.890,0.04,0.07

170 I OPEN \#14: SCREEN 0.090,0.935,0.815,0.835

171 I OPEN \#15: SCREEN 0.05,0.95,0,0.04

!!!

172 END SUB

$173 \quad$ I

174

175

!****************************************************************!

176

SUBROUTINE INIT: Initializes the important variables.

!

$\mid * * * * * * * * * * * * * * * * * * * * * * * * * * * * * * * * * * * * * * * * * * * * * * * * * * * * * * * * * * *)$ 
$177 \quad$ ।

178 SUB init

179 I CALL open_viewports

180 I ! Mirror types: SPHERICAL, PARABOLIC, PLANE, FRESNEL, or TIERED.

181 | m1\$="SPHERICAL"

182 | m2\$="SPHERICAL"

183 | radius1,radius $2=1$

184 | zones1,zones2=3

185 | tpz1,tpz2=15

186 | outer_tiers $1=0$

187 outer_tiers $2=0$

188 | cavity_length $=1$

189 | lambda=10.6e-6

! Mirror1 type.

! Mirror2 type.

! Radii of curvature for M1,M2.

! \# of complete Fresnel zones.

190 I max_rt=200

! \# of tiers per zone. Must be ODD.

! \# of outer-zone tiers, counting from the

! center of the last zone.

! The resonator length (mirror separation).

! The wavelength of the laser light.

! Total transits ( 2 transits $=1$ round trip).

191 I incr=50 ! Radial increments. MUST BE EVEN for SUB Simpsons_Rule.

192 I inct=40 ! Theta increments. MUST BE EVEN for SUB Simpsons_Rule.

193 I input_wave $\$=" P L A N E "$ ! "PLANE" (or "GAUSSIAN"): Input wave.

194 I IF MOD $(\operatorname{tpz} 1,2)=0$ then tpz $1=t p z 1+1 \quad$ ! Odd \# of tiers per zone only.

195 I IF zones $1=0$ then

196 | | tiers1=outer_tiers1

197 I ELSE

198 | । tiers1=tpz1*(zones1-0.5)+0.5+outer_tiers1 ! Total \# of tiers.

199 I END IF

200 I max_radius1=SQR(radius $1 *(2 *$ tiers $1-2) * l a m b d a / 2 /$ tpz $1+((2 *$ tiers $1-2)$

I I *lambda/2/tpz1)^2)

201 I IF MOD $(\operatorname{tpz} 2,2)=0$ then tpz2=tpz $2+1 \quad$ ! Odd \# of tiers per zone only.

202 I IF zones $2=0$ then

203 | | tiers2=outer_tiers2

204 I ELSE

205 | | tiers2=tpz $2 *($ zones2-0.5)+0.5+outer_tiers2 ! Total \# of tiers.

206 I END IF

207 I max_radius2=SQR(radius2*(2*tiers2-2)*lambda/2/tpz2+((2*tiers2-2)

1 | *lambda/2/tpz2)^2)

208 | similate $\$=" \mathrm{~N}^{\prime}$

209 | RANDOMIZE

210 I alternate $\$=" Y "$

211 | step_switch $\$=" N "$

212 | step_tiers $=5$

213 I $\mathrm{n}=0$

214 | show $\$=" Y "$

215 I dev=7e-4 !Stops program when the amplitude fluxuations are minimal.

216 | auto_scale $\$=" Y "$ ! Y/N: Auto-scales the log plot of power loss.

217 | erase_line $\$=$ REPEAT $\$(", 77)$

218 END SUB

! Y/N: Similates a processing of the step heights.

! Used for process similation.

! Step mirrors: $\mathbf{M} 1=$ (recessed,raised) $M 2=$ vice versa.

! Step mirrors: Recessed->raised;raised->recessed.

! Step mirrors only. Total \# of rings.

! Used to maximize RAM usage. 


\section{(1)}

225 CALL main

226

227

231 SUB main

232 | IF inputs $\$=" N "$ then CALL init

233 I DO

234 | I CALL open_viewports

235 | । WINDOW \#1

236 | | CLEAR

237 | | SET COLOR "white"

238 | | CALL variable_change

239 I । IF MOD $(\operatorname{tpz} 1,2)=0$ then tpz1=tpz $1+1$

240 | | tiers $1=$ tpz $1 *$ zones 1

! Number of tiers.

241 | | IF zones $1=0$ then

242 | | | tiers1=outer_tiers1

243 | | ELSE

244 | । | tiers1=tiers1-(tpz1-1)/2+outer_tiers1

! Total tiers $=$ tiers +

245 | । END IF

246 | । IF MOD $(\operatorname{tpz} 2,2)=0$ then $\operatorname{tpz} 2=\operatorname{tpz} 2+1$

247 | | tiers2=tpz2*zones2

! Number of tiers.

248 | । IF zones $2=0$ then

249 | | | tiers2=outer_tiers2

250 | । ELSE

251 | । | tiers2=tiers2-(tpz2-1)/2+outer_tiers2

252 | । END IF

253 | । max_transits $=2 *$ max_rt

254 | । MAT REDIM z_1(incr+1),z_2(incr+1)

255 | । MAT REDIM zr1r2_1(incr+1,incr+1),zr1r2_2(incr+1,incr+1)

256 | | MAT REDIM r1_lambda_1(incr+1),r2_lambda_2(incr+1)

257 I I MAT REDIM r1r22_1(incr+1,incr+1),r1r22_2(incr+1,incr+1)

258 I । MAT REDIM rad1(zones1+1),rad2(zones2+1)

259 | । MAT REDIM rings1(tiers1),rings2(tiers2)

260 | I MAT REDIM phase_delay(incr+1),depth1(tpz1),depth2(tpz2)

261 I I MAT REDIM gre(inct+1), gim(inct+1),gre2(incr+1), gim2(incr+1)

262 I । MAT REDIM re1(incr+1),im1(incr+1), re2(incr+1), im2(incr+1) 
263 | I MAT REDIM zz1(incr+1),zz2(incr+1),zzm(incr+1),cos_t(inct+1)

264 | I MAT REDIM loss1_per_pass(max_rt),loss2_per_pass(max_rt)

265 | I MAT REDIM step_rings(step_tiers+1),loss_per_pass(max_transits)

266 I I MAT REDIM intvar1_per_pass(max_rt),intvar2_per_pass(max_rt)

267 | | !MAT REDIM kr_n(2,n+1,incr+1,inct+1),h_n $(2, \mathrm{n}+1$, incr+1, inct+1)

268 I I input_wave $\$=U C A S E \$\left(i n p u t \_w a v e \$\right)$

$269 \mid 1 \mathrm{~m} 1 \$=\mathrm{UCASE} \$(\mathrm{~m} 1 \$)$

270 | $1 \mathrm{~m} 2 \$=U C A S E \$(\mathrm{~m} 2 \$)$

271 | | alternate $\$=U C A S E \$($ alternate $\$$ )

272 | I step_switch\$=UCASE\$(step_switch\$)

273 | | auto_scale\$=UCASE\$(auto_scale\$)

274 | | show=0.845/incr

275 | | k=2*pi/lambda

276 | | IF m1\$="STEP" then

277 | | | etch_depth=lambda/4

278 | | | etch_depth\$="LAMBDA/4"

279 | | | FOR $\mathrm{i}=1$ to step_tiers

280 | | | j=2*i-1

! Find the odd lambda/4

28

I I | | step_rings $(\mathrm{i})=\mathrm{SQR}($ fzt_focus*j*lambda/2+(j*lambda/4)^2)

282 | | | NEXT i

283 | | | step_max_radius=step_rings(step_tiers)

284 | | | dr1,dr2=step_max_radius/incr

! Max mirror radius.

285 | | ELSEIF m1\$="TIERED" OR m1\$="FRESNEL" then

286 | | | FOR $\mathrm{i}=1$ to tiers 1

! Incremental radii.

287 । । । । IF $\mathrm{i}=$ tiers 1 then

288|||| $\mid \mathrm{j}=2 * \mathrm{i}-2$

289 | $|1|$ ELSE

290 | | | | | j=2*i-1

291 | | | | END IF

292||||$|\quad| \mathrm{j}=2 *^{\mathrm{i}-1}$

! Find odd lambda/ $(2 *$ tpz 1$)$ radii.

293 | | | | rings $1(\mathrm{i})=\mathrm{SQR}($ radius $1 * \mathrm{j} *$ lambda/2/tpz1+(j*lambda/2/tpz1)^2)

294 | | | NEXT i

295 | | | max_radius1=rings1(tiers1)

! Max Mirror1 radius.

296 | । | FOR $\mathrm{i}=1$ to zones 1

! The outside edge of the outermost tier.

297 | | | | $\operatorname{rad} 1(\mathrm{i})=\operatorname{SQR}\left((2 * \mathrm{i}-1) *\right.$ lambda ${ }^{*}$ adius $\left.1 / 2+((2 * \mathrm{i}-1) * \text { lambda/4 })^{\wedge} 2\right)$

298 | | | NEXT $\mathrm{i}$

299 | । I rad1(i)=max_radius1

300 । । । etch_depth1=lambda/( $2 *$ tpz1)

301 | । | etch_depth $1 \$=" W / " \&$ STR $\$(2 * \operatorname{tpz} 1)$

302 | | | tol $=700 \mathrm{e}-10$

! Tolerance of etch_depth $1=+/-700$ Angstroms.

303 | । | sim $1=$ tol $/ 10$

! Used to divide the tolerance range into 20 parts.

304 | | | $\operatorname{sim} \$=" "$

305 | | | scale $=1.5$ ! $\mathrm{A}=-$ tol, $\mathrm{B}=-0.9$ tol..., $\mathrm{J}=-0.1$ tol, $\mathrm{K}=+0.1$ tol..., $\mathrm{T}=+$ tol.

306 | | | FOR $\mathrm{i}=1$ to tpz1

! Used to convert a uniform into a normal distribution. 
307 | | | | IF similate $\$=" N "$ then

308 | | | | | depth1(i)=etch_depth1*MOD $((\operatorname{tpz} 1-3) / 2+\mathrm{i}, \mathrm{tpz} 1)$

309 | | | | ELSE

310 | | | | random=10* rnd

311 | | | | IF random $<5$ then

312 | | | | | sim=tol/scale*(scale-LOG(1+random*(EXP(scale)-1)/5))

313|||||| $\operatorname{sim}=-\operatorname{sim} 1 * \operatorname{INT}(1+\operatorname{sim} / \operatorname{sim} 1)$

! - deviance from

314|||||| $\mathrm{s} \$=\mathrm{CHR} \$(75+\mathrm{INT}(\operatorname{sim} / \operatorname{sim} 1))$

! mean (thinner).

315 | | | | ELSE

316 | | | | | random=10-random

317 | | | | | sim=tol/scale*(scale-LOG(1+random*(EXP(scale)-1)/5))

318 | | | | | $\operatorname{sim}=\operatorname{sim} 1 * \operatorname{INT}(1+\operatorname{sim} / \operatorname{sim} 1)$

319 | | | | | s\$=CHR $\$(74+\mathrm{INT}(\operatorname{sim} / \operatorname{sim} 1))$

$!+$ deviance from

320 | 1111 END IF

321 | | | | $\operatorname{sim} \$=\operatorname{sim} \$ \& \mathrm{~s} \$$

322 | | | | | depth1(i)=etch_depth $1 * \operatorname{MOD}((\operatorname{tpz} 1-3) / 2+\mathrm{i}, \operatorname{tpz} 1)+\operatorname{sim}$

323 | । | | END IF

324 | । N NEXT i

325 । । END IF

326 | | dr1=max_radius $1 /$ incr

! Incremental radius: dr1 for Mirror1.

327111

328 | । IF m2\$="STEP" then

329 | | | etch_depth=lambda/4

330 | | | etch_depth\$="LAMBDA/4"

331 | | | FOR $i=1$ to step_tiers

332|||| $\mathrm{j}=2 * \mathrm{i}-1$

! Find the odd lambda/4

333 | | | | step_rings(i)=SQR(fzt_focus $\left.* j * l a m b d a / 2+(j * l a m b d a / 4)^{\wedge} 2\right)$

334 I I I NEXT i

335 | | | step_max_radius=step_rings(step_tiers) ! Max mirror radius.

336 | | | dr1,dr2=step_max_radius/incr ! Incremental radii.

337 | । ELSEIF m2\$="TIERED" OR m2\$="FRESNEL" then

338 | | | FOR $i=1$ to tiers2

! Find odd lambda/(2*tpz2) radii.

339 | | | | IF $\mathrm{i}=$ tiers 2 then

340|||| $\mid \mathrm{j}=2 * \mathrm{i}-2$ ! The outer edge of the outermost tier.

341 | । | | ELSE

342|||| $\mid \mathrm{j}=2 *_{\mathrm{i}-1}$

343 | $|1|$ END IF

344|||| $\mid ! j=2 *_{\mathrm{i}-1} \quad$ ! The center of the outermost tier.

345 | | | | rings $2(\mathrm{i})=\mathrm{SQR}\left(\operatorname{radius} 2 * \mathrm{j} * \operatorname{lambda} / 2 / \mathrm{tpz} 2+(\mathrm{j} * \operatorname{lambda} / 2 / \mathrm{tpz} 2)^{\wedge} 2\right)$

346 | I | NEXT i

347 | | | max_radius2=rings2(tiers2)

! Max Mirror1 radius.

348 | | | FOR $\mathrm{i}=1$ to zones2

Radii of Fresnel zones.

349|||| $\operatorname{rad} 2(\mathrm{i})=\operatorname{SQR}\left((2 * \mathrm{i}-1) * \operatorname{lambda} * \operatorname{radius} 2 / 2+((2 * \mathrm{i}-1) * \operatorname{lambda} / 4)^{\wedge} 2\right)$

350 | | | NEXT i 
351 | । । $\operatorname{rad} 2(\mathrm{i})=$ max_radius 2

352 | । । etch_depth2=lambda/( $2 *$ tpz 2$)$

353 | । । etch_depth $2 \$=" W / "$ \& STR $\$(2 * t p z 2)$

354 | । । tol=700e-10

! Tolerance of etch_depth $2=+/-700$ Angstroms.

355 | । । $\operatorname{sim} 1=\mathrm{tol} / 10$

! Used to divide the tolerance range into 20 parts.

356 । । । $\operatorname{sim} \$=" "$ ! $\mathrm{A}=-$ tol, $\mathrm{B}=-0.9$ tol..., $\mathrm{J}=-0.1$ tol, $\mathrm{K}=+0.1$ tol..., $\mathrm{T}=+$ tol.

357 | | | scale $=1.5$ ! Used to convert a uniform into a semi-normal

358 | | | FOR $\mathrm{i}=1$ to tpz2

distribution.

359 । । । । IF similate $\$=" N "$ then

360 |। |। $\mid$ depth2(i)=etch_depth $2 * \operatorname{MOD}((\operatorname{tpz} 2-3) / 2+\mathrm{i}$, tpz 2$)$

361 | । | | ELSE

362 | । | । | random $=10 *$ rnd

$363 \mid$ | | | | IF random $<5$ then

364 | | | | | sim=tol/scale*(scale-LOG(1+random*(EXP(scale)-1)/5))

365 | । | । | $\operatorname{sim}=-\operatorname{sim} 1 * \operatorname{INT}(1+\operatorname{sim} / \operatorname{sim} 1)$

366 | | | || $\$$ \$ $=$ CHR $\$(75+$ INT $(\operatorname{sim} / \mathrm{sim} 1))$

! - deviance from

367 | । | | | ELSE

368 | | | | | random=10-random

369 |।। | । sim=tol/scale*(scale-LOG(1+random*(EXP(scale)-1)/5))

370 | | | | | $\operatorname{sim}=\operatorname{sim} 1 * \operatorname{INT}(1+\operatorname{sim} / \operatorname{sim} 1)$

371 | | | || $\$ \$=C H R \$(74+\mathrm{INT}(\operatorname{sim} / \mathrm{sim} 1))$

$!+$ deviance from

372 | 1 | | | END IF

373 | $1|1| \operatorname{sim} \$=\operatorname{sim} \$ \& \mathrm{~s} \$$

374 |।।। | depth2(i)=etch_depth $2 * \operatorname{MOD}((\operatorname{tpz} 2-3) / 2+\mathrm{i}$, tpz 2$)+\operatorname{sim}$

375 । । । I END IF

376 | । | NEXT i

377 | । END IF

378 | । dr2=max_radius $2 /$ incr

! Incremental radius: $\mathrm{dr} 2$ for Mirror2.

379111

380 | | dt=pi/inct

! Incremental theta.

381 | । FOR $\mathrm{i}=0$ to inct

382 | । | cos_t $(\mathrm{i}+1)=\cos \left(\mathrm{i}^{*} \mathrm{dt}\right)$

383 | | NEXT $\mathrm{i}$

384 | । ।

385 । । WINDOW \#2

386 | | IF max_radius1 $\Rightarrow$ max_radius2 then

! Plot mirror1.

387 | | | bot_top=incr

388 | । ELSE

389 | | | bot_top=max_radius $2 /$ max_radius $1 *$ incr

390 | । END IF

391 I I IF radius $1>0$ then

392 | । । left $=-0.15$

393 | । | right $=0.05$

394 | | | flood_cent $=-0.125$ 


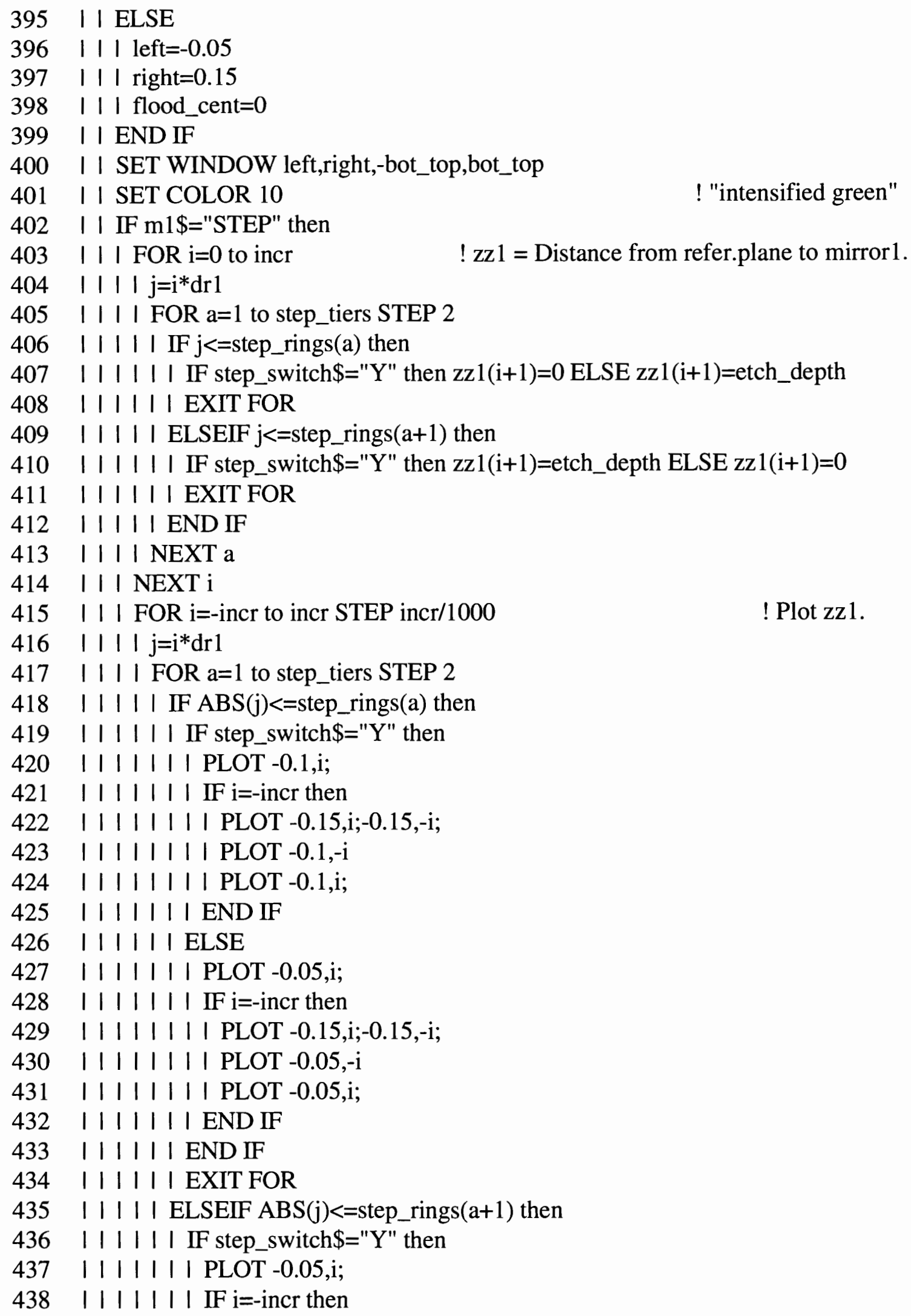


439 । |। |। । | PLOT -0.15,i;-0.15,-i;

440 | ||| | || PLOT $-0.05,-$ i

441|||||||| PLOT -0.05 ,i;

442 | |||||| END IF

$443|1||| \mid$ ELSE

444 | | | | | | PLOT -0.1,i;

445 | |||||| IF i=-incr then

446 |।|।|।|| PLOT $-0.15, \mathrm{i} ;-0.15,-\mathrm{i}$;

447 | | | | | | PLOT $-0.1,-$ i

$448 \quad||||||||$ PLOT - 0.1, ;

449 | $|1|||||$ END IF

$450|1| 1||$ END IF

451 | । । | | EXIT FOR

452 | |||| END IF

453 | | | | NEXT a

454 I । I NEXT $\mathrm{i}$

455 | | ELSEIF $\mathrm{m} 1 \$=$ "PLANE" then

456 | | | MAT zz1=0

457 । । । PLOT -0.05,incr;-0.05,-incr;-0.15,-incr;-0.15,incr;-0.05, incr

458 | । ELSEIF $m 1 \$=" T I E R E D "$ then

459 | । | FOR $i=0$ to incr

460 | | | | j=i*dr1

461 | | | | FOR $a=1$ to tiers 1

462 | |||| IF $j<=$ rings 1 (a) then

463 | । । । । CALL DIVIDE(a,tpz1,q,q1)

464 | । | | | | IF q1 $=0$ then $q 1=$ tpz 1

465 | | | | | | zzl(i+1)=depth1(q1)

466 | । $\mid$ । । EXIT FOR

467 । $|1|$ END IF

468 । । । । NEXT a

469 | | | NEXT i

470 | | | FOR $\mathrm{i}=$-incr to incr STEP incr/1000

471 | | | $\mathrm{j}=\mathrm{i}^{*} \mathrm{dr} 1$

472 | । | | FOR $a=1$ to tiers 1

473 । । । । IF ABS $(j)<=$ rings $1(a)$ then

474 । । । । । CALL DIVIDE(a,tpz1,q,q1)

475 । । । । । । IF q1=0 then $q 1=$ tpz1

476 । । । । । PLOT $-0.1 *(1-\operatorname{depth} 1(\mathrm{q} 1) / \operatorname{depth} 1((\operatorname{tpz} 1+1) / 2))$,i;

477 | | | | | | IF $\mathrm{i}=$-incr then

478 । $|।|||$ PLOT -0.15 , i;-0.15,-i;

479 । । । । |। PLOT $-0.1 *(1-\operatorname{depth} 1(\mathrm{q} 1) / \operatorname{depth} 1((\operatorname{tpz} 1+1) / 2))$,-i

480 । । । । । । PLOT $-0.1 *(1-$ depth1(q1)/depth1((tpz1+1)/2)),i;

481 | |||| $\mid$ END IF

482 | |||| EXIT FOR 


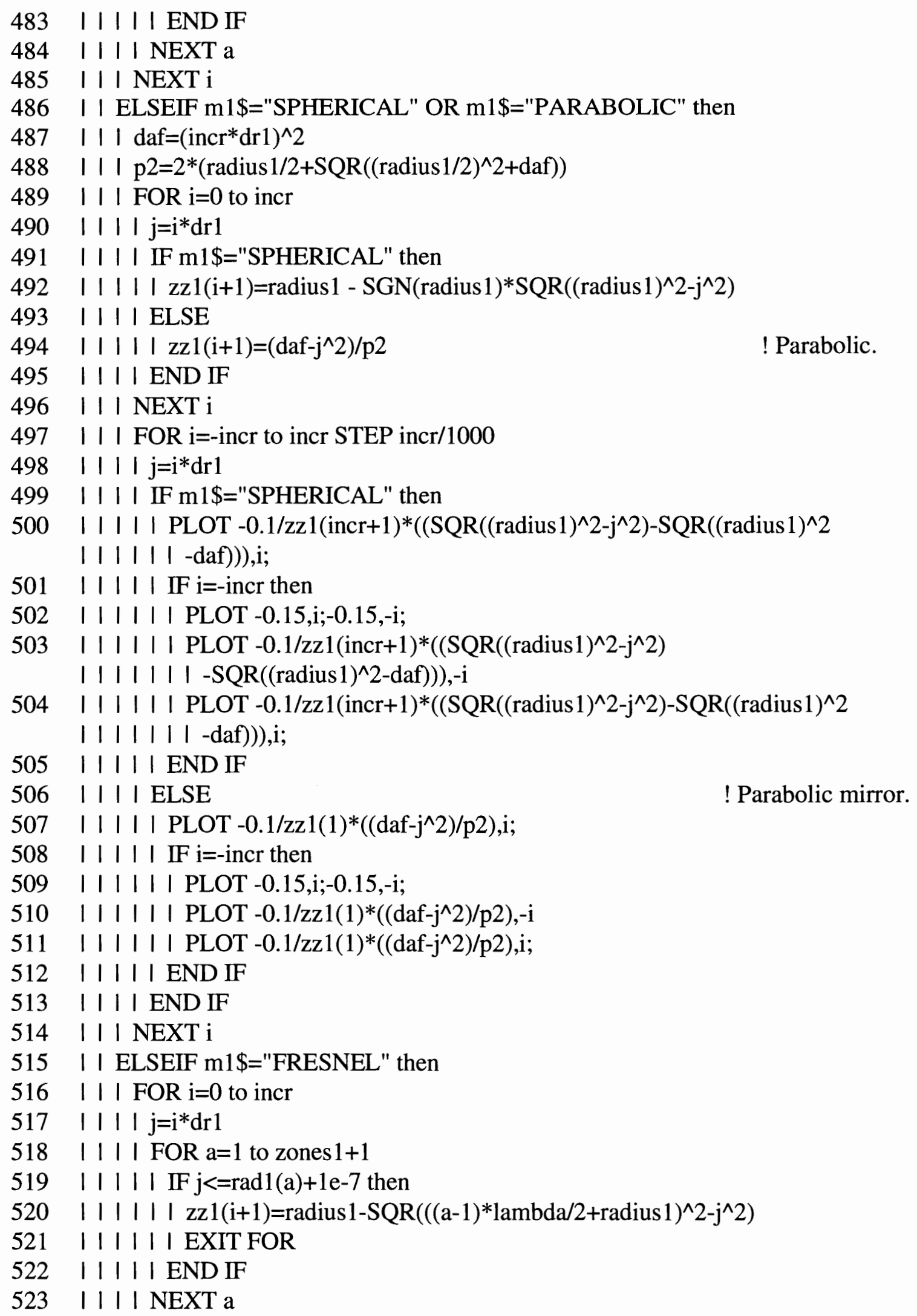


524 | | | NEXT i

525|| $\mid \mathrm{z} 1=100$

526|| $\mid z 11=-100$

527 | | | FOR $\mathrm{i}=1$ to incr+1

528||||$z 1=\min (\mathrm{z} 1, \mathrm{zz} 1(\mathrm{i}))$

529||||$z 11=\max (z 11, z z 1(i))$

530|| $\mid$ NEXT i

531 | | MAT zzm $=(-z 1) * \operatorname{con}($ incr +1$)$

532 | | MAT $z z 1=z z m+z z 1$

533|| $\mid z 1=100$

534|| $\mid z 11=-100$

535 | | | FOR $i=0$ to incr STEP incr/1000

536||||$j=i * d r 1$

537|||| FOR $a=1$ to zones $1+1$

538|||| $\mid$ IF $\mathrm{j}<=\operatorname{rad} 1(\mathrm{a})+1 \mathrm{e}-7$ then

539|||||| $\mathrm{ii}=\operatorname{radius} 1-\mathrm{SQR}\left(\left((\mathrm{a}-1)^{*} \operatorname{lambda} / 2+\operatorname{radius} 1\right)^{\wedge} 2-\mathrm{j}^{\wedge} 2\right)$

540||||||$z 1=\min (z 1$, ii $)$

541|||||| $\mathrm{z} 11=\max (\mathrm{z} 11, \mathrm{ii})$

$542|1| 1 \mid 1$ EXIT FOR

$543|1| 1 \mid$ END IF

544|||| NEXT a

545 | | | NEXT i

546 | | | FOR $i=-$ incr to incr STEP incr/1000

547|||| $\mathrm{j}=\mathrm{i} * \mathrm{dr} \mathbf{1}$

548 | | | | FOR $a=1$ to zones $1+1$

549|||| $\mid$ IF ABS(j)<=rad1(a)+1e-7 then

550 | | | | | PLOT -0.1*(1-(radius 1 - (SQR (((a-1)*lambda/2+radius1)^2-j^2)

| | | | | | | )-z1)/(z11-z1)), i;

551 | | | | | IF i=-incr then

552|||||| $\mid$ PLOT $-0.15, \mathrm{i} ;-0.15,-\mathrm{i}$;

553|||||| $\mid$ PLOT $-0.1 *\left(1-\left(\right.\right.$ radius $1-\left(\operatorname{SQR}\left(((a-1) * \operatorname{lambda} / 2+\operatorname{radius} 1)^{\wedge} 2\right.\right.$

| | | | | | | | -j^2))-z1)/(z11-z1)),-i

554|||||| $\mid$ PLOT $-0.1 *\left(1-\left(\right.\right.$ radius $1-\left(\operatorname{SQR}\left(((a-1) * \operatorname{lambda} / 2+\operatorname{radius} 1)^{\wedge} 2\right.\right.$

| | | | | | | - -j^2))-z1)/(z11-z1)), i;

$555|1||| \mid$ END IF

556|||||| EXIT FOR

557 | $11 \mid 1$ END IF

558 | | | | NEXT a

559 | | | NEXT i

560 I I END IF

561 | | FLOOD flood_cent,0

562 | | PLOT

563 | ।

564 | | WINDOW \#7

! Plot mirror2. 
565 | | IF max_radius2 $\Rightarrow>$ max_radius1 then

566 | | | bot_top=incr

567 | । ELSE

568 | | | bot_top=max_radius $1 /$ max_radius $2 *$ incr

569 | | END IF

570 | | IF radius $2>0$ then

571 । । । left $=0.05$

572 | | | right $=-0.15$

573 | | | flood_cent $=-0.125$

574 | । ELSE

575 | | | left $=0.15$

576 । । । right $=-0.05$

577 | | | flood_cent $=0$

578 | | END IF

579 I I SET WINDOW left,right,-bot_top,bot_top

580 | | SET COLOR 11

! "intensified cyan"

581 | । IF m2\$="STEP" then

582 | । | FOR $\mathrm{i}=0$ to incr $\quad ! \mathrm{zz} 2=$ Distance from refer. plane to mirror2.

583 | | | $\mathrm{j}=\mathrm{i} * \mathrm{dr} 2$

584|||| FOR a=1 to step_tiers STEP 2

585 | | | | | IF j $<=$ step_rings(a) then

586 | | | | | IF (step_switch\$="Y" and alternate\$="Y") OR (step_switch\$="N"

$111111 \mid$ and alternate $\$=" N ")$ then $z z 2(i+1)=$ etch_depth ELSE $z z 2(i+1)=0$

587 । $|1| 1 \mid$ EXIT FOR

588 | |||| | ELSEIF $j<=$ step_rings $(a+1)$ then

589 | । | | | IF (step_switch $\$=" Y$ " and alternate $\$=" Y ")$ OR (step_switch $\$=" N "$

। $|1|||$ and alternate $\$=" N "$ ) then $z z 2(i+1)=0$ ELSE zz2(i+1)=etch_depth

590 । $\mid$ । || EXIT FOR

591 | 11|| END IF

592 | । I । NEXT a

593 | | | NEXT i

594 | । | FOR $\mathrm{i}=-$-incr to incr STEP incr/1000 ! Plot zz2.

$595 \quad|||| \mathrm{j}=\mathrm{i} * \mathrm{dr} 2$

596 | || $\mid$ FOR $a=1$ to step_tiers STEP 2

597 | $1 \mid$ | | IF ABS(j)<=step_rings(a) then

598 | । । । । I IF (step_switch $\$=" Y$ " and alternate $\$=" Y "$ ) OR (step_switch $\$=" N "$

$1|1| 1 \mid 1$ and alternate $\$=" N "$ ) then

599 | | | | | | | PLOT -0.05,i;

600 | | | | | | IF i=-incr then

601 | |||||| PLOT $-0.15, \mathrm{i} ;-0.15,-\mathrm{i}$;

602 | |||||| PLOT $-0.05,-$ i

603|||||||| PLOT -0.05, i;

604|||||| $\mid$ END IF

605|||||| ELSE 
606 । $|1||| \mid$ PLOT -0.1,i;

607 | | | | | | | IF i=-incr then

608 | |||||| $\mid$ PLOT -0.15, i; -0.15,-i;

609 | |||||| $\mid$ PLOT $-0.1,-\mathrm{i}$

610 | |||||| $\mid$ PLOT - 0.1 ,i;

611 | $|1||| \mid$ END IF

612|||||| END IF

613 | |||| $\mid$ EXIT FOR

614|||| $\mid$ ELSEIF ABS $(j)<=$ step_rings $(a+1)$ then

615 | । । । IF (step_switch\$="Y" and alternate $\$=" Y "$ ) OR (step_switch $\$=" N "$

1 । 1111 and alternate $\$=" N "$ ) then

616 ||||||| PLOT -0.1,i;

617 | $|1|||$ IF $i=-$-incr then

618 |||||||| PLOT -0.15, i; $-0.15,-\mathrm{i}$;

619 | $|1||| \mid$ PLOT $-0.1,-$ i

620 |||||||| PLOT - 0.1, i;

$621|1| 1|1|$ END IF

$622|1| 1||$ ELSE

623 | $|1||| \mid$ PLOT -0.05 ,i;

$624|1| 1|| \mid$ IF i=-incr then

625 | $|1|||||$ PLOT -0.15, i; $-0.15,-$;

626 | |||||| PLOT $-0.05,-\mathrm{i}$

627 । $11|1| \mid$ PLOT -0.05 ,i;

$628111111 \mid$ END IF

$629|1||| \mid$ END IF

630111111 EXIT FOR

631 | $|1| \mid$ END IF

632 | $\mid$ | | NEXT a

633 | । | NEXT i

634 | | ELSEIF m2\$="PLANE" then

635 । । । MAT zz2=0

636 I । । PLOT -0.05,incr;-0.05,-incr;-0.15,-incr;-0.15,incr;-0.05,incr

637 | । ELSEIF $m 2 \$=" T I E R E D "$ then

638 | | | FOR $i=0$ to incr

639 | | | $\mathrm{j}=\mathrm{i} * \mathrm{dr} 2$

640 | । | | FOR $a=1$ to tiers2

641|||| $\mid$ IF $j<=$ rings2(a) then

642 | | | | | CALL DIVIDE(a,tpz2,q,q1)

! q1=remainder of $\mathrm{a} / \mathrm{tpz} 2$.

643 । $\mid$ । || $\mid$ IF q1=0 then $\mathrm{q} 1=$ tpz2

644 । $|।|||$ zz2(i+1)=depth2(q1)

645 | $|1| \mid$ EXIT FOR

646 । $|1| \mid$ END IF

647 1 1 । 1 NEXT a

648 | | | NEXT i 
649 | । | FOR i=-incr to incr STEP incr/1000

650 | || $\mid \mathrm{j}=\mathrm{i} * \mathrm{dr} 2$

651 | || FOR $a=1$ to tiers2

652 । । । | IF ABS $(j)<=$ rings $2(a)$ then

653 | । | | | CALL DIVIDE(a,tpz2,q,q1)

654 | । । । | । IF q1=0 then $\mathrm{q} 1=$ tpz2

655 |।।। । PLOT $-0.1 *(1-\operatorname{depth} 2(q 1) / \operatorname{depth} 2((\operatorname{tpz} 2+1) / 2))$,i;

656 । 1111 IF $\mathrm{i}=$-incr then

657 | |||| $\mid$ PLOT -0.15, ; $;-0.15,-$;

658 |।।।|।| PLOT $-0.1 *(1-\operatorname{depth} 2(\mathrm{q} 1) / \operatorname{depth} 2((\operatorname{tpz} 2+1) / 2)),-\mathrm{i}$

659 ।।।।।। । PLOT $-0.1 *(1-$ depth2(q1)/depth2((tpz2+1)/2)),i;

$6601|1| 1 \mid$ END IF

661 | | | | | EXIT FOR

$662|1|||$ END IF

663 | । | | NEXT a

664 | | | NEXT i

665 | । ELSEIF $m 2 \$==$ SPHERICAL" OR m2 $\$=" P A R A B O L I C "$ then

666 I। $\mid$ daf $=(\text { incr*dr2 } 2)^{\wedge} 2$

667 । । । p2 $=2 *\left(\operatorname{radius} 2 / 2+\mathrm{SQR}\left((\operatorname{radius} 2 / 2)^{\wedge} 2+\right.\right.$ daf $\left.)\right)$

668 | | | FOR $\mathrm{i}=0$ to incr

669 | || $\mid \mathrm{j}=\mathrm{i} * \mathrm{dr} 2$

670 | 11 । IF $\mathrm{m} 2 \$="$ SPHERICAL" then

$671|1||| \mathrm{zz} 2(\mathrm{i}+1)=$ radius $2-\mathrm{SGN}(\text { radius } 2)^{*} \mathrm{SQR}\left((\text { radius } 2)^{\wedge} 2-\mathrm{j}^{\wedge} 2\right)$

672 | | | | ELSE

! Parabolic.

673 | |||| zz2 $(\mathrm{i}+1)=\left(\right.$ daf $\left.-\mathrm{j}^{\wedge} 2\right) / \mathrm{p} 2$

674 । $\mid$ । $\mid$ END IF

675 | | | NEXT i

676 | । | FOR $i=-$ incr to incr STEP incr $/ 1000$

$677 \quad|1| \mid \mathrm{j}=\mathrm{i} * \mathrm{dr} 2$

678 | | | | IF m2\$="SPHERICAL" then

679 । $1 । \mid$ PLOT $-0.1 /$ zz2(incr+1)*((SQR((radius2)^2-j^2)-SQR((radius2)^2

| | | | | | -daf))), i;

680 | |||| IF i=-incr then

681 । $\mid$ । || PLOT -0.15, i; $-0.15,-\mathrm{i}$;

682|||||| PLOT $-0.1 /$ zz2(incr+1)*((SQR((radius2)^2-j^2)-SQR((radius2)^2

| $1111 \mid 1$-daf))),-i

683 | $11 \mid 1$ PLOT $-0.1 / \mathrm{zz} 2\left(\right.$ incr+1)*((SQR $\left((\text { radius2 })^{\wedge} 2-\mathrm{j}^{\wedge} 2\right)-\mathrm{SQR}\left((\text { radius2 })^{\wedge} 2\right.$

| | | | | | -daf))), i;

684 । $11 \mid 1$ END IF

685 | || $\mid$ ELSE

! Parabolic.

686 | । । । । PLOT $-0.1 /$ zz1(1)*((daf-j^2)/p2),i;

687 | $|1| \mid$ IF $\mathrm{i}=-$-incr then

688 । $\mid$ । । । PLOT $-0.15, \mathrm{i} ;-0.15,-\mathrm{i}$;

689 | |||।| PLOT $-0.1 /$ zz1 $(1)^{*}\left(\left(\right.\right.$ daf $\left.\left.-j^{\wedge} 2\right) / p 2\right),-i$ 
690 | | | | | PLOT $-0.1 / \mathrm{zz} 1(1)^{*}\left(\left(\mathrm{daf}^{\mathrm{j}} \mathrm{j}^{\wedge} 2\right) / \mathrm{p} 2\right), \mathrm{i}$;

$6911111 \mid$ END IF

692 | 1|| END IF

693 | | | NEXT i

694 | | ELSEIF m2\$="FRESNEL" then

695 | | FOR $\mathrm{i}=0$ to incr

$696|1| \mid j=i^{*} \mathrm{dr} 2$

697 | | | FOR $\mathrm{a}=1$ to zones $2+1$

698|||| $\mid$ IF $j<=\operatorname{rad} 2(a)+1 e-7$ then

$699|1| 1|| \mathrm{zz} 2(\mathrm{i}+1)=$ radius $2-\operatorname{SQR}\left(((\mathrm{a}-1) * 1 \text { ambda/2+radius } 2)^{\wedge} 2-\mathrm{j}^{\wedge} 2\right)$

700 | $11 \mid 1$ EXIT FOR

$701|1| 1 \mid$ END IF

702 | $11 \mid$ NEXT a

703 | | | NEXT $\mathrm{i}$

704 | $\mid$ | $22=100$

705 | $\mid \mathrm{z} 22=-100$

706 | | FOR $\mathrm{i}=1$ to incr+1

707 | | | z2=min(z2,zz2(i))

708 | | | $\mathrm{z} 22=\max (\mathrm{z} 22, \mathrm{zz} 2(\mathrm{i}))$

709 | | | NEXT i

710 | | | MAT zzm=(-z2)*con $($ incr +1$)$

711 । । MAT $z z 2=z z m+z z 2$

712|| $\mid \mathrm{z} 1=100$

713 | | $\mathrm{z} 11=-100$

714|| $\mid$ FOR $i=0$ to incr STEP incr $/ 1000$

715 | ||$j=i * d r 2$

716 | | | | FOR $\mathrm{a}=1$ to zones $2+1$

717 | | | | IF $j<=\operatorname{rad} 2(a)+1 e-7$ then

718 | | | | | ii=radius2 - SQR(((a-1)*lambda/2+radius2)^2-j^2)

719 | | | | | z1=min(z1,ii)

720||||||$z 11=\max (\mathrm{z} 11, \mathrm{ii})$

$721|1||| \mid$ EXIT FOR

722 | $11 \mid 1$ END IF

723 | | | I NEXT a

724 | | | NEXT i

725 | | | FOR $\mathrm{i}=$-incr to incr STEP incr/1000

726 | $\mid 1 \mathrm{j}=\mathrm{i}^{*} \mathrm{dr} 2$

727 | | | | FOR a=1 to zones $2+1$

$728 \quad|1|||$ IF ABS $(j)<=\operatorname{rad} 2(a)+1 e-7$ then

729|||||| PLOT $-0.1^{*}\left(1\right.$-(radius2 - $\left(\operatorname{SQR}\left(\left((a-1)^{*} \text { lambda/2+radius } 2\right)^{\wedge} 2-j^{\wedge} 2\right)\right)$

| | | | | | -z1)/(z11-z1)), i;

$73011|1| 1$ IF $\mathrm{i}=-$-incr then

731 | | | | | | PLOT -0.15,i;-0.15,-i;

$732|1| 1|| \mid$ PLOT $-0.1^{*}\left(1-\left(\right.\right.$ radius $2-\left(\operatorname{SQR}\left(\left((\mathrm{a}-1)^{*} \operatorname{lambda} / 2+\text { radius } 2\right)^{\wedge} 2-\mathrm{j}^{\wedge} 2\right)\right)$ 
||||||||$-z 1) /(z 11-z 1)),-i$

733 | |। |। $\mid$ PLOT $-0.1 *\left(1-\left(\right.\right.$ radius $2-\left(S Q R\left(((a-1) * \text { lambda/2+radius } 2)^{\wedge} 2-\mathrm{j}^{\wedge} 2\right)\right)$

| 11|||| $\mid-z 1) /(z 11-z 1))$, i;

734 । 11|| END IF

735111111 EXIT FOR

736 । 1111 END IF

737 | 1 । 1 NEXT a

738 | | | NEXT $\mathrm{i}$

739 | । END IF

740 | $\mid$ FLOOD flood_cent, 0

741 | 11

742 | । WINDOW \#3

743 | | SET WINDOW 0,incr, 0,1

744 | । SET COLOR 7

745 | | $\mathrm{iii}=0.05$

746 | । FOR $\mathrm{i}=0$ to incr STEP incr/10 !!!

747 | । । FOR $\mathrm{j}=$-iii $/ 2$ to $1+\mathrm{iii} / 2$ STEP $2 *$ iii !

748 । | । PLOT i,j;i,j+iii

749 | । | NEXT j

750 | । NEXT i

751 | | iii=incr $/ 20$

752 I | FOR $\mathrm{i}=0$ to 1.1 STEP 0.1

753 | । | FOR $\mathrm{j}=-\mathrm{iii} / 2$ to incr+iii/2 STEP $2 *$ iii

754 । । । PLOT j,i;j+iii,i

755 | । । NEXT j

756 i I NEXT i

757 | । SET COLOR 15

758 | । PLOT 0,$0 ;$ incr, $0 ;$ incr, $1 ; 0,1 ; 0,0$

$!$

! Make

! grid

! graphs.

759 | । SET TEXT JUSTIFY "center","half"

760111

761 I1। ! For determining the mirror waists of the symmetrical resonator.

762111

763 | I IF confined $\$=" Y "$ then

764 | । I IF $\mathrm{m} 1 \$=" P L A N E "$ OR $\mathrm{m} 2 \$=" P L A N E "$ OR radius $1=$ radius 2 then

765|||| $\mid$ IF radius $1=$ radius 2 then

766 | 1 | | cav_length=cavity_length/2

767 | 1 | | waist=SQR(lambda*cav_length/pi)* $(2 *$ radius $1 \wedge 2 /$ (cavity_length

| $11|1| *($ radius1-cav_length $)))^{\wedge} 0.25$

768 | $1|1| \mathrm{z} 0=\mathrm{SQR}((2 *$ radius1-cavity_length $) *$ cavity_length/4)

769 | | | | w0=waist/SQR $(1+($ cav_length/z0)^2)

770 । $|1| \mathrm{w} 1=0$

771 1 111 END IF

772 | $1 \mid$ IF $m 1 \$=" P L A N E "$ and $m 2 \$=" P L A N E "$ then

773 | || $\mid$ EXIT IF 
774 1 111 ELSE

775 । 1 । 1 IF $\mathrm{m} 2 \$=" S T E P "$ then

776 11 111 IF $\mathrm{m} 1 \$=" P L A N E "$ then

! $\mathrm{w} 1=\mathrm{w} 0$ ELSE $\mathrm{w} 1=\mathrm{waist}$

777 | $11|1|$ cavity_length=cavity_length*2

778 | 1111 | cav_length=cavity_length/2

779 | $\mid$ | $\mid$ | | waist=SQR(lambda*cav_length/pi) $)\left(8 *\right.$ fzt_focus ${ }^{\wedge} 2 /$ (cavity_length

$11111111 *(2 *$ fzt_focus-cav_length $)))^{\wedge} 0.25$

$7801111111 \mathrm{z} 0=\mathrm{SQR}((4 *$ fzt_focus-cavity_length $) *$ cavity_length/4)

781 | $|1||| w 0=$ waist $/ S Q R\left(1+(\text { cav_length } / \mathrm{z} 0)^{\wedge} 2\right)$

782 | $11|1| \mid w 1=w 0$

783 | $11|1|$ cavity_length=cavity_length $/ 2$

784 1 1111 ELSE

785 | |||| $\mid w 1=w a i s t$

786 1 1111 END IF

787 | ||||$j=w 1 /$ step_max_radius

788 | |||| $\mid$ IF $j<1$ then PLOT TEXT, AT incr*j,EXP(-1):"*"

78911111 ELSE

790 1 1111 IF $\mathrm{m} 2 \$=" P L A N E "$ then ! w1=waist ELSE w1=w0

791 | $111 \mid 1$ cavity_length=cavity_length*2

792 | 11111 cav_length=cavity_length/2

793 1 11111 waist=SQR(lambda*cav_length/pi)*(2*radius1^2/(cavity_length

$11111111 *($ radius $1-$ cav_length $)))^{\wedge} 0.25$

$7941111111 \mathrm{z} 0=\mathrm{SQR}((2 *$ radius1-cavity_length $) *$ cavity_length/4)

795 | $1 \mid$ | $\mid$ | w0=waist/SQR $\left(1+(\text { cav_length/z0 })^{\wedge} 2\right)$

796 | $11|1|$ cavity_length=cavity_length $/ 2$

797 | $11|1|$ w1=waist

798 1 1111 ELSE

799 1 11111 w1=w0

800111111 END IF

801 | $|1| \mid$ IF $\mathrm{m} 1 \$=" P L A N E "$ then

802 | | | | | | cavity_length=cavity_length*2

! w l=w0 ELSE w1=waist

803 | |||||| cav_length=cavity_length/2

804 | $|1|||$ waist=SQR(lambda*cav_length/pi $) *(2 *$ radius $2 \wedge 2 /$ (cavity_length

$11111111 *($ radius2-cav_length $)))^{\wedge} 0.25$

$805111111 \mid \mathrm{z} 0=\mathrm{SQR}\left(\left(2^{*} \text { radius2-cavity_length }\right)^{*}\right.$ cavity_length/4)

806 | $11|1|$ w0=waist/SQR $\left(1+(\text { cav_length } / \mathrm{z} 0)^{\wedge} 2\right)$

807 | $|1|||$ cavity_length=cavity_length $/ 2$

808 | |||| $\mid w 1=w 0$

80911111 ELSE

810 | $|1||| w 1=$ waist

$811|1| 1||$ END IF

812||||||$j=w 1 /$ max_radius 1

813 | |||| $\mid \mathrm{IF} j<1$ then PLOT TEXT, AT incr*j,EXP(-1):"*"

814 १॥ | END IF 
815 । $\mid$ | $\mid$ FOR $\mathrm{i}=0$ to incr STEP 2

816 | | | | | | PLOT i,EXP(-(i*dr1/w1)^2);

817 1 1111 PLOT i+1,EXP(-((i+1)*dr1/w1)^2)

818 | $|1|$ NEXT i

819 । |||| PLOT

820 1 $11 \mid$ END IF

821 | | | ELSE

822 | | | | cavity_length_temp=cavity_length-1e-10

823 | $11 \mid \mathrm{z} 0$ _sqrd=cavity_length_temp*(radius1-cavity_length_temp)

824 | | | | z0_sqrd=z0_sqrd*(radius2-cavity_length_temp)

825 | $|1| \mathrm{z} 0$ _sqrd=z0_sqrd*(radius1+radius2-cavity_length_temp)

826 | $111 \mathrm{z0}$ zsqrd=z0_sqrd/(radius2+radius $1-2 *$ cavity_length_temp $)^{\wedge} 2$

827 | । | | z0=SQR(z0_sqrd)

828 | 11 w0 $=S Q R($ lambda*z0/pi)

829|||| $\mathrm{z} \_\mathrm{m} 1=-0.5 *$ radius $1-0.5 * \mathrm{SQR}$ (radius $1^{\wedge} 2-4 * \mathrm{z} 0 \_$sqrd)

830 | $|1| \mathrm{z} \_\mathrm{m} 2=0.5 *$ radius $2+0.5 * \mathrm{SQR}$ (radius $2{ }^{\wedge} 2-4 * \mathrm{z} 0 \_$sqrd)

831|||| $\mathrm{w} 1=\mathrm{w} 0 * \mathrm{SQR}\left(1+\left(\mathrm{z}_{-} \mathrm{m} 1 / \mathrm{z} 0\right)^{\wedge} 2\right)$

832 | । । $\mathrm{w} 2=\mathrm{w} 0 * \mathrm{SQR}\left(1+\left(\mathrm{z} \_\mathrm{m} 2 / \mathrm{z} 0\right)^{\wedge} 2\right)$

833 1 1111

834|||| $\mathrm{j}=w 1 /$ max_radius 1

835 | । | | IF j<1 then PLOT TEXT, AT incr*j,EXP(-1):"**

836 | । | | FOR $\mathrm{i}=0$ to incr STEP 2

837 | । | । | PLOT i,EXP(-(i*dr1/w1)^2);

838 | । | | PLOT $\mathrm{i}+1, \operatorname{EXP}\left(-\left((\mathrm{i}+1)^{*} \mathrm{dr} 1 / \mathrm{w} 1\right)^{\wedge} 2\right)$

839 | || $\mid$ NEXT i

840 | $1 \mid$ PLOT

841 | । | END IF

842 । । END IF

843 | । SET WINDOW $0,1,0,1$

844 I | BOX CLEAR 0.6,1,0.9,1

845 | | BOX LINES 0.6,1,0.9,1

846 I । BOX CLEAR 0.7,0.9,0.8,0.9

847 | । BOX LINES 0.7,0.9,0.8,0.9

848 । । PLOT TEXT, AT 0.8,0.95:"FINAL"

849 I I SET WINDOW 0,incr 1,0,1

850 | । BOX KEEP 0,incr,0,1 IN grid_graph1\$

851 | 11

852 | । WINDOW \#6

853 । I SET WINDOW 0,incr,0,1

854 I I SET COLOR 7

855 | | iii $=0.05$

856 । $\mid$ FOR $\mathrm{i}=0$ to incr STEP incr $/ 10$

857 | । । FOR $\mathrm{j}=$-iii/2 to $1+\mathrm{iii} / 2$ STEP $2 * \mathrm{iii}$

858 |।। | PLOT i,j;i,j+iii
!!!

! Plot gaussian E-Field

! (For non-symmetrical !!! mirrors only).
!! Plot gaussian E-Field

! (For symmetrical and

! half symmetrical

!!! mirrors only). 
859 । । । NEXT j

860 I I NEXT i

861 | । iii $=$ incr $/ 20$

862 I I FOR $\mathrm{i}=0$ to 1.1 STEP 0.1

863 । । । FOR $\mathrm{j}=-\mathrm{iii} / 2$ to incr+iii/2 STEP $2 *$ iii

864 । I । । PLOT j,i;j+iii,i

865 | । I NEXT j

866 | | NEXT i

867 I I SET COLOR 15

868 I। PLOT 0,$0 ;$ incr, $0 ;$ incr, $1 ; 0,1 ; 0,0$

869 I । SET TEXT JUSTIFY "center", "half"

870 । I I

871 | | 1

872 । I I

873 | । IF confined $\$=" Y$ " then

874 | । I IF $\mathrm{m} 1 \$=" P L A N E "$ OR $\mathrm{m} 2 \$==$ PLANE" OR radius1=radius2 then

875 | । । । IF radius1=radius 2 then

876 | | | | | cav_length=cavity_length/2

877 | | | | | waist=SQR(lambda*cav_length/pi)*(2*radius $1{ }^{\wedge} 2 /($ cavity_length

। । | | | *(radius 1 -cav_length $)))^{\wedge} 0.25$

878 | || $\mid \mathrm{z} 0=S Q R((2 *$ radius 1 -cavity_length $) *$ cavity_length/4)

879 | | | | | w0=waist $/ \mathrm{SQR}\left(1+(\text { cav_length/z0 })^{\wedge} 2\right)$

$880 \quad 11|1| \mathrm{w} 2=0$

881 । $\mid$ । END IF

882 | $\mid$ | IF $\mathrm{m} 1 \$=" P L A N E "$ and $\mathrm{m} 2 \$=" P L A N E "$ then

883 । $\mid$ । $\mid$ | EXIT IF

884 | $|1|$ ELSE

885 | | | | | IF $\mathrm{m} 1 \$=" S T E P "$ then

886 । $|1|||$ IF $\mathrm{m} 2 \$=" P L A N E "$ then

887 | |||||| cavity_length=cavity_length*2

888 | | | | | | cav_length=cavity_length $/ 2$

889 | |||||| waist=SQR(lambda*cav_length/pi) $)^{*}\left(8 *\right.$ fzt_focus ${ }^{\wedge} 2 /$ (cavity_length $1111111 \mid *(2 *$ fzt_focus-cav_length $)))^{\wedge} 0.25$

890 | |||||| $\mathrm{z} 0=\mathrm{SQR}((4 *$ fzt_focus-cavity_length $) *$ cavity_length/4)

891 | | | | | | w0=waist/SQR $\left(1+(\text { cav_length } / z 0)^{\wedge} 2\right)$

892 | $11|1| 1 \mathrm{w} 2=w 0$

893 | | | | | | cavity_length=cavity_length/2

894 | $1|1| \mid$ ELSE

895 | | | | | 1 w2=waist

896 | $11|1|$ END IF

897 | $|1|||$ j=w2/step_max_radius

898 । $\mid$ । । । I IF j<1 then PLOT TEXT, AT incr*j,EXP(-1):"**

89911111 ELSE

900 । $\mid$ । | IF $\mathrm{m} 1 \$=" P L A N E "$ then

! w2=w0 ELSE w2=waist

! Make

! grid

!graphs.

!!!

the symmetrical resonator. 
901 | | | | | | cavity_length=cavity_length*2

$902111111 \mid$ cav_length=cavity_length $/ 2$

903 | 11111 | waist=SQR(lambda*cav_length/pi)*(2*radius $2 \wedge 2 /$ (cavity_length

$1111111 \mid *$ (radius2-cav_length) )) $)^{\wedge} 0.25$

$9041111111 \mathrm{zO}=\mathrm{SQR}((2 *$ radius 2 -cavity_length $) *$ cavity_length/4)

$9051111111 \mathrm{w} 0=$ waist $/ \mathrm{SQR}(1+($ cav_length/z0)^2)

906 | | | | | | cavity_length=cavity_length/2

907 1 11111 w2=waist

908 | $1111 \mid$ ELSE

$9091111111 \mathrm{w} 2=\mathrm{w} 0$

$91011111 \mid$ END IF

911 | $|1|||$ IF $2 \$=" P L A N E "$ then

! w2=w0 ELSE w2=waist

912 | $|1|||$ cavity_length=cavity_length*2

913 | | । | | | | cav_length=cavity_length/2

914 | | | | | | | waist=SQR(lambda*cav_length/pi $) *(2 *$ radius $1 \wedge 2 /($ cavity_length

$11111111 *($ radius 1 -cav_length $)))^{\wedge} 0.25$

915 | $11|1| \mathrm{z} 0=\mathrm{SQR}((2 *$ radius 1 -cavity_length $) *$ cavity_length/4)

916 | $11|1|$ w0=waist $/ S Q R(1+($ cav_length/z0)^2)

917 | $\mid$ | | | | cavity_length=cavity_length $/ 2$

918 1111111 w2=w0

919111111 ELSE

92011111|| $\mathrm{w} 2=$ =waist

$92111111 \mid$ END IF

$922|1||| \mid \mathrm{j}=w 2 /$ max_radius2

923 | $11|1|$ IF $j<1$ then PLOT TEXT, AT incr*j,EXP(-1):"*"

$924|1|||$ END IF

925|||| $\mid$ FOR $\mathrm{i}=0$ to incr STEP 2

!!! Plot gaussian E-Field

$92611111 \mid$ PLOT i,EXP(-(i*dr2/w2)^2);

927 | । । । | PLOT $\mathrm{i}+1, \operatorname{EXP}\left(-\left((\mathrm{i}+1)^{*} \mathrm{dr} 2 / \mathrm{w} 2\right)^{\wedge} 2\right)$

928 | | | | NEXT i

! (for symmetrical and

! half symmetrical

92911111 PLOT

!!! mirrors only).

930 । $\mid$ । $\mid$ END IF

931|| $\mid$ ELSE

932 | 1|| $\mathrm{j}=\mathrm{w} 2 /$ max_radius 2

933 | । । I IF j<1 then PLOT TEXT, AT incr*j,EXP(-1):"**

934 | || $\mid$ FOR $i=0$ to incr STEP 2

935 | । । । । PLOT i,EXP(-(i*dr2/w2)^2);

$936|1| 1 \mid$ PLOT $\mathrm{i}+1, \operatorname{EXP}\left(-((\mathrm{i}+1) * \mathrm{dr} 2 / \mathrm{w} 2)^{\wedge} 2\right)$

937 | । । । NEXT i

938 1 $11 \mid$ PLOT

939 । 1 । END IF

940 | । END IF

941111

942 । । SET WINDOW 0,1,0,1 
943 | 1 BOX CLEAR 0.6,1,0.9,1

944 | | BOX LINES 0.6,1,0.9,1

945 | | BOX CLEAR 0.7,0.9,0.8,0.9

946 | | BOX LINES 0.7,0.9,0.8,0.9

947 I | PLOT TEXT, AT 0.8,0.95:"FINAL"

948 I I SET WINDOW 0,incr,0,1

949 | । BOX KEEP 0,incr,0,1 IN grid_graph2\$

950 | | FOR $\mathrm{i}=3$ to 6 STEP 3

951 | | | WINDOW \#i

952 | । I SET WINDOW 0,1,0,1

953 | 11 SET COLOR 15

954 | | | SET TEXT JUSTIFY "center","half"

955 | | | BOX CLEAR 0.4,1,0.9,1

956 | | | BOX LINES 0.4,1,0.9,1

957 | | | PLOT TEXT, AT 0.7,0.95: "HISTORY"

958 | | | BOX CLEAR 0.3,1,0.8,0.9

959 | | | BOX LINES 0.3,1,0.8,0.9

960 । । । PLOT TEXT, AT 0.65,0.85:"EVERY 10"

961 | | | IF i<5 then SET WINDOW 0,max_radius 1,0,1 ELSE SET WINDOW

| | | | 0,max_radius $2,0,1$

962 I 1 NEXT i

963 I I I

964 | | CALL labels

965 I 1 WINDOW \#1

966 I I SET WINDOW 0.05,0.95,0,1

967 I I SET COLOR 15

968 I I SET TEXT JUSTIFY "left","half"

969 | | PLOT TEXT, AT 0.05,0.425:" M1"

970 | I SET TEXT JUSTIFY "right","half"

971 | । PLOT TEXT, AT 0.95,0.425:"M2 "

972 I I SET TEXT JUSTIFY "center","half"

973 I I PLOT TEXT, AT 0.1927,0.425:"Norm Amp vs R"

974 I I PLOT TEXT, AT 0.4880,0.425:"Norm Amp,phase vs R"

975 I I PLOT TEXT, AT 0.8074,0.425:"Norm Amp vs R"

976 I I PLOT TEXT, AT 0.2933,0.80:"1"

977 I I PLOT TEXT, AT 0.2933,0.45:"0"

978 I I PLOT TEXT, AT 0.6947,0.80:"+pi "

979 I I PLOT TEXT, AT 0.6947,0.45+0.035*8:" 0 "

980 I I PLOT TEXT, AT 0.6947,0.45+0.035*6:"-pi "

981 I I PLOT TEXT, AT 0.6947,0.45+0.035*4:"-2pi"

982 I I PLOT TEXT, AT 0.6947,0.45+0.035*2:"-3pi"

983 I I PLOT TEXT, AT 0.6947,0.45:"-4pi"

984 | | ।

985 | । SET CURSOR 2,1

! Header section. 
986 | I PRINT USING "Incr=\#\#; Inct=\#\#":incr,inct

987 | 11

988 I I SET CURSOR 5,1

989 | । IF $\mathrm{m} 1 \$=$ "TIERED" then

990 | | | PRINT "M1=";m1\$;"(";STR\$(tpz1);")"

991 | 1 ELSE

992 | | | PRINT "M1=";m1\$

993 । 1 END IF

994 | 11

995 I I SET CURSOR 5,51

996 I । IF m2\$="TIERED" then

997 I । I PRINT "M2=";m2\$;"(";STR\$(tpz2);")"

998 । 1 ELSE

999 | | | PRINT "M2=";m2\$

1000 | 1 END IF

1001 | । IF $\mathrm{m} 1 \$=$ "STEP" and $\mathrm{m} 2 \$=$ "STEP" then

1002 I I I SET CURSOR 5,51

1003 | | | PRINT "M2=STEP; ALTERNATE=";alternate\$

1004 I 1 END IF

$1005|1|$

1006 | I SET CURSOR 1,51

1007 | | PRINT "R1=";

1008 I I IF m1 $\$=" P L A N E "$ OR m $1 \$=" P A R A B O L I C "$ then

1009 | । | PRINT m1\$[1:5];"; R2=";

$1010 \mid 1$ ELSE

1011|| $\mid$ IF radius $1>0$ and radius $1<1$ then

1012 | | | | PRINT "0";STR\$(radius1);"; R2=";

1013 | | | ELSEIF radius $1>-1$ and radius $1<0$ then

1014 | | | | PRINT "-0";STR\$(-radius1);"; R2=";

1015 | | | ELSE

1016 | | | PRINT STR\$(radius1);"; R2=";

1017 | | | END IF

1018 | | END IF

$1019|1|$

1020 | I IF m2\$="PLANE" OR m2\$="PARABOLIC" then

1021 | | | PRINT m2\$[1:5]

1022 | I ELSE

1023|| $\mid$ IF radius $2>0$ and radius $2<1$ then

1024 | 1 | PRINT "0";STR\$(radius2)

1025|| $\mid$ ELSEIF radius $2>-1$ and radius $2<0$ then

1026 | | | | PRINT "-0";STR\$(-radius2)

1027 | | | ELSE

1028 | | | | PRINT STR\$(radius2)

1029 | | | END IF 
$1030 \mid 1 \mathrm{END}$ IF

1031 | | ।

1032 I I SET CURSOR 2,51

1033 I I PRINT "N1=";

1034 | | n_number $1=$ max_radius $1 \wedge 2 /$ cavity_length/lambda

1035 | | IF n_number $1<1$ then

1036 | | | PRINT "0";STR\$(n_number1)[1:4];"; N2=";

1037 | | ELSE

1038 | | | PRINT STR\$(n_number1)[1:5];"; N2=";

1039 | 1 END IF

$1040|1|$

1041 | $\mid$ n_number2=max_radius $2 \wedge 2 /$ cavity_length/lambda

1042|| IF n_number $2<1$ then

1043 | | | PRINT "0";STR\$(n_number2)[1:4]

1044 | | ELSE

1045 | | | PRINT STR \$(n_number2)[1:5]

1046 | | END IF

1047 | | |

1048 | SET CURSOR 3,1

1049 I I PRINT "W=Wavelength=";STR\$(lambda/le-6);" um"

1050 | | |

1051 | | SET CURSOR 4,51

1052 | । PRINT "Input Wave=";input_wave\$[1:5]

$1053|1|$

1054|| IF $\mathrm{m} 1 \$=$ "STEP" then

1055 | | n_number=step_max_radius^2/cavity_length/lambda

1056 | | I SET CURSOR 4,1

1057 | | | PRINT "Etch Depth=";etch_depth\$

1058 | | | SET CURSOR 3,51

1059 | | | PRINT "\# of Zones=";STR\$(step_tiers);"; $N=" ;$

1060 | $1 \mid$ IF n_number $<1$ then

1061 | | | PRINT "0";STR\$(n_number) [1:4]

1062 | | | ELSE

1063|||| PRINT STR \$(n_number)[1:5]

$1064|1|$ END IF

1065 | | ELSEIF m1 $\$="$ TIERED" and $m 2 \$="$ TIERED" then

1066 | | | SET CURSOR 4,1

1067 | | | PRINT "Etch Depth1,2=W/(";STR \$(2*tpz1);",";STR \$(2*tpz2);")"

1068 | | ELSEIF m1\$="TIERED" then

1069 | | | SET CURSOR 4,1

1070 | | | PRINT "Etch Depth1=";etch_depth1\$

1071 | | ELSEIF m2\$="TIERED" then

1072 | | | SET CURSOR 4,1

1073 | | | PRINT "Etch Depth2=";etch_depth2\$ 
1074 | | END IF

1075 | ।

1076 I $\mid$ SET CURSOR 3,51

1077 | I IF $m 1 \$=" T I E R E D "$ and $m 2 \$=" T I E R E D "$ then

1078 । । । PRINT "tiers1,2=";STR \$(tiers1);",";STR \$(tiers2);"; d=";

1079 | | ELSEIF $m 1 \$=" T I E R E D "$ then

1080 | । | PRINT "tiers1=";STR\$(tiers1);"; $d="$;

1081 | । ELSEIF $\mathrm{m} 2 \$=$ "TIERED" then

1082 | । । PRINT "tiers2=";STR\$(tiers2);"; $d=" ;$

1083 | | ELSE

1084 | | | PRINT "d=";

1085 | । END IF

1086 I I IF cavity_length< 1 then PRINT "0";

1087 I I PRINT STR \$(cavity_length)

1088 | |

1089 | | SET CURSOR 5,24

1090 I I PRINT USING "Begin:\#\#\#\#\#\#\#\#\#/\#\#/\#\#":\&

1091 | 1 \& time $\$$, date $\$[5: 6]$,date $\$[7: 8]$, date $\$[3: 4]$

1092 | | SET CURSOR 1,24

1093 | । PRINT "Prgm = RESONATE ver. 1.0"

1094 I SET CURSOR 4,1

1095 | । IF similate $\$=" Y "$ then PRINT sim\$;";";tol*1e10

1096 11

1097111

! Define the input wave.

1098 11

1099 | । IF input_wave $\$=$ PLANE" then

1100 | | | MAT re2=1

! Initialize all the elements of re $2 \&$ im2.

1101 | | M MAT im2=0

! (re = Real part \& im = Imaginary part)

1102 | | ELSE

1103 | | | cav_length=cavity_length/2

1104 । $\mid$ IF $\mathrm{m} 1 \$<>$ "PLANE" then

$11051111 \mathrm{z} 0=\mathrm{SQR}((2 *$ radius 1 -cavity_length $) *$ cavity_length/4)

1106 | $\mid$ ELSE

1107 1 111 IF m2 $\$<>" P L A N E "$ then $\mathrm{z} 0=\mathrm{SQR}((2 *$ radius2-cavity_length $)$

$11111 *$ cavity_length/4)

1108 | | | END IF

1109 | | | w0=waist/SQR $\left(1+(\text { cav_length/z0 })^{\wedge} 2\right)$

1110 | । 1 eta=ATN(cav_length/z0)

1111 | | Rz=cav_length*(1+(z0/cav_length $\left.)^{\wedge} 2\right)$

1112 | | | FOR $\mathrm{i}=0$ to incr

! Gaussian input wave.

1113 | $\mid$ | re2(i+1) $=2{ }^{*}$ w0/waist ${ }^{*} \operatorname{EXP}\left(-(i * \text { dr } 1 / \text { waist })^{\wedge} 2\right) * \cos ($ eta-k*(cav_length

1114 | $\mid$ | NEXT $\mathrm{i}$
1|| $\left.\left.\mid 1+\left(\mathrm{i}^{*} \mathrm{dr} 1\right)^{\wedge} 2 / 2 / \mathrm{Rz}\right)\right)$
! Real part.

$1115 \|||$ FOR $i=0$ to incr

! Imaginary part. 
1116 | | | im2(i+1)=2*w0/waist*EXP(- $\left.\left(\mathrm{i}^{*} \mathrm{dr} 1 / \mathrm{waist}\right)^{\wedge} 2\right)^{*} \sin (\mathrm{eta}-\mathrm{k} *(\mathrm{cav}$ _length

$\left.\left.|1| 1 \mid+\left(\mathrm{i}^{*} \mathrm{dr} 1\right)^{\wedge} 2 / 2 / \mathrm{Rz}\right)\right)$

1117 | | | NEXT i

! Real part.

1118 | | END IF

$1119|1|$

1120 | 1 max_amp=-1

1121 | | FOR $\mathrm{i}=1$ to incr+1

1122|| $\mid$ amp=SQR(re2(i)^2+im2(i)^2)

1123 | | $\mid \mathrm{zzm}(\mathrm{i})=\mathrm{amp}$

1124 | 1 max_amp=max $\left(\max \_a m p, a m p\right)$

1125 | | NEXT i

1126 I 1 MAT zzm=(1/max_amp $)^{*} z z m$

!!!

1127 | | CALL Simpsons_Rule1(zzm,1,incr+1,initial_int1_amp)

1128 | | initial_int1_amp=initial_int1_amp/incr

1129 | | last_int1_amp=initial_int1_amp

!

1130 | 11

1131 | | FOR $\mathrm{i}=1$ to incr+1

1132|| $\mid \mathrm{j}=(\mathrm{i}-1) * \mathrm{dr} 1$

1133|| $\mid \mathrm{zzm}(\mathrm{i})=\left(\mathrm{re} 2(\mathrm{i})^{\wedge} 2+\mathrm{im} 2(\mathrm{i})^{\wedge} 2\right)^{*} \mathrm{j}$

1134 | | NEXT i

! Find the total

! initial

! amplitude

! distribution

! incident on

! Mirror1.

!

!!!

1135 | | CALL Simpsons_Rule1(zzm,dr1,incr+1,initial_power1)

!!!

! Find the total

! initial power

! incident on

1136 | 1 initial_power1=initial_power $1 * 2 *$ pi

1137 | | last_power1=initial_power1

1138 | | last_power=initial_power1

! mirror1.

1139 | | ।

1140 | | WINDOW \#15

1141 | । SET WINDOW 0.05,0.95,0,1

1142 | | color $=8$

1143 | | phase $=1$

$1144 \mid \mathrm{i} \mathrm{rt}=0$

$1145|1|$

1146 | 1

$1147|1|$

1148 | | FOR transits=1 to max_transits

! $1=$ Reference plane or $0=$ mirror surface.

! Initialize the round trip count.

1149 | | | MAT re1=re2

1150 | | $\mid$ MAT im $1=\mathrm{im} 2$

1151 | | | mirror=1+MOD(transits,2)

! The main body of the prgm begins here.

1152 | | | WINDOW \#1

1153 | | | SET CURSOR 1,1

1154 | | PRINT " ";

1155 | | | SET CURSOR 1,1

1156 | | | SET COLOR 15

1157 | | | IF mirror $=1$ then

1158 | | | | PRINT "(";

! Number of transits or passes.

! $(2$ transits $=1$ round trip $)$.

! Mirror tracker. 
1159 | | | | SET COLOR 11

1160 | $\mid$ | PRINT "<-";

1161 | | | SET COLOR 10

1162 | । | | PRINT "--";

1163 | |। SET COLOR 15

1164 | । | | PRINT USING ") RT= \#\#\# of <\#\#\#":rt,STR\$(max_rt)

1165 । 11 WINDOW \#14

1166 ॥। SET WINDOW 0,incr+3,0,2

1167 । | | SET TEXT JUSTIFY "center","half"

1168 | $\mid 1$ CLEAR

1169 | $\mid$ | SET COLOR 11

1170 । । । MAT phase_delay $=($ phase*k)*zz1

1171 | || ELSEIF mirror=2 then

$1172|1| \mid \mathrm{rt}=\mathrm{rt}+1$

! Round trip count. The initial

1173 | 1 । PRINT "(";

! wave originates from Mirror2.

1174 | | | | SET COLOR 11

1175 | | | | PRINT "--";

1176 | | | SET COLOR 10

1177 | | | PRINT "->";

1178 | | | SET COLOR 15

1179 । 1 । $\mid$ PRINT USING ") RT= \#\# of <\#\#\#":rt-1,STR\$(max_rt)

1180 | || $\mid$ WINDOW \#14

1181 | || SET WINDOW 0,incr +3,0,2

1182 । $\mid$ । SET TEXT JUSTIFY "center","half"

1183 | || CLEAR

1184 | || SET COLOR 10

1185 | | | MAT phase_delay $=\left(\right.$ phase $\left.{ }^{*} \mathrm{k}\right){ }^{*} \mathrm{zz} 2$

1186 | || END IF

1187 | । IF MOD(transits-1,10)=0 then color $=$ color +1

! MOD=remainder.

1188 । $\mid$ IF MOD $($ color, 16$)=0$ then color $=9$

11891111

1190 | । | IF transits $<3$ then

1191 | | | | IF transits $=1$ then

! mirror $=2$

1192 | || $\mid$ FOR $n 2=1$ to incr+1

! Find the resultant $\mathrm{E}$ field

1193 | | | || $\mathrm{r} 2=(\mathrm{n} 2-1) * \mathrm{dr} 2$

! on Mirror2 due to Mirror1.

1194 | $11 \mid 1 \mathrm{r} 2 \mathrm{r} 2=\mathrm{r} 2 * \mathrm{r} 2$

1195 1 $1111 \mathrm{z} 2=$ cavity_length-zz2(n2)

1196111111

1197 | $11 \mid 1$ IF show $\$=" Y "$ then PLOT TEXT, AT n2,1:">"

1198111111

1199 | | | | | FOR $n 1=2$ to incr+1

! Integrate over Mirror1.

1200|||||| $\mid \mathrm{z}, \mathrm{z}_{-} 1(\mathrm{n} 1)=\mathrm{z} 2-\mathrm{zz} 1(\mathrm{n} 1)$

1201|||||| $\mathrm{r} 1=(\mathrm{n} 1-1) * \mathrm{dr} 1$

1202 ||।||| zr1r2,zr1r2_1(n2,n1)=z*z+r1*r1+r2r2 
1203111111 r1r22,r1r22_1(n2,n1)=r1*r $2 * 2$

1204|||||| $\mid r 1$ lambda,r1_lambda_1(n1)=r1/lambda

12051|||||| re1_n1=re1(n1)

1206 |||||| im1_n1=im1(n1)

12071111|| FOR $\mathrm{t} 1=1$ to inct +1

$120811|1| 1 \mid \mathrm{r}=\mathrm{SQR}\left(\mathrm{zr} 1 \mathrm{r} 2-\mathrm{r} 1 \mathrm{r} 22 * \cos \_\mathrm{t}(\mathrm{t} 1)\right)$

$12091111111 \mathrm{kr}=\mathrm{k}^{*} \mathrm{r}$

1210|||||| $\mid \mathrm{h}=(1+\mathrm{z} / \mathrm{r})^{*} \mathrm{r} 1 \_l a m b d a / \mathrm{r}$

1211|||||| $\mid \sin \_k r=\sin (k r)$

$121211|1|||$ cos_kr=cos(kr)

1213|||||| $\mid$ gre $(\mathrm{t} 1)=\mathrm{h} *\left(\mathrm{re} 1 \_\mathrm{n} 1{ }^{*} \sin \_\mathrm{kr}-\mathrm{im} 1 \_\mathrm{n} 1{ }^{*} \cos \_\mathrm{kr}\right)$

$12141|1||| \mid \operatorname{gim}(\mathrm{t} 1)=\mathrm{h} *\left(\mathrm{re} 1 \_\mathrm{n} 1{ }^{*} \cos \_\mathrm{kr}+\mathrm{im} 1 \_\mathrm{n} 1{ }^{*} \sin \_\mathrm{kr}\right)$

1215 | $11|1|$ NEXT $\mathrm{t} 1$

1216 | 11|| CALL Simpsons_Rule2(gre,gim,dt,inct+1,gre2(n1),gim2(n1))

1217 | $|1| \mid$ NEXT $n 1$

1218 || || CALL Simpsons_Rule2(gre2,gim2,dr1,incr+1,re2(n2),im2(n2))

1219 | 1|| NEXT $n 2$

12201|| $\mid$ ELSEIF transits $=2$ then

! Mirror=1

1221|||| $\mid$ FOR $n 1=1$ to incr+1

! Find the resultant $\mathrm{E}$ field

1222||||||$r 1=(\mathrm{n} 1-1) * \mathrm{dr} 1$

! on Mirror1 due to Mirror2.

$1223|1| 1|| \mathrm{r} 1 \mathrm{r} 1=\mathrm{r} 1 *^{\mathrm{r}} \mathrm{r}$

$1224|1| 1|| \mathrm{z} 1=$ cavity_length-zz1(n1)

1225111111

1226 1 1111 IF show $\$=" Y "$ then PLOT TEXT, AT incr+2-n1,1:"<"

12271111111

1228 | $\mid$ | $\mid$ FOR $\mathrm{n} 2=2$ to incr+1

! Integrate over Mirror2.

$1229|1| 1|| \mid$ z,z_2(n2)=z1-zz2(n2)

$12301|1||| \mathrm{r} 2=(\mathrm{n} 2-1) * \mathrm{dr} 2$

$1231|1| 1 \mid 1 \mathrm{zr} 1 \mathrm{r} 2, \mathrm{zr} 1 \mathrm{r} 2 \_2(\mathrm{n} 1, \mathrm{n} 2)=\mathrm{z} * \mathrm{z}+\mathrm{r} 2 * \mathrm{r} 2+\mathrm{r} 1 \mathrm{r} 1$

$1232|1| 1|1| \mathrm{r} 1 \mathrm{r} 22, \mathrm{r} 1 \mathrm{r} 22 \_2(\mathrm{n} 1, \mathrm{n} 2)=\mathrm{r} 1 * \mathrm{r} 2 * 2$

1233111111 r2_lambda,r2_lambda_2(n2)=r2/lambda

$1234|1| 1||$ re1_n2=re1(n2)

1235 | $|1| \mid$ im1_n2=im1(n2)

$12361\|1\| 11$ FOR $2=1$ to inct 1

1237 | $11111 \mathrm{r}=\mathrm{SQR}\left(\mathrm{zr} 1 \mathrm{r} 2-\mathrm{r} 1 \mathrm{r} 22 * \cos _{-} \mathrm{t}(\mathrm{t} 2)\right)$

$12381111111 \mathrm{kr}=\mathrm{k}^{*} \mathrm{r}$

$1239|1| 1|1| 1 \mathrm{~h}=(1+\mathrm{z} / \mathrm{r}) * \mathrm{r} 2 \_l a m b d a / \mathrm{r}$

1240111\|\| $1 \mid \sin \_k r=\sin (k r)$

1241|||||| $\mid \cos \_\mathrm{kr}=\cos (\mathrm{kr})$

1242 | |||| $\mid$ gre $(\mathrm{t} 2)=\mathrm{h} *\left(\mathrm{re} 1 \_\mathrm{n} 2 * \sin \_\mathrm{kr}-\mathrm{im} 1 \_\mathrm{n} 2 * \cos \_\mathrm{kr}\right)$

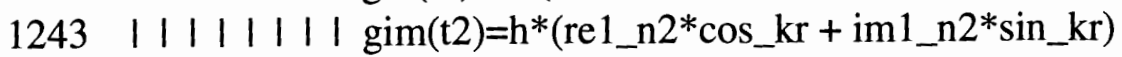

12441111111 NEXT t2

1245 ॥ $|\||$ CALL Simpsons_Rule2(gre,gim,dt,inct+1,gre2(n2),gim2(n2))

1246111111 NEXT n2 
1247 | || $\mid$ CALL Simpsons_Rule2(gre2,gim2,dr2,incr+1,re2(n1),im2(n1))

1248 ||||| NEXT $n 1$

1249 | $\mid$ | $\mathrm{END}$ IF

1250 | $\mid 1$ ELSE

1251 | || IF mirror=2 then

1252 | || $\mid$ FOR $\mathrm{n} 2=1$ to incr+1

! Find the resultant $\mathrm{E}$ field

1253111111

! on Mirror2 due to Mirror1.

12541111111

1255 | $\mid$ । $\mid$ IF show $\$=" Y "$ then PLOT TEXT, AT n2,1:">"

1256111111

1257 | $\mid$ | | | FOR $\mathrm{n} 1=2$ to incr+1

! Integrate over Mirror1.

1258 | $|1|||$ zr1r2=zr1r2_1(n2,n1)

1259 | $|1|||$ r1r22=r1r22_1(n2,n1)

1260|||||| $\mathrm{z}=\mathrm{z}_{-} 1(\mathrm{n} 1)$

1261 | |||| $\mid$ r1_lambda=r1_lambda_1(n1)

1262|||||| $\mid$ re1_n1=re1(n1)

1263 | |||| $\mid \mathrm{im} 1 \_n 1=\operatorname{mim} 1(\mathrm{n} 1)$

1264|||||| $\mid$ FOR $t=1$ to inct +1

$126511111111 \mathrm{r}=\mathrm{SQR}\left(\mathrm{zr} 1 \mathrm{r} 2-\mathrm{r} 1 \mathrm{r} 22 * \cos _{-} \mathrm{t}(\mathrm{t} 1)\right)$

$12661111111 \mathrm{kr}=\mathrm{k}^{*} \mathrm{r}$

1267|||||||| $\mathrm{h}=(1+z / \mathrm{r}) * \mathrm{r} 1 \_l a m b d a / r$

1268 | |||||| $\sin \_k r=\sin (k r)$

1269 | |||||| $\cos \_k r=\cos (k r)$

12701|||||| gre $(\mathrm{t} 1)=\mathrm{h} *\left(\mathrm{re} 1 \_\mathrm{n} 1 * \sin \_\mathrm{kr}-\mathrm{im} 1 \_\mathrm{n} 1 * \cos \_\mathrm{kr}\right)$

$1271|1||||| \operatorname{gim}(\mathrm{t} 1)=h *\left(r e 1 \_n 1 * \cos \_\mathrm{kr}+\mathrm{im} 1 \_n 1 * \sin \_\mathrm{kr}\right)$

1272 | $|1|||$ NEXT t 1

1273|||||| $\mid$ CALL Simpsons_Rule2(gre,gim,dt,inct+1,gre2(n1),gim2(n1))

1274 | $|1| \mid$ NEXT $n 1$

1275 | || $\mid$ CALL Simpsons_Rule2(gre2,gim2,dr1,incr+1,re2(n2),im2(n2))

$1276|1| 1 \mid$ NEXT $n 2$

1277 | $\mid$ | ELSEIF mirror $=1$ then

1278|||| $\mid$ FOR $n 1=1$ to incr+1

12791111111

! Find the resultant $\mathrm{E}$ field

12801111111

! on Mirror1 due to Mirror2.

$1281|1||| \mid$ IF show $\$=" Y "$ then PLOT TEXT, AT incr+2-n1,1:"<"

1282111111

1283 । 11|| FOR n2=2 to incr+1

! Integrate over Mirror2.

1284|||||| $\mid \mathrm{zr} 1 \mathrm{r} 2=\mathrm{zr} 1 \mathrm{r} 2 \_2(\mathrm{n} 1, \mathrm{n} 2)$

1285|||||| $\mid r 1 r 22=r 1 r 22 \_2(n 1, n 2)$

1286|||||| $\mathrm{z}=\mathrm{z} \_2(\mathrm{n} 2)$

1287|||||| $\mid$ r2_lambda=r2_lambda_2(n2)

1288|||||| $\mid r e 1 \_n 2=r e 1(n 2)$

1289 | ||||| im1_n2=im1(n2)

1290|||||| $\mid$ FOR $2=1$ to inct +1 
$12911111111 \mathrm{r}=\mathrm{SQR}\left(\mathrm{zr} 1 \mathrm{r} 2-\mathrm{r} 1 \mathrm{r} 22 * \cos _{-} \mathrm{t}(\mathrm{t} 2)\right)$

$12921111111 \mathrm{kr}=\mathrm{k}^{*} \mathrm{r}$

$1293|1| 1|1| \mathrm{h}=(1+\mathrm{z} / \mathrm{r}) * \mathrm{r} 2$ lambda/r

$129411|1| 1 \mid \sin \_k r=\sin (k r)$

1295|||||||| $\cos \_k r=\cos (k r)$

1296 | 11|||| gre $(\mathrm{t} 2)=\mathrm{h} *\left(\mathrm{re} 1 \_n 2 * \sin \_\mathrm{kr}-\mathrm{im} 1 \_\mathrm{n} 2 * \cos \_\mathrm{kr}\right)$

1297 | $111111 \operatorname{gim}(\mathrm{t} 2)=\mathrm{h} *\left(\mathrm{re} 1 \_\mathrm{n} 2 * \cos \_\mathrm{kr}+\mathrm{im} 1 \_\mathrm{n} 2 * \sin \_\mathrm{kr}\right)$

1298 1 111111 NEXT t2

1299 | |||| $\mid$ CALL Simpsons_Rule2(gre,gim,dt,inct+1,gre2(n2),gim2(n2))

$130011111 \mid$ NEXT n2

1301 | || $\mid$ CALL Simpsons_Rule2(gre2,gim2,dr2,incr+1,re2(n1),im2(n1))

1302 | || $\mid$ NEXT $n 1$

1303 ॥ $1 \mid$ END IF

1304 | | | END IF

13051111

1306 | । | WINDOW \#1

1307 11।

1308 | $\mid$ IF transits=1 then

1309 | || max_amp $=-1$

1310 | || FOR $\mathrm{i}=1$ to incr+1

1311|||| $\mid$ amp=SQR $\left(\mathrm{re} 2(\mathrm{i})^{\wedge} 2+\mathrm{im} 2(\mathrm{i})^{\wedge} 2\right)$

1312 | $|1| \mathrm{zzm}(\mathrm{i})=\operatorname{amp}$

1313 | || $\mid$ max_amp=max (max_amp,amp)

$1314|1|||$ IF max_amp=amp then max_amp_i=i

1315 ॥ $\mid 1$ NEXT $\mathrm{i}$

1316 | || MAT zzm=(1/max_amp $)^{*} z z m$

13171111 CALL Simpsons_Rule1(zzm,1,incr+1,initial_int2_amp)

13181111 initial_int2_amp=initial_int2_amp/incr

1319 | $\mid$ | last_int2_amp=initial_int2_amp

!!!

!

!

! Find the

! total initial

! distribution

! incident on

! mirror2.

13201111

1321 | || ELSEIF mirror $=1$ then

1322 | || max_amp=-1

1323 | | | FOR $\mathrm{i}=1$ to incr+1

$1324|1|||$ amp=SQR(re2(i)^2+im2(i)^2)

$13251111 \mid \mathrm{zzm}(\mathrm{i})=\mathrm{amp}$

1326 11। 1 max_amp=max(max_amp,amp)

1327 | $11 \mid$ IF max_amp=amp then max_amp_i=i

1328 | || NEXT i

1329 | | | MAT zzm=(1/max_amp $)^{*} z z m$

1330 | | | CALL Simpsons_Rule1(zzm,1,incr+1, int1_amp)

1331 | $\mid 1$ int1_amp=int1_amp/incr

!!!

133211111

1333 | | | ELSEIF mirror=2 then

1334 ||। max_amp $=-1$ 
1335 | || FOR $\mathrm{i}=1$ to incr+1

1336|||| $\mid$ amp=SQR(re2(i)^2+im2(i)^2)

$1337 \quad 11111 \mathrm{zzm}(\mathrm{i})=\mathrm{amp}$

1338 | $\mid$ | $\mid$ max_amp=max(max_amp,amp)

1339 | | | | IF max_amp=amp then max_amp_i $i$

1340|||| NEXT i

1341 | || MAT $z z m=\left(1 / \max _{2} a m p\right) * z z m$

1342 | || CALL Simpsons_Rule1(zzm,1,incr+1, int2_amp)

1343 | $|1|$ int2_amp=int2_amp/incr

1344 | $\mid 1$ END IF

13451111

1346 | $\mid$ IF mirror=1 then

$1347 \| 1 \mid$ IF transits $>1$ then last_last_dev_amp=last_dev_amp

1348 | || dev_amp=100*(1-(int1_amp+past_int1_amp)/(2*int1_amp))

1349 | | | | SET CURSOR 3,24

1350 | | | | PRINT "

1351|||| SET CURSOR 3,24

13521111 PRINT USING "Dev Amp =+\#.\#\#\#^^^^\#":dev_amp,"\%"

1353 | $|1|$ past_int1_amp=int1_amp

1354 | | | last_dev_amp=dev_amp

1355|||| IF transits $>3$ and ABS(dev_amp) $<$ dev and ABS(last_dev_amp) $<$ dev and 11111 ABS(last_last_dev_amp) $<$ dev then

1356111111

$1357|1| 1 \mid 1$

1358111111

1359111111

1360|||| $\mid$ WINDOW \#4

1361|||| $\mid$ SET WINDOW 0,1,0,1

1362 । 11 । SET TEXT JUSTIFY "center","half"

$1363\|1\|$ SET COLOR 15

1364 | |||| BOX CLEAR 0.6,1,0.8,0.9

1365 | $\mid$ | | BOX LINES 0.6,1,0.8,0.9

1366 | | | | PLOT TEXT, AT 0.8,0.85:"STABLE"

1367 । $|1| \mid$ WINDOW \#5

1368 | || $\mid$ SET WINDOW 0,1,0,1

1369 । । । । SET TEXT JUSTIFY "center","half"

1370 । $\mid$ | $\mid$ SET COLOR 15

1371 | |||| BOX CLEAR 0.6,1,0.8,0.9

1372 | || $\mid$ BOX LINES 0.6,1,0.8,0.9

1373 | $|1|$ PLOT TEXT, AT 0.8,0.85:"STABLE"

1374 | $|1| \mid$ WINDOW \#14

$137511 \|$ SET WINDOW 0, incr +3,0,2

1376 । । । SET TEXT JUSTIFY "center","half"

1377 |||| CLEAR
$!$

! Find the

! maximum

! amplitude and

! distribution

! incident on

! mirror2.

!!! 
137811111 STOP

1379 | $\mid$ । END IF

1380 | $\mid 1$ END IF

1381 | । । SET CURSOR 4,24

1382 | । । PRINT USING " End:\#\#\#\#\#\# \#\#/\#\#/\#\#":\&

$1383 \quad 11$ \& $\quad$ time $\$$,date $\$[5: 6]$, date $\$[7: 8]$, date $\$[3: 4]$

13841111

13851111

1386 ॥ I IF MOD(transits-1,10) $=0$ then

1387 | $11 \mid$ WINDOW \#6

! Every 10th transit $(1,11,21, \ldots)$.

1388 | || $\mid$ SET COLOR color

1389 | | | | FOR $\mathrm{i}=1$ to incr+1

! mirror $=2$.

1390 | || $\mid$ PLOT (i-1)*dr2,SQR(re2(i)^2+im2(i)^2)/max_amp;

1391 | | | NEXT i

!!! Plot the

! relative

$13921 \|$ PLOT

$1393 \|$ | | IF w $2<>0$ then

1394 1 $11 \|$ SET COLOR 15

1395 | 111 SET TEXT JUSTIFY "center","half"

1396 | $\mid$ | | IF $\mathrm{m} 1 \$=" S T E P "$ then

$1397|1| 1 \mid 1 \mathrm{j}=w 2 /$ step_max_radius2

1398|||||| IF $\mathrm{j}<1$ then PLOT TEXT, AT (incr*j)*dr2,EXP(-1):"**"

1399 १ $|1|$ ELSE

1400||||||$j=w 2 / m a x \_$radius2

$1401|\||||$ IF $j<1$ then PLOT TEXT, AT (incr*j)*dr2,EXP(-1):"*"

1402 | || $\mid$ END IF

1403 | |||| FOR $i=0$ to incr STEP 2

$1404|1| 1||$ PLOT $i * d r 2, \operatorname{EXP}\left(-\left(i^{*} \mathrm{dr} 2 / \mathrm{w} 2\right)^{\wedge} 2\right)$;

1405|||||| PLOT $(i+1)^{*} \operatorname{dr} 2, \operatorname{EXP}\left(-((i+1) * d r 2 / w 2)^{\wedge} 2\right)$

1406 | | | | NEXT $\mathrm{i}$

!!!

!!! amplitudes.

1407 1 $11 \mid$ PLOT

$1408|1|||$ SET COLOR color

1409 | || END IF

1410|| $\mid$ END IF

$1411|1|$

1412 | $\mid$ IF MOD(transits-2,10) $=0$ then

1413 | । । WINDOW \#3

! Plot the theoretical

! gaussian E-Field.

1414|||| SET COLOR color

1415 | | | | FOR $i=1$ to incr+1 !!!

! Every 10th transit $(2,12,22, \ldots)$.

! mirror=1.

$1416|1| 1 \mid$ PLOT $(\mathrm{i}-1)^{*} \mathrm{dr} 1, \mathrm{SQR}\left(\mathrm{re} 2(\mathrm{i})^{\wedge} 2+\mathrm{im} 2(\mathrm{i})^{\wedge} 2\right) / \mathrm{max}_{a} a m p ; !$ relative

1417 | | | | NEXT i

!!! amplitudes.

1418 | || PLOT

1419 | | || IF w $1<>0$ then

1420 | $|1|$ SET COLOR 15

1421 | || | SET TEXT JUSTIFY "center","half" 
1422 1 11 IF $\mathrm{m} 1 \$=" \mathrm{STEP}$ " then

1423||||||$j=w 1 /$ step_max_radius1

$1424|1||| \mid$ IF $j<1$ then PLOT TEXT, AT (incr*j)*dr1,EXP(-1):"*"

$142511 \| 1 \mid$ ELSE

1426||||||$j=w 1 /$ max_radius 1

1427|||||| IF $j<1$ then PLOT TEXT, AT (incr*j)*dr1,EXP(-1):"*"

1428 | | | | END IF

1429|||| $\mid$ FOR $i=0$ to incr STEP 2

$1430\|1\| \| \mid$ PLOT $i * d r 1, \operatorname{EXP}\left(-(i * d r 1 / w 1)^{\wedge} 2\right)$;

1431|||| $\mid$ PLOT $(\mathrm{i}+1)^{*} \operatorname{dr} 1, \operatorname{EXP}\left(-\left((\mathrm{i}+1)^{*} \mathrm{dr} 1 / \mathrm{w} 1\right)^{\wedge} 2\right)$

1432 | 1|| NEXT i

!!!

! Plot the theoretical

! gaussian E-Field.

1433 \|ा | PLOT !!!

$1434|\|| \mid$ SET COLOR color

1435 ॥ । $\mid$ END IF

1436 / $\mid$ END IF

1437 | 11

1438 | $\mid$ । IF mirror=1 then

1439 | || WINDOW \#4

1440 ॥ || SET WINDOW $0,1,0,1$

1441 | | | CLEAR

1442 | || SET COLOR 15

1443 । | | SET TEXT JUSTIFY "center","half"

1444 | । । BOX SHOW grid_graph1\$ AT 0,0 USING "OR"

1445 | | | | PLOT TEXT, AT 0.8,0.85:"M1"

1446 | || SET COLOR 10

! intensified "green"

1447 | || SET WINDOW 0,max_radius1,0,1

1448 | | | FOR $\mathrm{i}=1$ to incr+1

!!! Plot the

1449 | $\mid \|$ PLOT $(\mathrm{i}-1)^{*} \mathrm{dr} 1, \mathrm{SQR}\left(\mathrm{re} 2(\mathrm{i})^{\wedge} 2+\mathrm{im} 2(\mathrm{i})^{\wedge} 2\right) / \mathrm{max} \_a m p ; !$ relative

1450 /1 | NEXT i

!!! amplitudes.

1451 | || PLOT

145211111

145311111

145411111

1455 | 1|| SET WINDOW 0,max_radius 1,-4*pi,pi

1456 1 11 SET COLOR "yellow"

1457 | || IF re2(max_amp_i) $>0$ then

1458 | || jj=ATN(im2(max_amp_i $\left.) / r e 2\left(\max \_a m p \_i\right)+1 e-10\right)$

1459\|\| ELSE

1460 | || $\mid$ IF im2(max_amp_i) $>0$ then

1461|||| $\mid j j=A T N\left(i m 2\left(\max \_a m p \_i\right) / r e 2\left(\max \_a m p \_i\right)+1 e-10\right)+p i$

$146211|1|$ ELSE

1463||||||$j j=A T N\left(i m 2\left(\max \_a m p \_i\right) / r e 2\left(\max \_a m p \_i\right)+1 e-10\right)-p i$

1464111|| END IF

$14651 / 1 \mid$ END IF 
1466 | | | | FOR ii=1 to incr-1 STEP 2

1467 | || $\mid$ FOR $\mathrm{i}=\mathrm{ii}$ to $\mathrm{ii}+1$

1468 | $|1|||$ IF re2(i) $>0$ then

$1469|1||| \mid$ jij=ATN(im2(i)/re2(i)+le-10)

1470111111 ELSE

1471|||||| $\mid$ IF im $2(i)>0$ then

1472|||||| $\mid j j j=A T N(i m 2(i) / r e 2(i)+1 e-10)+p i$

$1473\|1\| 1||$ ELSE

1474|||||| $\mid$ jjj=ATN(im2(i)/re2(i)+le-10)-pi

$147511|1| 1$ END IF

$1476\|1\| 1 \mid$ END IF

1477 | |||| j=jjj-jj-phase_delay(i)+phase_delay(max_amp_i)

$1478|1||| \mid$ IF $i>1$ then

147911|||| $\mid$ DO UNTLL(ABS(last_j-j) $<1.5 *$ pi)

$1480 \|||||||$ IF $j>$ last $j$ then

1481||||||||$j=j-2 *$ pi

$1482111111 \mid$ ELSE

1483||||||||$j=j+2 *$ pi

$1484|1| 1|1| 1$ END IF

$1485|1| 1|1|$ LOOP

$148611111 \mid$ END IF

1487 | |||| last $j=j$

1488 | |||| PLOT (i-1)*dr1,j;

1489 | |||| NEXT $\mathrm{i}$

$1490111 \mid 1$ PLOT

1491 | | | NEXT ii

1492 | $\mid$ | PLOT

1493 | || ELSEIF mirror $=2$ then

1494 । 1 । WINDOW \#5

1495 ॥ । । SET WINDOW 0,1,0,1

1496 ॥ । $\mid$ CLEAR

1497 | || SET COLOR 15

1498 । । । SET TEXT JUSTIFY "center","half"

1499 | $\mid$ | $\mid$ BOX SHOW grid_graph2\$ AT 0,0 USING "OR"

1500 । || PLOT TEXT, AT 0.8,0.85:"M2"

1501|||| SET COLOR 11

! intensified "cyan"

1502 | || $\mid$ SET WINDOW 0,max_radius2,0,1

1503 | $\mid$ | FOR $\mathrm{i}=1$ to incr+1

!!! Plot the

1504 1 $11 \mid$ PLOT $(\mathrm{i}-1)^{*} \mathrm{dr} 2, \mathrm{SQR}\left(\operatorname{re} 2(\mathrm{i})^{\wedge} 2+\mathrm{im} 2(\mathrm{i})^{\wedge} 2\right) / \mathrm{max} \_a m p ; !$ relative

$1505111 \mid$ NEXT $\mathrm{i}$

!!! amplitudes.

1506 | $1 \mid$ PLOT

1507 11111

150811111

! Plot relative phase below.

1509 |||| 
1510 | | | | SET WINDOW 0,max_radius2,-4*pi,pi

1511 | | I SET COLOR "yellow"

1512 | | | | IF re2(max_amp_i) $>0$ then

1513 | | | | | jj=ATN(im2(max_amp_i)/re2(max_amp_i)+1e-10)

1514 | | | | ELSE

1515 | | | | IF im2(max_amp_i) $>0$ then

1516||||||$j j=A T N\left(i m 2\left(m a x \_a m p \_i\right) / r e 2\left(\max \_a m p \_i\right)+1 e-10\right)+p i$

1517 | | | | | ELSE

1518||||||$j j=A T N\left(i m 2\left(m a x \_a m p \_i\right) / r e 2\left(m a x \_a m p \_i\right)+1 e-10\right)-p i$

1519 | | | | | END IF

1520 | | | | END IF

1521 | | | | FOR ii=1 to incr-1 STEP 2

1522 | | | | | FOR i=ii to ii+1

1523|||||| IF re2(i) $>0$ then

1524 | | | | | | jjj=ATN(im2(i)/re2(i)+1e-10)

1525 | | | | | ELSE

1526|||||| $\mid$ IF im2(i) $>0$ then

1527||||||||$j j j=A T N(i m 2(i) / r e 2(i)+1 e-10)+p i$

1528|||||| $\mid$ ELSE

1529|||||||| jjj=ATN(im2(i)/re2(i)+1e-10)-pi

1530 | | | | | | END IF

1531 | | | | | END IF

1532 | | | | | j=jjj-jj-phase_delay(i)+phase_delay(max_amp_i)

1533|||||| IF $i>1$ then

1534|||||| $\mid$ DO UNTIL(ABS(last_j-j)<1.5*pi)

1535|||||||| IF j $>$ last_j then

1536 | | | | | | | j=j-2* pi

1537|||||| $\mid$ ELSE

1538 | | | | | | | j=j+2*pi

1539 | | | | | | | END IF

1540|||||| $\mid$ LOOP

1541 | | | | | END IF

1542 | | | | | last $j=j$

1543 | | | | | | PLOT (i-1)*dr2,j;

1544 | | | | | NEXT i

1545 | | | | | PLOT

1546 | | | | NEXT ii

1547 | | | | PLOT

1548 | | | END IF

1549 | | | |

1550 | | | IF transits $=1$ then

1551 | | | | FOR i=1 to incr+1

1552 | | | | j=(i-1)*dr2

1553|||| $\mid z z m(i)=\left(\operatorname{re} 2(i)^{\wedge} 2+i m 2(i)^{\wedge} 2\right)^{*} j$

!!! 
1554 | | | | NEXT i

! total power

1555 | | | | CALL Simpsons_Rule1(zzm,dr2,incr+1, initial_power2) ! incident

1556 | | | | initial_power2=initial_power $2 * 2 *$ pi

1557 | | | | last_power2=initial_power2

1558 | | | power,power2=initial_power2

! on Mirror2.

1559 | | | |

1560 | | | ELSEIF mirror=1 then

1561 | | | FOR i=1 to incr+1

1562 | | | | j=(i-1)*dr1

1563|||| $\mid z z m(i)=\left(\operatorname{re} 2(i)^{\wedge} 2+i m 2(i)^{\wedge} 2\right)^{* j}$

$$
\text { ! }
$$

!!!

1564 | | | | NEXT i

1565 | | | | CALL Simpsons_Rule1(zzm,dr1,incr+1,power1)

!!!

1566 | | | power $1=$ power $1 * 2 *$ pi

1567 | | | power=power 1

! Find the

! total power

! incident on

! mirror1.

1568 | | | |

1569 | | | ELSEIF mirror=2 then

1570 | | | | FOR $i=1$ to incr+1

$! ! !$

1571 | | | | j=(i-1)*dr2

1572|||| $\mid z z m(i)=\left(\operatorname{re} 2(i)^{\wedge} 2+\mathrm{im} 2(\mathrm{i})^{\wedge} 2\right)^{*} \mathrm{j}$

! Find the

1573 | | | | NEXT i

! total power

! incident on

1574 | | | | CALL Simpsons_Rule1(zzm,dr2,incr+1,power2)

! Mirror2.

1575 | | | power2=power $2 * 2 *$ pi

1576 । । | power=power2

1577 | | | END IF

1578 | | |

1579 | | | |

! NORmalized POWER is the amount remaining of the initial.

1580 | | | |

1581 | | | WINDOW \#1

1582 | I I SET COLOR 15

1583 | | | SET CURSOR 2,24

1584 | | | PRINT "

1585 I I I SET CURSOR 2,24

1586 | | | PRINT USING "Norm Power=+\#.\#\#\#\#^^^^ \#":100*power/initial_power1, 111 | "\%"

$1587|1|$

1588 | | | |

! Plot Power Loss (P.L.) per pass below.

1589 | | | |

1590 | | | IF transits=1 then

! Mirror2 info only.

1591 | | | | loss_per_pass(1)=1-initial_power2/initial_power1

1592 | | | last_power=power

1593 | | | | loss2_per_pass(1)=loss_per_pass (1)

1594 | | | | last_power2=power2

1595 | | | | loss_min,loss_min_old,loss_min1=1e10

1596 | | | | loss_max,loss_max_old,loss_max1=-1e10 
1597 | | | | IF auto_scale $\$=" Y "$ then

1598 | | | | | FOR i=ROUND(LOG10(loss2_per_pass(1)))-1 to 0

1599 | | | | | | IF loss2_per_pass(1) $>10^{\wedge} i$ then

1600 | | | | | | EXIT IF

1601 | | | | | ELSE

1602|||||| $\mid$ loss_max $2=10^{\wedge} i$

1603 | | | | | | loss_min2=10^(i-1)

1604 | | | | | i $=0$

1605|||||| END IF

1606 | | | | | NEXT i

1607 | | | | ELSE

1608 | | | | | loss_min2=0.00001

1609 | | | | loss_max2=1

1610 | | | | END IF

1611 | | | |

1612 | | | ELSEIF mirror=2 then

! Mirror2 info only.

1613 | | | l loss2_per_pass(rt)=1-power2/last_power1

1614 | | | | last_power2=power2

1615 | | | loss_per_pass(transits)=1-power/last_power

1616 | | | last_power=power

1617 | | | | IF auto_scale $\$=" Y "$ then

1618 | | | | FOR i=ROUND(LOG10(loss2_per_pass(rt)))-1 to 0

1619 | | | | | IF loss2_per_pass(rt) $>10^{\wedge} i$ then

1620|||||| $\mid$ EXIT IF

1621 | | | | | ELSE

1622|||||| $\mid$ loss_max $2=10^{\wedge} \mathrm{i}$

1623|||||| $\mid$ loss_min2 $=10^{\wedge}(\mathrm{i}-1)$

1624 | | | | | | i=0

1625|||||| END IF

1626 | | | | NEXT i

1627 | | | | ELSE

1628 | | | | loss_min2=0.00001

1629 | | | | | loss_max2=1

1630 | | | | END IF

1631 | | | |

1632 | | | ELSEIF transits=2 then

1633 | | | loss1_per_pass(1)=1-power1/last_power2

! Mirror1 info only.

1634 | | | | last_power1=power1

1635 | | | | loss_per_pass(transits)=1-power/last_power

1636 | | | last_power=power

1637 | | | | IF auto_scale $\$=" Y "$ then

1638 | | | | FOR i=ROUND(LOG10(loss1_per_pass(1)))-1 to 0

1639 | | | | | IF loss 1_per_pass(1) $>10^{\wedge} i$ then

1640 | | | | | | | EXIT IF 
1641 | | | | | ELSE

1642|||||| $\mid$ loss_max $1=10^{\wedge} \mathrm{i}$

1643 | | | | || loss_min1=10^(i-1)

1644 | | | | | | | i=0

1645 | । | । | END IF

1646 | । | | | NEXT i

1647 | । | | ELSE

1648 | | | | | loss_min $1=0.00001$

1649 | | | | | loss_max $1=1$

1650 । । । | END IF

1651 | | | |

1652 | | | ELSEIF mirror=1 then

1653 | | | | loss1_per_pass(rt)=1-power1/last_power2

! Mirror1 info only.

1654 | | | | last_power l=power1

1655 | । । । loss_per_pass(transits)=1-power/last_power

1656 | | | | last_power=power

1657 | । । I IF auto_scale $\$=" Y "$ then

1658 । । । । | FOR i=ROUND(LOG10(loss1_per_pass(rt)))-1 to 0

1659 | | | | | | IF loss1_per_pass(rt) $>10^{\wedge} i$ then

1660 | | | | | | | EXIT IF

1661|||||| ELSE

1662 | | | | | | loss_max $1=10^{\wedge} \mathrm{i}$

1663 | | | | | | loss_min1=10^(i-1)

1664 | | | | | | | i=0

1665 | । | | | | END IF

1666 | | | | | NEXT i

1667 | | | | ELSE

1668 | | | | | loss_min $1=0.00001$

1669 | | | | | loss_max $1=1$

1670 | | | | END IF

1671 | | | END IF

1672 | | | |

1673 I । I WINDOW \#9

1674 । । I SET COLOR 15

1675 | । | loss_min=min(loss_min,loss_min 1 )

1676 | | | loss_min=min(loss_min,loss_min2)

1677 | | | loss_max=max(loss_max,loss_max 1)

1678 | | | loss_max $=\max ($ loss_max,loss_max2)

1679 I I । I

1680 । । । IF mirror=2 then

1681 | | | | IF loss_min $<>$ loss_min_old OR loss_max<>loss_max_old then

1682 | | | | | CLEAR

1683 | । | | | WINDOW \# 1

1684 | । । | | BOX CLEAR 0.339,0.373,0,0.4 
1685 | । | | | CALL labels

1686 | | | | | WINDOW \#9

1687 | । । | | SET WINDOW 0,max_transits,LOG10(loss_min),LOG10(loss_max)

| । | | | | +(LOG10(loss_max)-LOG10(loss_min))/7

1688 | | | | | SET COLOR 7

1689 | | | | | FOR i=0 to max_transits STEP $\max (1, \mathrm{INT}($ max_transits/10))

1690 | । | | | | PLOT i,LOG10(loss_min);i,LOG10(loss_max)

1691 | । I | | NEXT i

1692 । । । । । SET TEXT JUSTIFY "right","half"

1693 | । | | | FOR i=ROUND(LOG10(loss_min)) to ROUND(LOG10(loss_max))

1694 | । | | | | SET COLOR 7

1695 | | | | | | PLOT 0,i;max_transits,i

1696 | | | | | | SET COLOR 15

1697 | । । | | PLOT TEXT, AT -max_transits/40,i:STR\$(i+2)

1698 | । । | | NEXT i

1699 । । । । । SET TEXT JUSTIFY "center","top"

1700 | । | | | PLOT TEXT, AT max_transits/2,LOG10(loss_max)

| । । । । | +(LOG10(loss_max)-LOG10(loss_min))/7:"P.L./Pass/Mirror"

1701 I । । । । SET TEXT JUSTIFY "right","half"

1702 | । । । | PLOT 0,LOG10(loss_min);0,LOG10(loss_max);

1703 | । । । | PLOT max_transits,LOG10(loss_max);max_transits,LOG10(loss_min);

1704 | । I | | PLOT 0,LOG10(loss_min)

1705 | । | | | IF loss_min $<0$ and loss_max $>0$ then PLOT 0,0;max_transits, 0

1706 । । । । । SET COLOR 12

1707 | | | | | FOR $\mathrm{i}=1$ to transits

1708 । । । । | । PLOT i,LOG10(loss_per_pass(i));

1709 | । । | | NEXT i

1710 । $\mid$ । | P PLOT

1711 | । । । | IF transits $>1$ then

1712 | | | | | | SET COLOR 10

! M1.

1713 | । । । | | FOR i=2 to $2 *$ rt-2 STEP 2

1714 | | । | | | | PLOT i,LOG10(loss1_per_pass(i/2));

1715 | | | | | NEXT $\mathrm{i}$

1716 । । । । END IF

1717 | |। | PLOT

1718 | |। | | SET COLOR 11

! M2.

1719 | । । । | FOR $\mathrm{i}=1$ to $2 *$ rt- 1 STEP 2

1720 | । | | | | PLOT i,LOG10(loss2_per_pass((i+1)/2));

1721 | | | | | NEXT i

1722 । $\mid$ । | $\mid$ PLOT

1723 | | | | ELSE

1724 । । । । । SET TEXT JUSTIFY "center","top"

1725 । | । | | BOX CLEAR 0,max_transits,LOG10(loss_max)+(LOG10(loss_max)

I I I I | | -LOG10(loss_min))/15,LOG10(loss_max)+(LOG10(loss_max) 
| । | |। | -LOG10(loss_min))/5

1726 । । । । PLOT TEXT, AT max_transits/2,LOG10(loss_max)

। । । । । । +(LOG10(loss_max)-LOG10(loss_min))/7:"P.L./Pass/Mirror"

1727 । $\mid$ । । । SET COLOR 12

1728 । । । । । PLOT transits-1,LOG10(loss_per_pass(transits-1));

1729 | । । । । PLOT transits,LOG10(loss_per_pass(transits))

1730 । । । । | SET COLOR 11

1731 | । । । | PLOT 2*rt-3,LOG10(loss2_per_pass(rt-1));

1732 । । । । । PLOT 2*rt-1,LOG10(loss2_per_pass(rt))

1733 । $\mid$ । । END IF

1734 | । । | WINDOW \#15

1735 । । । । SET TEXT JUSTIFY "center","half"

1736 । । । । BOX CLEAR 0.63,0.95,0,1

1737 I I I I SET COLOR 11

1738 । । । । PLOT TEXT, AT 0.776,0.5:"M2

1739 | । । I SET COLOR 15

1740 | । | । PLOT TEXT, AT 0.776,0.5:\&

1741 | । । । \&USING\$(" P.L.:\#\#.\#\#^^^\#",100*loss2_per_pass(rt),"\%")

1742 । । । I SET COLOR 15

1743 । । । । PLOT TEXT, AT 0.224,0.5:"M1

1744 । । । । BOX CLEAR 0.35,0.63,0,1

1745 । । । । PLOT TEXT, AT 0.488,0.5:\&

1746 । । । । \& USING\$("Final P.L.:+\#.\#\#^^^ \#",100*loss_per_pass(transits),"\%")

1747 | | | |

1748 | | | ELSEIF mirror=1 then

1749 | । | | IF loss_min $<>$ loss_min_old OR loss_max $<>$ loss_max_old then

1750 । । । | C CLEAR

1751 | । । । | WINDOW \#1

1752 । । । । । BOX CLEAR 0.339,0.373,0,0.4

1753 | | | | | CALL labels

1754 | । | | | WINDOW \#9

1755 । । । । । SET WINDOW 0,max_transits,LOG10(loss_min),LOG10(loss_max)

| । । | | | +(LOG10(loss_max)-LOG10(loss_min))/7

1756 । $\mid$ । । | SET COLOR 7

1757 | । | | | FOR i=0 to max_transits STEP $\max (1$, INT(max_transits/10))

1758 । । । । । । PLOT i,LOG10(loss_min);i,LOG10(loss_max)

1759 | । | | | NEXT i

1760 । । । । । SET TEXT JUSTIFY "right","half"

1761 | । | | | FOR i=ROUND(LOG10(loss_min)) to ROUND(LOG10(loss_max))

1762 । |||| $\mid$ SET COLOR 7

1763 | । | | | | PLOT 0,i;max_transits,i

1764 । $\mid$ । । । SET COLOR 15

1765 । । । । । । PLOT TEXT, AT -max_transits/40,i:STR\$(i+2)

1766 | | | | | NEXT i 
1767 । । । । । SET TEXT JUSTIFY "center","top"

1768 | । । । | PLOT TEXT, AT max_transits/2,LOG10(loss_max)

| । । | । | +(LOG10(loss_max)-LOG10(loss_min))/7:"P.L./Pass/Mirror"

1769 । । । । । SET TEXT JUSTIFY "right","half"

1770 | | | | | PLOT 0,LOG10(loss_min);0,LOG10(loss_max);

1771 । | । । । PLOT max_transits,LOG10(loss_max);max_transits,LOG10(loss_min);

1772 । । । । | PLOT 0,LOG10(loss_min)

1773 | । | | | IF loss_min<0 and loss_max >0 then PLOT 0,0;max_transits, 0

1774 I I I I I SET COLOR 12

1775 | | | | | FOR $\mathrm{i}=1$ to transits

1776 | । I | | | PLOT i,LOG10(loss_per_pass(i));

1777 | । । । | NEXT i

1778 | । | | PLOT

1779 । । । । । SET COLOR 10

! M1

1780 | । । । | FOR i=2 to $2 *$ rt STEP 2

1781 | । | । | | PLOT i,LOG10(loss1_per_pass(i/2));

1782 | । | । | NEXT i

1783 | | | | | PLOT

1784 । । । । | SET COLOR 11

! M2

1785 | । । । | FOR $\mathrm{i}=1$ to $2 *$ rt-1 STEP 2

1786 । | । | | | PLOT i,LOG10(loss2_per_pass((i+1)/2));

1787 | । I I | NEXT i

1788 I । । । PLOT

1789 | । । । ELSE

1790 । । । । । SET TEXT JUSTIFY "center","top"

1791 | | | | | BOX CLEAR 0,max_transits,LOG10(loss_max)+(LOG10(loss_max)

| | | | | | -LOG10(loss_min))/15,LOG10(loss_max)+(LOG10(loss_max)

I | । I । I -LOG10(loss_min))/5

1792 | । । । | PLOT TEXT, AT max_transits/2,LOG10(loss_max)

। । । । । +(LOG10(loss_max)-LOG10(loss_min))/7:"P.L./Pass/Mirror"

1793 । । । । I SET COLOR 12

1794 । । । । । PLOT transits-1,LOG10(loss_per_pass(transits-1));

1795 I I I I I PLOT transits,LOG10(loss_per_pass(transits))

1796 I । । । I SET COLOR 10

1797 । । । । । IF transits=2 then

1798 । । । । । । PLOT 2*rt,LOG10(loss1_per_pass(rt))

1799 | । | | | ELSE

1800 | । । । । । PLOT 2*rt-2,LOG10(loss1_per_pass(rt-1));

1801 | । | | | | PLOT 2*rt,LOG10(loss1_per_pass(rt))

1802 | । | | | END IF

1803 । । । I END IF

1804 । । । | WINDOW \#15

1805 । । । । SET TEXT JUSTIFY "center","half"

1806 । । । | BOX CLEAR 0,0.35,0,1 
1807 I । । । SET COLOR 10

1808 । | | | PLOT TEXT, AT 0.224,0.5:"M1 "

1809 । । । | SET COLOR 15

1810 । । । । PLOT TEXT, AT 0.224,0.5:\&

1811 । । । । \& USING\$(" P.L.:+\#.\#\#^^^\#",100*loss1_per_pass(rt),"\%")

1812 | । | | SET COLOR 15

1813 । । । । PLOT TEXT, AT 0.776,0.5:"M2

1814 । । । | BOX CLEAR 0.35,0.63,0,1

1815 । । | । PLOT TEXT, AT 0.488,0.5:\&

1816 । । । । \& USING\$("Final P.L.:+\#.\#\#^^^ \#",100*loss_per_pass(transits),"\%")

1817 | | | END IF

1818 | । | loss_min_old=loss_min

1819 | | | loss_max_old=loss_max

1820 | | । ।

1821 I। ।

! Plot Mirror2's amplitude variation per Round Trip(RT) below.

1822 | | | ।

1823 | । | IF transits=3 then

! Mirror=2.

1824 । । । । intvar2_per_pass(1),intvar2_max,intvar2_min=int2_amp/last_int2_amp-1

1825 | | | | ।

1826 | । | ELSEIF mirror=2 and transits $>3$ then

! Mirror2 info only.

1827 I । I । WINDOW \#10

1828 I । I I SET COLOR 15

1829 । । । | intvar2_per_pass(rt-1)=int2_amp/last_int2_amp-1

1830 | । । । intvar2_min=min(intvar2_min,intvar2_per_pass(rt-1))

1831 | । । | intvar_min=min(intvar_min,intvar1_min)

1832 । । । I intvar_min=min(intvar_min,intvar2_min)

1833 | । । । intvar2_max=max(intvar2_max,intvar2_per_pass(rt-1))

1834 | | । | intvar_max=max(intvar_max,intvar1_max)

1835 | । । । intvar_max=max(intvar_max,intvar2_max)

1836 | । | | IF intvar_min<>intvar2_min_old OR intvar_max<>intvar2_max_old then

1837 । । | । CLEAR

1838 | । | । | WINDOW \#1

1839 । । । । | BOX CLEAR 0.603,0.661,0,0.4

1840 । । । | | CALL labels

1841 । । । । | WINDOW \#10

1842 । । । । । SET WINDOW 0,max_rt,intvar_min-(intvar_max-intvar_min)/25,

I । I । । | intvar_max+(intvar_max-intvar_min)/7

1843 । । । । | SET COLOR 7

1844 | । | । | FOR i=0 to max_rt STEP $\max \left(1, \mathrm{INT}\left(\max \_r t / 10\right)\right)$

1845 | | | । | | PLOT i,intvar_min;i,intvar_max

1846 । । | | NEXT i

1847 । । । । । SET COLOR 15

1848 । | | | | BOX CLEAR 0,max_rt,intvar_max+(intvar_max-intvar_min)/15,

। । । । | । intvar_max+(intvar_max-intvar_min)/5 
1849 । । । । । SET TEXT JUSTIFY "center","top"

1850 | । । । PLOT TEXT, AT max_rt/2,intvar_max+(intvar_max-intvar_min)/7:\&

1851 । । । | \& USING\$("Decay/RT:+\#.\#^^^",100*intvar2_per_pass(rt-1))

1852 । । । । । SET TEXT JUSTIFY "right","half"

1853 | | | | | i=-max_rt/40

1854 | । । । । PLOT TEXT, AT i,intvar_max:STR\$(ROUND(100*intvar_max))

1855 । । । । । PLOT TEXT, AT i,intvar_min:STR\$(ROUND(100*intvar_min))

1856 | | | | | PLOT TEXT, AT i,intvar_min+(intvar_max-intvar_min)/2:"\% "

1857 । । । । । PLOT 0,intvar_min;0,intvar_max;

1858 | । | | | PLOT max_rt,intvar_max;max_rt,intvar_min;0,intvar_min

1859 | । । | । IF intvar_min $<0$ and intvar_max $>0$ then

1860 । $\mid$ । । । SET COLOR 7

1861 । | | | । | PLOT 0,0;max_rt,0

1862 । । । | | END IF

1863 । । । । I SET COLOR 11

1864 । । । | | FOR $\mathrm{i}=1$ to $\mathrm{rt}-1$

1865 | । | । | | PLOT i,intvar2_per_pass(i);

1866 | । I | | NEXT i

1867 | | | | | PLOT

1868 | । । | ELSE

1869 । । । । । SET TEXT JUSTIFY "center","top"

1870 । । । । । BOX CLEAR 0.57*max_rt,max_rt,intvar_max+(intvar_max-

I । | | | intvar_min)/15,intvar_max+(intvar_max-intvar_min) $/ 5$

1871 | । । | | PLOT TEXT, AT max_rt/2,intvar_max+(intvar_max-intvar_min)/7:\&

1872 । । । । । \& USING\$("Decay/RT:+\#.\#^^^",100*intvar2_per_pass(rt-1))

1873 । |||| SET COLOR 11

1874 | । | । | PLOT rt-2,intvar2_per_pass(rt-2);

1875 । । । । । PLOT rt-1,intvar2_per_pass(rt-1)

1876 । । । | END IF

1877 | | | | last_int2_amp=int2_amp

1878 I । । । intvar_max_old,intvar2_max_old=intvar_max

1879 । । । । intvar_min_old,intvar2_min_old=intvar_min

1880 I | । I ।

1881 | । । । ! Plot Mirror1's amplitude variation per Round Trip(RT) below.

1882 || | |

1883 | $\mid$ | ELSEIF transits=2 then ! Mirror=1.

1884 | । | | intvar1_per_pass(1),intvar1_max,intvar1_min=int1_amp/last_int1_amp-1

1885 | | | | intvar1_min_old,intvar2_min_old=1e10

1886 | | | | intvar1_max_old,intvar2_max_old=-1e10

1887 | । I। ।

1888 । । । ELSEIF mirror=1 then

1889 । । । । WINDOW \#8

1890 । । I । SET COLOR 15

1891 | । I | intvar1_per_pass(rt)=int1_amp/last_int1_amp-1 


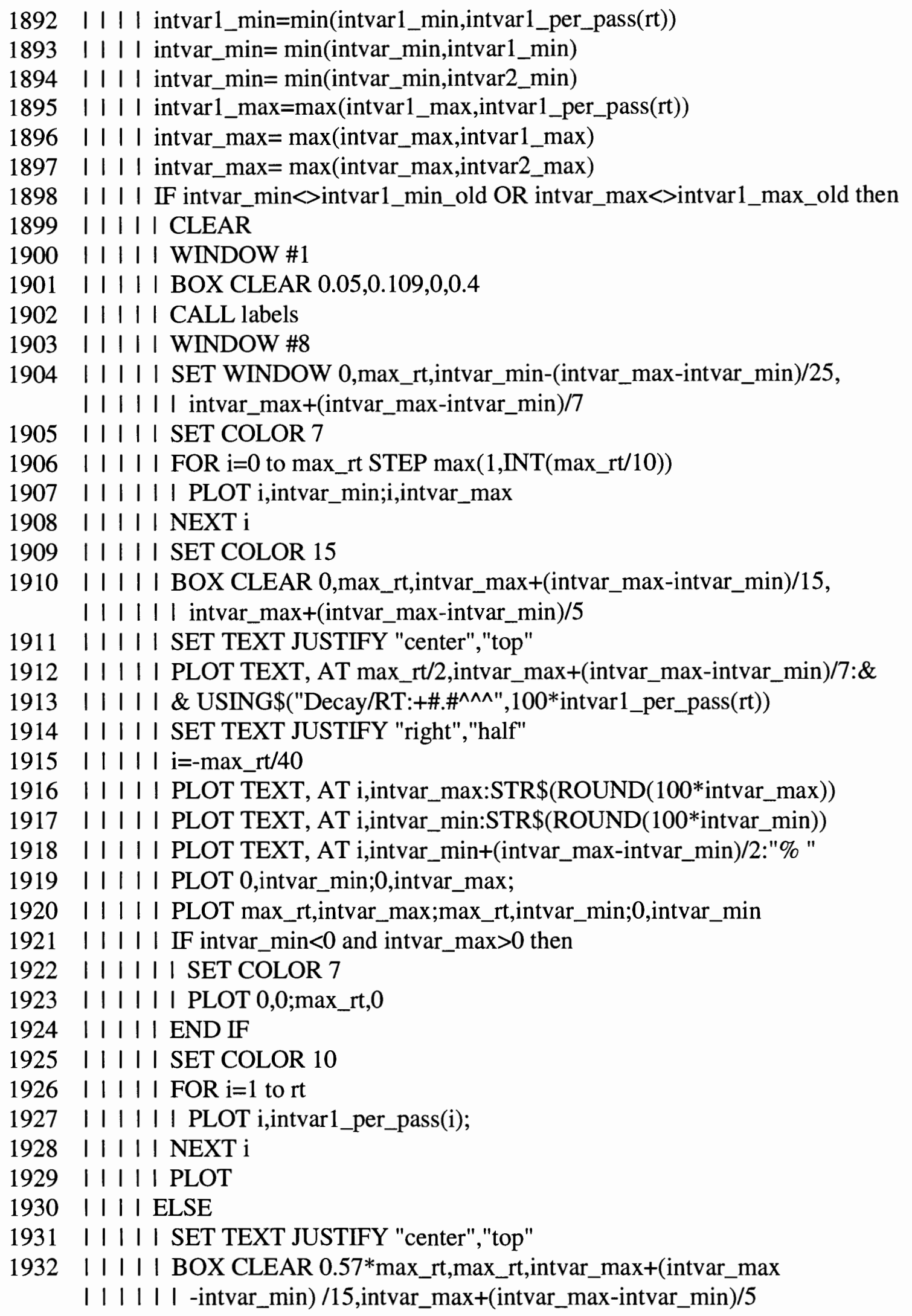


1933 | | | | | PLOT TEXT, AT max_rt/2,intvar_max+(intvar_max-intvar_min)/7:\&

1934 । । । । । \& USING\$("Decay/RT:+\#.\#^^^",100*intvar1_per_pass(rt))

1935 । । । । | SET COLOR 10

1936 | । । | | PLOT rt-1,intvar1_per_pass(rt-1);

1937 | । I | | PLOT rt,intvar1_per_pass(rt)

1938 । । । | END IF

1939 | | | | last_int1_amp=int1_amp

1940 | | । | intvar_max_old,intvar1_max_old=intvar_max

1941 | । | | intvar_min_old,intvar1_min_old=intvar_min

1942 | | | END IF

1943 | || ।

1944 I I NEXT transits

! End of main loop.

1945 | | ।

1946 | । ।

! Halt the program for a full screen view.

1947 | | |

1948 | । transits=transits-1

1949 | । WINDOW \#14

1950 | । SET WINDOW 0,incr+3,0,2

1951 I । SET TEXT JUSTIFY "center","half"

1952 I | CLEAR

1953 I । GET KEY key

1954 | । FOR $\mathrm{i}=8$ to 15

1955 । । | CLOSE \# $\mathrm{i}$

1956 I | NEXT i

1957 | । ।

1958 । ।

1959 | । ।

! Halt the program for a smaller screen view

1960 | । ।

1961 | | OPEN \#8 : SCREEN 0.110,0.338,0.19,0.4

1962 | । OPEN \#9 : SCREEN 0.374,0.602,0.19,0.4

1963 | । OPEN \#10: SCREEN 0.662,0.890,0.19,0.4

1964 I । OPEN \#11: SCREEN 0.110,0.338,0.15,0.18

1965 | । OPEN \#12: SCREEN 0.374,0.602,0.15,0.18

1966 | । OPEN \#13: SCREEN 0.662,0.890,0.15,0.18

1967 | । OPEN \#14: SCREEN 0.090,0.935,0.815,0.835

1968 | । OPEN \#15: SCREEN 0.05,0.95,0.11,0.15

1969 | । WINDOW \#15

1970 I I SET WINDOW 0.05,0.95,0,1

1971 । । WINDOW \#1

1972 । । BOX CLEAR 0,1,0,0.4

1973 | | CALL labels

1974 । । ।

1975 | । ।

! Plot Power Loss (P.L.) per pass below.

1976 । । ।

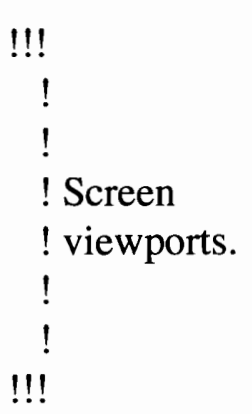


1977 | | WINDOW \#9

1978 | I SET COLOR 7

1979 I । SET WINDOW 0,max_transits,LOG10(loss_min)-(LOG10(loss_max)

| | | | -LOG10(loss_min))/25,LOG10(loss_max)+(LOG10(loss_max)

| | | | -LOG10(loss_min))/5

1980 | । FOR $\mathrm{i}=0$ to max_transits STEP $\max (1, \mathrm{INT}($ max_transits/10))

1981 | । | PLOT i,LOG10(loss_min);i,LOG10(loss_max)

1982 | | NEXT i

1983 । । SET TEXT JUSTIFY "right","half"

1984 | | FOR i=ROUND(LOG10(loss_min)) to ROUND(LOG10(loss_max))

1985 I I | SET COLOR 7

1986 I । I PLOT 0,i;max_transits,i

1987 | | | SET COLOR 15

1988 | । | PLOT TEXT, AT -max_transits/40,i:STR \$(i+2)

1989 | | NEXT i

1990 I I SET TEXT JUSTIFY "center","top"

1991 I I PLOT TEXT, AT max_transits/2,LOG10(loss_max)+(LOG10(loss_max)

I । I -LOG10(loss_min))/5:"P.L./Pass/Mirror"

1992 I I SET TEXT JUSTIFY "right","half"

1993 I | PLOT 0,LOG10(loss_min);0,LOG10(loss_max);

1994 I I PLOT max_transits,LOG10(loss_max);max_transits,LOG10(loss_min);

1995 I I PLOT 0,LOG10(loss_min)

1996 | I IF loss_min<0 and loss_max $>0$ then

1997 I । I SET COLOR 7

1998 | | | PLOT 0,0;max_transits,0

1999 | | END IF

2000 | I SET COLOR 12

2001 | | FOR $\mathrm{i}=1$ to transits

2002 I I I PLOT i,LOG10(loss_per_pass(i));

2003 | I NEXT $\mathrm{i}$

2004 | । PLOT

2005 | | SET COLOR 10

!M1

2006 | । FOR $\mathrm{i}=2$ to $2 *$ rt STEP 2

2007 | । | PLOT i,LOG10(loss1_per_pass(i/2));

2008 | | NEXT i

2009 | । PLOT

2010 | | SET COLOR 11

! M2

2011 | | FOR i=1 to $2 *$ rt-1 STEP 2

2012 | । । PLOT i,LOG10(loss2_per_pass((i+1)/2));

2013 | | NEXT i

2014 | । PLOT

2015 | | WINDOW \#15

2016 । I SET TEXT JUSTIFY "center","half"

2017 | | SET COLOR 15 
2018 | | PLOT TEXT, AT 0.224,0.5:\&

2019 | । \& USING\$("M1 P.L.:+\#.\#\#^^^ \#",100*loss1_per_pass(rt),"\%")

2020 | । SET COLOR 15

2021 | । PLOT TEXT, AT 0.776,0.5:\&

2022 । । \& USING\$("M2 P.L.:+\#.\#\#^^^ \#",100*loss2_per_pass(rt-1),"\%")

2023 | । PLOT TEXT, AT 0.488,0.5:\&

2024 । । \& USING\$("Final P.L.:+\#.\#\#^^^\#",100*loss_per_pass(transits),"\%")

2025111

2026111

! Plot Mirror1's amplitude variation per Round Trip(RT) below.

2027 | I ।

2028 । I WINDOW \#8

2029 | I SET COLOR 7

2030 I I SET WINDOW 0,max_rt,intvar_min-(intvar_max-intvar_min)/25,

I I I intvar_max+(intvar_max-intvar_min)/5

2031 | | FOR $\mathrm{i}=0$ to $\max \_$rt $S T E P \max \left(1, \mathrm{INT}\left(\max \_\mathrm{rt} / 10\right)\right)$

2032 | | | PLOT i,intvar_min;i,intvar_max

2033 | I NEXT i

2034 | I SET COLOR 15

2035 । I SET TEXT JUSTIFY "center","top"

2036 I । PLOT TEXT, AT max_rt/2,intvar_max+(intvar_max-intvar_min)/5:\&

2037 I I \& USING\$("Decay/RT:+\#.\#^^^",100*intvar1_per_pass(rt))

2038 I I SET TEXT JUSTIFY "right","half"

2039 | । i $=-$ max_rt/40

2040 I I PLOT TEXT, AT i,intvar_max:STR \$(ROUND(100*intvar_max))

2041 I । PLOT TEXT, AT i,intvar_min:STR\$(ROUND(100*intvar_min))

2042 I । PLOT TEXT, AT i,intvar_min+(intvar_max-intvar_min)/2:"\% "

2043 I I PLOT 0,intvar_min;0,intvar_max;

2044 I I PLOT max_rt,intvar_max;max_rt,intvar_min;0,intvar_min

2045 | । IF intvar_min $<0$ and intvar_max $>0$ then

2046 I I I SET COLOR 7

2047 I | | PLOT 0,0;max_rt,0

2048 | । END IF

2049 I I SET COLOR 10

2050 | । FOR $\mathrm{i}=1$ to $\mathrm{rt}$

2051 I I । PLOT i,intvar1_per_pass(i);

2052 | | NEXT i

2053 I । PLOT

2054 I I

2055 1 11

2056 11

2057 I । WINDOW \#10

2058 | । SET COLOR 7

2059 I I SET WINDOW 0,max_rt,intvar_min-(intvar_max-intvar_min)/25,

$1 \mid$ intvar_max+(intvar_max-intvar_min)/5 
2060 | | FOR i=0 to max_rt STEP $\max \left(1, \mathrm{INT}\left(\max \_r t / 10\right)\right)$

2061 | | | PLOT i,intvar_min;i,intvar_max

2062 | | NEXT i

2063 I I SET COLOR 15

2064 I I SET TEXT JUSTIFY "center","top"

2065 | । PLOT TEXT, AT max_rt/2, intvar_max+(intvar_max-intvar_min)/5:\&

2066 I I \& USING\$("Decay/RT:+\#.\#^^^",100*intvar2_per_pass(rt-1))

2067 I I SET TEXT JUSTIFY "right","half"

2068 | | i =-max_rt/40

2069 | । PLOT TEXT, AT i,intvar_max:STR $\$(\operatorname{ROUND}(100 *$ intvar_max $))$

2070 I I PLOT TEXT, AT i,intvar_min:STR \$(ROUND(100*intvar_min))

2071 | | PLOT TEXT, AT i,intvar_min+(intvar_max-intvar_min)/2:"\% "

2072 | I PLOT 0,intvar_min;0,intvar_max;

2073 | | PLOT max_rt,intvar_max;max_rt,intvar_min;0,intvar_min

2074 | | IF intvar_min $<0$ and intvar_max $>0$ then

2075 । | | SET COLOR 7

2076 | | | PLOT 0,0;max_rt,0

2077 | । END IF

2078 I I SET COLOR 11

2079 | | FOR $\mathrm{i}=1$ to rt-1

2080 | | | PLOT i,intvar2_per_pass(i);

2081 | | NEXT $\mathrm{i}$

2082 | | PLOT

2083 | । CLOSE \#14

2084 | । OPEN \#14: SCREEN 0.04,0.95,0.815,0.835

2085 | | WINDOW \#14

2086 । I SET COLOR 15

2087 | I SET WINDOW 0.04,0.95,0,2

2088 I I SET TEXT JUSTIFY "left","half"

2089 | । diameter $\$=$ USING $\$(" M 1$ Radius=\#.\#\#^^^ mm",max_radius1/1e-3)

2090 | | PLOT TEXT, AT 0.0478,1:diameter\$

2091 | | diameter $\$=U S I N G \$(" M 2$ Radius=\#.\#\#^^^ mm",max_radius2/le-3)

2092 I | PLOT TEXT, AT 0.6732,1:diameter\$

2093 | I SET TEXT JUSTIFY "center","half"

2094 I । PLOT TEXT, AT 0.4856,1:resonator\$

2095 | । GET KEY key

2096 I LOOP

2097 | ।

2098 END SUB

$2099 \quad 1$

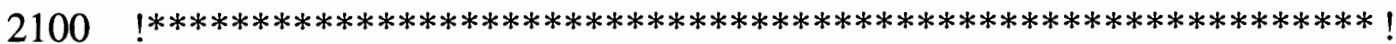

2101

2102

SUBROUTINE LABELS: Labels the $x$-axis.

$2103 \quad$ 
2104 SUB labels

2105 | WINDOW \#11

2106 I SET WINDOW 0,max_rt,0,1

2107 I SET TEXT JUSTIFY "center","bottom"

2108 I SET COLOR 15

2109 I PLOT TEXT, AT 0,0:"0"

2110 I PLOT TEXT, AT max_rt/2,0:STR $\$\left(\max \_r t / 2\right)$

2111 I PLOT TEXT, AT max_rt,0:STR $\$$ (max_rt)

2112 I WINDOW \#12

2113 | SET WINDOW 0,max_transits, 0,1

2114 I SET TEXT JUSTIFY "center","bottom"

2115 I SET COLOR 15

2116 I PLOT TEXT, AT 0,0:"0"

2117 I PLOT TEXT, AT max_transits/2,0:STR $\$($ max_transits/2)

2118 I PLOT TEXT, AT max_transits,0:STR \$(max_transits)

2119 | WINDOW \#13

2120 | SET WINDOW 0,max_rt,0,1

2121 I SET TEXT JUSTIFY "center","bottom"

2122 I SET COLOR 15

2123 I PLOT TEXT, AT 0,0:"0"

2124 I PLOT TEXT, AT max_rt/2,0:STR $\$\left(\max \_r t / 2\right)$

2125 I PLOT TEXT, AT max_rt,0:STR \$(max_rt)

2126 END SUB

2127

2128

2129

2130

2131

2132 SUB variable_change

2133 I SET MODE "80"

2134 I CLEAR

2135 I SET CURSOR 1,1

2136 I PRINT "************************** RESONATE VERSION 1.0 | | $* * * * * * * * * * * * * * * * * * * * * * "$

2137 | PRINT

2138 I PRINT"

COMMENT INPUT DEFAULT VARIABLE I I NAME"

2139 I PRINT "M1 Type [S,PAR,PL,F,T] - . -:";TAB(41);m1\$;TAB(51);

I) "m_1 $\$=\{S, P A R, P L, F, T\} "$

2140 I PRINT "M1 [S,PAR,F,T] R. of Curv.(m):";

I I TAB(40);USING\$("+\#\#.\#\#\#",radius1);TAB(51);"radius1=\{\#\}"

2141 I PRINT "M1 [F,T] \# of Fresnel Zones -:";TAB(40);Zones1;

I । TAB(51);"zones1=\{\#\}"

2142 I PRINT "M1 [F,T] \# of Tiers per Zone :";TAB(40);tpz1;TAB(51);"tpz1=\{\#\}" 
2143 I PRINT "M1 [F,T] \# of outer Tiers - -:";TAB(40);outer_tiers1;TAB(51);

I | "outer_tiers $1=\{\#\}$ "

2144 I PRINT "M1 [S,PAR,PL] Radial Dim.(mm):";TAB(40);

I I USING\$("\#\#.\#\#\#",max_radius1/1e-3);TAB(51);"max_radius1=\{\#\}"

2145 I PRINT

2146 I PRINT "M2 Type \{S,PAR,PL,F,T\} - - -:";TAB(41);m2\$;TAB(51);

I I "m_2\$=\{S,PAR,PL,F,T $\} "$

2147 I PRINT "M2 [S,PAR,F,T] R. of Curv.(m):";TAB(40);

I I USING\$("+\#\#.\#\#\#",radius2);TAB(51);"radius2=\{\#\}"

2148 I PRINT "M2 [F,T] \# of Fresnel Zones -:";TAB(40);zones2;

1 । TAB(51);"zones2=\{\#\}"

2149 I PRINT "M2 [F,T] \# of Tiers per Zone :";TAB(40);tpz2;TAB(51);"tpz2=\{\#\}"

2150 I PRINT "M2 [F,T] \# of outer Tiers - -:";TAB(40);outer_tiers2;TAB(51);

I I "outer_tiers $2=\{\#\}$ "

2151 I PRINT "M2 [S,PAR,PL] Radial Dim.(mm):";TAB(40);USING\$("\#\#.\#\#\#", I । max_radius $2 / 1 \mathrm{e}-3) ; \mathrm{TAB}(51) ; " \max \_$radius $2=\{\#\} "$

2152 I PRINT

2153 I PRINT "Wavelength (um) - . - . - - .:";TAB(40);USING\$("\#\#.\#\#\#", I । lambda/1e-6);TAB(51);"lambda $=\{\#\}$ "

2154 I PRINT "Cavity Length (m) - . - . - :";TAB(40);USING\$("\#\#.\#\#\#",

I I cavity_length);TAB(51);"cavity_length $=\{\#\}$ "

2155 I PRINT "\# of Round Trips, ie RT - - -:";TAB(40);max_rt;TAB(51);

I I "max_rt=\{\#\}"

2156 I PRINT "\# of Radial Increments - - -:";TAB(40);incr;TAB(51);"incr=\{\#\}"

2157 I PRINT "\# of Azimuthal increments - -:";TAB(40);inct;TAB(51);"inct=\{\#\} "

2158 I PRINT "Input Wave \{PL,GAUSS \} - - - -:";TAB(41);input_wave\$;TAB(51);

I I "input_wave $=\{$ PL,GAUSS $\}$ "

2159 | rrow $=4$

2160 । DO

2161 | । SELECT CASE rrow

2162 | । CASE IS $<=4$

2163 | | | rrow $=4$

2164 | | | SET CURSOR rrow,1

2165 | | | PRINT erase_line\$

2166 | । I SET CURSOR rrow, 1

2167 I I I PRINT "M1 Type [S,PAR,PL,F,T]- - -:";TAB(41);m1\$;TAB(51);

11 | | "m_1\$=\{S,PAR,PL,F,T $\} "$

2168 I । I SET CURSOR rrow, 1

2169 I I I SET COLOR "black/white"

2170 । । । PRINT "M1 Type [S,PAR,PL,F,T] - . -:";TAB(41);m1\$

2171 | I | SET COLOR "white/black"

2172 | | | SELECT CASE $\mathrm{m} 1 \$$

2173 | | | CASE "SPHERICAL"

2174 ||। m_1\$="S" 
2175 | । I CASE "PARABOLIC"

2176|||| $\mathrm{m} \_1$ \$ ="PAR"

2177 । $\mid$ । CASE "PLANE"

2178 | $11 \mid \mathrm{m} \_1 \$=" P L "$

2179 । । । CASE "TIERED"

2180 | | | | m_1\$="T"

2181 | । | CASE "FRESNEL"

2182 | | | | m_1\$="F"

2183 । । । CASE ELSE

2184 | । । END SELECT

2185 | । । m_1\$=UCASE\$(input_string\$(rrow,32,"S","PAR","PL","F","T",

| । । | "Enter either S,PAR,PL,F, or T please",m_1\$))

2186 | | | SELECT CASE m_1\$

2187 । । CASE "S"

2188 | | | $\mathrm{m} 1 \$=$ ="SPHERICAL"

2189 I । । CASE "PAR"

2190 | | | | m1\$="PARABOLIC"

2191 | । । CASE "PL"

2192 | | | 1 1\$="PLANE"

2193 । । । CASE "T"

2194 | । । | m1\$="TIERED"

2195 । । । CASE "F"

2196 | | | 1 1\$="FRESNEL"

2197 I । I CASE ELSE

2198 | । | END SELECT

2199 । । । SET CURSOR 4,1

2200 | | | PRINT erase_line\$

2201 I I I SET CURSOR 4,1

2202 I । I PRINT "M1 Type [S,PAR,PL,F,T] - - -:";TAB(32);m_1\$;

I । । T TAB(41);m1\$;TAB(51);"m_1\$=\{S,PAR,PL,F,T $\} "$

2203 | | CASE 5

2204 I । I SET CURSOR rrow, 1

2205 | | | PRINT erase_line\$

2206 | I | SET CURSOR rrow, 1

2207 | । | PRINT "M1 [S,PAR,F,T] R. of Curv.(m):";

I I I I TAB(40);USING $("+\# \#$ \#\#\#",radius1);TAB(51);"radius 1=\{\#\}"

2208 I I I SET CURSOR rrow, 1

2209 I । I SET COLOR "black/white"

2210 I । I PRINT "M1 [S,PAR,F,T] R. of Curv.(m):";

I । । । TAB(40);USING\$("+\#.\#\#\#",radius1)

2211 I I I SET COLOR "white/black"

2212 | । | temp=input_num(rrow,32,radius1)

2213 । । I IF temp=0 then

2214 | | | CALL zero 
2215 | $|1|$ rrow=5

2216 | । । ELSEIF temp $<0$ and (m1 $\$=" F R E S N E L " ~ O R ~ m 1 \$=" T I E R E D ")$ then

2217 । । । I SET CURSOR 2,1

2218 । । | S SET COLOR "black/white"

2219 । । । । PRINT "Must be POSITIVE when $\mathrm{m} 1 \$$ is either

। । । । । FRESNEL or TIERED, Cr to continue:";

22201111 CALL input_

2221 | । | | SET CURSOR 2,1

2222 I । । SET COLOR "white/black"

2223 | | | | PRINT erase_line\$

2224 | $1 \mid$ rrow=5

2225 । । 1 ELSE

2226 | 1 | $\mid$ radius $1=$ temp

2227 | $\mid$ | END IF

2228 | । | SET CURSOR 5,1

2229 | । I PRINT erase_line\$

2230 । I I SET CURSOR 5,1

2231 I । I PRINT "M1 [S,PAR,F,T] R. of Curv.(m):";TAB(31);USING\$("+\#\#.\#\#\#",

। । | | radius1);TAB(40);USING\$("+\#\#.\#\#\#",radius1);TAB(51);"radius $1=\{\#\} "$

2232 | । CASE 6

2233 I I I SET CURSOR rrow,1

2234 | | | PRINT erase_line\$

2235 I । I SET CURSOR rrow, 1

2236 । । । PRINT "M1 [F,T] \# of Zones - . - - -:";TAB(40);Zones1;

। । | TAB(51);"zones $1=\{\#\} "$

2237 | I I SET CURSOR rrow,1

2238 । । I SET COLOR "black/white"

2239 । । । PRINT "M1 [F,T] \# of Zones - . . - -:";TAB(40);zones1

2240 । I I SET COLOR "white/black"

2241 | | | zones1=INT(ABS(input_num(rrow,32,zones1)))

2242 | । । SET CURSOR 6,1

2243 | | | PRINT erase_line\$

2244 I I I SET CURSOR 6,1

2245 I । । PRINT "M1 [F,T] \# of Zones - . . - -:";TAB(31);zones1;TAB(40);zones1;

I । । TAB(51);"zones1= $\{\#\} "$

2246 | । CASE 7

2247 | । | SET CURSOR rrow, 1

2248 | । I PRINT erase_line\$

2249 | । । SET CURSOR rrow, 1

2250 । । । PRINT "M1 [F,T] \# of Tiers per Zone :";TAB(40);tpz1;

I । । TAB(51);"tpz1=\{\#\}"

2251 | । I SET CURSOR rrow, 1

2252 | | | SET COLOR "black/white"

2253 । I। PRINT "M1 [F,T] \# of Tiers per Zone :";TAB(40);tpz1 
2254 I । I SET COLOR "white/black"

2255 | । | tpz1=INT(ABS(input_num(rrow,32,tpz1)))

2256 | । । IF MOD(tpz1,2) $=0$ then

! Odd \# of tiers only.

2257 | । I | SET CURSOR 2,1

2258 | । । । SET COLOR "black/white"

2259 | । । । PRINT "Must be an ODD number, Cr to continue:";

2260 । । । I CALL input_

2261 । । । | SET CURSOR 2,1

2262 I । I I SET COLOR "white/black"

2263 | | | | PRINT erase_line\$

2264 | । | | rrow=7

2265 | | | END IF

2266 । I I SET CURSOR 7,1

2267 | | | PRINT erase_line\$

2268 । I I SET CURSOR 7,1

2269 I । । PRINT "M1 [F,T] \# of Tiers per Zone :";TAB(31);tpz1;TAB(40);tpz1;

I । । T TAB(51);"tpz1=\{\#\}"

2270 | | CASE 8

2271 I । I SET CURSOR rrow, 1

2272 I | I PRINT erase_line\$

2273 I । I SET CURSOR rrow, 1

2274 I । I PRINT "M1 [F,T] \# of outer Tiers - -:";TAB(40);outer_tiers1;

I । । TAB(51);"outer_tiers $1=\{\#\}$ "

2275 I । I SET CURSOR rrow, 1

2276 | । I SET COLOR "black/white"

2277 I । । PRINT "M1 [F,T] \# of outer Tiers - -:";TAB(40);outer_tiers1

2278 I । I SET COLOR "white/black"

2279 | | | outer_tiers1=INT(ABS(input_num(rrow,32,outer_tiers1)))

2280 । । I SET CURSOR 8,1

2281 | | | PRINT erase_line\$

2282 । । I SET CURSOR 8,1

2283 । । । PRINT "M1 [F,T] \# of outer Tiers - -:";TAB(31);outer_tiers1;

I । । I TAB(40);outer_tiers1;TAB(51);"outer_tiers1=\{\#\}"

2284 । I CASE 9

2285 | | | SET CURSOR rrow, 1

2286 | | | PRINT erase_line\$

2287 | । I SET CURSOR rrow, 1

2288 | । । PRINT "M1 [S,PAR,PL] Radial Dim.(mm):";TAB(40);

I । । । USING\$("\#\#.\#\#\#",max_radius1/1e-3);TAB(51);"max_radius1=\{\#\}"

2289 I । I SET CURSOR rrow, 1

2290 । । । IF MOD(tpz1,2)=0 then tpz1=tpz1+1 ! Odd \# of tiers only.

2291 । । । IF zones $1=0$ then

2292 | | | | tiers $1=$ outer_tiers 1

2293 | । | ELSE 
2294 । |।| tiers1=tpz1*(zones1-0.5)+0.5+outer_tiers1

! Total tiers.

2295 । । । END IF

2296 | । । IF m_1\$="F" OR m_1\$="T" then

2297 | | । | IF tiers $1>0$ then

2298 । । । । । max_radius1=SQR(ABS(radius1)*(2*tiers1-2)*lambda/2/tpz1

| | | || | +((2*tiers1-2)*lambda/2/tpz1 $\left.)^{\wedge} 2\right)$

2299 । । । । । SET COLOR "black/white"

2300 | । | | | PRINT "M1 [S,PAR,PL] Radial Dim.(mm):";

| । | । | | TAB(40);USING\$("\#\#.\#\#\#",max_radius1/1e-3)

2301 | । । | | SET COLOR "white/black"

2302 | | | | | maxradius1=1e-3*ABS(input_num(rrow,32,max_radius1/1e-3))

2303 I I I I ELSE

2304 । । । । I SET COLOR "black/white"

2305 | । । । । SET CURSOR 2,1

2306 | । । । | PRINT "Both zones1 \& outer_tiers1 MUST NOT equal 0,

। । । । । । Cr to continue:";

2307 | | | | | SET COLOR "white/black"

2308 | | | | | CALL input

2309 | । । | | SET CURSOR 2,1

2310 | | | | | PRINT erase_line\$

2311 | | | | | rrow=6

2312 | । | | END IF

2313 | । | ELSE

2314 | । । | IF tiers $1>0$ then max_radius1=SQR(ABS(radius1)*(2*tiers1-2)

| । I | | *lambda/2/tpz1+((2*tiers $1-2) * l a m b d a / 2 /$ tpz 1$\left.)^{\wedge} 2\right)$

2315 | । । । SET COLOR "black/white"

2316 | । । । PRINT "M1 [S,PAR,PL] Radial Dim.(mm):";

I । । । । TAB(40);USING\$("\#\#.\#\#\#",max_radius1/1e-3)

2317 | । । । SET COLOR "white/black"

2318 | । | | temp=1e-3*ABS(input_num(rrow,32,max_radius 1/1e-3))

2319 । । । । IF temp $=0$ then

2320 । $\mid$ । $\mid$ | CALL zero

2321 | | | | | rrow=9

2322 । $\mid$ । | ELSE

2323 | | | | | max_radius1=temp

2324 1 11 | END IF

2325 | । I END IF

2326 I I I SET CURSOR 9,1

2327 | | | PRINT erase_line\$

2328 । । I SET CURSOR 9,1

2329 I । I PRINT "M1 [S,PAR,PL] Radial Dim.(mm):";TAB(31);

| । | । USING\$("\#\#.\#\#\#",max_radius1/1e-3);TAB(40);

I । । । USING\$("\#\#.\#\#\#",max_radius1/1e-3);TAB(51);"max_radius $1=\{\#\} "$ 
2331 I I I SET CURSOR rrow, 1

2332 | | | PRINT erase_line\$

2333 I I I SET CURSOR rrow,1

2334 I । । PRINT "M2 Type [S,PAR,PL,F,T]- - - -";TAB(41);m2\$;TAB(51);

I I । | "m_2\$=\{S,PAR,PL,F,T $\} "$

2335 I । । SET CURSOR rrow, 1

2336 | । | SET COLOR "black/white"

2337 | । । PRINT "M2 Type [S,PAR,PL,F,T] - - -:";TAB(41);m2\$

2338 I । I SET COLOR "white/black"

2339 I । I SELECT CASE m2\$

2340 | । | CASE "SPHERICAL"

2341 | | | | m_2\$="S"

2342 I I I CASE "PARABOLIC"

2343 | | | | m_2\$="PAR"

2344 । । CASE "PLANE"

2345 | | | | m_2\$="PL"

2346 | । | CASE "TIERED"

2347 | | | | m_2\$="T"

2348 | । | CASE "FRESNEL"

2349 | । । I m_2\$="F"

2350 | | | CASE ELSE

2351 | । | END SELECT

2352 । । । m_2\$=input_string\$(rrow,32,"S","PAR","PL","F","T",

I I । । "Enter either S,PAR,PL,F, or T please",m_2\$)

2353 | I | SELECT CASE m_2\$

2354 । I । CASE "S"

2355 | | | | m2\$="SPHERICAL"

2356 | | | CASE "PAR"

2357 | | | | m2\$="PARABOLIC"

2358 | । | CASE "PL"

2359 | | । | m2\$="PLANE"

2360 I । I CASE "T"

2361 | । | | m2\$="TIERED"

2362 I । । CASE "F"

2363 | | | | m2\$="FRESNEL"

2364 I । । CASE ELSE

2365 । । । END SELECT

2366 I । I SET CURSOR 11,1

2367 | | | PRINT erase_line\$

2368 I । I SET CURSOR 11,1

2369 I । I PRINT "M2 Type [S,PAR,PL,F,T]- - -:":TAB(32);m_2\$;

I । । I TAB(41);m2\$;TAB(51);"m_2\$=\{S,PAR,PL,F,T $\}$ "

2370 I I CASE 12

2371 | I I SET CURSOR rrow,1 
2372 | | | PRINT erase_line\$

2373 I I I SET CURSOR rrow, 1

2374 I । | PRINT "M2 [S,PAR,F,T] R. of Curv.(m):";

I I । । TAB(40);USING\$("+\#\#.\#\#\#",radius2);TAB(51);"radius2=\{\#\}"

2375 | । I SET CURSOR rrow, 1

2376 I I I SET COLOR "black/white"

2377 I I I PRINT "M2 [S,PAR,F,T] R. of Curv.(m):";

I I । I TAB(40);USING\$("+\#\#.\#\#\#",radius2)

2378 | । । SET COLOR "white/black"

2379 | । | temp=input_num(rrow,32,radius2)

2380 । । । IF temp $=0$ then

2381 | । I I CALL zero

2382 | । | | rrow $=12$

2383 | | | ELSEIF temp $<0$ and (m2\$="FRESNEL" OR $m 2 \$=" T I E R E D ")$ then

2384 I । । I SET CURSOR 2,1

2385 I । । I SET COLOR "black/white"

2386 I । । I PRINT "Must be POSITIVE when m2\$ is either

I । । । । FRESNEL or TIERED, $\mathrm{Cr}$ to continue:";

2387 । I । CALL input_

2388 । । । । SET CURSOR 2,1

2389 I । I I SET COLOR "white/black"

2390 | | | | PRINT erase_line\$

2391 | | | | rrow $=12$

2392 I I I ELSE

2393 | | | | radius2=temp

2394 । । । END IF

2395 I I I SET CURSOR 12,1

2396 | | | PRINT erase_line\$

2397 I I I SET CURSOR 12,1

2398 I I I PRINT "M2 [S,PAR,F,T] R. of Curv.(m):";TAB(31);USING\$("+\#\#.\#\#\#", I । । I radius2);TAB(40);USING\$("+\#\#.\#\#\#",radius2);TAB(51);"radius2=\{\#\}"

2399 I I CASE 13

2400 I I I SET CURSOR rrow, 1

2401 | | | PRINT erase_line $\$$

2402 I | | SET CURSOR rrow, 1

2403 I । । PRINT "M2 [F,T] \# of Zones - - - -:";TAB(40);zones2;TAB(51);

I । । "zones $2=\{\#\}$ "

2404 I | I SET CURSOR rrow, 1

2405 I I I SET COLOR "black/white"

2406 । । । PRINT "M2 [F,T] \# of Zones - . - - :";TAB(40);zones2

2407 I I I SET COLOR "white/black"

2408 | । | zones2=INT(ABS(input_num(rrow,32,zones2)))

2409 I । I SET CURSOR 13,1

2410 | | | PRINT erase_line\$ 
2411 I I I SET CURSOR 13,1

2412 । । । PRINT "M2 [F,T] \# of Zones - . - -:";TAB(31);zones2;TAB(40);zones2;

| । । | TAB(51);"zones2=\{\# $\}$ "

2413 | | CASE 14

2414 | | | SET CURSOR rrow, 1

2415 | | | PRINT erase_line\$

2416 | | | SET CURSOR rrow, 1

2417 । । । PRINT "M2 [F,T] \# of Tiers per Zone :";TAB(40);tpz2;

1 । । TAB(51);"tpz2= $=\#\} "$

2418 । I | SET CURSOR rrow, 1

2419 I I I SET COLOR "black/white"

2420 । । । PRINT "M2 [F,T] \# of Tiers per Zone :";TAB(40);tpz2

2421 | । | SET COLOR "white/black"

2422 | | | tpz2=INT(ABS(input_num(rrow,32,tpz2)))

2423 | । । IF MOD $($ tpz 2,2$)=0$ then

! Odd \# of tiers only.

2424 । । । I SET CURSOR 2,1

2425 । । । I SET COLOR "black/white"

2426 I । । । PRINT "Must be an ODD number, Cr to continue:";

2427 I । I I CALL input_

2428 । । । I SET CURSOR 2,1

2429 I । । I SET COLOR "white/black"

2430 | | | | PRINT erase_line\$

2431 | | । rrow $=14$

2432 | | | END IF

2433 I । I SET CURSOR 14,1

2434 | | | PRINT erase_line\$

2435 I । I SET CURSOR 14,1

2436 I । । PRINT "M2 [F,T] \# of Tiers per Zone :";TAB(31);tpz2;TAB(40);tpz2;

I । । TAB(51);"tpz2=\{\#\}"

2437 | | CASE 15

2438 I | | SET CURSOR rrow, 1

2439 I I I PRINT erase_line\$

2440 | I | SET CURSOR rrow, 1

2441 I । । PRINT "M2 [F,T] \# of outer Tiers - -:";TAB(40);outer_tiers2;TAB(51);

I | | | "outer_tiers $2=\{\#\}$ "

2442 I । I SET CURSOR rrow, 1

2443 | । I SET COLOR "black/white"

2444 | । | PRINT "M2 [F,T] \# of outer Tiers - -:";TAB(40);outer_tiers2

2445 | । | SET COLOR "white/black"

2446 | | | outer_tiers2=INT(ABS(input_num(rrow,32,outer_tiers2)))

2447 I I I SET CURSOR 15,1

2448 | | | PRINT erase_line\$

2449 I । I SET CURSOR 15,1

2450 I । I PRINT "M2 [F,T] \# of outer Tiers - -:";TAB(31);outer_tiers2;TAB(40); 
| | | | outer_tiers2;TAB(51);"outer_tiers2=\{\# $\}$

2452 I | I SET CURSOR rrow, 1

2453 | | | PRINT erase_line $\$$

2454 | | | SET CURSOR rrow, 1

2455 I । । PRINT "M2 [S,PAR,PL] Radial Dim.(mm):";TAB(40);

। । । । USING\$("\#\#.\#\#\#",max_radius2/1e-3);TAB(51);"max_radius2 $=\{\#\} "$

2456 I I I SET CURSOR rrow, 1

2457 । । । IF MOD(tpz2,2)=0 then tpz2=tpz2+1

! Odd \# of tiers only.

2458 | । । IF zones $2=0$ then

2459 | | | | tiers2=outer_tiers2

2460 | । । ELSE

2461 | । । | tiers2=tpz $2 *($ zones2-0.5)+0.5+outer_tiers2

! Total tiers.

2462 | | | END IF

2463 | । । IF $\mathrm{m}_{-} 2 \$=" \mathrm{~F}$ " OR $\mathrm{m} \_2 \$=" T "$ then

2464 | । | | IF tiers $2>0$ then

2465 | । । | | max_radius2=SQR(ABS(radius2)*(2*tiers2-2)*lambda/2/tpz2

||||||$\left.+((2 * \text { tiers } 2-2) * \text { lambda/2/tpz } 2)^{\wedge} 2\right)$

2466 | | । | SET COLOR "black/white"

2467 | । । । । PRINT "M2 [S,PAR,PL] Radial Dim.(mm):";TAB(40);

| 1 | 1 | | USING\$("\#\#.\#\#\#",max_radius2/1e-3)

2468 | | | | | SET COLOR "white/black"

2469 | | | | | maxradius2=1e-3*ABS(input_num(rrow,32,max_radius2/1e-3))

2470 | | | | ELSE

2471 | |।| | SET COLOR "black/white"

2472 | | | | | SET CURSOR 2,1

2473 | | | | | PRINT "Both zones1 \& outer_tiers1 MUST NOT equal 0,

| । । । | | Cr to continue:";

2474 । | । । SET COLOR "white/black"

2475 I $\mid$ । $\mid$ । CALL input_

2476 | । । | | SET CURSOR 2,1

2477 | | | | | PRINT erase_line\$

2478 | | | | rrow $=13$

2479 | | । | END IF

2480 । । I ELSE

2481 । । । । IF tiers $2>0$ then max_radius2=SQR(ABS(radius2)*(2*tiers2-2)*lambda

| | | | | $/ 2 /$ tpz2+((2*tiers $2-2)^{*}$ lambda/2/tpz2 $\left.)^{\wedge} 2\right)$

2482 I । | | SET COLOR "black/white"

2483 । । । । PRINT "M2 [S,PAR,PL] Radial Dim.(mm):";

I । I | । TAB(40);USING\$("\#\#.\#\#\#",max_radius2/1e-3)

2484 I । I I SET COLOR "white/black"

2485 | | | | temp=1e-3*ABS(input_num(rrow,32,max_radius2/1e-3))

2486 | । । । IF temp=0 then

2487 | | | | | CALL zero 
2488 | | | | rrow=16

2489 । 1 । । ELSE

2490 | । । । । max_radius2=temp

2491 I । । I END IF

2492 | | | END IF

2493 I I I SET CURSOR 16,1

2494 I | | PRINT erase_line\$

2495 I | । SET CURSOR 16,1

2496 I । । PRINT "M2 [S,PAR,PL] Radial Dim.(mm):";TAB(31);USING\$("\#\#.\#\#\#",

I । । । max_radius2/1e-3);TAB(40);USING\$("\#\#.\#\#\#",max_radius2/1e-3);

| । । | TAB(51);"max_radius2 $=\{\#\} "$

2497 | । CASE 18

2498 । | | SET CURSOR rrow, 1

2499 | | | PRINT erase_line\$

2500 | । I SET CURSOR rrow, 1

2501 I । I PRINT "Wavelength (um) - - . - - -:";TAB(40);USING\$("\#\#.\#\#\#",

| । । । lambda/le-6);TAB(51);"lambda $=\{\#\} "$

2502 I । । SET CURSOR rrow, 1

2503 I I I SET COLOR "black/white"

2504 I । । PRINT "Wavelength (um) - . . . - - -:";TAB(40);USING\$("\#\#\#\#\#",

| | | | lambda/1e-6)

2505 | । | SET COLOR "white/black"

2506 | | | temp=1e-6*ABS(input_num(rrow,32,lambda/1e-6))

2507 । । I IF temp $=0$ then

2508 | । | | CALL zero

2509 । । । | rrow $=18$

2510 । । । ELSE

2511 | | | | lambda=temp

2512 | | | END IF

2513 I । I SET CURSOR 18,1

2514 I I I PRINT erase_line\$

2515 I I I SET CURSOR 18,1

2516 । । । PRINT "Wavelength (um) - . - . - - -:";TAB(31);USING\$("\#\#.\#\#\#",

I । । । lambda/1e-6);TAB(40);USING\$("\#\#\#\#\#",lambda/1e-6);

2517 । | CASE 19

2518 | | | SET CURSOR rrow, 1

2519 | | | PRINT erase_line\$

2520 । । । SET CURSOR rrow, 1

2521 | । । PRINT "Cavity Length (m) - - - - -:":TAB(40);USING\$("\#\#.\#\#\#",

I । । I cavity_length);TAB(51);"cavity_length $=\{\#\}$ "

2522 । I I SET CURSOR rrow, 1

2523 I । I SET COLOR "black/white"

2524 I I I PRINT "Cavity Length (m) - - - . - -:";TAB(40);USING\$("\#\#.\#\#\#", 
I I I | cavity_length)

2525 | । | SET COLOR "white/black"

2526 | | | temp=ABS(input_num(rrow,32,cavity_length))

2527 | । I IF temp $=0$ then

2528 | । । 1 CALL zero

2529 | । । | rrow=19

2530 | | | ELSE

2531 | | | | cavity_length=temp

2532 | | | END IF

2533 | । I SET CURSOR 19,1

2534 | | | PRINT erase_line\$

2535 I । I SET CURSOR 19,1

2536 I I I PRINT "Cavity Length (m) - - . - - -:";TAB(31);USING\$("\#\#.\#\#\#",

I । । । cavity_length);TAB(40);USING\$("\#\#.\#\#\#",cavity_length);

I । । I TAB(51);"cavity_length $=\{\#\}$ "

2537 | | CASE 20

2538 | | | SET CURSOR rrow, 1

2539 | | | PRINT erase_line\$

2540 | | | SET CURSOR rrow, 1

2541 | । | PRINT "\# of Round Trips, ie RT - - -:";TAB(40);max_rt;TAB(51);

| | | | "max_rt=\{\#\}"

2542 | | | SET CURSOR rrow, 1

2543 I । I SET COLOR "black/white"

2544 I I I PRINT "\# of Round Trips, ie RT - - -:";TAB(40);max_rt

2545 । । । SET COLOR "white/black"

2546 | | | temp=INT(ABS(input_num(rrow,32,max_rt)))

2547 । । । IF temp $=0$ then

2548 | | | | CALL zero

2549 | | | | rrow $=20$

2550 | | | ELSE

2551 | | | | max_rt=temp

2552 | | | END IF

2553 | । I SET CURSOR 20,1

2554 | I I PRINT erase_line\$

2555 | । | SET CURSOR 20,1

2556 I I । PRINT "\# of Round Trips, ie RT - - -:";TAB(31);max_rt;TAB(40);max_rt;

I । | | TAB $(51) ; " m a x \_r t=\{\#\} "$

2557 | | CASE 21

2558 । । । SET CURSOR rrow, 1

2559 | | | PRINT erase_line\$

2560 | I I SET CURSOR rrow, 1

2561 | । I PRINT "\# of Radial Increments - - -:";TAB(40);incr;TAB(51);"incr=\{\#\}"

2562 | | | SET CURSOR rrow, 1

2563 | | | SET COLOR "black/white" 
2564 | । | PRINT "\# of Radial Increments - - -:";TAB(40);incr

2565 | । । SET COLOR "white/black"

2566 | | | temp=INT(ABS(input_num(rrow,32,incr)))

2567 | $\mid$ । IF temp $=0$ then

2568 | । | | CALL zero

2569 | । | | rrow $=21$

2570 | । | ELSEIF MOD(temp,2)=1 then

2571 | | | | SET CURSOR 2,1

2572 । । । । SET COLOR "black/white"

2573 | । । । PRINT "Must be an EVEN number, Cr to continue:";

2574 | । । I CALL input_

2575 । । । | SET CURSOR 2,1

2576 | I | । SET COLOR "white/black"

2577 | | | | PRINT erase_line\$

2578 | । | | rrow $=21$

2579 । । | ELSE

2580 | || $\mid$ incr=temp

2581 | । । END IF

2582 I । I SET CURSOR 21,1

2583 | । | PRINT erase_line\$

2584 । । । SET CURSOR 21,1

2585 I । । PRINT "\# of Radial Increments - - -:";TAB(31);incr;TAB(40);incr;

I। । $\mathrm{TAB}(51) ; "$ incr $=\{\#\} "$

2586 | | CASE 22

2587 I । I SET CURSOR rrow, 1

2588 I । I PRINT erase_line\$

2589 । । । SET CURSOR rrow, 1

2590 I I I PRINT "\# of Azimuthal increments - -:";TAB(40);inct;TAB(51);"inct=\{\#\}"

2591 | । I SET CURSOR rrow, 1

2592 I । I SET COLOR "black/white"

2593 I । I PRINT "\# of Azimuthal increments - -:";TAB(40);inct

2594 I । I SET COLOR "white/black"

2595 | । | temp=INT(ABS(input_num(rrow,32,inct)))

2596 । । । IF temp=0 then

2597 | | | | CALL zero

2598 | | | | rrow=22

2599 | । | ELSEIF MOD(temp,2)=1 then

2600 I । । I SET CURSOR 2,1

2601 | । | | SET COLOR "black/white"

2602 | । । । PRINT "Must be an EVEN number, Cr to continue:";

2603 I I । | CALL input_

2604 । । । | SET CURSOR 2,1

2605 I । I | SET COLOR "white/black"

2606 | | | | PRINT erase_line\$ 
2607 | | | | rrow $=22$

2608 | । | ELSE

2609 I । I I inct=temp

2610 | | | END IF

2611 | | | SET CURSOR 22,1

2612 | | | PRINT erase_line\$

2613 I | | SET CURSOR 22,1

2614 I । I PRINT "\# of Azimuthal increments - -:";TAB(31);inct;TAB(40);inct;

I । । TAB(51);"inct= $\{\#\}$ "

2615 I I CASE 23

2616 | । I SET CURSOR rrow, 1

2617 | | | PRINT erase_line\$

2618 | I I SET CURSOR rrow, 1

2619 I । I PRINT "Input Wave \{PL,GAUSS \} - - -:";TAB(41);input_wave\$;

I I । I TAB(51);"input_wave $\$=\{$ PL,GAUSS $\}$ "

2620 I I I SET CURSOR rrow, 1

2621 I । I SET COLOR "black/white"

2622 I I I PRINT "Input Wave \{PL,GAUSS \} - - - -:"TAB(41);input_wave\$

2623 I I I SET COLOR "white/black"

2624 | । । IF input_wave $\$=" P L A N E "$ then inputwave $\$=" P L "$ ELSE inputwave $\$=$ 1 । । । "GAUSS"

2625 I । । inputwave\$=UCASE\$(input_string\$(rrow,32,"PL","GAUSS",

। । । । "PL","PL","PL","Enter either PL or GAUSS please",inputwave\$))

2626 I । I IF inputwave\$="PL" then input_wave\$="PLANE" ELSE input_wave\$= । । । । "GAUSSIAN"

2627 | । I SET CURSOR 23,1

2628 | । I PRINT erase_line\$

2629 I । I SET CURSOR 23,1

2630 I । I PRINT "Input Wave \{PL,GAUSS \} - - -:";TAB(32);inputwave\$;

I । | | TAB(41);input_wave\$;TAB(51);"input_wave\$=\{PL,GAUSS $\}$ "

2631 | | CASE IS >=24

2632 | | | rrow $=24$

2633 | | | ।

2634 | । | IF m1 \$="PLANE" and m2\$="PLANE" then

2635 | । | । resonator\$="PLANE-PARALLEL"

2636 | । | | confined\$="PLANE-PARALLEL"

2637 | । I | SET CURSOR 2,1

2638 I I । I SET COLOR "black/white"

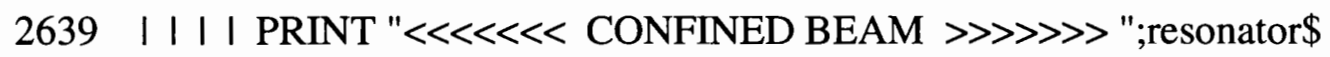

2640|||| $\mid$

2641 | | | ELSEIF m1\$="PLANE" then

2642 | । | | $\mathrm{i}=(1-2 * \text { cavity_length/radius } 2)^{\wedge} 2$

2643 I I I I IF $\mathrm{i}<0$ OR $\mathrm{i}>1$ then

2644 | । । | | resonator $\$=" "$ 
2645 | | | | | confined $\$=" \mathrm{~N} "$

2646 | | | | | SET CURSOR 2,1

2647 | । । । SET COLOR "black/white"

2648|||||| PRINT "<<<<<< UNCONFINED BEAM $\gg \gg \gg \gg>>$;

2649 | । | | ELSEIF cavity_length=radius2 then

2650 | । । | | resonator\$="HALF-CONCENTRIC"

2651 | | | | | confined\$="HALF-CONCENTRIC"

2652 | । । | | SET CURSOR 2,1

2653 | | | | | SET COLOR "black/white"

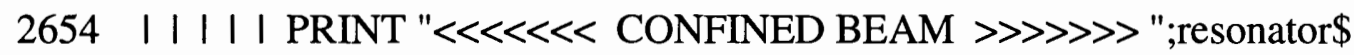

2655 | | | | ELSEIF $2 *$ cavity_length=radius 2 then

2656 | । । । | resonator\$="HALF-CONFOCAL"

2657 | । | । | confined\$="Y"

2658 । $\mid$ । । SET CURSOR 2,1

2659 | | | | | SET COLOR "black/white"

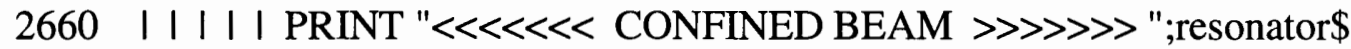

2661 | | | | ELSE

2662 | | | | | resonator $\$=" "$

2663 | | | | | confined $\$=" Y "$

2664 | | | | | SET CURSOR 2,1

2665 । । । | SET COLOR "black/white"

2666 | | | | | PRINT "<<<<<<< CONFINED BEAM $\gg \gg \gg \gg>" ;$

2667 | । । I END IF

2668|||| $\mid$

2669 | | | ELSEIF m2\$="PLANE" then

2670 | | | $\mathrm{i}=\left(1-2^{*} \text { cavity_length/radius } 1\right)^{\wedge} 2$

2671 | । | | IF i $<0$ OR $i>1$ then

2672 | | | | | resonator $\$=" "$

2673 | | | । | confined\$="N"

2674 । $\mid$ । । | SET CURSOR 2,1

2675 । । । । | SET COLOR "black/white"

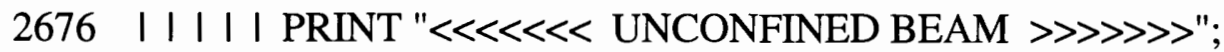

2677 | | | | ELSEIF cavity_length=radius1 then

2678 | | | | | resonator\$="HALF-CONCENTRIC"

2679 | | | | | confined $\$=" H A L F-C O N C E N T R I C "$

2680 । $\mid$ । । । SET CURSOR 2,1

2681 | । | | | SET COLOR "black/white"

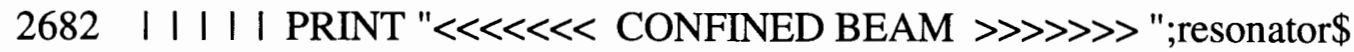

2683 | । | | ELSEIF $2 *$ cavity_length=radius 1 then

2684 | । । । । resonator\$="HALF-CONFOCAL"

2685 | | | | | confined\$="Y"

2686 । $\mid$ । | SET CURSOR 2,1

2687 । । । । । SET COLOR "black/white"

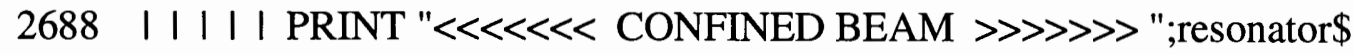


2689 । $\mid$ । $\mathrm{ELSE}$

2690 | $111 \mid$ resonator $\$=" "$

2691 | | | | | confined $\$=" Y "$

2692 | | । | | SET CURSOR 2,1

2693 | । । | | SET COLOR "black/white"

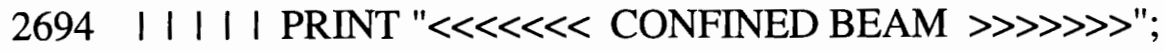

2695 । $\mid$ । $\mid$ END IF

2696 | | | |

2697 | | | ELSEIF (cavity_length=radius1) and (cavity_length=radius2) then

2698 | । । । resonator $\$=$ "CONFOCAL"

2699 | । । | confined $\$=" Y "$

2700 | | | | SET CURSOR 2,1

2701 | । | | SET COLOR "black/white"

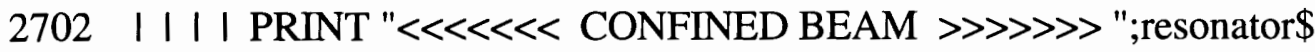

2703 11। 11

2704 | | | ELSEIF (cavity_length/2=radius1) and (cavity_length/2=radius2) then

2705 | । | | resonator $\$=" C O N C E N T R I C "$

2706 | | | | confined\$="CONCENTRIC"

2707 I । । I SET CURSOR 2,1

2708 । । । I SET COLOR "black/white"

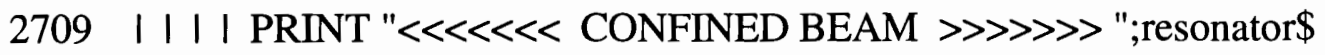

2710 | 1111

2711 | | | ELSEIF (1-cavity_length/radius1)*(1-cavity_length/radius2) $<0$ \&

2712 | | । | \&OR (1-cavity_length/radius1)*(1-cavity_length/radius 2$)>1$ then

2713 | । | | resonator $\$="$ "

2714 | । | | confined $\$=" N "$

2715 । । । SET CURSOR 2,1

2716 । । | | SET COLOR "black/white"

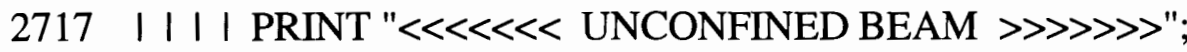

2718 | । | । ।

2719 | । | ELSE

2720 1 1 | 1 resonator $\$=" "$

2721 | । | | confined $\$=" Y "$

2722 । $\mid$ । । SET CURSOR 2,1

2723 | । | | SET COLOR "black/white"

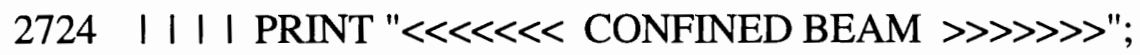

2725 । । । END IF

2726 1 111

2727 I I I SET COLOR "white/black"

2728 I | I SET CURSOR rrow, 1

2729 | | | PRINT erase_line\$

2730 I I | SET CURSOR rrow, 1

2731 । । । PRINT "READY? - . . . . . . . . - "TAB(51);"b\$=\{Y/N\}"

2732 I I I SET CURSOR rrow, 1 
2733 I I I SET COLOR "black/white"

2734 I I । PRINT "READY? - . - . : :"TAB(41);"N"

2735 I I I SET COLOR "white/black"

2736 | | | b\$=UCASE\$(input_string\$(rrow,32,"Y","N","y","n","n",

| | | | "Y/N ENTRY PLS","N"))

2737 I | | SET CURSOR 24,1

2738 | | | PRINT erase_line\$

2739 | | | SET CURSOR 24,1

2740 । । I PRINT "* READY? - - - - :";TAB(32);b\$TAB(41);b\$;

| | | | $\mathrm{TAB}(51) ; " \mathrm{~b} \$=\{\mathrm{Y} / \mathrm{N}\} "$

2741 | | | IF b $\$=" Y "$ then

2742 | | | | EXIT DO

2743 | | | ELSE

2744 | | | | SET CURSOR 2,1

2745 | | | | PRINT erase_line\$

2746 | | | END IF

2747 | । END SELECT

2748 I LOOP

2749 I SET CURSOR 2,1

2750 I SET COLOR "black/white"

2751 I PRINT "<<<<<< ONE MOMENT PLEASE $\gg \gg \gg>$ > ;

2752 I SET COLOR "white/black"

2753 I SET MODE "vga"

2754 END SUB

$2755 \quad$ I

2756

2757

!***************************************************************!

2758

2759

2760

!

SUBROUTINE INPUT_:Gets a line of input.

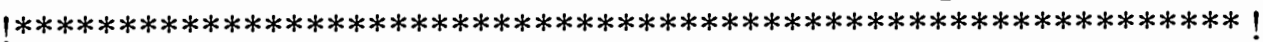

2761 | LINE INPUT PROMPT "':a\$

2762 I a $\$=$ UCASE $\$(\mathrm{a} \$)$

2763 END SUB

2764

2765

2766

2767

2768

2769

$$
1
$$

SUB input

2770 | SET CURSOR 2,1

2771 I SET COLOR "black/white"

2772 I PRINT "Zero is a non-valid variable input, $\mathrm{Cr}$ to continue:";

2773 | CALL input_

2774 | SET CURSOR 2,1 
2775 | SET COLOR "white/black"

2776 I PRINT erase_line\$

2777 END SUB

2778 ।

2779

!*************************************************************!

$2780 !$ SUBROUTINE SIMPSONS_RULE1: Perform a single integration. !

2781

!**************************************************************!

2782

2783 SUB Simpsons_Rule1 (arr(),dn,inc, gral)

2784 I gral1, gral2=0

2785 I FOR iv=2 to inc-1 STEP 2

!!!

2786 | । grall=gral1+arr(iv)

2787 | NEXT iv

2788 | FOR iv=3 to inc-2 STEP 2

2789 | । gral2=gral2+arr(iv)

2790 I NEXT iv

2791 I gral= $(\operatorname{arr}(1)+\operatorname{arr}(\mathrm{inc})+4 * \operatorname{gral} 1+2 * \operatorname{gral} 2) * \mathrm{dn} / 3$

2792 END SUB

2793

2794

2795

2796

2797

2798

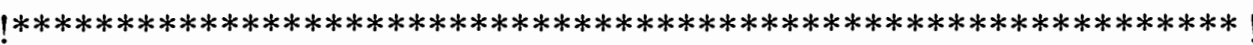

! SUBROUTINE SIMPSONS_RULE2: Perform a double integration. !

!****************************************************************!

1

2799 | real1,real2,imag1, imag2 $=0$

2800 | FOR iv $=2$ to inc- 1 STEP 2

2801 I I real1=real1+re(iv)

2802 I I imag $1=i m a g 1+$ im(iv)

2803 I NEXT iv

2804 I FOR iv $=3$ to inc- 2 STEP 2

2805 | | real2=real2+re(iv

2806 I I imag2=imag2+im(iv)

2807 | NEXT iv

2808 I real=(re(1)+re(inc) $+4 *$ real $1+2 *$ real 2$) * \mathrm{dn} / 3$

2809 । $\mathrm{imag}=(\mathrm{im}(1)+\mathrm{im}(\mathrm{inc})+4 * \mathrm{imag} 1+2 * \mathrm{imag} 2) * \mathrm{dn} / 3$ !!

2810 END SUB

2811 ।

2812 END 
APPENDIX B

CROSS-REFERENCE MAP

FOR COMPUTER PROGRAM "RESONATE VERSION 1.0" 
a

$a \$$

a1\$

a2\$

a3\$

a4\$

a5\$

alternate\$

amp

arr

auto_scale\$

b\$

bot_top

cav_length

cav_length

cavity_length $\begin{array}{lllllllll}405 & 406 & 409 & 413 & 417 & 418 & 435 & 453 & 461\end{array}$

$\begin{array}{lllllllll}462 & 463 & 468 & 472 & 473 & 474 & 484 & 518 & 519\end{array}$

$\begin{array}{lllllllll}520 & 523 & 537 & 538 & 539 & 544 & 548 & 549 & 550\end{array}$

$\begin{array}{lllllllll}553 & 554 & 558 & 584 & 585 & 588 & 592 & 596 & 597\end{array}$

$\begin{array}{lllllllll}614 & 632 & 640 & 641 & 642 & 647 & 651 & 652 & 653\end{array}$

$\begin{array}{lllllllll}663 & 697 & 698 & 699 & 702 & 716 & 717 & 718 & 723\end{array}$

$\begin{array}{llllll}727 & 728 & 729 & 732 & 733 & 737\end{array}$

$\begin{array}{lllllllll}55 & 55 & 56 & 112 & 112 & 113 & 113 & 114 & 114\end{array}$

$\begin{array}{lllllll}114 & 114 & 114 & 115 & 2761 & 2762 & 2762\end{array}$

$85 \quad 114$

$85 \quad 114$

$85 \quad 114$

$85 \quad 114$

$85 \quad 114$

$\begin{array}{lllllllll}210 & 271 & 271 & 586 & 586 & 589 & 589 & 598 & 598\end{array}$

$\begin{array}{lll}615 & 615 & 1003\end{array}$

$\begin{array}{lllllllll}1122 & 1123 & 1124 & 1311 & 1312 & 1313 & 1314 & 1324 & 1325\end{array}$

$\begin{array}{llllll}1326 & 1327 & 1336 & 1337 & 1338 & 1339\end{array}$

$\begin{array}{lllll}2783 & 2786 & 2789 & 2791 & 2791\end{array}$

$\begin{array}{lllllll}216 & 273 & 273 & 1597 & 1617 & 1637 & 1657\end{array}$

$\begin{array}{llll}2736 & 2740 & 2740 & 2741\end{array}$

$\begin{array}{llllllll}387 & 389 & 400 & 400 & 566 & 568 & 579 & 579\end{array}$

$\begin{array}{lllllllll}766 & 767 & 767 & 769 & 778 & 779 & 779 & 781 & 792\end{array}$

$\begin{array}{lllllllll}793 & 793 & 795 & 803 & 804 & 804 & 806 & 876 & 877\end{array}$

$\begin{array}{lllllllll}877 & 879 & 888 & 889 & 889 & 891 & 902 & 903 & 903\end{array}$

$\begin{array}{lllll}905 & 913 & 914 & 914 & 916\end{array}$

$\begin{array}{lllllll}1103 & 1109 & 1110 & 1111 & 1111 & 1113 & 1116\end{array}$

$\begin{array}{lllllllll}188 & 766 & 767 & 768 & 768 & 777 & 777 & 778 & 779\end{array}$

$\begin{array}{lllllllll}780 & 780 & 783 & 783 & 791 & 791 & 792 & 793 & 794\end{array}$

$\begin{array}{lllllllll}794 & 796 & 796 & 802 & 802 & 803 & 804 & 805 & 805\end{array}$

$\begin{array}{lllllllll}807 & 807 & 822 & 876 & 877 & 878 & 878 & 887 & 887\end{array}$

$\begin{array}{lllllllll}888 & 889 & 890 & 890 & 893 & 893 & 901 & 901 & 902\end{array}$

$\begin{array}{lllllllll}903 & 904 & 904 & 906 & 906 & 912 & 912 & 913 & 914\end{array}$

$\begin{array}{lllllllll}915 & 915 & 917 & 917 & 1034 & 1041 & 1055 & 1086 & 1087\end{array}$

$\begin{array}{llllllllll}1103 & 1105 & 1105 & 1107 & 1107 & 1195 & 1224 & 2154 & 2521\end{array}$

$\begin{array}{lllllllll}2524 & 2526 & 2531 & 2536 & 2536 & 2642 & 2649 & 2655 & 2670\end{array}$

$\begin{array}{lllllllll}2677 & 2683 & 2697 & 2697 & 2704 & 2704 & 2711 & 2711 & 2712\end{array}$

2712

$\begin{array}{lllllll}\text { cavity_length_temp } & 822 & 823 & 823 & 824 & 825 & 826\end{array}$

col $\begin{array}{llllllll}24 & 25 & 30 & 36 & 42 & 48 & 85 & 86\end{array}$

color

$\begin{array}{lllllllll}1142 & 1187 & 1187 & 1188 & 1188 & 1388 & 1408 & 1414 & 1434\end{array}$

$\begin{array}{llllllllll}763 & 873 & 2636 & 2645 & 2651 & 2657 & 2663 & 2673 & 2679\end{array}$

$\begin{array}{llllll}2685 & 2691 & 2699 & 2706 & 2714 & 2721\end{array}$ 
cos_kr

cos_t

daf

date\$

default

default\$

depth1

depth2

dev

dev_amp

diameter\$

dn

dr1

dr2

dt

erase_line\$

eta

etch_depth

etch_depth\$

etch_depth1

etch_depth1\$

etch_depth2

etch_depth $2 \$$

EXTEXT\$

EXTYPE

flood_cent

fzt_focus $\begin{array}{lllllllll}1212 & 1213 & 1214 & 1241 & 1242 & 1243 & 1269 & 1270 & 1271\end{array}$

$\begin{array}{lll}1295 & 1296 & 1297\end{array}$

$\begin{array}{lllllll}15 & 263 & 382 & 1208 & 1237 & 1265 & 1291\end{array}$

$\begin{array}{lllllllll}487 & 488 & 494 & 500 & 503 & 504 & 507 & 510 & 511\end{array}$

$\begin{array}{lllllllll}666 & 667 & 673 & 679 & 682 & 683 & 686 & 689 & 690\end{array}$

$\begin{array}{lllllll}1091 & 1091 & 1091 & 1383 & 1383 & 1383\end{array}$

$\begin{array}{lllllllll}24 & 29 & 31 & 35 & 37 & 41 & 43 & 47 & 49\end{array}$

$67 \quad 76$

$\begin{array}{lllllllll}85 & 90 & 91 & 95 & 96 & 100 & 101 & 105 & 106\end{array}$

$125 \quad 135$

$\begin{array}{lllllllll}15 & 260 & 308 & 322 & 465 & 476 & 476 & 479 & 479\end{array}$

$480 \quad 480$

$\begin{array}{lllllllll}15 & 260 & 360 & 374 & 644 & 655 & 655 & 658 & 658\end{array}$

$659 \quad 659$

$\begin{array}{llll}215 & 1355 & 1355 & 1355\end{array}$

$\begin{array}{llll}1348 & 1352 & 1354 & 1355\end{array}$

$\begin{array}{llll}2089 & 2090 & 2091 & 2092\end{array}$

$\begin{array}{lllll}2783 & 2791 & 2798 & 2808 & 2809\end{array}$

$\begin{array}{lllllllll}284 & 326 & 336 & 404 & 416 & 460 & 471 & 487 & 490\end{array}$

$\begin{array}{lllllllll}498 & 517 & 536 & 547 & 816 & 817 & 837 & 838 & 1113\end{array}$

$\begin{array}{lllllllll}1113 & 1116 & 1116 & 1132 & 1135 & 1201 & 1218 & 1222 & 1275\end{array}$

$\begin{array}{lllllllll}1416 & 1424 & 1427 & 1430 & 1431 & 1449 & 1488 & 1562 & 1565\end{array}$

$\begin{array}{lllllllll}284 & 336 & 378 & 583 & 595 & 639 & 650 & 666 & 669\end{array}$

$\begin{array}{lllllllll}677 & 696 & 715 & 726 & 926 & 927 & 935 & 936 & 1193\end{array}$

$\begin{array}{lllllllll}1230 & 1247 & 1301 & 1390 & 1398 & 1401 & 1404 & 1405 & 1504\end{array}$

$\begin{array}{llllll}1543 & 1552 & 1555 & 1571 & 1574\end{array}$

$\begin{array}{llllll}380 & 382 & 1216 & 1245 & 1273 & 1299\end{array}$

$\begin{array}{lllllllll}66 & 75 & 124 & 134 & 217 & 2165 & 2200 & 2205 & 2223\end{array}$

$\begin{array}{lllllllll}2229 & 2234 & 2243 & 2248 & 2263 & 2267 & 2272 & 2281 & 2286\end{array}$

$\begin{array}{lllllllll}2310 & 2327 & 2332 & 2367 & 2372 & 2390 & 2396 & 2401 & 2410\end{array}$

$\begin{array}{lllllllll}2415 & 2430 & 2434 & 2439 & 2448 & 2453 & 2477 & 2494 & 2499\end{array}$

$\begin{array}{lllllllll}2514 & 2519 & 2534 & 2539 & 2554 & 2559 & 2577 & 2583 & 2588\end{array}$

$\begin{array}{llllllll}2606 & 2612 & 2617 & 2628 & 2729 & 2738 & 2745 & 2776\end{array}$

$\begin{array}{lll}1110 & 1113 & 1116\end{array}$

$\begin{array}{llllll}277 & 329 & 407 & 410 & 586 & 589\end{array}$

$\begin{array}{lll}278 & 330 & 1057\end{array}$

$\begin{array}{lll}300 & 308 & 322\end{array}$

$301 \quad 1070$

$\begin{array}{lll}352 & 360 & 374\end{array}$

$353 \quad 1073$

$71 \quad 130$

$\begin{array}{lll}59 & 71 & 130\end{array}$

$\begin{array}{llllll}394 & 398 & 561 & 573 & 577 & 740\end{array}$

$\begin{array}{llllllll}281 & 333 & 779 & 779 & 780 & 889 & 889 & 890\end{array}$ 
145

gim

$\operatorname{gim} 2$

gral

gral1

gral2

gre

gre2

grid_graph1\$

grid_graph $2 \$$

$\mathrm{h}$

$\begin{array}{lllllllll}11 & 261 & 1214 & 1216 & 1243 & 1245 & 1271 & 1273 & 1297\end{array}$

$\begin{array}{lll}1299 & 11 & 261\end{array}$

$\begin{array}{llllllll}1216 & 1218 & 1245 & 1247 & 1273 & 1275 & 1299 & 1301\end{array}$

$2783 \quad 2791$

$\begin{array}{llll}2784 & 2786 & 2786 & 2791\end{array}$

$\begin{array}{llll}2784 & 2789 & 2789 & 2791\end{array}$

$\begin{array}{lllllllll}11 & 261 & 1213 & 1216 & 1242 & 1245 & 1270 & 1273 & 1296\end{array}$

1299

$\begin{array}{lllllllll}11 & 261 & 1216 & 1218 & 1245 & 1247 & 1273 & 1275 & 1299\end{array}$

1301

$850 \quad 1444$

$949 \quad 1499$

$\begin{array}{lllllllll}1210 & 1213 & 1214 & 1239 & 1242 & 1243 & 1267 & 1270 & 1271\end{array}$

$\begin{array}{lll}1293 & 1296 & 1297\end{array}$

$\begin{array}{lllllllll}154 & 155 & 156 & 279 & 280 & 281 & 282 & 286 & 287\end{array}$

$\begin{array}{lllllllll}288 & 290 & 293 & 294 & 296 & 297 & 297 & 297 & 298\end{array}$

$\begin{array}{lllllllll}299 & 306 & 308 & 308 & 322 & 322 & 324 & 331 & 332\end{array}$

$\begin{array}{lllllllll}333 & 334 & 338 & 339 & 340 & 342 & 345 & 346 & 348\end{array}$

$\begin{array}{lllllllll}349 & 349 & 349 & 350 & 351 & 358 & 360 & 360 & 374\end{array}$

$\begin{array}{lllllllll}374 & 376 & 381 & 382 & 382 & 383 & 403 & 404 & 407\end{array}$

$\begin{array}{lllllllll}407 & 410 & 410 & 414 & 415 & 416 & 420 & 421 & 422\end{array}$

$\begin{array}{lllllllll}422 & 423 & 424 & 427 & 428 & 429 & 429 & 430 & 431\end{array}$

$\begin{array}{lllllllll}437 & 438 & 439 & 439 & 440 & 441 & 444 & 445 & 446\end{array}$

$\begin{array}{lllllllll}446 & 447 & 448 & 454 & 459 & 460 & 465 & 469 & 470\end{array}$

$\begin{array}{lllllllll}471 & 476 & 477 & 478 & 478 & 479 & 480 & 485 & 489\end{array}$

$\begin{array}{lllllllll}490 & 492 & 494 & 496 & 497 & 498 & 500 & 501 & 502\end{array}$

$\begin{array}{lllllllll}502 & 503 & 504 & 507 & 508 & 509 & 509 & 510 & 511\end{array}$

$\begin{array}{lllllllll}514 & 516 & 517 & 520 & 524 & 527 & 528 & 529 & 530\end{array}$

$\begin{array}{lllllllll}535 & 536 & 545 & 546 & 547 & 550 & 551 & 552 & 552\end{array}$

$\begin{array}{lllllllll}553 & 554 & 559 & 582 & 583 & 586 & 586 & 589 & 589\end{array}$

$\begin{array}{lllllllll}593 & 594 & 595 & 599 & 600 & 601 & 601 & 602 & 603\end{array}$

$\begin{array}{lllllllll}606 & 607 & 608 & 608 & 609 & 610 & 616 & 617 & 618\end{array}$

$\begin{array}{lllllllll}618 & 619 & 620 & 623 & 624 & 625 & 625 & 626 & 627\end{array}$

$\begin{array}{lllllllll}633 & 638 & 639 & 644 & 648 & 649 & 650 & 655 & 656\end{array}$

$\begin{array}{lllllllll}657 & 657 & 658 & 659 & 664 & 668 & 669 & 671 & 673\end{array}$

$\begin{array}{lllllllll}675 & 676 & 677 & 679 & 680 & 681 & 681 & 682 & 683\end{array}$

$\begin{array}{lllllllll}686 & 687 & 688 & 688 & 689 & 690 & 693 & 695 & 696\end{array}$

$\begin{array}{lllllllll}699 & 703 & 706 & 707 & 708 & 709 & 714 & 715 & 724\end{array}$

$\begin{array}{lllllllll}725 & 726 & 729 & 730 & 731 & 731 & 732 & 733 & 738\end{array}$

$\begin{array}{lllllllll}746 & 748 & 748 & 750 & 752 & 754 & 754 & 756 & 815\end{array}$

$\begin{array}{lllllllll}816 & 816 & 817 & 817 & 818 & 836 & 837 & 837 & 838\end{array}$

$\begin{array}{lllllllll}838 & 839 & 856 & 858 & 858 & 860 & 862 & 864 & 864\end{array}$

$\begin{array}{lllllllll}866 & 925 & 926 & 926 & 927 & 927 & 928 & 934 & 935\end{array}$

$\begin{array}{lllllllll}935 & 936 & 936 & 937 & 950 & 962 & 1112 & 1113 & 1113\end{array}$ 
$\begin{array}{lllllllll}1113 & 1114 & 1115 & 1116 & 1116 & 1116 & 1117 & 1121 & 1122\end{array}$

$\begin{array}{lllllllll}1122 & 1123 & 1125 & 1131 & 1132 & 1133 & 1133 & 1133 & 1134\end{array}$

$\begin{array}{llllllllll}1310 & 1311 & 1311 & 1312 & 1314 & 1315 & 1323 & 1324 & 1324\end{array}$

$\begin{array}{lllllllll}1325 & 1327 & 1328 & 1335 & 1336 & 1336 & 1337 & 1339 & 1340\end{array}$

$\begin{array}{llllllllll}1389 & 1390 & 1390 & 1390 & 1391 & 1403 & 1404 & 1404 & 1405\end{array}$

$\begin{array}{llllllllll}1405 & 1406 & 1415 & 1416 & 1416 & 1416 & 1417 & 1429 & 1430\end{array}$

$\begin{array}{llllllllll}1430 & 1431 & 1431 & 1432 & 1448 & 1449 & 1449 & 1449 & 1450\end{array}$

$\begin{array}{llllllllll}1467 & 1468 & 1469 & 1469 & 1471 & 1472 & 1472 & 1474 & 1474\end{array}$

$\begin{array}{llllllllll}1477 & 1478 & 1488 & 1489 & 1503 & 1504 & 1504 & 1504 & 1505\end{array}$

$\begin{array}{lllllllll}1522 & 1523 & 1524 & 1524 & 1526 & 1527 & 1527 & 1529 & 1529\end{array}$

$\begin{array}{llllllllll}1532 & 1533 & 1543 & 1544 & 1551 & 1552 & 1553 & 1553 & 1553\end{array}$

$\begin{array}{llllllllll}1554 & 1561 & 1562 & 1563 & 1563 & 1563 & 1564 & 1570 & 1571\end{array}$

$\begin{array}{lllllllll}1572 & 1572 & 1572 & 1573 & 1598 & 1599 & 1602 & 1603 & 1604\end{array}$

$\begin{array}{lllllllll}1606 & 1618 & 1619 & 1622 & 1623 & 1624 & 1626 & 1638 & 1639\end{array}$

$\begin{array}{llllllllll}1642 & 1643 & 1644 & 1646 & 1658 & 1659 & 1662 & 1663 & 1664\end{array}$

$\begin{array}{lllllllll}1666 & 1689 & 1690 & 1690 & 1691 & 1693 & 1695 & 1695 & 1697\end{array}$

$\begin{array}{lllllllll}1697 & 1698 & 1707 & 1708 & 1708 & 1709 & 1713 & 1714 & 1714\end{array}$

$\begin{array}{lllllllll}1715 & 1719 & 1720 & 1720 & 1721 & 1757 & 1758 & 1758 & 1759\end{array}$

$\begin{array}{llllllllll}1761 & 1763 & 1763 & 1765 & 1765 & 1766 & 1775 & 1776 & 1776\end{array}$

$\begin{array}{lllllllll}1777 & 1780 & 1781 & 1781 & 1782 & 1785 & 1786 & 1786 & 1787\end{array}$

$\begin{array}{lllllllll}1844 & 1845 & 1845 & 1846 & 1853 & 1854 & 1855 & 1856 & 1864\end{array}$

$\begin{array}{lllllllll}1865 & 1865 & 1866 & 1906 & 1907 & 1907 & 1908 & 1915 & 1916\end{array}$

$\begin{array}{lllllllll}1917 & 1918 & 1926 & 1927 & 1927 & 1928 & 1954 & 1955 & 1956\end{array}$

$\begin{array}{llllllllll}1980 & 1981 & 1981 & 1982 & 1984 & 1986 & 1986 & 1988 & 1988\end{array}$

$\begin{array}{lllllllll}1989 & 2001 & 2002 & 2002 & 2003 & 2006 & 2007 & 2007 & 2008\end{array}$

$\begin{array}{lllllllll}2011 & 2012 & 2012 & 2013 & 2031 & 2032 & 2032 & 2033 & 2039\end{array}$

$\begin{array}{lllllllll}2040 & 2041 & 2042 & 2050 & 2051 & 2051 & 2052 & 2060 & 2061\end{array}$

$\begin{array}{lllllllll}2061 & 2062 & 2068 & 2069 & 2070 & 2071 & 2079 & 2080 & 2080\end{array}$

$\begin{array}{lllllll}2081 & 2642 & 2643 & 2643 & 2670 & 2671 & 2671\end{array}$

ii

iii

im

im1

im1_n1

im1_n2

im2

imag $\begin{array}{lllllllll}539 & 540 & 541 & 718 & 719 & 720 & 1466 & 1467 & 1467\end{array}$

$\begin{array}{lllll}1491 & 1521 & 1522 & 1522 & 1546\end{array}$

$\begin{array}{lllllllll}745 & 747 & 747 & 747 & 748 & 751 & 753 & 753 & 753\end{array}$

$\begin{array}{lllllllll}754 & 855 & 857 & 857 & 857 & 858 & 861 & 863 & 863\end{array}$

$863 \quad 864$

$\begin{array}{lllll}2798 & 2802 & 2806 & 2809 & 2809\end{array}$

$\begin{array}{lllllll}14 & 262 & 1150 & 1206 & 1235 & 1263 & 1289\end{array}$

$\begin{array}{llllll}1206 & 1213 & 1214 & 1263 & 1270 & 1271\end{array}$

$\begin{array}{lllllll}1235 & 1242 & 1243 & 1289 & 1296 & 1297\end{array}$

$\begin{array}{lllllllll}14 & 262 & 1101 & 1116 & 1122 & 1133 & 1150 & 1218 & 1247\end{array}$

$\begin{array}{lllllllll}1275 & 1301 & 1311 & 1324 & 1336 & 1390 & 1416 & 1449 & 1458\end{array}$

$\begin{array}{llllllllll}1460 & 1461 & 1463 & 1469 & 1471 & 1472 & 1474 & 1504 & 1513\end{array}$

$\begin{array}{lllllllll}1515 & 1516 & 1518 & 1524 & 1526 & 1527 & 1529 & 1553 & 1563\end{array}$

1572

$2798 \quad 2809$ 


\begin{tabular}{|c|c|c|c|c|c|c|c|c|c|}
\hline imag1 & 2799 & 2802 & 2802 & 2809 & & & & & \\
\hline imag2 & 2799 & 2806 & 2806 & 2809 & & & & & \\
\hline inc & 2783 & 2785 & 2788 & 2791 & 2798 & 2800 & 2804 & 2808 & 2809 \\
\hline \multirow[t]{22}{*}{ incr } & 191 & 254 & 254 & 255 & 255 & 255 & 255 & 256 & 256 \\
\hline & 257 & 257 & 257 & 257 & 260 & 261 & 261 & 262 & 262 \\
\hline & 262 & 262 & 263 & 263 & 263 & 274 & 284 & 326 & 336 \\
\hline & 378 & 387 & 389 & 403 & 415 & 415 & 415 & 421 & 428 \\
\hline & 438 & 445 & 457 & 457 & 457 & 457 & 457 & 459 & 470 \\
\hline & 470 & 470 & 477 & 487 & 489 & 497 & 497 & 497 & 500 \\
\hline & 501 & 503 & 504 & 508 & 516 & 527 & 531 & 535 & 535 \\
\hline & 546 & 546 & 546 & 551 & 566 & 568 & 582 & 594 & 594 \\
\hline & 594 & 600 & 607 & 617 & 624 & 636 & 636 & 636 & 636 \\
\hline & 636 & 638 & 649 & 649 & 649 & 656 & 666 & 668 & 676 \\
\hline & 676 & 676 & 679 & 680 & 682 & 683 & 687 & 695 & 706 \\
\hline & 710 & 714 & 714 & 725 & 725 & 725 & 730 & 743 & 746 \\
\hline & 746 & 751 & 753 & 758 & 758 & 788 & 813 & 815 & 835 \\
\hline & 836 & 849 & 850 & 853 & 856 & 856 & 861 & 863 & 868 \\
\hline & 868 & 898 & 923 & 925 & 933 & 934 & 948 & 949 & 986 \\
\hline & 1112 & 1115 & 1121 & 1127 & 1128 & 1131 & 1135 & 1166 & 1181 \\
\hline & 1192 & 1199 & 1218 & 1221 & 1226 & 1228 & 1247 & 1252 & 1257 \\
\hline & 1275 & 1278 & 1281 & 1283 & 1301 & 1310 & 1317 & 1318 & 1323 \\
\hline & 1330 & 1331 & 1335 & 1342 & 1343 & 1375 & 1389 & 1398 & 1401 \\
\hline & 1403 & 1415 & 1424 & 1427 & 1429 & 1448 & 1466 & 1503 & 1521 \\
\hline & 1551 & 1555 & 1561 & 1565 & 1570 & 1574 & 1950 & 2156 & 2561 \\
\hline & 2564 & 2566 & 2580 & 2585 & 2585 & & & & \\
\hline \multirow[t]{3}{*}{ inct } & 192 & 261 & 261 & 263 & 380 & 381 & 986 & 1207 & 1216 \\
\hline & 1236 & 1245 & 1264 & 1273 & 1290 & 1299 & 2157 & 2590 & 2593 \\
\hline & 2595 & 2609 & 2614 & 2614 & & & & & \\
\hline init & 178 & 232 & & & & & & & \\
\hline initial_int1_amp & 1127 & 1128 & 1128 & 1129 & & & & & \\
\hline initial_int2_amp & 1317 & 1318 & 1318 & 1319 & & & & & \\
\hline initial_power1 & 1135 & 1136 & 1136 & 1137 & 1138 & 1586 & 1591 & & \\
\hline initial_power2 & 1555 & 1556 & 1556 & 1557 & 1558 & 1591 & & & \\
\hline \multirow[t]{2}{*}{ input_ } & 54 & 64 & 73 & 111 & 122 & 132 & 2220 & 2260 & 2308 \\
\hline & 2387 & 2427 & 2475 & 2574 & 2603 & 2760 & 2773 & & \\
\hline \multirow[t]{3}{*}{ input_num } & 24 & 29 & 35 & 41 & 47 & 56 & 67 & 76 & 2212 \\
\hline & 2241 & 2255 & 2279 & 2302 & 2318 & 2379 & 2408 & 2422 & 2446 \\
\hline & 2469 & 2485 & 2506 & 2526 & 2546 & 2566 & 2595 & & \\
\hline \multirow[t]{2}{*}{ input_string\$ } & 85 & 90 & 95 & 100 & 105 & 115 & 125 & 135 & 2185 \\
\hline & 2352 & 2625 & 2736 & & & & & & \\
\hline \multirow[t]{2}{*}{ input_wave\$ } & 193 & 268 & 268 & 1052 & 1099 & 2158 & 2619 & 2622 & 2624 \\
\hline & 2626 & 2626 & 2630 & & & & & & \\
\hline & 143 & & & & & & & & \\
\hline & 144 & 224 & 232 & & & & & & \\
\hline
\end{tabular}




\begin{tabular}{|c|c|c|c|c|c|c|c|c|c|}
\hline inputwave\$ & 2624 & 2624 & 2625 & 2625 & 2626 & 2630 & & & \\
\hline int1_amp & 1330 & 1331 & 1331 & 1348 & 1348 & 1353 & 1884 & 1891 & 1939 \\
\hline int 2 amp & 1342 & 1343 & 1343 & 1824 & 1829 & 1877 & & & \\
\hline \multirow{9}{*}{ intvar_max } & 1834 & 1834 & 1835 & 1835 & 1836 & 1842 & 1842 & 1842 & 1845 \\
\hline & 1848 & 1848 & 1848 & 1848 & 1850 & 1850 & 1854 & 1854 & 1856 \\
\hline & 1857 & 1858 & 1859 & 1870 & 1870 & 1870 & 1870 & 1871 & 1871 \\
\hline & 1878 & 1896 & 1896 & 1897 & 1897 & 1898 & 1904 & 1904 & 1904 \\
\hline & 1907 & 1910 & 1910 & 1910 & 1910 & 1912 & 1912 & 1916 & 1916 \\
\hline & 1918 & 1919 & 1920 & 1921 & 1932 & 1932 & 1932 & 1932 & 1933 \\
\hline & 1933 & 1940 & 2030 & 2030 & 2030 & 2032 & 2036 & 2036 & 2040 \\
\hline & 2040 & 2042 & 2043 & 2044 & 2045 & 2059 & 2059 & 2059 & 2061 \\
\hline & 2065 & 2065 & 2069 & 2069 & 2071 & 2072 & 2073 & 2074 & \\
\hline intvar_max_old & 1878 & 1940 & & & & & & & \\
\hline \multirow[t]{9}{*}{ intvar_min } & 1831 & 1831 & 1832 & 1832 & 1836 & 1842 & 1842 & 1842 & 1845 \\
\hline & 1848 & 1848 & 1850 & 1855 & 1855 & 1856 & 1856 & 1857 & 1858 \\
\hline & 1858 & 1859 & 1870 & 1870 & 1871 & 1879 & 1893 & 1893 & 1894 \\
\hline & 1894 & 1898 & 1904 & 1904 & 1904 & 1907 & 1910 & 1910 & 1912 \\
\hline & 1917 & 1917 & 1918 & 1918 & 1919 & 1920 & 1920 & 1921 & 1932 \\
\hline & 1932 & 1933 & 1941 & 2030 & 2030 & 2030 & 2032 & 2036 & 2041 \\
\hline & 2041 & 2042 & 2042 & 2043 & 2044 & 2044 & 2045 & 2059 & 2059 \\
\hline & 2059 & 2061 & 2065 & 2070 & 2070 & 2071 & 2071 & 2072 & 2073 \\
\hline & 2073 & 2074 & & & & & & & \\
\hline intvar_min_old & 1879 & 1941 & & & & & & & \\
\hline intvar1_max & 1834 & 1884 & 1895 & 1895 & 1896 & & & & \\
\hline intvar1_max_old & 1886 & 1898 & 1940 & & & & & & \\
\hline intvar1_min & 1831 & 1884 & 1892 & 1892 & 1893 & & & & \\
\hline intvar1_min_old & 1885 & 1898 & 1941 & & & & & & \\
\hline \multirow[t]{2}{*}{ intvar1_per_pass } & 13 & 266 & 1884 & 1891 & 1892 & 1895 & 1913 & 1927 & 1934 \\
\hline & 1936 & 1937 & 2037 & 2051 & & & & & \\
\hline intvar2_max & 1824 & 1833 & 1833 & 1835 & 1897 & & & & \\
\hline intvar2_max_old & 1836 & 1878 & 1886 & & & & & & \\
\hline intvar2_min & 1824 & 1830 & 1830 & 1832 & 1894 & & & & \\
\hline intvar2_min_old & 1836 & 1879 & 1885 & & & & & & \\
\hline \multirow[t]{2}{*}{ intvar2_per_pass } & 13 & 266 & 1824 & 1829 & 1830 & 1833 & 1851 & 1865 & 1872 \\
\hline & 1874 & 1875 & 2066 & 2080 & & & & & \\
\hline \multirow[t]{2}{*}{ iv } & 2785 & 2786 & 2787 & 2788 & 2789 & 2790 & 2800 & 2801 & 2802 \\
\hline & 2803 & 2804 & 2805 & 2806 & 2807 & & & & \\
\hline \multirow[t]{7}{*}{ J } & 280 & 281 & 281 & 288 & 290 & 293 & 293 & 332 & 333 \\
\hline & 333 & 340 & 342 & 345 & 345 & 404 & 406 & 409 & 416 \\
\hline & 418 & 435 & 460 & 462 & 471 & 473 & 490 & 492 & 494 \\
\hline & 498 & 500 & 503 & 504 & 507 & 510 & 511 & 517 & 519 \\
\hline & 520 & 536 & 538 & 539 & 547 & 549 & 550 & 553 & 554 \\
\hline & 583 & 585 & 588 & 595 & 597 & 614 & 639 & 641 & 650 \\
\hline & 652 & 669 & 671 & 673 & 677 & 679 & 682 & 683 & 686 \\
\hline
\end{tabular}


$\mathrm{jj}$
$\mathrm{jjj}$
$\mathrm{k}$

key

$\mathrm{kr}$

labels

labels

lambda

last_dev_amp

last_int1_amp

last_int2_amp

last $j$

last_last_dev_amp

last_power

last_power1

last_power2

left

loss_max

loss_max_old

loss_max 1

loss_max2

$\begin{array}{lllllllll}689 & 690 & 696 & 698 & 699 & 715 & 717 & 718 & 726 \\ 728 & 729 & 732 & 733 & 747 & 748 & 748 & 749 & 753 \\ 754 & 754 & 755 & 787 & 788 & 788 & 812 & 813 & 813 \\ 834 & 835 & 835 & 857 & 858 & 858 & 859 & 863 & 864 \\ 864 & 865 & 897 & 898 & 898 & 922 & 923 & 923 & 932 \\ 933 & 933 & 1132 & 1133 & 1397 & 1398 & 1398 & 1400 & 1401 \\ 1401 & 1423 & 1424 & 1424 & 1426 & 1427 & 1427 & 1477 & 1479 \\ 1480 & 1481 & 1481 & 1483 & 1483 & 1487 & 1488 & 1532 & 1534 \\ 1535 & 1536 & 1536 & 1538 & 1538 & 1542 & 1543 & 1552 & 1553 \\ 1562 & 1563 & 1571 & 1572 & & & & & \\ 1458 & 1461 & 1463 & 1477 & 1513 & 1516 & 1518 & 1532 & \\ 1469 & 1472 & 1474 & 1477 & 1524 & 1527 & 1529 & 1532 & \\ 275 & 1113 & 1116 & 1170 & 1185 & 1209 & 1238 & 1266 & 1292 \\ 26 & 27 & 27 & 33 & 39 & 45 & 53 & 55 & 87 \\ 88 & 88 & 93 & 98 & 103 & 110 & 112 & 1953 & 2095 \\ 1209 & 1211 & 1212 & 1238 & 1240 & 1241 & 1266 & 1268 & 1269 \\ 1292 & 1294 & 1295 & & & & & & \\ 964 & 1685 & 1753 & 1840 & 1902 & 1973 & & & \end{array}$

2104

$\begin{array}{lllllllll}189 & 200 & 200 & 207 & 207 & 275 & 277 & 281 & 281\end{array}$

$\begin{array}{lllllllll}293 & 293 & 297 & 297 & 300 & 329 & 333 & 333 & 345\end{array}$

$\begin{array}{lllllllll}345 & 349 & 349 & 352 & 520 & 539 & 550 & 553 & 554\end{array}$

$\begin{array}{lllllllll}699 & 718 & 729 & 732 & 733 & 767 & 779 & 793 & 804\end{array}$

$\begin{array}{lllllllll}828 & 877 & 889 & 903 & 914 & 1034 & 1041 & 1049 & 1055\end{array}$

$\begin{array}{lllllllll}1204 & 1233 & 2153 & 2298 & 2298 & 2314 & 2314 & 2465 & 2465\end{array}$

$\begin{array}{llllllll}2481 & 2481 & 2501 & 2504 & 2506 & 2511 & 2516 & 2516\end{array}$

$\begin{array}{lll}1347 & 1354 & 1355\end{array}$

$\begin{array}{llll}1129 & 1884 & 1891 & 1939\end{array}$

$\begin{array}{llll}1319 & 1824 & 1829 & 1877\end{array}$

$\begin{array}{llllll}1479 & 1480 & 1487 & 1534 & 1535 & 1542\end{array}$

$1347 \quad 1355$

$\begin{array}{llllllll}1138 & 1592 & 1615 & 1616 & 1635 & 1636 & 1655 & 1656\end{array}$

$\begin{array}{llll}1137 & 1613 & 1634 & 1654\end{array}$

$\begin{array}{lllll}1557 & 1594 & 1614 & 1633 & 1653\end{array}$

$\begin{array}{llllll}392 & 396 & 400 & 571 & 575 & 579\end{array}$

$\begin{array}{lllllllll}1596 & 1677 & 1677 & 1678 & 1678 & 1681 & 1687 & 1687 & 1690\end{array}$

$\begin{array}{lllllllll}1693 & 1700 & 1700 & 1702 & 1703 & 1705 & 1725 & 1725 & 1725\end{array}$

$\begin{array}{lllllllll}1725 & 1726 & 1726 & 1749 & 1755 & 1755 & 1758 & 1761 & 1768\end{array}$

$\begin{array}{llllllllll}1768 & 1770 & 1771 & 1773 & 1791 & 1791 & 1791 & 1791 & 1792\end{array}$

$\begin{array}{lllllllll}1792 & 1819 & 1979 & 1979 & 1979 & 1981 & 1984 & 1991 & 1991\end{array}$

$1993 \quad 19941996$

$\begin{array}{llll}1596 & 1681 & 1749 & 1819\end{array}$

$\begin{array}{llllll}1596 & 1642 & 1649 & 1662 & 1669 & 1677\end{array}$

$\begin{array}{lllll}1602 & 1609 & 1622 & 1629 & 1678\end{array}$ 


\begin{tabular}{|c|c|c|c|c|c|c|c|c|c|}
\hline \multirow[t]{5}{*}{ loss_min } & 1595 & 1675 & 1675 & 1676 & 1676 & 1681 & 1687 & 1687 & 1690 \\
\hline & 1693 & 1700 & 1702 & 1703 & 1704 & 1705 & 1725 & 1725 & 1726 \\
\hline & 1749 & 1755 & 1755 & 1758 & 1761 & 1768 & 1770 & 1771 & 1772 \\
\hline & 1773 & 1791 & 1791 & 1792 & 1818 & 1979 & 1979 & 1979 & 1981 \\
\hline & 1984 & 1991 & 1993 & 1994 & 1995 & 1996 & & & \\
\hline loss_min_old & 1595 & 1681 & 1749 & 1818 & & & & & \\
\hline loss_min 1 & 1595 & 1643 & 1648 & 1663 & 1668 & 1675 & & & \\
\hline loss_min2 & 1603 & 1608 & 1623 & 1628 & 1676 & & & & \\
\hline \multirow[t]{2}{*}{ loss_per_pass } & 12 & 265 & 1591 & 1593 & 1615 & 1635 & 1655 & 1708 & 1728 \\
\hline & 1729 & 1746 & 1776 & 1794 & 1795 & 1816 & 2002 & 2024 & \\
\hline \multirow[t]{2}{*}{ loss1_per_pass } & 12 & 264 & 1633 & 1638 & 1639 & 1653 & 1658 & 1659 & 1714 \\
\hline & 1781 & 1798 & 1800 & 1801 & 1811 & 2007 & 2019 & & \\
\hline \multirow[t]{2}{*}{ loss2_per_pass } & 12 & 264 & 1593 & 1598 & 1599 & 1613 & 1618 & 1619 & 1720 \\
\hline & 1731 & 1732 & 1741 & 1786 & 2012 & 2022 & & & \\
\hline \multirow[t]{2}{*}{ m_1\$ } & 2174 & 2176 & 2178 & 2180 & 2182 & 2185 & 2185 & 2186 & 2202 \\
\hline & 2296 & 2296 & & & & & & & \\
\hline \multirow[t]{2}{*}{$\mathrm{m} \_2 \$$} & 2341 & 2343 & 2345 & 2347 & 2349 & 2352 & 2352 & 2353 & 2369 \\
\hline & 2463 & 2463 & & & & & & & \\
\hline \multirow[t]{6}{*}{$\mathrm{m} 1 \$$} & 181 & 269 & 269 & 276 & 285 & 285 & 402 & 455 & 458 \\
\hline & 486 & 486 & 491 & 499 & 515 & 764 & 772 & 776 & 801 \\
\hline & 874 & 882 & 885 & 900 & 989 & 990 & 992 & 1001 & 1008 \\
\hline & 1008 & 1009 & 1054 & 1065 & 1068 & 1077 & 1079 & 1104 & 1396 \\
\hline & 1422 & 2139 & 2167 & 2170 & 2172 & 2188 & 2190 & 2192 & 2194 \\
\hline & 2196 & 2202 & 2216 & 2216 & 2634 & 2641 & & & \\
\hline \multirow[t]{6}{*}{$\mathrm{m} 2 \$$} & 182 & 270 & 270 & 328 & 337 & 337 & 581 & 634 & 637 \\
\hline & 665 & 665 & 670 & 678 & 694 & 764 & 772 & 775 & 790 \\
\hline & 874 & 882 & 886 & 911 & 996 & 997 & 999 & 1001 & 1020 \\
\hline & 1020 & 1021 & 1065 & 1071 & 1077 & 1081 & 1107 & 2146 & 2334 \\
\hline & 2337 & 2339 & 2355 & 2357 & 2359 & 2361 & 2363 & 2369 & 2383 \\
\hline & 2383 & 2634 & 2669 & & & & & & \\
\hline main & 145 & 225 & 231 & & & & & & \\
\hline \multirow[t]{3}{*}{ max_amp } & 1120 & 1124 & 1124 & 1126 & 1309 & 1313 & 1313 & 1314 & 1316 \\
\hline & 1322 & 1326 & 1326 & 1327 & 1329 & 1334 & 1338 & 1338 & 1339 \\
\hline & 1341 & 1390 & 1416 & 1449 & 1504 & & & & \\
\hline \multirow[t]{3}{*}{ max_amp_i } & 1314 & 1327 & 1339 & 1457 & 1458 & 1458 & 1460 & 1461 & 1461 \\
\hline & 1463 & 1463 & 1477 & 1512 & 1513 & 1513 & 1515 & 1516 & 1516 \\
\hline & 1518 & 1518 & 1532 & & & & & & \\
\hline \multirow[t]{3}{*}{ max_radius 1} & 200 & 295 & 299 & 326 & 386 & 389 & 565 & 568 & 812 \\
\hline & 834 & 961 & 1034 & 1426 & 1447 & 1455 & 2089 & 2144 & 2288 \\
\hline & 2298 & 2300 & 2302 & 2314 & 2316 & 2318 & 2323 & 2329 & 2329 \\
\hline \multirow[t]{3}{*}{ max_radius2 } & 207 & 347 & 351 & 378 & 386 & 389 & 565 & 568 & 922 \\
\hline & 932 & 961 & 1041 & 1400 & 1502 & 1510 & 2091 & 2151 & 2455 \\
\hline & 2465 & 2467 & 2469 & 2481 & 2483 & 2485 & 2490 & 2496 & 2496 \\
\hline
\end{tabular}


max_rt

$\begin{array}{lllllllll}190 & 253 & 264 & 264 & 266 & 266 & 1164 & 1179 & 1842\end{array}$

$\begin{array}{llllllllll}1844 & 1844 & 1848 & 1850 & 1853 & 1858 & 1858 & 1861 & 1870\end{array}$

$\begin{array}{lllllllll}1870 & 1871 & 1904 & 1906 & 1906 & 1910 & 1912 & 1915 & 1920\end{array}$

$\begin{array}{lllllllll}1920 & 1923 & 1932 & 1932 & 1933 & 2030 & 2031 & 2031 & 2036\end{array}$

$\begin{array}{lllllllll}2039 & 2044 & 2044 & 2047 & 2059 & 2060 & 2060 & 2065 & 2068\end{array}$

$\begin{array}{lllllllll}2073 & 2073 & 2076 & 2106 & 2110 & 2110 & 2111 & 2111 & 2120\end{array}$

$\begin{array}{lllllllll}2124 & 2124 & 2125 & 2125 & 2155 & 2541 & 2544 & 2546 & 2551\end{array}$

$2556 \quad 2556$

max_transits

$\begin{array}{lllllllll}253 & 265 & 1148 & 1687 & 1689 & 1689 & 1695 & 1697 & 1700\end{array}$

$\begin{array}{lllllllll}1703 & 1703 & 1705 & 1725 & 1726 & 1755 & 1757 & 1757 & 1763\end{array}$

$\begin{array}{lllllllllll}1765 & 1768 & 1771 & 1771 & 1773 & 1791 & 1792 & 1979 & 1980\end{array}$

$\begin{array}{lllllllll}1980 & 1986 & 1988 & 1991 & 1994 & 1994 & 1998 & 2113 & 2117\end{array}$

$\begin{array}{lll}2117 & 2118 & 2118\end{array}$

maxradius 1

2302

2469

maxradius2

$\begin{array}{lllllllll}1151 & 1157 & 1171 & 1251 & 1277 & 1321 & 1333 & 1346 & 1438\end{array}$

$\begin{array}{lllllllll}1493 & 1560 & 1569 & 1612 & 1652 & 1680 & 1748 & 1826 & 1888\end{array}$

$n$

213

n_number

$\begin{array}{llll}1055 & 1060 & 1061 & 1063\end{array}$

n_number 1

$\begin{array}{llll}1034 & 1035 & 1036 & 1038\end{array}$

n_number2

$\begin{array}{lllll}1041 & 1042 & 1043 & 1045\end{array}$

$\mathrm{n} 1$

$\begin{array}{lllllllll}1199 & 1200 & 1200 & 1201 & 1202 & 1203 & 1204 & 1205 & 1206\end{array}$

$\begin{array}{llllllllll}1216 & 1216 & 1217 & 1221 & 1222 & 1224 & 1226 & 1231 & 1232\end{array}$

$\begin{array}{llllllllll}1247 & 1247 & 1248 & 1257 & 1258 & 1259 & 1260 & 1261 & 1262\end{array}$

$\begin{array}{lllllllll}1263 & 1273 & 1273 & 1274 & 1278 & 1281 & 1284 & 1285 & 1301\end{array}$

$1301 \quad 1302$

$\mathrm{n} 2$

$\begin{array}{lllllllll}1192 & 1193 & 1195 & 1197 & 1202 & 1203 & 1218 & 1218 & 1219\end{array}$

$\begin{array}{llllllllll}1228 & 1229 & 1229 & 1230 & 1231 & 1232 & 1233 & 1234 & 1235\end{array}$

$\begin{array}{lllllllll}1245 & 1245 & 1246 & 1252 & 1255 & 1258 & 1259 & 1275 & 1275\end{array}$

$\begin{array}{llllllllll}1276 & 1283 & 1284 & 1285 & 1286 & 1287 & 1288 & 1289 & 1299\end{array}$

12991300

open_viewports $\quad 152 \quad 179 \quad 234$

$\begin{array}{llllllllll}\text { outer_tiers1 } & 186 & 196 & 198 & 242 & 244 & 2143 & 2274 & 2277 & 2279\end{array}$

$\begin{array}{lllllllll}2279 & 2283 & 2283 & 2292 & 2294 & & \end{array}$

$\begin{array}{llllllllll}\text { outer_tiers2 } & 187 & 203 & 205 & 249 & 251 & 2150 & 2441 & 2444 & 2446\end{array}$

$\begin{array}{llllllllll}2446 & 2450 & 2450 & 2459 & 2461 & & & & \end{array}$

$\begin{array}{llllllllll}\mathrm{p} 2 & 488 & 494 & 507 & 510 & 511 & 667 & 673 & 686 & 689\end{array}$

past_int1_amp $\quad 1348 \quad 1353$

phase $\quad 1143 \quad 1170 \quad 1185$

$\begin{array}{lllllllll}\text { phase_delay } & 15 & 260 & 1170 & 1185 & 1477 & 1477 & 1532 & 1532\end{array}$

$\begin{array}{llllllllll}\text { pi } & 275 & 380 & 767 & 779 & 793 & 804 & 828 & 877 & 889\end{array}$

$\begin{array}{lllllllll}903 & 914 & 1136 & 1455 & 1455 & 1461 & 1463 & 1472 & 1474\end{array}$ 
152

power

power 1

power2

q

q1

147

1534

1481

1483

1510

1510

1516

1518

$1527 \quad 1529$

$\begin{array}{lllll}1536 & 1538 & 1556 & 1566 & 1575\end{array}$

$\begin{array}{lllllllll}1558 & 1567 & 1576 & 1586 & 1592 & 1615 & 1616 & 1635 & 1636\end{array}$

16551656

$\begin{array}{llllllll}1565 & 1566 & 1566 & 1567 & 1633 & 1634 & 1653 & 1654\end{array}$

$\begin{array}{llllllll}1558 & 1574 & 1575 & 1575 & 1576 & 1594 & 1613 & 1614\end{array}$

$\begin{array}{llll}463 & 474 & 642 & 653\end{array}$

$\begin{array}{lllllllll}463 & 464 & 464 & 465 & 474 & 475 & 475 & 476 & 479\end{array}$

$\begin{array}{lllllllll}480 & 642 & 643 & 643 & 644 & 653 & 654 & 654 & 655\end{array}$

$658 \quad 659$

r

$\begin{array}{lllllllll}1208 & 1209 & 1210 & 1210 & 1237 & 1238 & 1239 & 1239 & 1265\end{array}$

r1

1266

1267

1267

1291

$\begin{array}{lll}1292 & 1293 & 1293\end{array}$

$\begin{array}{llll}1201 & 1202 & 1202 & 1203 \\ 1204 & 1210 & 1261 & 1267\end{array}$

r1_lambda

$10 \quad 256$

$1223 \quad 1231$

r1_lambda_1

12031208

120

1261

rlr1

r1r22

r1r22_1

r1r22_2

r2

9257

$\begin{array}{llll}9 & 1203 & 1259\end{array}$

$\begin{array}{lllll}9 & 257 & 1232 & 1285\end{array}$

$\begin{array}{lllll}1193 & 1194 & 1194 & 1203\end{array}$

123

1204

1222

1223

$1223 \quad 1232$

$\begin{array}{lllll}1233 & 1239 & 1287 & 1293\end{array}$

r2_lambda_2

r2r2

$\begin{array}{llll}10 & 256 & 1233 & 1287\end{array}$

$1194 \quad 1202$

$\operatorname{rad} 1$

$\operatorname{rad} 2$

radius 1

radius 2

$\begin{array}{lllllll}16 & 258 & 297 & 299 & 519 & 538 & 549\end{array}$

$\begin{array}{lllllll}16 & 258 & 349 & 351 & 698 & 717 & 728\end{array}$

$\begin{array}{lllllll}183 & 200 & 293 & 297 & 391 & 488 & 488\end{array}$

$\begin{array}{llllll}492 & 500 & 500 & 503 & 503 & 504\end{array}$

$\begin{array}{llllll}539 & 539 & 550 & 550 & 553 & 553\end{array}$

$488492 \quad 492$

$\begin{array}{llll}765 & 767 & 767 & 768\end{array}$

793

793

$554 \quad 554 \quad 764$

$\begin{array}{lllllllll}826 & 829 & 829 & 874 & 875 & 877 & 877 & 878 & 914\end{array}$

$\begin{array}{lllllllll}914 & 915 & 1011 & 1011 & 1012 & 1013 & 1013 & 1014 & 1016\end{array}$

$\begin{array}{lllllllll}1105 & 2140 & 2207 & 2210 & 2212 & 2226 & 2231 & 2231 & 2298\end{array}$

$\begin{array}{lllllllll}2314 & 2670 & 2677 & 2683 & 2697 & 2704 & 2711 & 2712\end{array}$

$\begin{array}{lllllllll}183 & 207 & 345 & 349 & 570 & 667 & 667 & 671 & 671\end{array}$

$\begin{array}{lllllllll}671 & 679 & 679 & 682 & 682 & 683 & 683 & 699 & 699\end{array}$

$\begin{array}{lllllllll}718 & 718 & 729 & 729 & 732 & 732 & 733 & 733 & 764\end{array}$

$\begin{array}{lllllllll}765 & 804 & 804 & 805 & 824 & 825 & 826 & 830 & 830\end{array}$

$\begin{array}{lllllllll}874 & 875 & 903 & 903 & 904 & 1023 & 1023 & 1024 & 1025\end{array}$

$\begin{array}{llllllllll}1025 & 1026 & 1028 & 1107 & 2147 & 2374 & 2377 & 2379 & 2393\end{array}$

$\begin{array}{lllllllll}2398 & 2398 & 2465 & 2481 & 2642 & 2649 & 2655 & 2697 & 2704\end{array}$

27112712

re

$\begin{array}{lllll}2798 & 2801 & 2805 & 2808 & 2808\end{array}$

re 1

re1_n1

$\begin{array}{lllllll}14 & 262 & 1149 & 1205 & 1234 & 1262 & 1288\end{array}$

$\begin{array}{llllll}1205 & 1213 & 1214 & 1262 & 1270 & 1271\end{array}$ 


\begin{tabular}{|c|c|c|c|c|c|c|c|c|c|}
\hline re1_n2 & 1234 & 1242 & 1243 & 1288 & 1296 & 1297 & & & \\
\hline \multirow[t]{5}{*}{ re 2} & 14 & 262 & 1100 & 1113 & 1122 & 1133 & 1149 & 1218 & 1247 \\
\hline & 1275 & 1301 & 1311 & 1324 & 1336 & 1390 & 1416 & 1449 & 1457 \\
\hline & 1458 & 1461 & 1463 & 1468 & 1469 & 1472 & 1474 & 1504 & 1512 \\
\hline & 1513 & 1516 & 1518 & 1523 & 1524 & 1527 & 1529 & 1553 & 1563 \\
\hline & 1572 & & & & & & & & \\
\hline real & 2798 & 2808 & & & & & & & \\
\hline reall & 2799 & 2801 & 2801 & 2808 & & & & & \\
\hline real2 & 2799 & 2805 & 2805 & 2808 & & & & & \\
\hline \multirow[t]{3}{*}{ resonator\$ } & 2094 & 2635 & 2639 & 2644 & 2650 & 2654 & 2656 & 2660 & 2662 \\
\hline & 2672 & 2678 & 2682 & 2684 & 2688 & 2690 & 2698 & 2702 & 2705 \\
\hline & 2709 & 2713 & 2720 & & & & & & \\
\hline response\$ & 85 & 120 & & & & & & & \\
\hline right & 393 & 397 & 400 & 572 & 576 & 579 & & & \\
\hline rings 1 & 16 & 259 & 293 & 295 & 462 & 473 & & & \\
\hline rings2 & 16 & 259 & 345 & 347 & 641 & 652 & & & \\
\hline rnd & 310 & 362 & & & & & & & \\
\hline row & 24 & 25 & 30 & 36 & 42 & 48 & 85 & 86 & \\
\hline \multirow[t]{15}{*}{ rrow } & 28 & 28 & 28 & 28 & 28 & 28 & 34 & 34 & 34 \\
\hline & 34 & 34 & 40 & 46 & 57 & 57 & 57 & 57 & 57 \\
\hline & 57 & 89 & 89 & 94 & 94 & 94 & 94 & 94 & 99 \\
\hline & 104 & 116 & 116 & 2159 & 2161 & 2163 & 2164 & 2166 & 2168 \\
\hline & 2185 & 2204 & 2206 & 2208 & 2212 & 2215 & 2224 & 2233 & 2235 \\
\hline & 2237 & 2241 & 2247 & 2249 & 2251 & 2255 & 2264 & 2271 & 2273 \\
\hline & 2275 & 2279 & 2285 & 2287 & 2289 & 2302 & 2311 & 2318 & 2321 \\
\hline & 2331 & 2333 & 2335 & 2352 & 2371 & 2373 & 2375 & 2379 & 2382 \\
\hline & 2391 & 2400 & 2402 & 2404 & 2408 & 2414 & 2416 & 2418 & 2422 \\
\hline & 2431 & 2438 & 2440 & 2442 & 2446 & 2452 & 2454 & 2456 & 2469 \\
\hline & 2478 & 2485 & 2488 & 2498 & 2500 & 2502 & 2506 & 2509 & 2518 \\
\hline & 2520 & 2522 & 2526 & 2529 & 2538 & 2540 & 2542 & 2546 & 2549 \\
\hline & 2558 & 2560 & 2562 & 2566 & 2569 & 2578 & 2587 & 2589 & 2591 \\
\hline & 2595 & 2598 & 2607 & 2616 & 2618 & 2620 & 2625 & 2632 & 2728 \\
\hline & 2730 & 2732 & 2736 & & & & & & \\
\hline \multirow[t]{7}{*}{$\mathrm{rt}$} & 1144 & 1164 & 1172 & 1172 & 1179 & 1613 & 1618 & 1619 & 1653 \\
\hline & 1658 & 1659 & 1713 & 1719 & 1731 & 1731 & 1732 & 1732 & 1741 \\
\hline & 1780 & 1785 & 1798 & 1798 & 1800 & 1800 & 1801 & 1801 & 1811 \\
\hline & 1829 & 1830 & 1833 & 1851 & 1864 & 1872 & 1874 & 1874 & 1875 \\
\hline & 1875 & 1891 & 1892 & 1895 & 1913 & 1926 & 1934 & 1936 & 1936 \\
\hline & 1937 & 1937 & 2006 & 2011 & 2019 & 2022 & 2037 & 2050 & 2066 \\
\hline & 2079 & & & & & & & & \\
\hline $\mathrm{Rz}$ & 1111 & 1113 & 1116 & & & & & & \\
\hline s\$ & 314 & 319 & 321 & 366 & 371 & 373 & & & \\
\hline \multirow[t]{2}{*}{ scale } & 305 & 312 & 312 & 312 & 317 & 317 & 317 & 357 & 364 \\
\hline & 364 & 364 & 369 & 369 & 369 & & & & \\
\hline
\end{tabular}




\begin{tabular}{|c|c|c|c|c|c|c|c|c|c|}
\hline show\$ & 214 & 1197 & 1226 & 1255 & 1281 & & & & \\
\hline \multirow[t]{2}{*}{$\operatorname{sim}$} & 312 & 313 & 313 & 314 & 317 & 318 & 318 & 319 & 322 \\
\hline & 364 & 365 & 365 & 366 & 369 & 370 & 370 & 371 & 374 \\
\hline $\operatorname{sim} \$$ & 304 & 321 & 321 & 356 & 373 & 373 & 1095 & & \\
\hline \multirow[t]{2}{*}{$\operatorname{sim} 1$} & 303 & 313 & 313 & 314 & 318 & 318 & 319 & 355 & 365 \\
\hline & 365 & 366 & 370 & 370 & 371 & & & & \\
\hline similate\$ & 208 & 307 & 359 & 1095 & & & & & \\
\hline Simpsons_Rule 1 & 1127 & 1135 & 1317 & 1330 & 1342 & 1555 & 1565 & 1574 & 2783 \\
\hline Simpsons_Rule2 & 1216 & 1218 & 1245 & 1247 & 1273 & 1275 & 1299 & 1301 & 2798 \\
\hline \multirow[t]{2}{*}{$\sin \_k r$} & 1211 & 1213 & 1214 & 1240 & 1242 & 1243 & 1268 & 1270 & 1271 \\
\hline & 1294 & 1296 & 1297 & & & & & & \\
\hline step_max_radius & 283 & 284 & 335 & 336 & 787 & 897 & 1055 & & \\
\hline step_max_radius 1 & 1423 & & & & & & & & \\
\hline step_max_radius2 & 1397 & & & & & & & & \\
\hline \multirow[t]{2}{*}{ step_rings } & 14 & 265 & 281 & 283 & 333 & 335 & 406 & 409 & 418 \\
\hline & 435 & 585 & 588 & 597 & 614 & & & & \\
\hline \multirow[t]{2}{*}{ step_switch\$ } & 211 & 272 & 272 & 407 & 410 & 419 & 436 & 586 & 586 \\
\hline & 589 & 589 & 598 & 598 & 615 & 615 & & & \\
\hline \multirow[t]{2}{*}{ step_tiers } & 212 & 265 & 279 & 283 & 331 & 335 & 405 & 417 & 584 \\
\hline & 596 & 1059 & & & & & & & \\
\hline \multirow[t]{2}{*}{$\mathrm{t} 1$} & 1207 & 1208 & 1213 & 1214 & 1215 & 1264 & 1265 & 1270 & 1271 \\
\hline & 1272 & & & & & & & & \\
\hline \multirow[t]{2}{*}{ t2 } & 1236 & 1237 & 1242 & 1243 & 1244 & 1290 & 1291 & 1296 & 1297 \\
\hline & 1298 & & & & & & & & \\
\hline \multirow[t]{4}{*}{ temp } & 2212 & 2213 & 2216 & 2226 & 2318 & 2319 & 2323 & 2379 & 2380 \\
\hline & 2383 & 2393 & 2485 & 2486 & 2490 & 2506 & 2507 & 2511 & 2526 \\
\hline & 2527 & 2531 & 2546 & 2547 & 2551 & 2566 & 2567 & 2570 & 2580 \\
\hline & 2595 & 2596 & 2599 & 2609 & & & & & \\
\hline \multirow[t]{3}{*}{ tiers 1} & 196 & 198 & 200 & 200 & 240 & 242 & 244 & 244 & 259 \\
\hline & 286 & 287 & 295 & 461 & 472 & 1078 & 1080 & 2292 & 2294 \\
\hline & 2297 & 2298 & 2298 & 2314 & 2314 & 2314 & & & \\
\hline \multirow[t]{3}{*}{ tiers2 } & 203 & 205 & 207 & 207 & 247 & 249 & 251 & 251 & 259 \\
\hline & 338 & 339 & 347 & 640 & 651 & 1078 & 1082 & 2459 & 2461 \\
\hline & 2464 & 2465 & 2465 & 2481 & 2481 & 2481 & & & \\
\hline time\$ & 1091 & 1383 & & & & & & & \\
\hline tol & 302 & 303 & 312 & 317 & 354 & 355 & 364 & 369 & 1095 \\
\hline \multirow[t]{6}{*}{ tpz1 } & 185 & 194 & 194 & 194 & 198 & 200 & 200 & 239 & 239 \\
\hline & 239 & 240 & 244 & 260 & 293 & 293 & 300 & 301 & 306 \\
\hline & 308 & 308 & 322 & 322 & 463 & 464 & 474 & 475 & 476 \\
\hline & 479 & 480 & 990 & 1067 & 2142 & 2250 & 2253 & 2255 & 2255 \\
\hline & 2256 & 2269 & 2269 & 2290 & 2290 & 2290 & 2294 & 2298 & 2298 \\
\hline & 2314 & 2314 & & & & & & & \\
\hline \multirow[t]{2}{*}{ tpz2 } & 185 & 201 & 201 & 201 & 205 & 207 & 207 & 246 & 246 \\
\hline & 246 & 247 & 251 & 260 & 345 & 345 & 352 & 353 & 358 \\
\hline
\end{tabular}


$\begin{array}{lllllllll}360 & 360 & 374 & 374 & 642 & 643 & 653 & 654 & 655\end{array}$ $\begin{array}{llllllllll}658 & 659 & 997 & 1067 & 2149 & 2417 & 2420 & 2422 & 2422\end{array}$

$\begin{array}{lllllllll}2423 & 2436 & 2436 & 2457 & 2457 & 2457 & 2461 & 2465 & 2465\end{array}$ 24812481

transits

$\begin{array}{lllllllll}1148 & 1151 & 1187 & 1190 & 1191 & 1220 & 1308 & 1347 & 1355\end{array}$

$\begin{array}{lllllllll}1386 & 1412 & 1550 & 1590 & 1615 & 1632 & 1635 & 1655 & 1707\end{array}$

$\begin{array}{lllllllll}1711 & 1728 & 1728 & 1729 & 1729 & 1746 & 1775 & 1794 & 1794\end{array}$

$\begin{array}{lllllllll}1795 & 1795 & 1797 & 1816 & 1823 & 1826 & 1883 & 1944 & 1948\end{array}$

$\begin{array}{lll}1948 & 2001 & 2024\end{array}$

variable_change

$238 \quad 2132$

w0

$\begin{array}{lllllllll}769 & 781 & 782 & 795 & 799 & 806 & 808 & 828 & 831\end{array}$

$\begin{array}{lllllllll}832 & 879 & 891 & 892 & 905 & 909 & 916 & 918 & 1109\end{array}$

$1113 \quad 1116$

w1

$\begin{array}{lllllllll}770 & 782 & 785 & 787 & 797 & 799 & 808 & 810 & 812\end{array}$

$\begin{array}{lllllllll}816 & 817 & 831 & 834 & 837 & 838 & 1419 & 1423 & 1426\end{array}$

$\begin{array}{lllllllll}1430 & 1431 & 832 & 880 & 892 & 895 & 897 & 907 & 909\end{array}$

$\begin{array}{lllllllll}918 & 920 & 922 & 926 & 927 & 932 & 935 & 936 & 1393\end{array}$

$\begin{array}{llllllllll}1397 & 1400 & 1404 & 1405 & & & & & \\ 767 & 769 & 779 & 781 & 785 & 793 & 795 & 797 & 804\end{array}$

waist

$\begin{array}{lllllllll}806 & 810 & 877 & 879 & 889 & 891 & 895 & 903 & 905\end{array}$

$\begin{array}{lllllllll}907 & 914 & 916 & 920 & 1109 & 1113 & 1113 & 1116 & 1116\end{array}$

Z

$\begin{array}{lllllllll}1200 & 1202 & 1202 & 1210 & 1229 & 1231 & 1231 & 1239 & 1260\end{array}$

$\begin{array}{lll}1267 & 1286 & 1293\end{array}$

z_1

$\begin{array}{llll}9 & 254 & 1200 & 1260\end{array}$

$\mathrm{z} \_2$

$9 \quad 254 \quad 1229 \quad 1286$

z_m 1

$829 \quad 831$

$\mathrm{z} \_\mathrm{m} 2$

$830 \quad 832$

$\mathrm{z} 0$

$\begin{array}{lllllllll}768 & 769 & 780 & 781 & 794 & 795 & 805 & 806 & 827\end{array}$

$\begin{array}{lllllllll}828 & 831 & 832 & 878 & 879 & 890 & 891 & 904 & 905\end{array}$

$\begin{array}{lllllll}915 & 916 & 1105 & 1107 & 1109 & 1110 & 1111\end{array}$

$\begin{array}{llllllllll}\text { z0_sqrd } & 823 & 824 & 824 & 825 & 825 & 826 & 826 & 827 & 829\end{array}$

830

$\begin{array}{lllllllll}525 & 528 & 528 & 531 & 533 & 540 & 540 & 550 & 550\end{array}$

$\begin{array}{lllllllll}553 & 553 & 554 & 554 & 712 & 719 & 719 & 729 & 729\end{array}$

$\begin{array}{llllll}732 & 732 & 733 & 733 & 1224 & 1229\end{array}$

z11

$\begin{array}{lllllllll}526 & 529 & 529 & 534 & 541 & 541 & 550 & 553 & 554\end{array}$

$\begin{array}{llllll}713 & 720 & 720 & 729 & 732 & 733\end{array}$

$\mathrm{z} 2$

$\mathrm{z} 22$

$\begin{array}{llllll}704 & 707 & 707 & 710 & 1195 & 1200\end{array}$

$\begin{array}{lll}705 & 708 & 708\end{array}$

zero

$\begin{array}{lllllllll}2214 & 2320 & 2381 & 2487 & 2508 & 2528 & 2548 & 2568 & 2597\end{array}$ 2769

zones1

$\begin{array}{lllllllll}184 & 195 & 198 & 240 & 241 & 258 & 296 & 518 & 537\end{array}$

$\begin{array}{lllllllll}548 & 2141 & 2236 & 2239 & 2241 & 2241 & 2245 & 2245 & 2291\end{array}$ 
zones2

$\mathrm{zr} 1 \mathrm{r} 2$

zr1r2_1

zr1r2_2

$\mathrm{zz} 1$

$\mathrm{zz} 2$

zzm $\begin{array}{lllllllll}184 & 202 & 205 & 247 & 248 & 258 & 348 & 697 & 716\end{array}$ $\begin{array}{lllllllll}727 & 2148 & 2403 & 2406 & 2408 & 2408 & 2412 & 2412 & 2458\end{array}$ 2461

$\begin{array}{llllllll}1202 & 1208 & 1231 & 1237 & 1258 & 1265 & 1284 & 1291\end{array}$

$9 \quad 255 \quad 1202 \quad 1258$

$\begin{array}{llll}9 & 255 & 1231 & 1284\end{array}$

$\begin{array}{lllllllll}15 & 263 & 407 & 407 & 410 & 410 & 456 & 465 & 492\end{array}$

$\begin{array}{lllllllll}494 & 500 & 503 & 504 & 507 & 510 & 511 & 520 & 528\end{array}$

$\begin{array}{lllllllll}529 & 532 & 532 & 686 & 689 & 690 & 1170 & 1200 & 1224\end{array}$

$\begin{array}{lllllllll}15 & 263 & 586 & 586 & 589 & 589 & 635 & 644 & 671\end{array}$

$\begin{array}{lllllllll}673 & 679 & 682 & 683 & 699 & 707 & 708 & 711 & 711\end{array}$

$1185 \quad 1195 \quad 1229$

$\begin{array}{lllllllll}15 & 263 & 531 & 532 & 710 & 711 & 1123 & 1126 & 1126\end{array}$

$\begin{array}{lllllllll}1127 & 1133 & 1135 & 1312 & 1316 & 1316 & 1317 & 1325 & 1329\end{array}$

$\begin{array}{lllllllll}1329 & 1330 & 1337 & 1341 & 1341 & 1342 & 1553 & 1555 & 1563\end{array}$

$\begin{array}{lll}1565 & 1572 & 1574\end{array}$ 